\title{
UMA COMBINAÇÃO MEC/MEF PARA ANÁLISE DE INTERAÇÃO SOLO-ESTRUTURA
}

NEWTON CARLOS PEREIRA FERRO

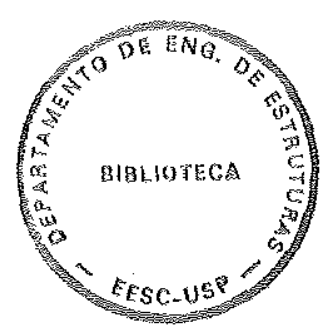

Tese apresentada à Escola de Engenharia de São Carlos, da Universidade de São Paulo, como parte dos requisitos para obtenção do título de Doutor em Engenharia Civil

ORIENTADOR: Prof. Tit. Wilson Sergio Venturini

São Carlos 


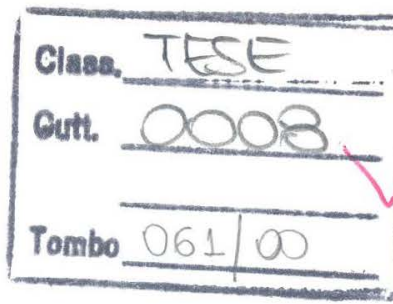

$51 / 51073133$

Ficha catalográfica preparada pela Seção de Tratamento da Informação do Serviço de Biblioteca - EESC/USP

Ferro, Newton Carlos Pereira

F395C Uma combinação MEC/MEF para análise de interação solo-estrutura / Newton Carlos Pereira Eerro. -- São Carlos, 1999.

Tese (Doutorado) -- Escola de Engenharia de São Carlos-Universidade de São Paulo, 1999.

Área: Engenharia de Estruturas.

Orientador: Prof. Dr. Wilson Sergio Venturini.

1. Método dos elementos de contorno. 2. Método dos elementos finitos. 3. Interação solo-estacas.

4. Interação solo-estrutura. 5. Combinação MEC-MEF . I. Título. 


\section{FOLHA DE APROVACÃO}

Candidato: Engenheiro NEWTON CARLOS PEREIRA FERRO

Tese defendida e aprovada em 14-91-1999

pela Comissão Julgadora:

Prof. Titular WILSON SERGIO VENTURINI (Orientador)

(Escola de Engenharia de São Carlos - Universidade de São Paulo)

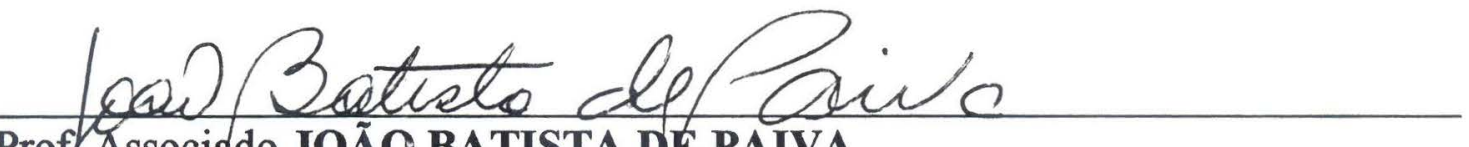

Prof Associado JOÃO BATISTA DE PAIVA

(Escola de Engenharia de São Carlos - Universidade de São Paulo)

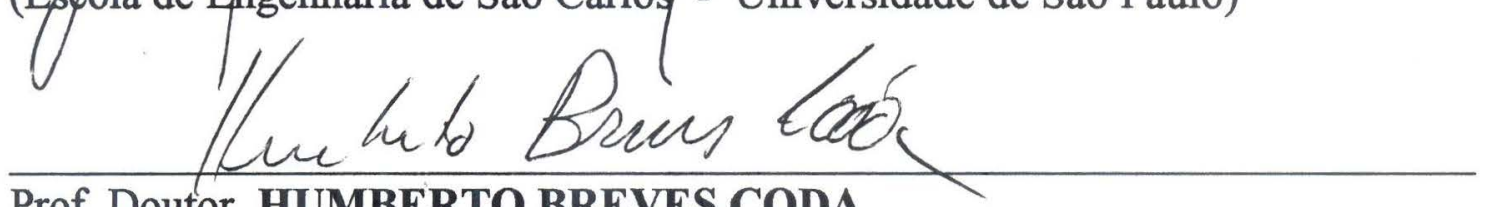

Prof. Doutor HUMBERTO BREVES CODA

(Escola de Engenharia de São Carlos - Universidade de São Paulo)

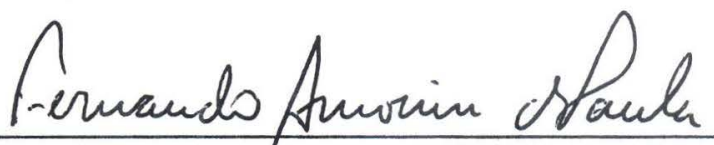

Prof. Adjunto FERNAYDo AMORIM DE PAULA

(Universidade Federal de Minas Gerais - UFMG)

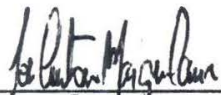

Prof. Doutor J JSÉ ANTONIO MARQUES CARRER

(COPPE - Universidade Federal do Rio de Janeiro)

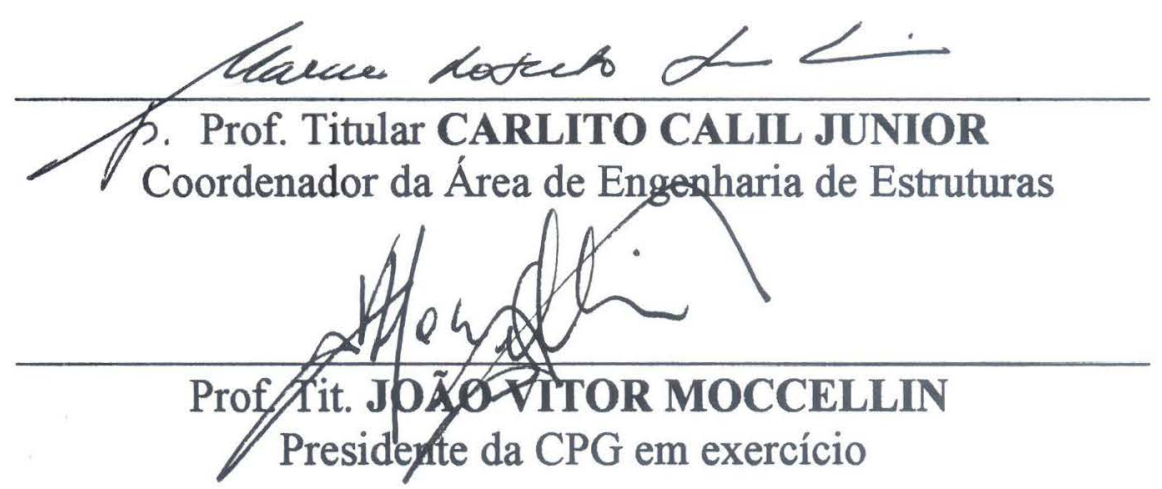


Aos meus pais Ivo e Maria e minha irmã Mirian.

A minha esposa Cassia e aos meus filhos Patrícia, Ricardo e Vinicius.

A meu sogro Petronio (in memoriam). 


\section{AGRADECIMENTOS}

Desejo expressar meus agradecimentos ao Prof. Tit. Wilson Sergio Venturini, por sua orientação e incentivo que permitiram a elaboração deste trabalho.

Sou especialmente grato ao Prof. Dr. José Carlos Angelo Cintra pelo seu empenho e dedicação que muito contribuíram para a conclusão do presente trabalho.

A Lauro Henrique de Mello Chueiri sou eternamente grato por sua amizade, incentivo e inestimável colaboração.

Aos professores Heitor Miranda Bottura e Ademar da Silva Lobo pelo apoio e especial colaboração.

A Vanilda Miziara Mello agradeço pela revisão dos textos.

Aos amigos, professores e funcionários do Departamento de Engenharia Civil da Unesp de Bauru meus agradecimentos pelo estímulo e apoio. Agradeço em especial a Edmilson Quirino Ramos pelos desenhos que ilustram este trabalho e às secretárias Marlene e Maria pelo carinho.

Aos professores e funcionários do Departamento de Estruturas da Escola de Engenharia de São Carlos, meus agradecimentos pela amizade demonstrada.

A todos que direta ou indiretamente colaboraram para que este trabalho se tornasse possivel.

A Coordenadoria de Aperfeiçoamento de Pessoal de Nível Superior - CAPES, pelo apoio financeiro. 


\section{SUMÁRIO}

LISTA DE FIGURAS

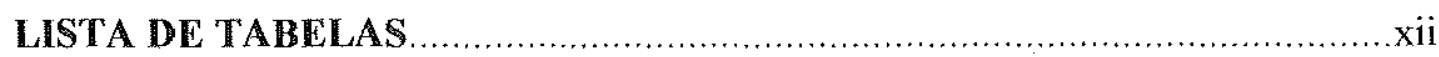

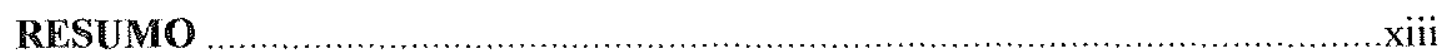

ABSTRACT

1 APRESENTAÇÃO

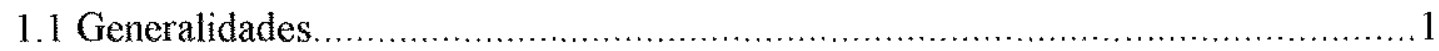

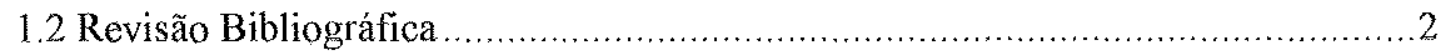

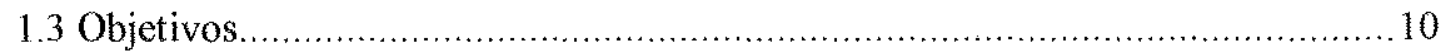

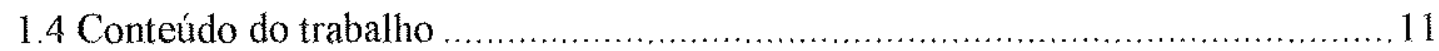

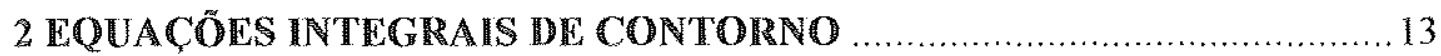

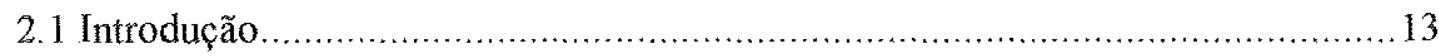

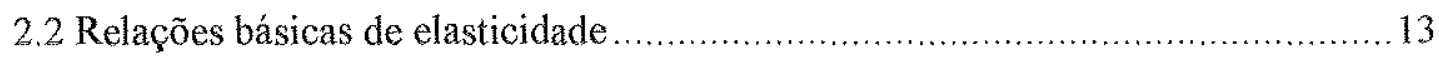

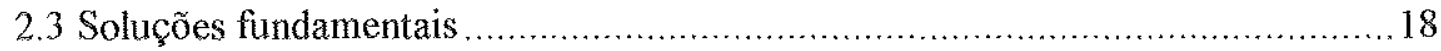

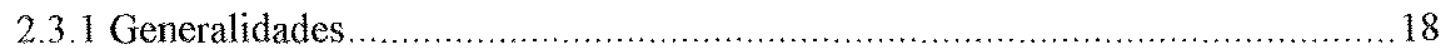

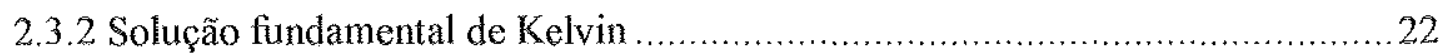

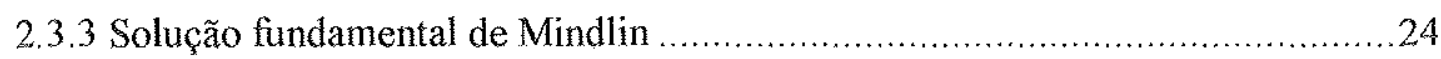

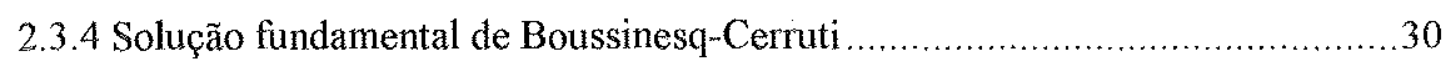

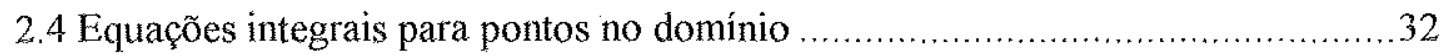

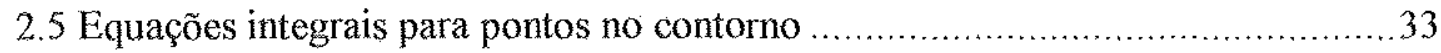

3 MÉTOdO DOS ElEMENTOS DE CONTORNO PARA

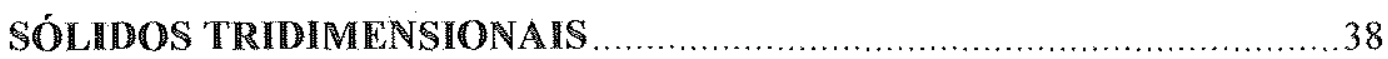

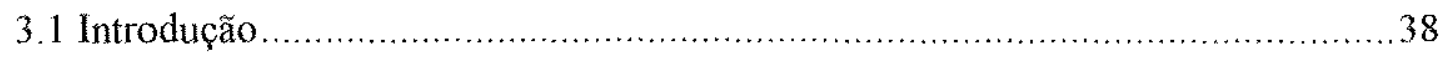

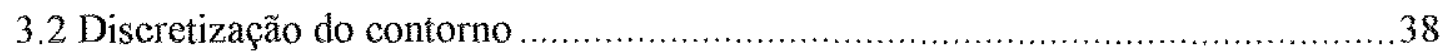

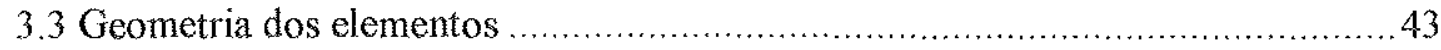

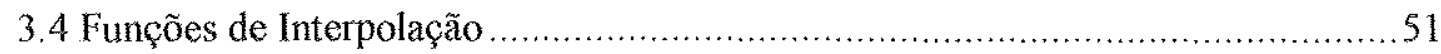




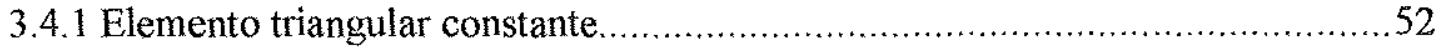

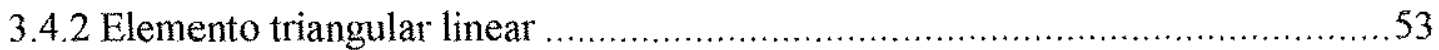

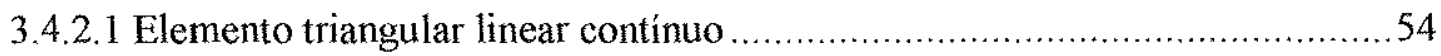

3.4.2.2 Elemento triangular linear descontínuo ........................................ 55

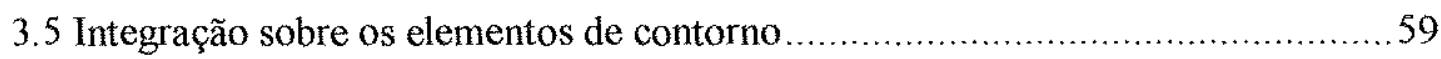

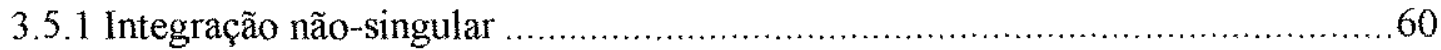

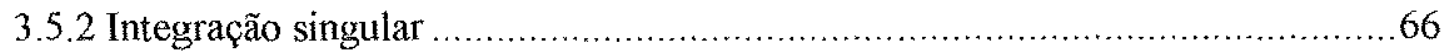

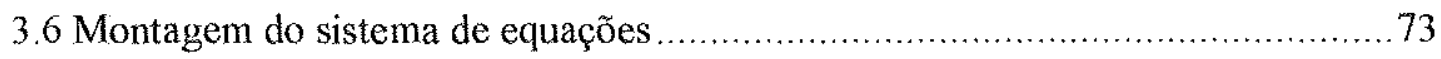

3.7 Deslocamentos em pontos do domínio .............................................. 79

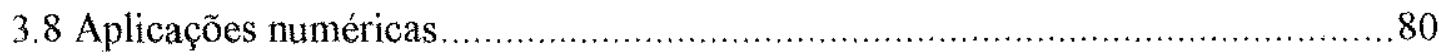

3.8.1 Exemplo 1 - área circular uniformemente carregada ................................ 80

3.8.2 Exemplo 2 - área retangular uniformemente carregada ............................ 84

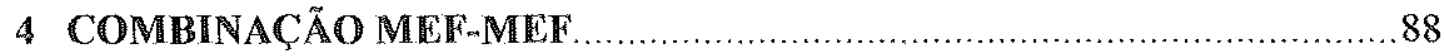

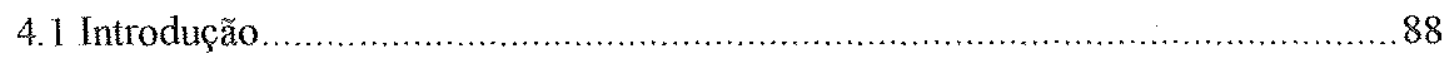

4.2 Interação de um meio tridimensional com estruturas de barras....................... 88

4.3 Discretização da equação integral de contorno …..................................... 90

4.4 A combinação do método dos elementos de contorno com o método dos elementos finitos ............................................................ 93

4.5 Rigidez do elemento fundação em estacas .......................................... 97

4.6 Translação da matriz de rigidez ....................................................... 101

4.7 Linhas de carga no domínio ..................................................... 105

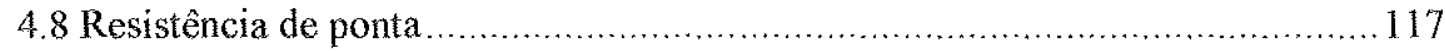

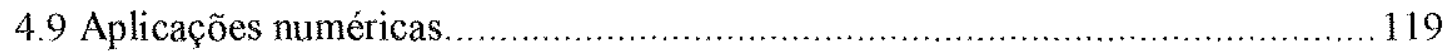

4.9.1 Estaca vertical sob carga vertical ............................................... 119

4.9.2 Interação entre duas estacas sob carga vertical ................................... 122

4.9.3 Estaca inclinada sob carga vertical.............................................. 125

4.9.4 Estaca inclinada sob carga horizontal.......................................... 127

5 MODELO NÃO LINEAR PARA O ACOPLAMENTO DO

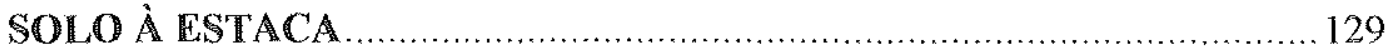

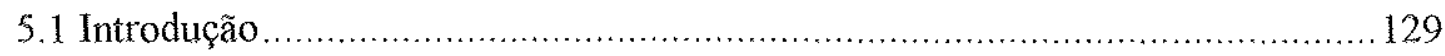

5.2 Princípios básicos da teoria da plasticidade ......................................... 129

5.3 Problemas elastoplásticos unidimensionais ........................................ 131 
5.4 Teoria da plasticidade no espaço tridimensional ................................... 135

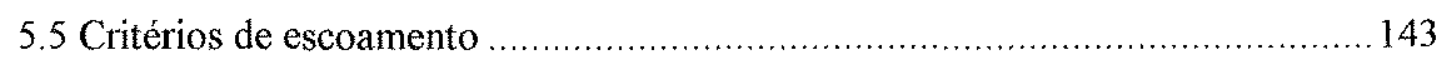

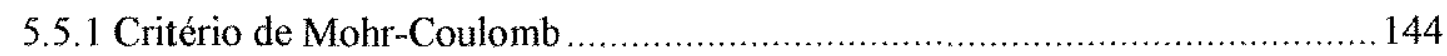

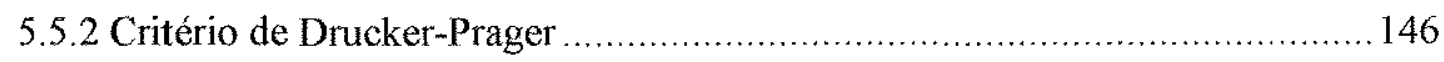

5.6 Formulação matricial das relações constitutivas ...................................... 148

5.7 Um modelo simples para a análise elastoplástica de solos na interface com estacas ................................................................... 150

5.8 Procedimento numérico para a solução do problema não-linear...................... 153

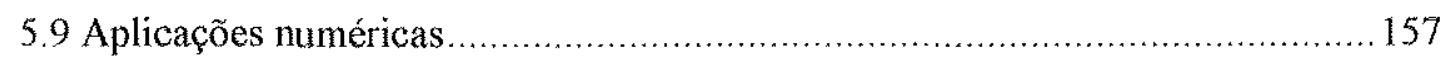

5.9.1 Exemplo 1 - Ensaio de Whitaker e Cooke ............................................ 157

5.9.2 Exemplo 2 - Estaca vertical com carga vertical ................................. 158

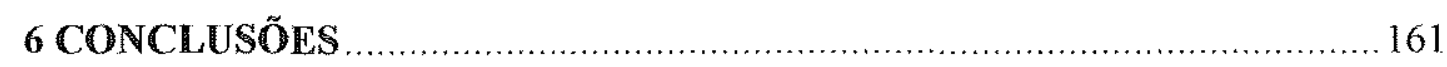

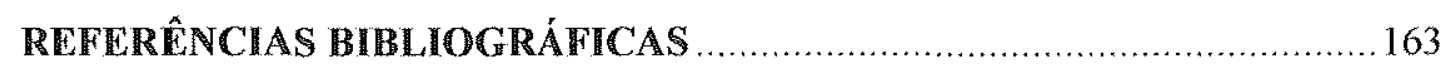




\section{LISTA DE FIGURAS}

FIGURA 2.1 - Corpo tridimensional de domínio $\Omega$ e contorno $\Gamma$

FIGURA 2.2 - Componentes das forças de superficie no tetraedro de

Cauchy

FIGURA 2.3 - Domínio $\Omega$ com o contorno definido pelas condiçôes

$$
\overline{\mathrm{u}}_{\mathrm{i}} \text { e } \overline{\mathrm{p}}_{\mathrm{i}} \text { em } \Gamma_{1} \text { e } \Gamma_{2}
$$

FIGURA 2.4. - Domínio $\Omega$ contido em $\Omega^{*}$

FIGURA 2.5 - Resposta em " $q$ " devido à uma força $F_{i}^{*}$ aplicada em

$$
\text { "S". }
$$

FIGURA 2.6. - Componentes do tensor de deslocamentos fundamentais

FIGURA 2.7 - Componentes do tensor de forças de superficie fundamentais

FIGURA 2.8 - Problema fundamental de Kelvin

FIGURA 2.9 - Problema fundamental de Mindlin 26

FIGURA 2.10 - Problema fundamental de Boussinesq-Cerruti

FIGURA 2.11 - Acréscimo ao domínio $\Omega$ de uma semi-esfera infinitesimal de domínio $\Omega_{\varepsilon}$

FIGURA 3.1 - Tipos de elementos utilizados para aproximar o contorno : a) triangulares; b) quadrilaterias

FIGURA 3.2 - Tipos de células para aproximar o domínio: a) tetraédricas; b) pentahédricas; c) hexaédricas

FIGURA 3.3 - Discretização do contorno de um corpo em elementos: a) constante; b) linear; c) quadrático. 41 
FIGURA 3.4 - Coordenadas globais e naturais (locais) para o elemento triangular plano...... 44

FIGURA 3.5 - Coordenadas cartesianas do ponto $\mathbf{P}$.............................................45

FIGURA 3.6 - Coordenadas naturais para alguns pontos do triângulo …................46

FIGURA 3.7 - Indicação dos sistemas de coordenadas ....................................... 50

FIGURA 3.8 - Elemento triangular constante .......................................... 52

FIGURA 3.9 - Elementos triangulares lineares: a) contínuo; b) e c) de transição; d) descontínuo

FIGURA 3.10 - Funções interpoladoras para o elemento triangular linear contínuo

FIGURA 3.11 - Posição e funções de interpolação dos pontos nodais do elemento triangular linear descontínuo 56

FIGURA 3.12 - Sistema de coordenadas homogêneas $\xi_{i}$ e $\bar{\xi}_{j}$, com $i=1,2,3$.

FIGURA 3.13 - Descontinuidades no contorno de um sólido tridimensional 58

FIGURA 3.14 - Coordenadas triangulares homogêneas do elemento e subelemento .62

FIGURA 3.15 - Divisão de um elemento em subelementos ................................64

FIGURA 3.16 - Elemento dividido em quatro subelementos ...........................65

FIGURA 3.17 . Sistema de coordenadas locais cartesiano e cilíndrico ...................67

FIGURA 3.18 - Esquema de integração em $\mathrm{r}$.............................................. 70

FIGURA 3.19 - Integração para ponto de colocação interno sobre os lados do elemento triangular ............................................ 72

FIGURA 3.20 - Elemento de colocação não nodal .............................................74

FIGURA 3.21 - Representação de nós múltiplos ............................................. 75

FIGURA 3.22 - Área circular uniformemente carregada ................................ 80

FIGURA 3.23 - Discretização da área circular carregada ................................... 81

FIGURA 3.24 - Deslocamentos verticais ao longo do eixo $x_{1} \ldots \ldots \ldots \ldots \ldots \ldots \ldots \ldots . . \ldots 3$

FIGURA 3.25 - Deslocamentos verticais ao longo do eixo $x_{3} \ldots \ldots \ldots \ldots \ldots \ldots \ldots \ldots . \ldots 3$

FIGURA 3.26 - Área retangular uniformemente carregada ............................... 84

FIGURA 3.27 - Discretização da área retangular carregada ................................ 85 


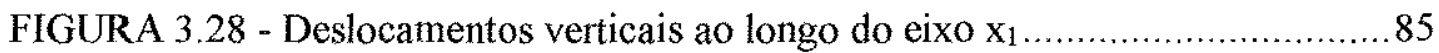

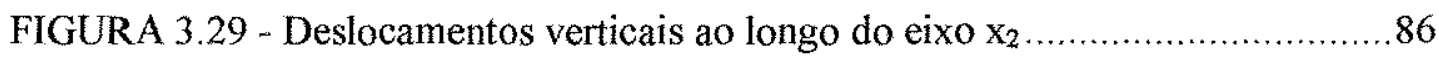

FIGURA 3.30 - Deslocamentos verticais ao longo do eixo $x_{3} \ldots \ldots \ldots \ldots \ldots \ldots \ldots \ldots . . \ldots 7$

FIGURA 3.31 - Deslocamentos verticais ao longo de um eixo paralelo ao eixo $\mathrm{x}_{3}$ que passa pelo vértice da área

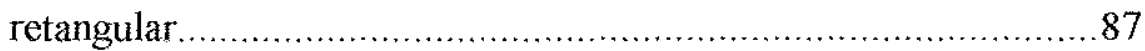

FIGURA 4.1 - Domínio tridimensional com linha de carga ...............................89

FIGURA 4.2 - Domínio discretizado com elementos finitos $\mathrm{e}$

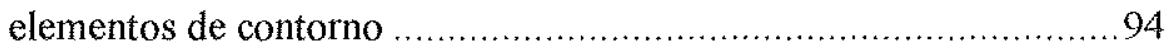

FIGURA 4.3 - Deslocamento unitário em relação ao eixo $x_{1} \mathrm{e}$

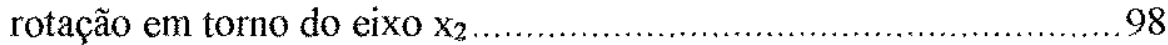

FIGURA 4.4 - Translação da rigidez do ponto $\overline{\mathrm{O}}$ para o ponto $\mathrm{O}$........................ 102

FIGURA 4.5 - Sistema de coordenadas locais para uma seção da estaca

FIGURA 4.6 - Elemento linear contínuo e as respectivas funções de interpolação

FIGURA 4.7 - Representação da distribuição das forças de interação em um elemento de estaca

FIGURA 4.8 - Valores nodais para deslocamentos e rotações nas direções $\mathrm{x}_{1}$ e $\mathrm{x}_{2}$.

FIGURA 4.9 - Representação do último elemento de uma estaca com indicação do nó adicional para considerar a resistência de ponta.

FIGURA 4.10 - Deslocamentos ao longo da estaca na direção $x_{3}$

FIGURA 4.11 - Forças de superfície ao longo da estaca na direção $\mathrm{x}_{3}$.

FIGURA 4.12 - Deslocamentos ao longo da estaca na direção $x_{3}$, considerando-se a resistência de ponta

FIGURA 4.13 - Forças de superficie ao longo da estaca na direção $\mathrm{x}_{3}$, considerando-se a resistência de ponta

FIGURA 4.14 -- Representação da interação de deslocamentos na direção $\mathrm{x}_{3}$ entre duas estacas verticais com cargas 
verticais para $\mathrm{k}=500$

FIGURA 4.15 - Representação da interação de deslocamentos na direção $\mathrm{x}_{3}$ entre duas estacas verticais com cargas verticais para $\kappa=100000$

FIGURA 4.16 - Deslocamentos na direção $\mathrm{x}_{1}$ para duas estacas sob carga vertical, $v=0$ e $\kappa=500$.

FIGURA 4.17 - Deslocamentos horizontais na direção $\mathrm{x}_{1}$ ao longo do comprimento de uma estaca inclinada

FIGURA 4.18 - Deslocamentos verticais na direção $\mathrm{x}_{3}$ ao longo comprimento de uma estaca inclinada

FIGURA 4.19 - Deslocamentos horizontais na direção $\mathrm{x}_{1}$ ao longo do comprimento de uma estaca inclinada, sob a ação de uma carga horizontal

FIGURA 4.20 - Forças de superficie na direção $x_{1}$ ao longo do comprimento de uma estaca inclinada, sob a ação de uma carga horizontal

FIGURA 5.1 - Diagrama tensão-deformação para um ensaio uniaxial 130

FIGURA 5.2 - Diagrama tensão-deformação para material elastoplástico perfeito.

FIGURA 5.3 - Diagrama tensão-deformação para um material elastoplástico com endurecimento

FIGURA 5.4 - Modelos de representação de comportamento com endurecimento.

FIGURA 5.5 - Representação do critério de Mohr-Coulomb

FIGURA 5.6-Representação geométrica das superfícies de escoamento de Mohr-Coulomb e Drucker-Prager no espaço das tensões principais 146

FIGURA 5.7 - Representação geométrica dos critérios de MohrCoulomb e Drucker-Prager no plano de tensões $\sigma_{1}-\sigma_{2}$

FIGURA 5.8 - Curva carga-deslocamento no topo da estaca 158

FIGURA 5.9 - Curva carga deslocamento no topo da estaca 


\section{LISTA DE TABELAS}

TABELA 3.1 - Valores correspondentes aos deslocamentos verticais

obtidos a partir da solução exata e numérica. 82 


\section{RESUMO}

FERRO, N. C. P. (1998). Uma combinação MEC/MEF para a análise de interação solo-estrutura. São Carlos, 1999. 193p. Tese (Doutorado - Escola de Engenharia de São Carlos, Universidade de São Paulo.

No presente trabalho, uma combinação do método dos elementos de contorno (MEC) com o método dos elementos finitos (MEF) é apresentada para a análise da interação entre estacas e o solo, considerado como um meio infinito tridimensional e homogêneo. $\mathrm{O}$ meio contínuo tridimensional de domínio infinito é modelado pelo $\mathrm{MEC}$, enquanto as estacas consideradas como elementos reticulares são tratadas pelo MEF. As equações das estacas oriundas do método dos elementos finitos são combinadas com as do meio contínuo obtidas a partir do método dos elementos de contorno, resultando em um sistema completo de equações, que convenientemente tratadas, proporcionam a formulação de coeficientes de rigidez do conjunto soloestacas. Finalmente, uma formulação para a análise do comportamento não-linear do solo na interface com a estaca é desenvolvida, tornando o modelo mais abrangente.

Palavras-chave: método dos elementos de contorno; método dos elementos finitos; interação solo-estaca, interação solo-estrutura; combinação MEC-MEF; plasticidade. 


\begin{abstract}
FERRO, N. C. P. (1998). A BEM-FEM combination for soil-structure interaction analysis. São Carlos, 1998, 226p. Tese (Doutorado - Escola de Engenharia de São Carlos, Universidade de São Paulo.
\end{abstract}

In the present work a combination of the Boundary Element Method (BEM) and the Finite Element Method (FEM) is used for pile-soil interaction analyses, considering the soil as a homogeneous, three-dimensional and infinite medium. The three-dimensional infinite continuous medium is modeled by the BEM, and the piles are, considered as beam elements, modeled by the FEM. This combination also is used for studying the interaction of plates sitting on a continuous medium. The pile equations generated from the FEM are combined with the medium equations generated from the BEM, resulting a complete equation system. Manipulating properly this equation system, a set of stiffness coefficients for the system soil-pile is obtained. Finally, to make the model more comprehensive, it presented a formulation to take into account the soil nonlinear behavior at the pile interface.

Keywords: boundary element methods; finite element methods; pile-soil interaction; structure-soil interaction, BEM-FEM combination; plasticity. 


\section{APRESENTAÇÃO}

\subsection{GENERALIDADES}

O uso combinado do Método dos Elementos de Contorno (MEC) com o Método dos Elementos Finitos (MEF) tem-se mostrado adequado para a resolução de um grande número de problemas de Engenharia. Pode-se citar, por exemplo, aqueles envolvendo domínios inlinitos, interagindo com clementos estruturais de dimensôes linitas, demonstrando ser este um dos melhores campos onde as combinações são recomendadas.

A principal vantagem da combinação $\mathrm{MEC}-\mathrm{MEF} \dot{\mathrm{c}}$ a possibilidado de se empregar o método mais apropriado para cada uma das subestruturas. Uma desvantagem a se destacar são as diliculdades para o tratamento dos sistemas de equaçōes cnvolvidos, visto que os do MEF resultam em matrizes simétricas $\mathrm{c}$ os do $\mathrm{MEC}$, em matrizes cheias c nào simétricas.

Enumeraram-se algumas das vantagens e desvantagens da combinação $\mathrm{MEC}$ MEF para o tratamento do problema da interação supcrestrutura-solo-fundação.

Sabe-se que a maioria dos programas utilizados em cálculos estruturais tratam o comportamento da estrutura considerando-a como parte isolada, independente, portanto, do comportamento da fundação. Nas estruturas usuais, tal aproximação não deve apresentar problemas sćrios na avaliação final dos esforços. Entrctanto, uma consideração mais realista dos esforços e deformações dos elementos que compōem tanto a superestrutura quanto a fundação é sempre desejável.

Fste objetivo foi aleançado, inicialmente, dentro das propostas formuladas, no 
trabalho de mestrado.

O que se pretende, com este trabalho, é o desenvolvimento de uma formulação mais abrangente, lunção das novas consideraçôes a serem implenentadas no modelo proposto, de forma a se poder avaliar com mais realismo a interação fundaçãosuperestrutura.

\subsection{REVISÃO BIBLIOGRÁFICA}

O Método dos Elementos de Contorno teve desenvolvimento expressivo mais recentemente, incorporando as caracteristicas de método numérico. No cntanto, as equaçòes integrais já são conhecidas há bastante tempo. Segundo ELLIOT (1980), foi ABEL (1881) quem primeiro deduziu uma cquação integral para a solução de um problema lisico, a do pêndulo isócrono. LIOUVILLE (1837) transformou um problema de valor inicial $\mathrm{em}$ uma equaçào integral e a resolveu usando aproximações sucessivas. Um novo avanço se deu com a resoluçào do problema da teoria do potencial; VOLTERRA (1956), em 1884, estudou a distribuição de cargas elétricas na superficic de uma esfera utilizando equaçồs integrais. Deveu-se, no entanto, a FREDHOLM (1903), a primeira investigação rigorosa das equações integrais. Foi cle quem primeiro apresentou um estudo das aplicações das equaçoes integrais lineares à solução de problemas de valor de contorno em clastostática.

Posteriormente, o método foi aplicado para a solução de problemas de elasticidade, notadamente pelos pesquisadores sovicticos. Os trabalhos de MUSKHELISHVILI (1953), MIKHLIN (1957, 1965 a e 1965b) \& KUPRADZE (1965) são bem conhecidos.

O método de solução de MUSKHELISHVILI (1953) bascia-se na tcoria das variáveis complexas, não podendo ser estendido aos problemas de elasticidade tridimensional. Os trabalhos de MIKHLIN $(1957,1965)$ sobre as equações integrais singulares multidimensionais s̃̃o muito mais úteis neste aspecto.

KUPRADZE (1964) descreveu uma formulação integral para destocamentos de corpos em regime elástico. MASSONET (1965) apresentou uma solução numérica para problemas de valores de tensões no contorno, formulada em temos de uma equação 
integral singular, vetorial de $2^{\text {a }}$ espécic. Sua fomulação bascou-se no uso da solução de KELVIN $^{*}$ apud LOVE (1944). WATSON $(1968,1972,1973)$ e VAN BUREN (1968) utilizaram a formulação de Kupradze para problemas clastostáticos, de acordo com BANERJEE (1976).

Todas as Cormulaçōes até aqui apresentadas são denominadas de métodos indiretos, já que a soluçào do problema era sempre obtida $\mathrm{cm}$ temos de fontes lietícias aplicadas ao contorno, e que, após sua determinação, permitiam o cálculo das variávcis fisicas do problema.

JASWON (1963) c SYMM (1963) introduziram um equacionamento do problema clástico bidimensional empregando variaveis reais c ainda mantendo uma função de tensão auxiliar, sendo, por isso, chamado de método scmi-direto, usado na resolução de problemas de potencial.

Os diversos trabalhos até aqui citados contribuíram para o desenvolvimento e entendimento das equações integrais, mas nenhum deles apresentava as características necessárias de modo a poder interpretá-los como um método numérico. O trabalho de RIZZO (1967) para a solução de problemas de elasticidade bidimensional foi o primeiro em que o tratamento dado às equações integrais toma a forma de técnica numérica similar a dos demais métodos. Foi também o primciro a propor a formulação direta na utilização das equações integrais, relacionando valores não lictícios no contorno, isto é, deslocamentos e forças de superfícic. CRUSE $(1969,1973)$ logo estendeu a formulação à elasticidade tridimensional. Em seus trabalhos, Rizzo e Cruse, partindo da identidade de SOMIGLIANA (1886), escrita para a determinação de deslocamentos no interior de um corpo, e cmpregando o tcorema de BETTI (1872), obtiveram uma cquação integral da forma da identidade de Somigliana, agora, no entanto, para calcular deslocamentos também em pontos situados no contomo. As equações integrais foram discretizadas dividindo-se o contorno en um número de clementos planos, assumindo-se como incógnitas as forças de superlicic e deslocamentos considerados constantes sobre o elemento.

CRUSE \& VANBUREN (1971) foram os primciros a aplicarem a formulação a

\footnotetext{
* KELVIN, L. (1863) Phil. Trans. Roy. Soc. v. 153 apud LOVE. A.E.H. (1944) A treatise on the mathematical theory of elasticity. New York, Dover Publications.
} 
um sólido tridimensional não-linear considerando a influência de uma fratura.

Neste mesmo ano, SWEDLOW \& CRUSE (1971) apresentaram uma fomulação para simular matcriais clastoplásticos, anisotrópicos e compressíveis, considerando, ainda, o encruamento na relação tensão-deformação. Dando continuidade a este trabalho, RICARDELLA (1972) considerou o critério de VON MISES (1913), com encruamento para a modulação de cfeito plástico, na análise tridimensional de tensōes. CRUSE (1974) propôs a utilização de elementos no contono, com forças de superficic e deslocamentos com variação linear sobre estes clementos. As incógnitas estão, agora, definidas nas extremidades ou "nós" dos elementos. O sistema de equações lineares foi, cntão, obtido, escrevendo-se as equações integrais nesses pontos.

LACHAT (1975), estudando problemas clásticos bi e tridimensionais, conseguiu dar uma generalização bem maior ao método, introduzindo representações paramétricas dos elementos de superficic (clementos curvos com variação quadrática) c das funções de aproximação das forças de superlicie e deslocamentos, consideradas com variações lincarcs, quadráticas c cúbicas. As integrais são calculadas numcricamente, utilizando as fómulas da quadratura de Causs. A lécnica de sub-regiões é utilizada não só para modclar corpos não homogêncos, mas como um recurso para facilitar a resolução do sistema linal de equações, que passa, assim, a ser delinido por blocos.

Deve-se a BREBBIA (1978a), a formulação das cquaçōes integrais a partir do método dos resíduos ponderados. Combinaçôes entre o método das equaçòes integrais $\mathrm{c}$ outras técnicas numéricas, podiam, agora, ser equacionadas de mancira consistente, utilizando-se as respectivas formulações $\mathrm{cm}$ termos de resíduos ponderados. Foi BREBBIA (1978b) quem denominou a técnica de "MÉTODO DOS ELEMENTOS DE CONTORNO" - MEC - em substituição a "Método das Equações Integrais de Contorno" - BIEM -, até então utilizado.

A partir de 1978, várias Conferencias e trabathos publicados (dentre eles, destacam-se os de BREBBIA (1978c, 1980, 1981 c 1982)), tornaram o Método dos Elementos de Contorno mais conhecido, abrangendo as mais variadas áreas de engenharia, podendo-se citar as relativas a: não-linearidade lisica e geométrica; plasticidade; viscoclasticidade; viscoplasticidade; no-tension; mecânica da fratura; mecânica dos solos c das rochas; contato; percolação; vibrações; propagação de ondas; placas; cascas; concentação de tensōes: propagação de tensões; interação solo- 
estrutura; combinações com outros métodos para análise de problemas diversos, dentre outros.

Há que se salientar os avanços alcançados na formulação de não-linearidade lisica c geomćtrica, a partir dos trabalhos de: BUI (1978), TELLES \& BREBBIA (1979, 1980a c 1980b), MUKERJEE (1982), NOVATI \& BREBBIA (1982), MUKERJEE \& CHANDRA (1984), MUKERJEE \& RAJIYAH (987), CATHIE \& BANERJEE (1982), BANERJEE \& DAVIS (1984) BANERJEE cl al. (1985), MUSTOE (1984), BRUNET (1982), NOVATTI \& BREBBIA (1982), MAIER \& NOVATI (1983), MAIER et al. (1984) e GIODA ot al. (1984).

Em mecânica dos fluidos, são de grande importância os trabalhos de: HESS \& SMITH (1967), HESS (1975), BÉZINE \& BONNEAU (1981), YANO \& KIEDA (1980) c WU (1980, 1982, 1985 e 1987), EL REFAEE el al. (1982).

Na formulação do método dos clementos de contorno para placas destacam-se os trabathos de: TOTTENHAM (1979), BÉZINE (1978, 1980), BÉZINE cl al. (1985), TANAKA (1984), KAMIYA \& SAWAKY (1982), COSTA \& BREBBIA (1985) c MORJARIA \& MUKHERIEE (1980), dentre outros. Na análise de tensões no meio contínuo, com aplicações em meios plásticos c viscoplásticos, são cxcmplos os trabalhos de BRADY \& BRAY (1978), BRADY (1979), VENTURINI (1982) e VENTURINI \& BREBBIA (1984).

Nas aplicações do método a problemas envolvendo ações dinâmicas, é necessário destacar-se os trabalhos de: MANOLIS \& BESKOS (1981), DOMINGUEZ \& ALARCON (1981), MANSUR (1983) e WOLF (1985).

Após um breve histórico da evolução do método dos elementos de contorno, cnumerar-se-ão os trabahos mais relevantes, propondo formulaçôes para a análise de problemas de fundaçôes em estacas $\mathrm{cm}$ meio semi-infinitos e aqueles que, devido às suas características, exigen a combinação entre partes estruturais diferentes, tratadas por métodos numéricos também diferentes.

A utilização primeira de equações integrais na deteminação de deslocamentos cm estacas é bastante antiga c deveu-se a POULOS \& DAVIS (1968).

O trabalho considera uma única cstaca cilíndrica incompressivel, submetida à ação de uma carga axial, $\mathrm{cm}$ um meio semi-infinito, isótropo e homogêneo, admitindo- 
se não existirem deslocamentos relativos entre o solo e a estaca. A estaca foi discretizada cm clementos cilíndricos, submetidos em seu contorno a uma tensão de cisalhamento uniforme ao longo de cada um dos elementos, e uma base alargada submetida apenas à tensão axial.

Foi empregada a soluçâo fundamental de MINDLIN (1936), considcrando-se apenas os deslocamentos verticais. As integrações ao longo do comprimento da estaca foram efetuadas analiticamente e no sentido circunferencial de 0 a $2 \pi$, numericamente (em intervalos de $\pi / 50$ ).

No mesmo ano, POULOS (1968) apresentou um trabalho para a análise de grupos de cstacas, a partir da formulação anterior. A análise considera a interação entre duas estacas identicas, igualmente carregadas c dispostas verticalmente, $\mathrm{c}$ os incrementos de deslocamentos verticais em cada estaca, devido à interação, são expressos $\mathrm{cm}$ termos de um coeficiente $\alpha$. Estende-se o cálculo para grupos de estacas, simétricos, com mesmas propricdades físicas c igualmente carregadas.

Um aprimoramento na formulação surgiu com o trabalho publicado por MATTES \& POULOS (1969), passando a considerar a compressibilidade da estaca no cálculo dos deslocamentos vericais. Estes são expressos em termos de uma cquação diferencial e escritos na forma de diferenças finitas. Impondo-se a compatibilidade de deslocamentos na interface solo-estaca, determinam-se as tensòes de cisalhamento ao longo da estaca c, posteriomente, os deslocamentos.

POULOS (1971a) apresentou uma formulação similar à anterior para uma cstaca vertical submetida a força horizontal c momento, estendeu-a logo a seguir para grupos de cstacas dispostas simetricamente (POULOS, 1971b). BUTTERFIELD \& BANERJEE (1971a) retomaram a análise clástica para uma estaca ou grupo de estacas, admitidas compressíveis e submetidas a carga axial, agora com a utilização do método indireto das equações integrais. Neste trabaho, consideram-se os deslocamentos horizontais, advindos da carga vertical. No mesmo ano, BUTTERFILD \& BANERJEE (1971b) apresentaram um trabalho para grupo de estacas, considerando-se a interaçào solo-cstaca-bloco de capcamento.

Uma Cormulação mais geral para a análise de grupo de estacas inclinadas foi aprescntada por BANERJEE \& DRISCOLL (1976), utilizando o método indircto das 
equações integrais. $\mathrm{O}$ grupo de estacas sob um bloco rígido pode estar submetido à ação de carga vertical, horizontal e momento, mantendo-se a simetria do conjunto de forma a reduzir as dimensões das matrizes envolvidas. As tensões nas direções horizontal e vertical são admitidas uniformes ao longo de cada elemento que compõe a estaca. São aplicados ao bloco deslocamentos unitários nas direções vertical c horizontal c rotação unitária, rotação esta transformada em deslocamentos. Estes são transferidos às cabeças das estacas, os quais, levados ao sistema de cquações, após resolvido, produzem uma matriz de rigide $z$ do conjunto de estacas de ordem 3.

Uma extensão ao trabalho anterior foi proposta por BANERJEE (1978), onde o módulo de deformação do solo é considerado com variação linear ao longo do comprimento da cstaca.

Com relação ao desenvolvimento de formulações aplicadas a sólidos elásticos tridimensionais, o trabalho de NAKAGUMA (1979) apresenta uma formulação direta do método dos elementos de contorno, baseado nas soluções fundamentais de Kelvin, Mindlin e Boussinesq-Cerruli. A utilização das soluçoes de Mindlin e BoussinesqCerruli no estudo de sólido de dominio semi-intinito, com aplicações em interação soloestruturas e cseavaçōes, introduziu grande versatilidade ao método, visto que dispensa a discretização da superfície do meio por hipótese livre de forças de superlicic.

A utilização do método dos clementos de contorno na análise de problemas de engenharia ficaria restrita se o método não permitisse a combinação entre partes de diferentes naturezas, tendo também, muitas vezes, que serem tratados por métodos numéricos diferentes. Algoritmos numéricos que combinam o MEC com outras técnicas já foram propostos por diversos autores.

A idéia da combinação do MEC com o MEF parece ter sido utilizada, inicialmente, por McDONALD \& WEXLER (1972), para a análise de problemas na área de engenharia elétrica, c por CHEN \& MEI (1974), em mecânica dos fluidos.

Os trabalhos de ZIENKIEWICZ et al. (1977), de SHAW \& FALBY (1977) c de OSIAS et al. (1977), foram os primeiros a tratar sólidos onde uma parte é analisada via elementos de contono e o restante do domínio é discretizado e analisado pelo método dos elementos linitos.

Dois dos primeiros trabalhos que estudam a interação solo-estrutura, c que 
consideram a combinação de dois métodos numéricos, foram o de MATTES \& POULOS (1969), para a análise do comportamento de uma estaca, e o de BUTTERFIELD \& BANERJEE (1971a,1971b), que estendeu a análise para grupos de cstacas. Nestes trabalhos, o solo é tratado pelo método dos elementos de contomo e as estacas, pelo método das diferenças finitas.

Outros trabalhos seguiram-se, podendo destacar-se os de KELLY et al. (1979), BREBBIA \& GEORGIOU (1979) c BEER \& MEEK (1981), que tratam da combinação do MEF com o MEC, sendo que os dois últimos se detêm na análise de domínios inlinitos, com aplicaçôes a problemas de Geomecânica.

É importante destacar, ainda, os trabalhos de VALLABHAN et al. (1984), VALLABHAN \& SIVAKUMAR (1986), BEER (1986), PAULA et al. (1987), VALLABHAN (1987), MESSAFER \& COATES (1989), dentre outros, todos voltados à análise da interação solo-estrutura en domínios bi e tridimensionais, com aplicaçǒes em Geomecânica. MEEK (1988) apresentou um sistema computacional envolvendo a combinação MEF - MEC, voltado à análise de problemas clásticos tridimensionais.

Ainda em relação à implementação da combinação entre estes métodos com aplicação a problemas de geomecânica c de interação solo-estrutura, podemos citar, dentre outros, os trabalhos de BRADY \& WASSYNG (1981), BEER (1983), OHKAMI \& KUSAMA (1985), CHEN \& QIAN (1986), BEER \& SWOBODA (1988), BEER (1989), VON ESTORFF \& BAUSEL (1989), LU et al. (1991), BELYTSCHKO \& LU (1991), EBERHARDSTEINER ol al. (1993), FLOREN \& DUDDECK (1993), PAIVA \& BUTTERFIELD (1994).

A aplicação do método dos elementos de contorno $\mathrm{cm}$ problemas de geomecânica, para a análisc de estados de tensões e deformaçooes, não linearidades físicas, materiais não homogêncos no domínio $\mathrm{cm}$ análise, exigindo o uso da técnica das sub-regiões, descontinuidade encontrada $\mathrm{cm}$ matcriais rochosos, meios clásticos, clastoplásticos e clasto-viscoplásticos, são alguns dos temas que têm despertado o interesse de grande número de pesquisadores, podendo destacar-se: BANERJEE \& BUTTERFIELD (1976), VENTURINI (1982,1983 e 1984), ALARCON ct al. (1983), BESKOS (1988), MAIER \& NOVATI (1987 c 1988). MANOLIS et al. (1993).

Inúmcros são os trabathos envolvendo a análise de problemas não lineares com o emprego do método dos clementos finitos, podendo-se citar dentre tantos os dos 
seguintes autores: ZIENKIEWICZ et at. (1969), ZIENKIEWICZ (1971), NAYAK \& ZIENKIEWICZ (1972), OWEN \& HINTON (1986), COOK el al. (1989), PROENC.A (1989), que foram tomados como básicos para o desenvolvimento do assunto neste trabalho.

As aplicações mencionadas do $\mathrm{MEC} \mathrm{cm}$ geomecânica podem, ainda, ser estendidas para se considerar as estruturas de fundações diretas ou enterradas no meio contínuo, caso freqüente na análise de obras civis. Além dos autores já nomeados, outros trabalhos também relevantes têm sido apresentados, por autores como: POULOS (1982, 1989), CHOW (1986), LEUNG \& CHOW (1985), MADHAV \& BUDKOWSKA (1986), MITA \& LUCO (1986), WOLF \& DARBRE (1986), BUDHU \& DAVIES (1988), CHIN \& CHOW (1990), CHIN et al. (1990), RAMALHO (1990), RAMALHO \& VENTURINI (1990), BUTTERFIELD \& PAIVA (1993), KAYNIA (1993), LEE (1993), PAK \& JI (1993), contribuindo na formulação de diversos procedimentos para interação solo-estacas.

Nas estruturas de lundações, a diversidade de matcriais que constituem o domínio em análise exige o uso da técnica das sub-regiões para tratar as diferentes partes delinidas pelo mesmo tipo estrutural. Diversos algoritmos numéricos foram propostos para a resolução de sistemas de equações, para tais siluações. CROTTY (1982) propôs um algoritmo bascado na climinação de blocos de zeros da matriz principal. Outros trabalhos tem sido publicados, apresentando algoritmos que reduzem o tempo de resolução dos sistemas ou cmpregam oulras formas de tratámos, tal cono o procedimento de condensação estática. Dentre cstes, podemos citar: SHARMA ef al (1985), DIVAKAR \& FAFITIS (1989), KANE et al. (1990), CAl ct al. (1991), REZAYAT (1992), KANE \& GURU (1993), MAIER a al. (1993a).

É importante mencionar os trabalhos que vêm sendo desenvolvidos na EESCUSP, particularmente aqueles envolvendo a utilização do método dos elementos de contomo e sua combinação com o método dos elementos finitos en aplicações em meios infinitos e scmi-infinitos.

CODA (1993) apresentou uma formulação tridimensional dinâmica transiente para a análise da interação solo-estrutura, em que a cstrutura é modelada pelo MEF c o meio contínuo, admitido clástico-linear, é modelado pelo MEC. O acoplamento da estrutura com o meio contínuo é realizado considerando-se a técnica das sub-regiões e 
sapatas rígidas são admitidas como elementos de ligaçào.

KOMATSU (1995) estudou uma combinação MEC-MEF para cstruturas reticuladas em domínio bidimensional. Os elementos de barras são tratados pelo MEF, cnquanto o meio contínuo, que pode ser homogêneo ou não homogêneo, é modelado pelo $\mathrm{MEC}$.

BARRETO (1995) propõe uma análise de flexão de placas sobre base clástica , utilizandomse apenas das fomulações do MEC, ou scja, tanto os problemas tridimensionais quanto os problemas de placas são tratados pela formulação de contorno para casos clastostáticos c clastodinâmicos.

\subsection{OBJETIVOS}

Os objctivos deste trabalho estão relacionados a implementações en uma formulação desenvolvida por FERRO \& VENTURINI (1991 c 1992) c FERRO (1993), aplicando-se a combinação MEC - MEF para a análise da interação solo-cstacas. Nela, as estacas são consideradas como clementos de barras verticais c modeladas pelo método dos elementos finitos c o solo, cono um domínio inlïnito, tridimensional, homogêneo, clástico-linear é tratado pelo método dos clementos de contorno. Com isto, obtém-se um sistema de equações que, convenientemente tratado, resulta $\mathrm{em}$ uma matriz de rigidez do conjunto solo-estacas.

Dentro desse contexto, pretende-se efetuar os seguintes desenvolvimentos à Cormulaçào existente:

1. Gencralização do modelo de estacas empregado, podendo, assim, considerarse estacas inclinadas, isto $\dot{c}$, disposição geral dos clementos lineares, imersos no meio contínuo.

2. Consideraçào de subelenentos ou integraçoes analíticas, quando os pontos de carga e de campo estiverem definidos no mesmo clemento que está sendo integrado, objetivando minimizar erros, visto que, devido ao pequeno diâmctro das estacas, cstas integrais são quase-singulares. 
3. Pretende-se desenvolver procedimentos de outras fundações e outras formas de carregamento que possam influenciar na fundação em estudo.

4. Aplicação de novos procedimentos de integração numérica objetivando minimizar possiveis crros cnvolvidos.

5. Finalmente, o desenvolvimento de uma formulação para análise do comportamento não linear do solo na interfacc com a estaca.

Após a formulação matemática de cada um dos itens mencionados, estes serão implementados $\mathrm{em}$ algoritmos numéricos, desenvolvendo-se, assim, um programa $\mathrm{cm}$ linguagem FORTRAN para microcomputador.

\subsection{CONTEÚDO DO TRABALHO}

Com base nos objetivos enumerados no item anterior, no Capitulo 2 são apresentadas as relações da teoria da clasticidade linear para sólidos tridimensionais, as equações integrais de contorno e as soluções fundamentais de Kelvin, Mndlin e Boussinesq-Cerruti.

No Capítulo 3, o contomo do sólido tridimensional é discretizado $\mathrm{cm}$ clementos cuja geometria é aproximada em função de scus nós geométricos por meio de lunções de interpolação. Sobre esses clementos são também aproximados os deslocamentos c forças de superfícic, em funçào de scus valores nodais. Aplicandose as equações integrais em número igual ao das incógnitas, são transformadas $\mathrm{em}$ um sistema de equações algébricas lineares que, após impostas as condições de contomo, pode ser resolvido, fornecendo as incógnitas no contorno, com as quais podem ser determinados os esforços e deslocamentos em pontos do domínio. Finalmente, são apresentadas algumas aplicaçǒes numéricas, mostrando a validade da Cormulação adotada.

No Capítulo 4, apresenta-se a inclusão do termo adicional nas equações integrais de contorno devido às linhas de cargas correspondentes às estacas, Cormuladas de maneira geral. A discretização do contorno, a modelagem das estacas, os procedimentos para a combinação $\mathrm{MEC}$ - MEF, tanto para elementos reticulares como para clementos triangulares planos, são desenvolvidos. Após a combinação dos 
métodos, uma formulação para a obtenção da matriz de rigidez da fundação é apresentada. A precisão das integrais ć cuidadosamente estudada, mostrando-se a técnica dos subclementos, que é então abordada.

O Capítulo 5 apresenta uma formulação para a análise não linear do solo na interface com as estacas. Alguns conceitos e hipóteses da teoria da plasticidade são abordados, bem como sua aplicação ao problema ora $\mathrm{em}$ estudo. Para a solução do sistema não-linear, admitiu-se um algoritmo incremental $\mathrm{e}$ iterativo, sem que haja a necessidade de atualização das matrizes envolvidas. São apresentados no final do Capítulo, alguns exemplos numéricos.

As considerações finais e as conclusões do trabalho são apresentadas no Capítulo 6. 


\section{EQUAÇÕES INTEGRAIS DE CONTORNO}

\subsection{INTRODUÇÃO}

Neste capítulo serào apresentadas as equações para análise clástica linear de sólidos tridimensionais, empregando-se notação indicial, bem como as equaçōes integrais básicas para a utilização do método dos elementos de contorno.

Dedica-se um item à apresentação das soluções fundamentais para o espaço infinito (Kelvin) e semi-infinito (Mindlin e Boussinesq-Cerruti), discutindo-se as relaçòes existentes entre clas.

Finalmente, serão apresentadas as equações integrais para pontos internos $\mathrm{e}$ externos ao domínio, assim como para os pertencentes ao contorno. O objetivo é escrever uma expressão que relacione deslocamentos $\mathrm{c}$ forças de superfǐcie $\mathrm{cm}$ pontos do contorno. Esta equação, posteriormente, será aproximadamentc numericamente resultando em um sistema de equações algébricas, o que será abordado no capítulo 3.

\subsection{RELAÇÕES BÁSICAS DE ELASTICIDADE}

Scja um corpo $\Omega+\Gamma$, cm que $\Omega$ é uma região tridimensional aberta e $\Gamma$ seu contorno, conforme apresentado na Fig. 2.1. Assume-se que o material constituinte desse corpo seja clástico linear, homogêneo e isótropo, para o qual são definidas duas constantes independentes $\mathbf{E} \subset v$, respectivamente, módulo de deformaçào e coeficiente de Poisson. A partir destas, podem ser definidos o módulo 
de deformação transversal $\mathbf{G}$ e a constante de Lamé $\lambda$. As relações entre tais constantes podem ser escritas em função das duas primeiras como:

$$
\begin{aligned}
& G=\frac{E}{2(1+v)} \\
& \lambda=\frac{v E}{(1+v)(1-2 v)}
\end{aligned}
$$
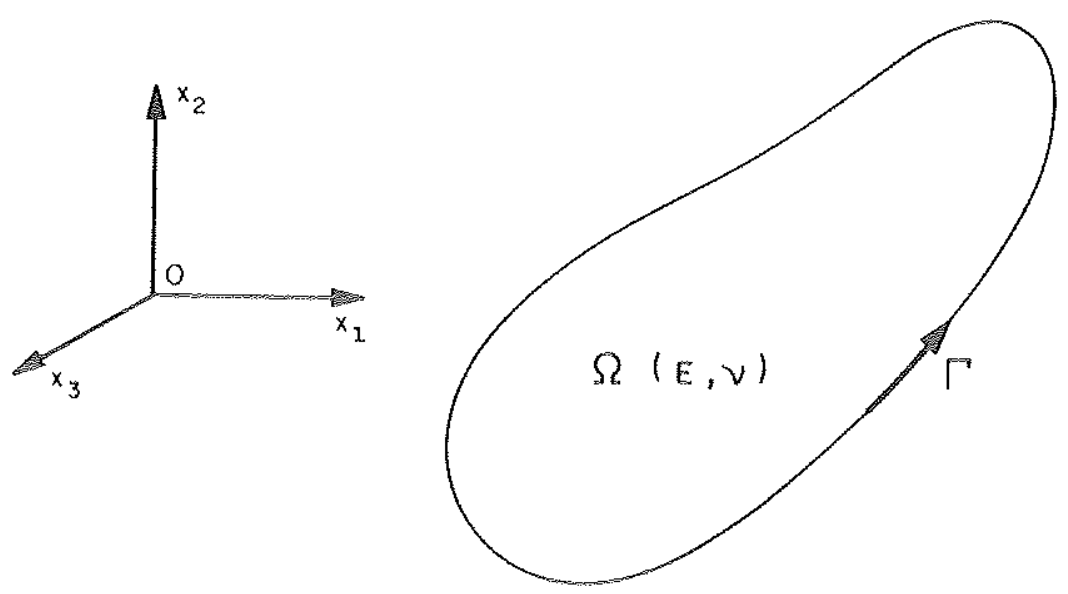

FIGURA 2.I - Corpo tridimensional de dominio $\Omega$ e contorno $\Gamma$.

Com o intuito de se obterem expressoes mais concisas, optou-se, neste item, pelo emprego da notaçào indicial. Desta forma. a representação das coordenadas no sistema cartesiano Oxyz será, agora, $x_{1}, x_{2} \quad e \quad x_{3}$ respectivamente. A convenção utilizada para somatório será:

$$
\begin{aligned}
& a_{1} x_{i}=a_{m} x_{m}=a_{1} x_{1}+a_{2} x_{2}+a_{3} x_{3} \\
& a_{i 1}=a_{m n}=a_{11}+a_{22}+a_{33} \\
& a_{i j} x_{i}=a_{1 j} x_{1}+a_{2 j} x_{2}+a_{3 j} x_{3},
\end{aligned}
$$

para $\mathbf{i}=\mathbf{m}=1,2,3$. 
A notação de derivada parcial, $\partial$, será substituída por uma "vírgula", como nos exemplos:

$$
\begin{aligned}
& \frac{\partial \mathbf{V}}{\partial \mathbf{x}_{\mathbf{j}}}=\mathbf{V}_{\mathbf{i}} \\
& \frac{\partial \mathbf{U}_{\mathbf{i}}}{\partial \mathbf{x}_{\mathbf{i}}}=\mathbf{U}_{\mathbf{i}, \mathbf{j}} \\
& \frac{\partial \sigma_{\mathrm{ij}}}{\partial \mathbf{x}_{\mathrm{i}}}=\sigma_{\mathrm{ij}, \mathbf{j}}
\end{aligned}
$$

O Delta de Kronecker, representado por $\delta_{\mathrm{ij}}$, é definido como:

$$
\delta_{\mathrm{ij}}=\left\{\begin{array}{l}
0, \text { se } \mathrm{i} \neq \mathrm{j} \\
1, \text { se } \mathrm{i}=\mathrm{j}
\end{array}\right.
$$

Impondo-se as condiçòes de equilíbrio estático a um elemento infinitesimal, obtém-se uma equação diferencial de equilíbrio da forma:

$$
\sigma_{i j, j}+b_{i}=0
$$

onde $\sigma_{\mathbf{i j}, \mathbf{j}}$ é o divergente do tensor de tensòes $\mathbf{e} \mathbf{b}_{\mathbf{i}}$ o vetor das forças volumétricas.

As relaçòes cntre as deformações especificas e deslocamentos podem ser expressas por:

$$
\varepsilon_{i \mathrm{i}}=\frac{1}{2}\left[u_{i, j}+u_{j, i}\right]
$$

sendo $\varepsilon_{\mathrm{ij}}$ o tensor das deformaçoes e $\mathbf{u}_{\mathrm{i}, \mathrm{j}}$ a derivada do vetor dos deslocamentos.

Já as relaçòes entre o tensor das tensões c o tensor das deformações, são expressas pela lei de Hooke, na forma:

$$
\sigma_{i j}=\frac{2 G v}{1-2 v} \varepsilon_{k k} \delta_{i j}+2 G \varepsilon_{i j},
$$

ou, na forma inversa,

$$
\varepsilon_{\mathrm{ij}}=\frac{1}{2 \mathrm{G}}\left[\sigma_{\mathrm{ij}}-\frac{v}{1+v} \sigma_{\mathrm{kk}} \delta_{\mathrm{ij}}\right]
$$


As componentes das forças de superficie podem ser representadas em função das componentes de tensão de um ponto da superficie, resultando a relação:

$$
p_{i}=\sigma_{i j} n_{j}
$$

em que $\mathbf{n}_{\mathbf{j}}$ săo os cossenos diretores da normal ao plano tangente à superficic no ponto considerado, como mostra a Fig. 2.2.

Substituindo-se as cquações (2.8) e (2.7) cm (2.6), obtém-se a equação diferencial de equilibrio em termos de deslocamentos, conhecida com equação de Navicr.

$$
u_{1, j i}+\frac{1}{1-2 v} u_{i, j i}+\frac{b_{i}}{G}=0
$$

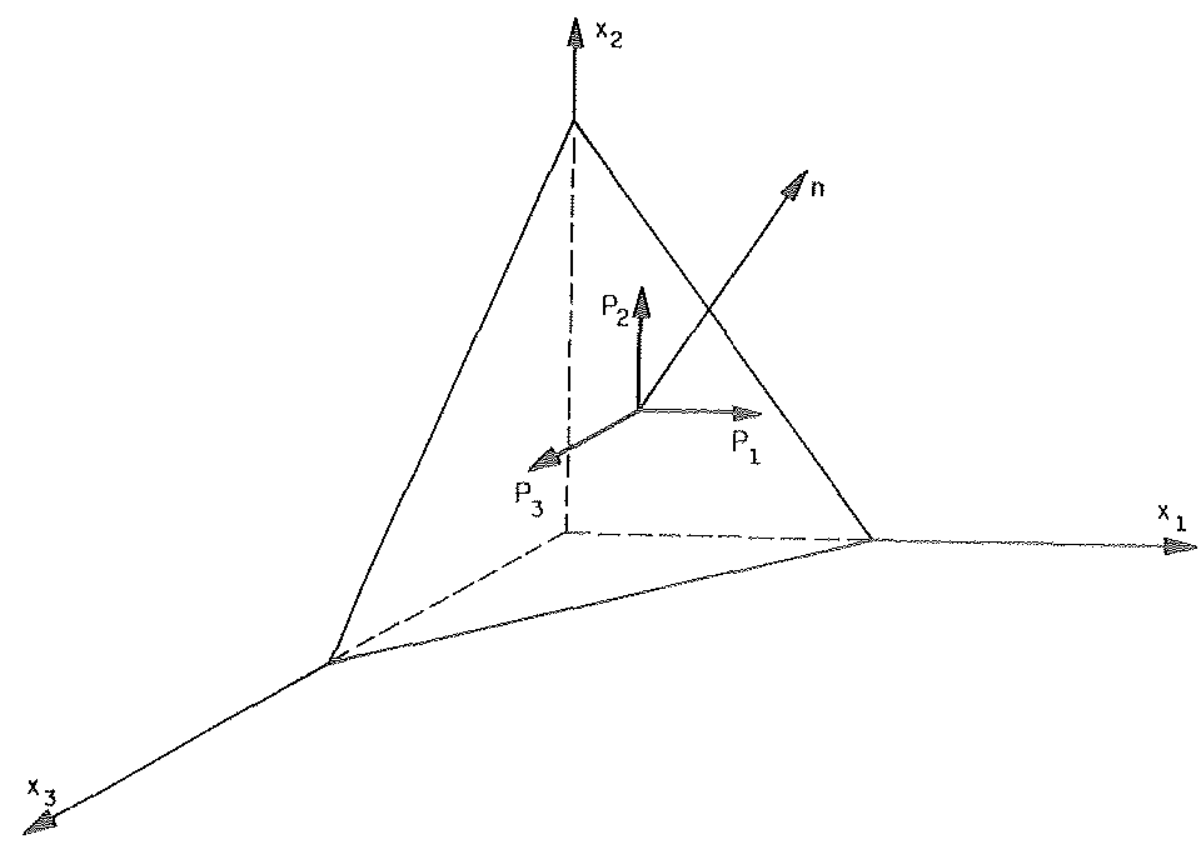

FIGURA 2.2 - Componentes das forças de superfícic no tetraedro de Cauchy. 
As equações de equilibrio estático (2.6), a relação deformação/deslocamento (2.7) e a lei de Hooke (2.8) e (2.9), definem completamente um problema elástico linear, combinado com as condições de contorno prescritas.

Tais condiçõcs para um ponto $Q$, pertencente ao contorno, podem ser escritas como:

$$
\begin{array}{ll}
\mathbf{u}_{\mathrm{i}}(\mathrm{Q})=\overline{\mathbf{u}}_{\mathrm{i}}(\mathrm{Q}), & \mathrm{Q} \in \Gamma_{1}, \\
\mathbf{e} & \\
\mathbf{p}_{\mathrm{i}}(\mathrm{Q})=\overline{\mathbf{p}}_{\mathrm{i}}(\mathrm{Q}), & \mathrm{Q} \in \Gamma_{2}
\end{array}
$$

sendo $\overline{\mathbf{u}}_{\mathbf{1}}$ e $\overline{\mathbf{p}}_{\mathbf{1}}$ as componentes de deslocamentos e forças de superfícic prescritos no contomo, respectivamentc, como mostra a Fig. 2.3.

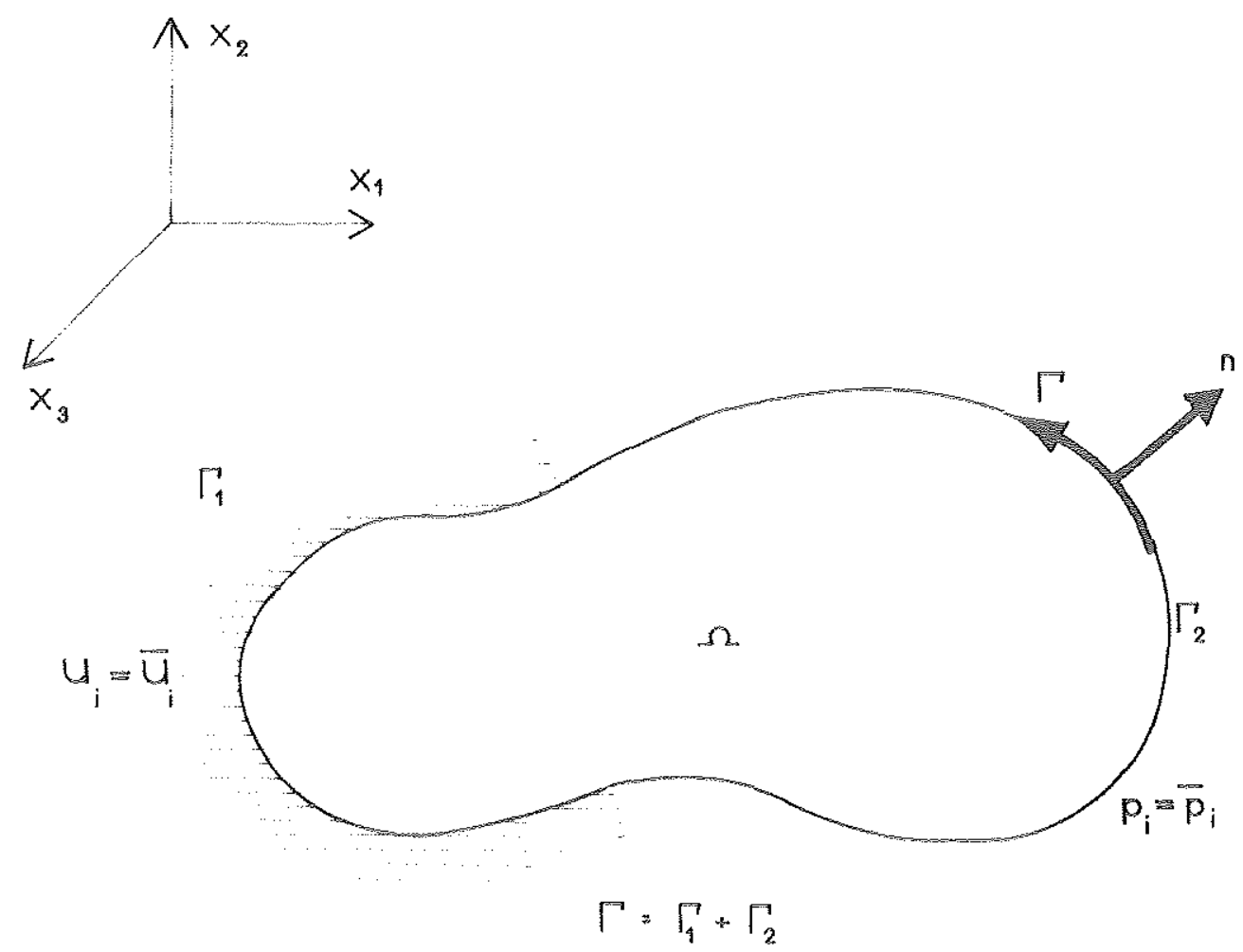

FIGURA 2.3 - Domínio $\Omega$ com o contorno definido pelas condições $\overline{\mathbf{u}}_{\mathbf{i}}$ e $\overrightarrow{\mathbf{p}}_{\mathbf{i}}$ $\mathrm{em} \Gamma_{1} \mathrm{e} \Gamma_{2}$ 


\subsection{SOLUÇÕES FUNDAMENTAIS}

\subsubsection{GENERALIDADES}

Na formulação das equaçòes integrais de contorno para o problema elástico, é indispensável o emprego de uma solução fundamental da equação diferencial $(2,6)$ ou (2.11). Tal soluçăo consiste em se considerar um domínio $\Omega^{*}$, com contorno $\Gamma^{*}$, que contém o corpo de domínio $\Omega$ e contorno $\Gamma$, conforme pode-se ver na Fig. 2.4 .

A solução fundamental ć definida como a resposta em um ponto "q", conhecido como ponto de campo, devido à aplicação de forças concentradas $\mathbf{F}_{\mathbf{i}}{ }^{*}$ no ponto "s", denominado ponto de carga, como mostra a Fig. 2.5 .

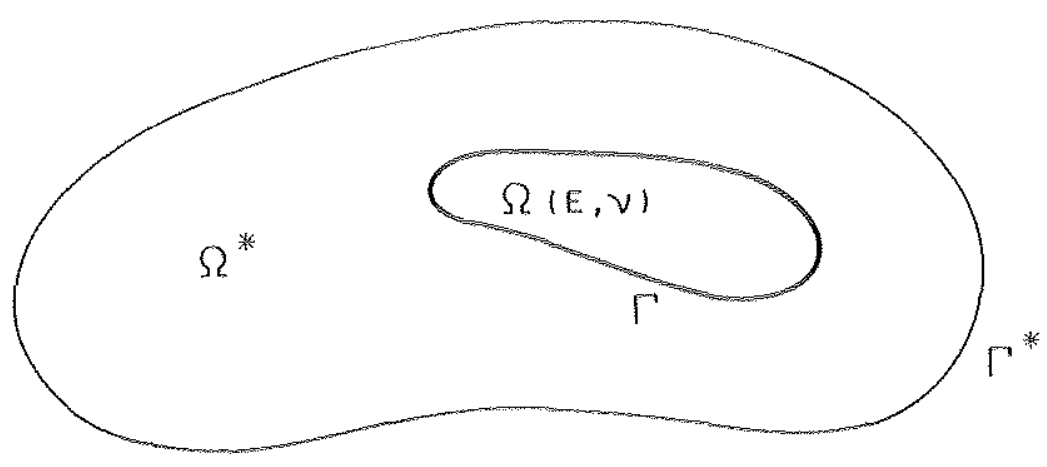

FIGURA 2.4 - Domínio $\Omega$, contido $\mathrm{cm} \Omega^{*}$. 


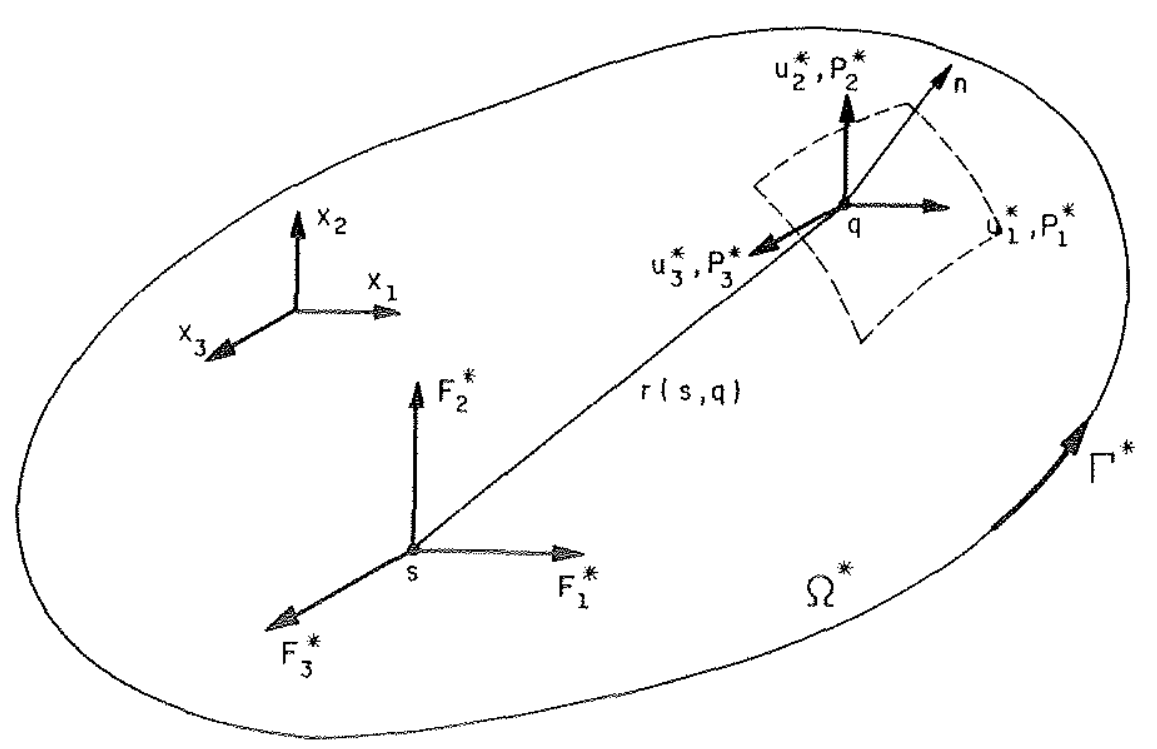

FIGURA 2.5 - Resposta cm "q" devido a uma força $\mathbb{F}_{\mathrm{i}}^{*}$ aplicada $\mathrm{em}$ "s".

O problema é matematicamente formulado através da substituição das forças volumétricas pela cxpressão:

$$
\mathbf{b}_{j}^{*}(q)=\delta(s, q) F_{j}^{*}(s)
$$

sendo que $\delta(s, q)$ é a distribuiçăo Delta de Dirac, definida por:

$$
\begin{aligned}
& \delta(s, q)=0 \text { se } s \neq q \\
& \delta(s, q)=\infty \text { se } s=q ; \text { e } \\
& \int_{\Omega} p(q) \delta(s, q) d \Omega=p(s)
\end{aligned}
$$

sendo que p(s) é uma função qualquer.

Substituindo-se as expressões $(2.13) \mathrm{em}(2.6)$, as equações de equilibrio para o problema fundamental são expressas por:

$$
\sigma_{\mathrm{ij}, \mathrm{j}}^{*}+\delta(s, q) \mathbb{F}_{\mathrm{i}}^{*}(\mathrm{~s})=0
$$

De forma análoga, podem-se obter as demais expressões que governam o problema elástico fundamental. Assim, a equação de Navier do problema fundamental pode scr escrita como: 


$$
U_{i, j i}^{*}+\frac{1}{1-2 v} U_{j, j i}^{*}+\frac{\delta(s, q) F_{i}^{*}(s)}{G}=0
$$

Calculando-se os deslocamentos $\mathbf{u}_{\mathbf{i}}{ }^{*}$ a partir da equação (2.16), as forças de superficic $\mathbf{p}_{\mathbf{i}}^{*}$ podem ser obtidas para uma dada direção definida pelo vetor $\mathbf{n}_{\mathbf{i}}$, a partir da substituição das expressòcs (2.7) $\mathrm{cm} \mathrm{(2.8)} \mathrm{e,} \mathrm{posteriormente,} \mathrm{em} \mathrm{(2.10),}$ resultando $\mathrm{em}$ :

$$
\mathbf{p}_{\mathrm{i}}^{*}=\mathrm{G}\left[\mathbf{U}_{\mathrm{i}, \mathrm{j}}^{*} \mathbf{n}_{\mathrm{j}}+\mathbf{U}_{\mathrm{j}, \mathrm{i}}^{*} \mathbf{n}_{\mathrm{j}}+\frac{2 v}{1-2 v} \mathbf{U}_{\mathrm{j}, \mathrm{i}}^{*} \mathbf{n}_{\mathrm{i}}\right]
$$

Os deslocamentos $\mathbf{u}_{i}$ * c as forças de superficicie $\mathbf{p}_{i}^{*}$ no ponto " $\mathbf{q}$ ", devidos às forças concentradas aplicadas no ponto "s", têm a seguinte representação na forma tensorial:

$$
\begin{aligned}
& u_{i}^{*}(q)=U_{j i}^{*}(s, q) \mathbb{F}_{j}^{*}(s) \\
& p_{i}^{*}(q)=\mathbb{P}_{j i}^{*}(s, q) \mathbb{F}_{j}^{*}(s),
\end{aligned}
$$

sendo que, nos tensores de segunda ordem $\mathbf{U}_{\mathbf{j i}}^{*} \mathrm{c} \mathbb{P}_{\mathbf{j i}}^{*}$, o primeiro índice corresponde à direçăo da carga concentrada e o segundo, à componente do deslocamento ou força de superficic, como mostram as Fig. 2.6 e 2.7.

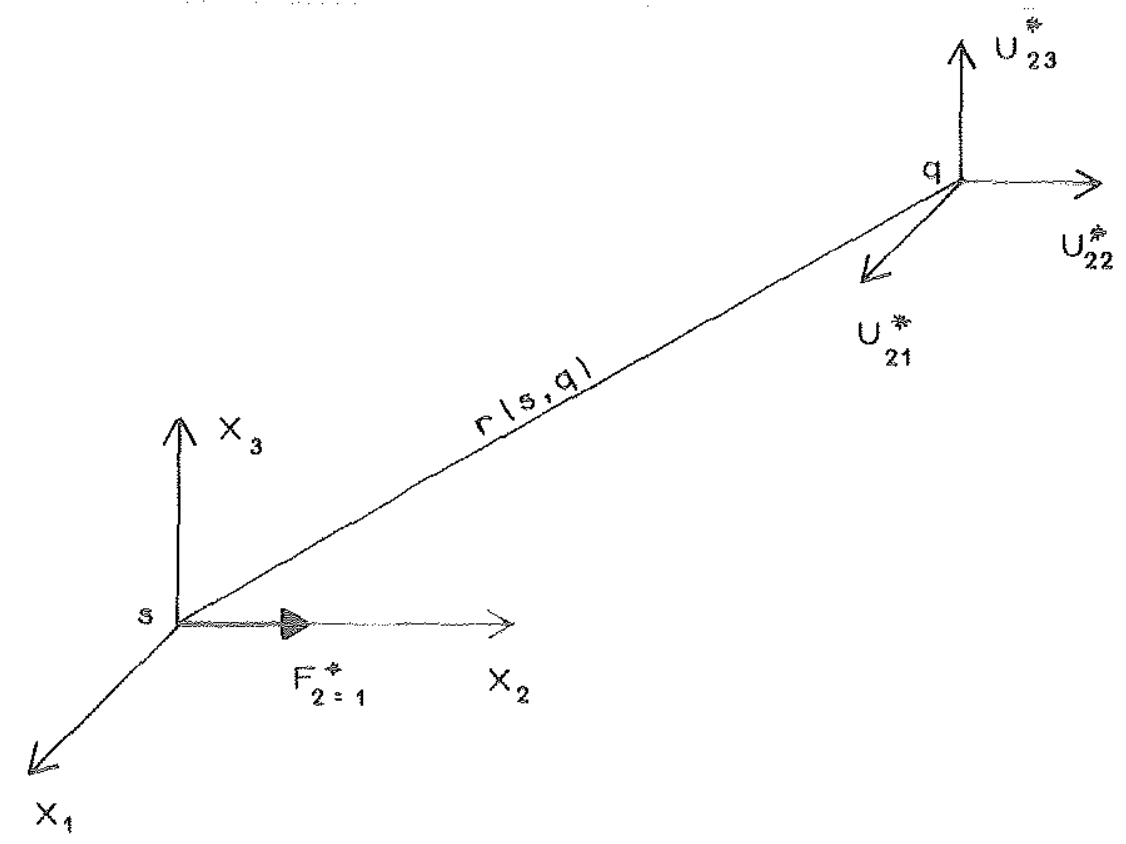

FIGURA 2.6. - Componentes do tensor de deslocamentos fundamentais. 


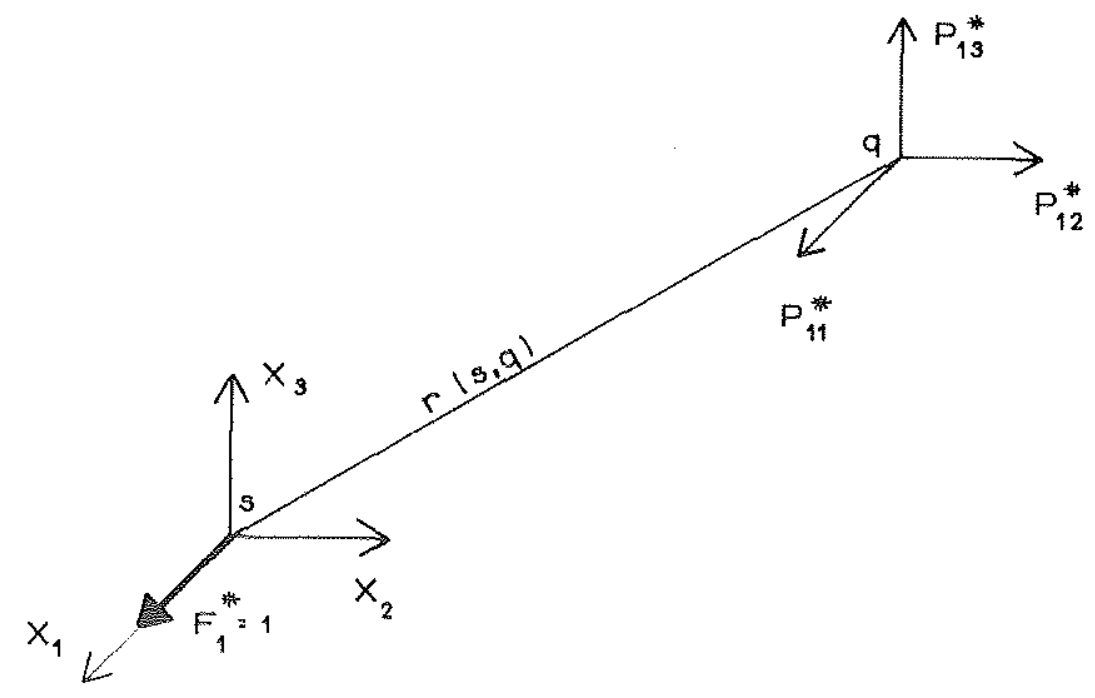

FIGURA 2.7 - Componentes do tensor de forças de superficies fundamentais.

As cxpressòes correspondentes às três direçòes dos eixos cartesianos, dados pelas equaçòes (2.18), podem ser escritas na forma matricial como:

$$
\begin{aligned}
& \mathrm{U}^{*}(q)=\mathbb{\sim}^{* \mathrm{~T}}(s, q) \underset{\sim}{F^{*}}(s) \\
& \mathrm{p}^{*}(q)=\mathrm{P}^{* \mathrm{~T}}(s, q) \mathbb{\sim}^{* *}(s)
\end{aligned}
$$

sendo:

$$
\underset{\sim}{u}(q)=\left\{\begin{array}{l}
u_{1}^{*} \\
u_{2}^{*} \\
u_{3}^{*}
\end{array}\right\},
$$

o vetor das componentes dos deslocamentos no ponto "q":

$$
p^{*}(q)=\left\{\begin{array}{l}
p_{1}^{*} \\
p_{2}^{*} \\
p_{3}^{*}
\end{array}\right\}
$$

o vetor das componentes das forças de superficic no ponto " $q$ ": 


$$
\mathbf{F}^{*}(\mathbf{s})=\left\{\begin{array}{l}
\mathbf{F}_{1}^{*} \\
\mathbf{F}_{2}^{*} \\
\mathbf{F}_{3}^{*}
\end{array}\right\}
$$

o vetor das componentes das forças concentradas atuando no ponto "s",

$$
\underset{\sim}{\mathbf{U}^{*}}(\mathbf{s}, \mathbf{q})=\left[\begin{array}{ccc}
\mathbf{U}_{11}^{*} & \mathrm{U}_{12}^{*} & \mathrm{U}_{13}^{*} \\
\mathbf{U}_{21}^{*} & \mathbf{U}_{22}^{*} & \mathbf{U}_{23}^{*} \\
\mathbf{U}_{31}^{*} & \mathbf{U}_{32}^{*} & \mathbf{U}_{33}^{*}
\end{array}\right]
$$

o tensor dos deslocamentos fundamentais, e

$$
\underset{\sim}{\mathbf{P}^{*}}(\mathbf{s}, \mathbf{q})=\left[\begin{array}{lll}
\mathbf{P}_{11}^{*} & \mathbf{P}_{12}^{*} & \mathbf{P}_{13}^{*} \\
\mathbf{P}_{21}^{*} & \mathbf{P}_{22}^{*} & \mathbf{P}_{23}^{*} \\
\mathbf{P}_{31}^{*} & \mathbf{P}_{32}^{*} & \mathbf{P}_{33}^{*}
\end{array}\right],
$$

o tensor das forças de superficic fundamentais.

$\dot{\mathrm{E}}$ importante ressaltar que, para cada domínio $\Omega^{*}$, assim como para condições de contomo próprias, tem-se uma solução fundamental. Dessa forma, para domínios infinitos, obtem-se a solução de Kelvin, e para domínios semi-infinitos, as soluçōes de Mindlin e Boussinesq-Cerruti.

\subsubsection{SOLUÇÃO FUNDAMENTAL DE KELVIN}

$\mathrm{Na}$ solução fundamental de KELVIN ${ }^{*}$ apud LOVE (1944), o domínio tridimensional $\Omega^{*}$ é considerado infinito, homogèneo c clástico linear, como mostra a Fig. 2.8 .

\footnotetext{
"KELVIN. L. (1863) Phil. Trans. Roy. Soc., v. 153 apud LOVE. A.E.H. (1944) A treatise on the mathematical theory of elasticity. New York. Dover Publications.
} 


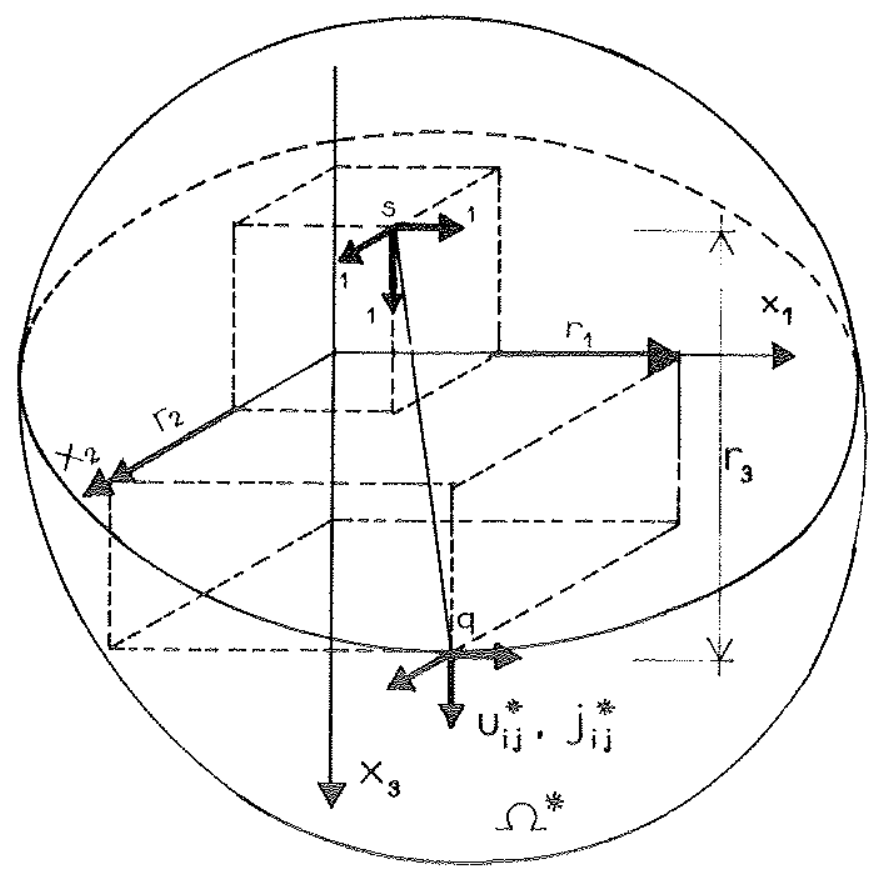

FIGURA 2.8 - Problema Fundamental de Kelvin.

As expressōes para deslocamentos e forças de superficie fundamentais, $\mathrm{cm}$ termos dos respectivos tensores, de acordo com BREBBIA (1978b), são dadas por:

$$
\begin{aligned}
& U_{i j}^{*}(s, q)=\frac{1+v}{8 \pi E(1-v) r}\left[(3-4 v) \delta_{i j}+r, i, i\right] \\
& P_{i j}^{*}(s, q)=\frac{-1}{8 \pi(1-v) r^{2}}\left\{r_{, i} n_{i}\left[(1-2 v) \delta_{i j}+3 r_{, i}{ }^{r}, j\right]+\right. \\
& \left.-(1-2 v)\left(n_{i, i}{ }^{*}-n_{i} i^{*}, j\right)\right\}
\end{aligned}
$$

em que, $r=r(s, q)$ é a distância entre os pontos " $s$ " e " $q$ ", sendo dada por:

$$
r=\left[\begin{array}{ll}
r_{i} & r_{i}
\end{array}\right]^{1 / 2} ; \quad r_{i}=x_{i}(q)-x_{i}(s)
$$

Note que todas as derivadas são relativas ao ponto " $q$ ", ou seja, ponto onde são observados os efeitos considerados. Assim, tem-se:

$$
r_{i}=\frac{\partial r}{\partial x_{i}(q)}=\frac{r_{i}}{r}
$$




\subsubsection{SOLUÇÃO FUNDAMENTAL DE MINDLIN}

A solução fundamental de MINDLIN (1936) è empregada para domínios semi-infinitos homogèneos, clásticos-lineares. Nestas condições, o plano $\mathbf{x}_{\mathbf{3}}=\mathbf{0}$ é considerado como superficie do contorno $\bar{\Gamma}$ e admitido como sendo livre de forças de superficie. O ponto " $\mathrm{s}$ " de aplicação de força $\mathbf{F}_{\mathrm{i}}^{*}$ ' $\mathrm{e}$ um qualquer do interior deste domínio, c o ponto "s" seu simétrico em relação ao plano do contorno $\bar{\Gamma}$, como mostra a Fig. 2.9.

Neste caso, as expressoes para as componentes do tensor de deslocamentos podem ser escritas como:

$$
\begin{aligned}
& U_{11}^{*}=K_{d}\left\{\frac{3-4 v}{r}+\frac{1}{R}+\frac{r_{1}^{2}}{r^{3}}+\frac{(3-4 v) r_{1}^{2}}{R^{3}}+\frac{2 C Z}{R^{3}}\left(1-\frac{3 r_{1}^{2}}{R^{2}}\right)+\right. \\
& \left.+\frac{4(1-v)(1-2 v)}{R+R_{3}}\left[1-\frac{r_{1}^{2}}{R\left(R+R_{3}\right)}\right]\right\} \\
& U_{12}^{*}=K_{d} r_{1} r_{2}\left\{\frac{1}{r^{3}}+\frac{3-4 v}{R^{3}}-\frac{6 C Z}{R^{5}}-\frac{4(1-v)(1-2 v)}{R\left(R+R_{3}\right)^{2}}\right\} \\
& U_{13}^{*}=K_{1} r_{1}\left\{\frac{r_{3}}{r^{3}}+\frac{(3-4 v) r_{3}}{R^{3}}-\frac{6 C Z R_{3}}{R^{5}}+\frac{4(1-v)(1-2 v)}{R\left(R+R_{3}\right)}\right\} \\
& U_{21}^{*}=U_{12}^{*} \\
& U_{22}^{*}=\mathbb{K}_{\mathrm{d}}\left\{\frac{3-4 v}{r}+\frac{1}{\mathbb{R}}+\frac{r_{2}^{2}}{r^{3}}+\frac{(3-4 v) r_{2}^{2}}{R^{3}}+\frac{2 C Z}{\mathbb{R}^{3}}\left(1-\frac{3 r_{2}^{2}}{R^{2}}\right)+\right. \\
& \left.+\frac{4(1-v)(1-2 v)}{R+R_{3}}\left[1-\frac{r_{2}^{2}}{R\left(R+R_{3}\right)}\right]\right\} \\
& U_{23}^{*}=\frac{\mathbb{r}_{2}}{r_{1}} U_{13}^{*} \\
& U_{31}^{*}=K_{d} \mathbb{l}_{1}\left\{\frac{r_{3}}{r^{3}}+\frac{(3-4 v) r_{3}}{\mathbb{R}^{3}}-\frac{4(1-v)(1-2 v)}{\mathbb{R}\left(\mathbb{R}+\mathbb{R}_{3}\right)}+\frac{6 C Z R_{3}}{\mathbb{R}^{5}}\right\}
\end{aligned}
$$




$$
\begin{aligned}
U_{32}^{*}= & \frac{r_{2}}{r_{1}} U_{31}^{*} \\
U_{33}^{*}= & K_{d}\left\{\frac{3-4 v}{r}+\frac{8(1-v)^{2}-(3-4 v)}{r}+\frac{r_{3}^{2}}{r^{3}}+\frac{(3-4 v) R_{3}^{2}-2 C Z}{R^{3}}+\right. \\
& \left.+\frac{6 C Z R_{3}^{2}}{R^{5}}\right\}
\end{aligned}
$$

sendo:

$$
\begin{aligned}
& r=\left(\begin{array}{ll}
r_{i} & r_{i}
\end{array}\right)^{1 / 2} \\
& R=\left(\mathbb{R}_{i} \quad R_{i}\right)^{1 / 2} \\
& r_{i}=x_{i}(q)-x_{i}(s) \\
& R_{i}=x_{i}(q)-x_{i}\left(s^{q}\right) \\
& C=x_{3}(s)>0 \\
& Z{ }^{\prime}=x_{3}(q)>0 \\
& K_{d}=\frac{1+v}{8 \pi C(1-v)} \\
& \mathbb{K}_{s}=\frac{1}{8 \pi(1-v)}
\end{aligned}
$$




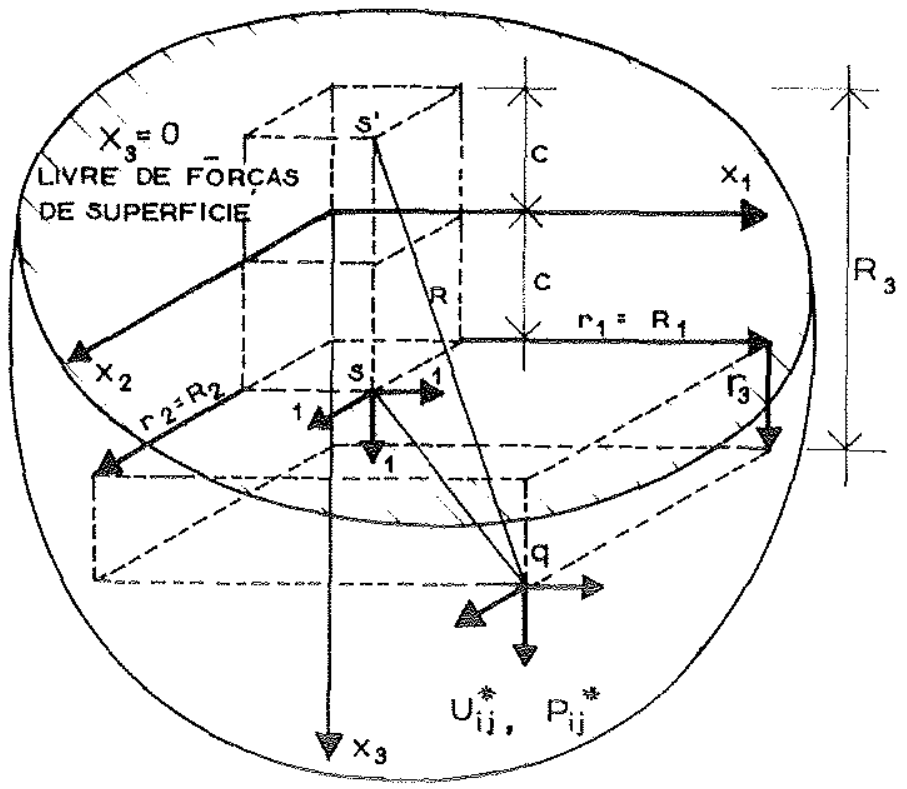

$\Omega^{*}$

FIGURA 2.9 - Problema fundamental de Mindlin.

As expressoes para forças de superficie são obtidas através da equação,

$$
\mathbb{P}_{i j}^{*}=\sigma_{j k}^{* i} n_{k} \text {, }
$$

isto é, em função do tensor de terceira ordem das tensòes e das componentes do vetor normal à superfícic no ponto " $q "$.

A seguir, são apresentadas as vinte e sete componentes do tensor de terceira ordem das tensōes, necessárias à obtenção do tensor das forças de superfícic.

Expressões para as forças de superficic:

$$
\begin{aligned}
\sigma_{11}^{*_{1}}= & \mathbb{K}_{s} \mathbf{r}_{1}\left\{-\frac{1-2 v}{\mathbf{r}^{3}}+\frac{(1-2 v)(5-4 v)}{\mathbb{R}^{3}}-\frac{3 \mathbf{r}_{1}^{2}}{\mathbf{r}^{5}}-\frac{3(3-4 v) \mathbf{r}_{1}^{2}}{\mathbb{R}^{5}}+\right. \\
& -\frac{4(1-v)(1-2 v)}{\mathbb{R}\left(\mathbb{R}+\mathbb{R}_{3}\right)^{2}}\left[3-\frac{\mathbf{r}_{1}^{2}\left(3 \mathbb{R}+\mathbb{R}_{3}\right)}{\mathbb{R}^{2}\left(\mathbb{R}+\mathbb{R}_{3}\right)}\right]+ \\
& \left.+\frac{6 C}{\mathbb{R}^{5}}\left[3 \mathbb{C}-(3-2 v) \mathbb{R}_{3}+\frac{5 r_{1}^{2} \mathbb{Z}}{\mathbb{R}^{2}}\right]\right\}
\end{aligned}
$$




$$
\begin{aligned}
& \sigma_{12}^{*_{1}}=\mathbf{K}_{\mathrm{s}} \mathbf{r}_{2}\left\{-\frac{1-2 v}{\mathbf{r}^{3}}+\frac{1-2 v}{\mathbf{R}^{3}}-\frac{3 \mathbf{r}_{1}^{2}}{\mathbf{r}^{5}}-\frac{3(3-4 v) \mathbf{r}_{1}^{2}}{\mathbf{R}^{5}}+\right. \\
& \left.-\frac{4(1-v)(1-2 v)}{R\left(R+R_{3}\right)^{2}}\left[1-\frac{r_{1}^{2}\left(3 R+R_{3}\right)}{R^{2}\left(R+R_{3}\right)}\right]-\frac{6 C Z}{R^{5}}\left(1-\frac{5 r_{1}^{2}}{R^{2}}\right)\right\} \\
& \sigma_{13}^{* 1}=\mathbf{K}_{s}\left\{-\frac{(1-2 v) r_{3}}{\mathbf{r}^{3}}+\frac{(1-2 v) r_{3}}{\mathbf{R}^{3}}-\frac{3 \mathbf{r}_{1}^{2} \mathbf{r}_{3}}{\mathbf{r}^{5}}-\frac{3(3-4 v) \mathbf{r}_{1}^{2} \mathbf{R}_{3}}{\mathbf{R}^{5}}+\right. \\
& \left.-\frac{6 C}{R^{5}}\left[Z_{3}-(1-2 v) r_{1}^{2}-\frac{5 r_{1}^{2} Z R_{3}}{R^{2}}\right]\right\} \\
& \sigma_{21}^{* 1}=\sigma_{12}^{* 1} \\
& \sigma_{22}^{* 1}=\mathbb{K}_{s} \mathbf{r}_{1}\left\{\frac{1-2 v}{r^{3}}+\frac{(1-2 v)(3-4 v)}{\mathbb{R}^{3}}-\frac{3 r_{2}^{2}}{r^{5}}-\frac{3(3-4 v) r_{2}^{2}}{\mathbb{R}^{5}}+\right. \\
& \left.-\frac{4(1-v)(1-2 v)}{\mathbb{R}\left(\mathbb{R}+\mathbb{R}_{3}\right)^{2}}\left[1-\frac{\mathbf{r}_{2}^{2}\left(3 \mathbb{R}+\mathbb{R}_{3}\right)}{\mathbb{R}^{2}\left(\mathbb{R}+\mathbb{R}_{3}\right)}\right]+\frac{6 C}{\mathbb{R}^{5}}\left[\mathbf{C}-(1-2 v) \mathbb{R}_{3}+\frac{5 \mathbf{r}_{2}^{2} \mathbb{Z}}{\mathbb{R}^{2}}\right]\right\} \\
& \sigma_{23}^{*_{1}}=\mathbb{K}_{\mathrm{s}} \mathbf{r}_{1} \mathbf{r}_{2}\left\{-\frac{3 \mathbf{r}_{3}}{\mathbf{r}^{5}}-\frac{3(3-4 v) \mathbb{R}_{3}}{\mathbb{R}^{5}}+\frac{6 C}{\mathbb{R}^{5}}\left(1-2 v+\frac{5 \mathbb{Z} \mathbb{R}_{3}}{\mathbb{R}^{2}}\right)\right\} \\
& \sigma_{31}^{* 1}=\sigma_{13}^{* 1} \\
& \sigma_{32}^{* 1}=\sigma_{23}^{* 1} \\
& \sigma_{33}^{* 1}=\mathbb{K}_{s} \mathbf{r}_{1}\left\{\frac{1-2 v}{\mathbf{r}^{3}}-\frac{1-2 v}{\mathbb{R}^{3}}-\frac{3 \mathbf{r}_{3}^{2}}{\mathbf{r}^{5}}-\frac{3(3-4 v) \mathbb{R}_{3}^{2}}{\mathbb{R}^{5}}+\right. \\
& \left.+\frac{6 \mathbf{C}}{\mathbb{R}^{5}}\left[\mathbf{C}+(1-2 v) \mathbb{R}_{3}+\frac{5 \mathbb{Z} \mathbb{R}_{3}^{2}}{\mathbb{R}^{2}}\right]\right\} \\
& \sigma_{11}^{* 2}=\mathbb{K}_{s} \mathbf{r}_{2}\left\{\frac{1-2 v}{\mathbf{r}^{3}}+\frac{(1-2 v)(3-4 v)}{\mathbb{R}^{3}}-\frac{3 \mathbf{r}_{1}^{2}}{\mathbf{r}^{5}}-\frac{3(3-4 v) r_{1}^{2}}{\mathbb{R}^{5}}+\right. \\
& \left.-\frac{4(1-v)(1-2 v)}{\mathbb{R}\left(\mathbb{R}+\mathbb{R}_{3}\right)^{2}}\left[1-\frac{\mathbf{r}_{1}^{2}\left(3 \mathbb{R}+\mathbb{R}_{3}\right)}{\mathbb{R}^{2}\left(\mathbb{R}+\mathbb{R}_{3}\right)}\right]+\frac{6 C}{\mathbb{R}^{5}}\left[C-(1-2 v) \mathbb{R}_{3}+\frac{5 \mathbf{r}_{1}^{2} \mathbb{Z}}{\mathbb{R}^{2}}\right]\right\}
\end{aligned}
$$




$$
\begin{aligned}
& \sigma_{12}^{* 2}=K_{s} r_{1}\left\{-\frac{1-2 v}{r^{3}}+\frac{1-2 v}{R^{3}}-\frac{3 r_{2}^{2}}{r^{5}}-\frac{3(3-4 v) r_{2}^{2}}{R^{5}}+\right. \\
& \left.-\frac{4(1-v)(1-2 v)}{R\left(R+R_{3}\right)^{2}}\left[1-\frac{r_{2}^{2}\left(3 R+R_{3}\right)}{R^{2}\left(R+R_{3}\right)}\right]-\frac{6 C Z}{R^{5}}\left(1-\frac{5 r_{2}^{2}}{R^{2}}\right)\right\} \\
& \sigma_{13}^{* 2}=\sigma_{23}^{* 1} \\
& \sigma_{21}^{* 2}=\sigma_{12}^{* 2} \\
& \sigma_{22}^{\star 2}=\mathbf{K}_{\mathrm{s}} \mathbf{r}_{2}\left\{-\frac{(1-2 v)}{\mathbf{r}^{3}}+\frac{(1-2 v)(5-4 v)}{\mathbf{R}^{3}}-\frac{3 \mathbf{r}_{2}^{2}}{\mathbf{r}^{5}}-\frac{3(3-4 v) \mathbf{r}_{2}^{2}}{\mathbf{R}^{5}}+\right. \\
& \left.-\frac{4(1-v)(1-2 v)}{R\left(R+R_{3}\right)^{2}}\left[3-\frac{\mathbf{r}_{2}^{2}\left(3 \mathbb{R}+\mathbf{R}_{3}\right)}{\mathbb{R}^{2}\left(\mathbf{R}+\mathbf{R}_{3}\right)}\right]+\frac{6 C}{\mathbf{R}^{5}}\left[3 C-(3-2 v) \mathbb{R}_{3}+\frac{5 \mathbf{r}_{2}^{2} \mathbb{Z}}{\mathbb{R}^{2}}\right]\right\} \\
& \sigma_{23}^{* 2}=\mathbb{K}_{s}\left\{-\frac{(1-2 v) r_{3}}{\mathbf{r}^{3}}+\frac{(1-2 v) r_{3}}{\mathbb{R}^{3}}-\frac{3 r_{2}^{2} \mathbf{r}_{3}}{r^{5}}-\frac{3(3-4 v) \mathbf{r}_{2}^{2} \mathbb{R}_{3}}{\mathbf{R}^{5}}+\right. \\
& \left.-\frac{6 C}{\mathbb{R}^{5}}\left[\mathbb{Z R}_{3}-(1-2 v) \mathrm{r}_{2}^{2}-\frac{5 \mathbf{r}_{2}^{2} \mathbb{Z} \mathbb{R}_{3}}{\mathbb{R}^{2}}\right]\right\} \\
& \sigma_{31}^{* 2}=\sigma_{13}^{* 2} \\
& \sigma_{32}^{* 2}=\sigma_{23}^{* 2} \\
& \sigma_{33}^{* 2}=\frac{r_{2}}{r_{1}} \sigma_{33}^{* 1} \\
& \sigma_{11}^{* 3}=\mathbb{K}_{\mathrm{s}}\left\{\frac{(1-2 v) r_{3}}{\mathbf{r}^{3}}-\frac{3 \mathbf{r}_{1}^{2} \mathbf{r}_{3}}{\mathbf{r}^{5}}+\frac{(1-2 v)\left(3 \mathbf{r}_{3}-4 v \mathbb{R}_{3}\right)}{\mathbb{R}^{3}}+\right. \\
& -\frac{3(3-4 v) r_{1}^{2} r_{3}-6 C \mathbb{R}_{3}[(1-2 v) \mathbb{Z}-2 v \mathbb{C}]}{\mathbb{R}^{5}}-\frac{30 \mathrm{Cr}_{1}^{2} \mathbb{Z R}_{3}}{\mathbb{R}^{7}}+ \\
& \left.-\frac{4(1-v)(1-2 v)}{\mathbb{R}\left(\mathbb{R}+\mathbb{R}_{3}\right)}\left(1-\frac{\mathbf{r}_{1}^{2}}{\mathbb{R}\left(\mathbb{R}+\mathbb{R}_{3}\right)}-\frac{\mathbf{r}_{1}^{2}}{\mathbb{R}^{2}}\right)\right\}
\end{aligned}
$$




$$
\begin{aligned}
& \sigma_{12}^{* 3}=K_{s} r_{1} r_{2}\left\{-\frac{3 r_{3}}{r^{5}}-\frac{3(3-4 v) r_{3}}{R^{5}}+\frac{4(1-v)(1-2 v)}{R^{2}\left(R+R_{3}\right)}\left(\frac{1}{R+R_{3}}+\frac{1}{R}\right)+\right. \\
& \left.-\frac{30 \mathrm{CZR}_{3}}{\mathbf{R}^{7}}\right\} \\
& \sigma_{13}^{* 3}=K_{s} r_{1}\left\{-\frac{1-2 v}{r^{3}}+\frac{1-2 v}{R^{3}}-\frac{3 r_{3}^{2}}{r^{5}}-\frac{3(3-4 v) Z_{R_{3}}-3 C(3 Z+C)}{R^{5}}+\right. \\
& \left.-\frac{30 \mathbf{C Z R} \mathbf{R}_{3}^{2}}{\mathbf{R}^{7}}\right\} \\
& \sigma_{21}^{* 3}=\sigma_{12}^{* 3} \\
& \sigma_{22}^{* 3}=K_{s}\left\{\frac{(1-2 v) r_{3}}{r^{3}}-\frac{3 r_{2}^{2} r_{3}}{r^{5}}+\frac{(1-2 v)\left(3 r_{3}-4 v R_{3}\right)}{\mathbb{R}^{3}}+\right. \\
& -\frac{3(3-4 v) \mathbf{r}_{2}^{2} \mathbf{r}_{3}-6 \mathbb{C R}_{3}[(1-2 v) \mathbb{Z}-2 v C]}{\mathbb{R}^{5}}-\frac{30 \mathrm{Cr}_{2}^{2} \mathbb{Z} \mathbb{R}_{3}}{\mathbb{R}^{7}}+ \\
& \left.-\frac{4(1-v)(1-2 v)}{\mathbf{R}\left(\mathbb{R}+\mathbb{R}_{3}\right)}\left[1-\frac{\mathbf{r}_{2}^{2}}{\mathbb{R}\left(\mathbb{R}+\mathbb{R}_{3}\right)}-\frac{\mathbf{r}_{2}^{2}}{\mathbb{R}^{2}}\right]\right\} \\
& \sigma_{23}^{* 3}=\mathbb{K}_{s{ }^{1}}\left\{-\frac{1-2 v}{\mathbf{r}^{3}}+\frac{1-2 v}{\mathbb{R}^{3}}-\frac{3 \mathbf{r}_{3}^{2}}{\mathbf{r}^{5}}-\frac{3(3-4 v) Z \mathbb{R}_{3}-3 \mathbb{C}(3 \mathbb{Z}+\mathbb{C})}{\mathbb{R}^{5}}+\right. \\
& \left.-\frac{30 C \mathbb{Z} \mathbb{R}_{3}^{2}}{\mathbb{R}^{7}}\right\} \\
& \sigma_{31}^{* 3}=\sigma_{13}^{* 3} \\
& \sigma_{32}^{* 3}=\sigma_{23}^{* 3} \\
& \sigma_{33}^{* 3}=K_{s}\left\{-\frac{(1-2 v) r_{3}}{r^{3}}+\frac{(1-2 v) r_{3}}{\mathbb{R}^{3}}-\frac{3 r_{3}^{3}}{r^{5}}+\right. \\
& \left.-\frac{3(3-4 v) Z \mathbb{R}_{3}^{2}-3 C \mathbb{R}_{3}(5 \mathbb{Z}-C)}{\mathbf{R}^{5}}-\frac{30 C \not \mathbb{R}_{3}^{3}}{\mathbb{R}^{7}}\right\}
\end{aligned}
$$


Como mostrado na Fig. 2.9, C representa a distancia entre o ponto de carga "s" e o plano de contorno $\mathbf{x}_{3}=\mathbf{0}$.

Note-se que ao fazer $\mathbf{C}$ tender ao infinito $(\mathbf{C} \rightarrow \infty)$, isto $\dot{\mathrm{e}}$, os pontos "s" $\mathrm{c}$ " $q$ " se afastarem do plano de contorno $\widetilde{\Gamma}\left(x_{3}=0\right)$, mantendo-se $r(s, q)$ inalterada, a influência do contorno desaparece, chegando-se à solução fundamental de Kelvin.

Procedendo de forma análoga, fazendo agora $\mathbf{C}$ tendendo a zero $(\mathbf{C} \rightarrow \mathbf{0})$, o ponto "s" de carga estará situado no plano de contorno $\bar{\Gamma}$, obtendo-se a solução fundamental de Boussinesq-Cerruti, cujas expressòes serăo apresentadas no próximo item.

Uma formulaçào para a soluçào fundamental de Mindlin semethante à de Kelvin, cm notação indicial, pode ser encontrada cm SÁ \& TELLES (1986), a qual é indicada para utilizaçòes computacionais, bem como cm BREBBIA et al. (1984). Nesta formulação, a soluçào fundamental de Mindlin pode ser escrita como a soma da solução fundamental de Kelvin com uma parcela dita complementar, tomando a seguinte forma:

$$
()^{*}=()^{k}+()^{c} \text {. }
$$

sendo que, ()$^{\mathbf{k}}$ e ()$^{\mathbf{c}}$, representam as expressòes de Kelvin e complementares, respectivamente.

\subsubsection{SOLUÇÃO FUNDAMENTAL DE BOUSSINESQ-CERRUTII}

As soluçōes fundamentais de BOUSSINESQ (1885), para força normal e CERRUTI (1882), para força tangencial, aplicadas no plano de contorno $\bar{\Gamma}\left(\mathrm{x}_{\mathbf{3}}=\mathbf{0}\right)$, de um domínio $\Omega^{*}$, semi-inifinito, clástico-linear c homogêneo, podem ser cncaradas como um caso particular da solução de Mindlin, admitindo-se o ponto "s", na superficic livre de forças $\left(x_{3}=0\right)$, como mostra a Fig. 2.10. A partir das equaçòes (2.22), podem ser obtidas as expressões para deslocamentos fundamentais da solução de Boussinesq-Cerruti, fazendo $\sim \mathrm{se}=\mathbf{0}, \mathbf{R}=\mathbf{r} \subset \mathbf{R}_{\mathbf{3}}=\mathbf{r}_{\mathbf{3}}$. As componentes da solução fundamental para deslocamentos, são dadas por: 


$$
\begin{aligned}
& \mathbf{U}_{11}^{*}=\mathbf{K}\left[(1-v)+v \mathbf{r}_{, 1}^{2}\right] \\
& \mathbf{U}_{12}^{*}=\mathbf{K v} \mathbf{r}_{1,} \mathbf{r}_{, 2} \\
& \mathbf{U}_{13}^{*}=\mathbf{K}(0,5-v) r_{, 1} \\
& \mathbf{U}_{22}^{*}=\mathbf{K}\left[(1-v)+v r_{, 2}^{2}\right] \\
& \mathbf{U}_{23}^{*}=\mathbf{K}(0,5-v) r_{, 2} \\
& \mathbf{U}_{33}^{*}=\mathbf{K}(1-v) \\
& U_{21}^{*}=U_{12}^{*} ; U_{32}^{*}=-U_{23}^{*} ; U_{31}^{*}=-U_{13}^{*}
\end{aligned}
$$

em que:

$$
\mathbb{K}=\frac{1}{2 \pi \mathbf{G r}}
$$

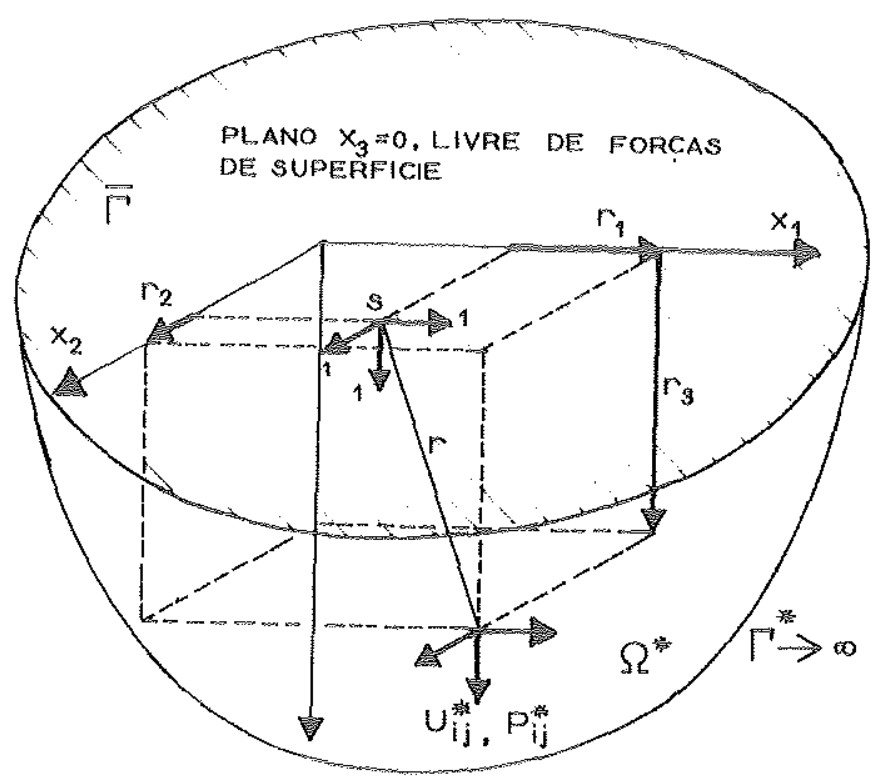

FIGURA 2.10 - Problema Fundamental de Boussinesq Cerruti. 


\subsection{EQUAÇÕES INTEGRAIS PARA PONTOS NO DOMÍNIO}

As equaçòes integrais de deslocamentos para pontos pertencentes ao domínio de um corpo tridimensional, clástico-linear, podem ser obtidas a partir da Técnica dos Resíduos Ponderados ou do Teorema da Reciprocidade (Teorema de Betti).

Considera-se um domínio elástico-linear, isótropo e homogêneo $\Omega$, com contorno $\Gamma$ constituido de dois segmentos $\Gamma_{1}$ e $\Gamma_{2}$, nos quais estão prescritos os deslocamentos (ou condiçăo essencial) e forças de superficie (ou condição natural), respectivamente, sendo $\Gamma=\Gamma_{1}+\Gamma_{2}$, conforme mostrado à Fig. 2.3 .

As representaçocs integrais de deslocamentos para pontos do domínio $\mathbf{\Omega}$ podem ser obtidas a partir dá equação (2.6), utilizando-se a técnica dos resíduos ponderados (BREBBIA, 1978a), sendo que a função ponderadora $\mathbf{w}_{\mathbf{i}}$ é substituída pelas soluçòes fundamentais para deslocamentos e forças de superfície, $\mathbf{u}_{\mathbf{i}}{ }^{*} \mathfrak{c} \mathbf{p}_{\mathbf{i}}{ }^{*}$, respectivamente, obtendo-se a seguinte expressão:

$$
\int_{\Omega}\left(\sigma_{i j, j}+b_{i}\right) u_{i}^{*} d \Omega=\int_{\Gamma_{i}}\left(\bar{u}_{i}-u_{i}\right) p_{i}^{*} d \Gamma+\int_{\Gamma_{2}}\left(p_{i}-\bar{p}_{i}\right) u_{i}^{*} d \Gamma
$$

Integrando-se por partes duas vezes a eq. (2.26), resulta:

$$
\begin{aligned}
\int_{\Omega} b_{i} u_{i}^{*} d \Omega+\int_{\Omega} \sigma_{i j, j}^{*} u_{i} d \Omega= & -\int_{\Gamma_{2}} \bar{p}_{i} u_{i}^{*} d \Gamma-\int_{\Gamma_{1}} p_{i} u_{i}^{*} d \Gamma+\int_{\Gamma_{1}} \bar{u}_{1} p_{i}^{*} d \Gamma+ \\
& +\int_{\Gamma_{2}} u_{i} p_{i}^{*} d \Gamma
\end{aligned}
$$

Agrupando-se as parcelas correspondentes aos segmentos $\Gamma_{1}$ e $\Gamma_{2}$ do contorno $\Gamma$, esta equação pode ser reescrita como:

$$
\begin{aligned}
& -\int_{\Omega} \sigma_{i, j}^{*}(q) u_{i}(q) d \Omega(q)+\int_{\Gamma} p_{i}^{*}(Q) u_{i}(Q) d \Gamma(Q)= \\
& \int_{\Omega} u_{i}^{*}(q) b_{i}(q) d \Omega(q)+\int_{\Gamma} u_{i}^{*}(Q) p_{i}(Q) d \Gamma(Q)
\end{aligned}
$$

sendo " $q$ " um ponto do domínio $\Omega$ c " $Q$ " um ponto do contomo $\Gamma$.

Substituindo-se na eq. (2.28) o termo diferencial do tensor de tensồes dado na eq. (2.15) e fazendo-se uso da terceira propricdade apresentada na eq. (2.14) e as eq. 
(2.18), e tomando-se cada componente $\mathbf{F}_{\mathbf{i}}{ }^{*}$, como independente e igual a unidade, obtém-se a expressão:

$$
\begin{aligned}
u_{i}(s)= & -\int_{\Gamma} P_{i j}^{*}(s, Q) u_{j}(Q) d \Gamma(Q)+\int_{\Gamma} U_{i j}^{*}(s, Q) p_{j}(Q) d \Gamma(Q)+ \\
& +\int_{\Omega} U_{i j}^{*}(s, q) b_{j}(q) d \Omega(q)
\end{aligned}
$$

sendo que os pontos "s" c "q" pertencem ao domínio $\Omega$, e " $Q$ " é um ponto do contorno $\Gamma$.

A eq. (2.29) é conhecida como Identidade SOMIGLIANA (1886) e fornece as componentes de deslocamentos para os pontos "s" no interior do domínio $\Omega$, segundo os eixos de referencia, uma vez que $\mathbf{u}_{\mathbf{j}} \mathbf{e} \mathbf{p}_{\mathbf{j}}$ sejam conhecidos para todos os pontos do contorno.

\subsection{EQUAÇÕES INTEGRAIS PARA PONTOS NO CONTORNO}

A identidade Somigliana fornece apenas os deslocamentos para pontos no interior do domínio.

Necessita-se, no entanto, de uma equação integral válida não só para os pontos no domínio $\Omega$, como também para os pontos pertencentes ao contorno $\Gamma$. Esta expressão, aplicada a diferentes pontos no contorno, produz um sistema de equaçòes lineares que, uma vez resolvido, fornece os valores das variávcis no contorno.

No intuito de obter essa equação, far-se-á uma ampliaçào do domínio original $\Omega+\Gamma$, acrescentando-se, para isto, parte de uma esfera infinitesimal, denominado domínio $\Omega_{\varepsilon}$, de raio $\varepsilon$, centrado no ponto " $s$ ", conforme BREBBIA \& DOMINGUES (1984). Desta forma, o ponto " $s$ " do contorno passa a ser considerado como ponto do dominio, como mostra a Fig. 2.11.

Para o novo domínio $\Omega+\Omega_{\varepsilon}$ e contorno $\Gamma-\bar{\Gamma}+\Gamma_{\varepsilon}$, pode-se reescrever a identidade Somigliana, da seguinte forma: 


$$
\begin{aligned}
& u_{1}(S)=-\int_{\Gamma-\Gamma+\Gamma_{s}} P_{i j}^{*}(S, Q) u_{j}(Q) d \Gamma(Q)+\int_{\Gamma-\Gamma+\Gamma \varepsilon} u_{i j}^{*}(S, Q) p_{j}(Q) d \Gamma(Q)+ \\
& +\int_{\Omega+\Omega_{\varepsilon}} \mathbf{u}_{\mathrm{ij}}^{*}(\mathbf{s}, \mathbf{q}) \mathbf{b}_{\mathrm{j}}(\mathbf{q}) \mathrm{d} \Omega(\mathbf{q})
\end{aligned}
$$

Desmembrando-se esta expressão nos termos correspondentes ao domínio e ao contorno, tem-se:

$$
\begin{aligned}
u_{i}(S)= & -\int_{\Gamma-\Gamma} P_{i j}^{*}(S, Q) u_{i}(Q) d \Gamma(Q)-\int_{\Gamma_{\varepsilon}} P_{i j}^{*}(S, Q) u_{j}(Q) d \Gamma(Q)+ \\
& +\int_{\Gamma-\Gamma} U_{i j}^{*}(S, Q) p_{j}(Q) d \Gamma(Q)-\int_{\Gamma_{\varepsilon}} U_{i j}^{*}(S, Q) p_{j}(Q) d \Gamma(Q)+ \\
& +\int_{\Omega} U_{i j}^{*}(S, q) b_{j}(q) d \Omega(q)+\int_{\Omega_{\varepsilon}} U_{i j}^{*}(S, q) b_{j}(q) d \Omega(q)
\end{aligned}
$$

O próximo passo é analisar separadamente os limites dos seis termos da equação (2.31), com o objetivo de retornar ao domínio inicial, para o qual o ponto " $\mathrm{S}$ " pertencente ou externo ao contorno. Para isso, faz-se $\varepsilon \rightarrow 0$ e, consequentemente, $\Omega_{\varepsilon} \rightarrow 0$ e $\bar{\Gamma} \rightarrow 0$.
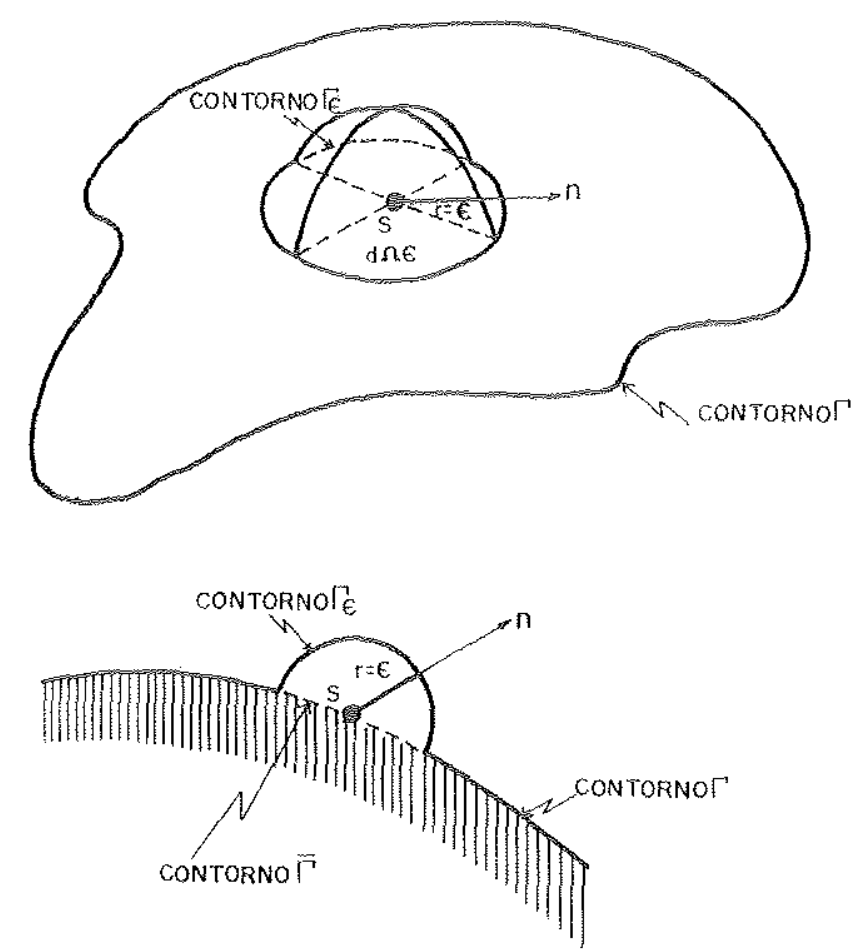

FIGURA 2.11 - Acréscimo ao domínio $\Omega$ de uma semi esfera infinitesimal de dominio $\Omega_{\varepsilon}$. 
Dessa forma, obtêm-sc os seguintes resultados para os limites das integrais no contorno $\Gamma-\bar{\Gamma}$ :

$\lim _{\Gamma \rightarrow 0}\left[\int_{\Gamma-\bar{\Gamma}} \mathbf{P}_{i j}^{*}(S, Q) \mathbf{u}_{j}(Q) d \Gamma(Q)\right]=\int_{\Gamma} \mathbf{P}_{i j}^{*}(S, Q) \mathbf{u}_{j}(Q) d \Gamma(Q)$

c,

$\lim _{\Gamma \rightarrow 0}\left[\int_{\Gamma-\Gamma} U_{i j}^{*}(S, Q) p_{j}(Q) d \Gamma(Q)\right]=\int_{\Gamma} U_{i j}^{*}(S, Q) p_{j}(Q) d \Gamma(Q)$

Em razão da descontinuidade que a função $\mathbf{P}_{\mathbf{i j}}{ }^{*}$ apresenta no limite, a integração da expressão (2.32) deve ser analisada no sentido de valor principal.

Considerando-se, agora, a quarta parcela, verifica-se que os valores de $\mathbf{U}_{\mathbf{i j}}$ * são da ordem de $\frac{1}{\varepsilon}$, enquanto a integração no contorno $\Gamma_{\varepsilon,}$ produz $\varepsilon^{2}$, podendo-se concluir que:

$$
\lim _{\varepsilon \rightarrow 0}\left[\int_{\Gamma_{\varepsilon}} U_{i j}^{*}(S, Q) p_{j}(Q) d \Gamma(Q)\right]=0
$$

Quanto às duas últimas parcelas, verifica-se que quando $\varepsilon \rightarrow 0$, a integral de $\Omega$ representa o domínio todo do problema, enquanto que $\Omega_{\varepsilon}$ tende a zero.

Resta, agora, analisar a segunda parcela de $(2.31)$, que se comporta de forma diferente. A integração dos valores do tensor $\boldsymbol{P}_{\mathbf{i j}}{ }^{*}$ no contorno $\Gamma_{\varepsilon}$ são da ordem de $\frac{1}{\varepsilon^{2}}$, enquanto os termos resultantes da integração sobre a superfícic são da ordem $\varepsilon^{2}$. Portanto, a integral não tem valor definido quando $\varepsilon \rightarrow \mathbf{0}$, mas produz um termo independente, que pode ser calculado pela substituição das equações $(2.21)(\mathrm{cm}$ termos de forças de superficic) e a consideração de que o ponto " $\mathrm{S}$ " pertence a um contorno sem angulosidade (dito "smooth"). Assim, pode-se escrever: 


$$
\begin{aligned}
& \lim _{\varepsilon \rightarrow 0}\left[\int_{\Gamma_{\varepsilon}} P_{i j}^{*}(S, Q) u_{j}(Q) d \Gamma(Q)\right]= \\
& =u_{j}(S) \lim _{\varepsilon \rightarrow 0}\left[\int_{\Gamma_{\varepsilon}} P_{i j}^{*}(S, Q) d \Gamma(Q)\right]=u_{j}(S)\left[-\frac{1}{2} \delta_{i j}\right]= \\
& =-\frac{1}{2} \delta_{i j} u_{j}(S)
\end{aligned}
$$

Deste modo, substituindo-se os resultados dos limites das integrais, na expressão $(2.31)$, tem-se:

$$
\begin{aligned}
C_{i j}(S) u_{j}(S)= & -\int_{\Gamma} P_{i j}^{*}(S, Q) u_{j}(Q) d \Gamma(Q)+\int_{\Gamma} U_{i j}^{*}(S, Q) p_{j}(Q) d \Gamma(Q)+ \\
& \left.+\int_{\Omega} U_{i j}^{*}(S, q)\right)_{j}(q) d \Omega(q)
\end{aligned}
$$

que é a expressão geral para pontos do domínio e para pontos do contorno, sendo que $\mathrm{C}_{\mathrm{ij}}(\mathrm{S})$ corresponde $\mathrm{a}$ :

$$
\mathrm{C}_{\mathrm{ij}}(\mathrm{S})=\delta_{\mathrm{ij}}-\frac{1}{2} \delta_{\mathrm{ij}}=\frac{1}{2} \delta_{\mathrm{ij}}
$$

Uma expressão integral para pontos fora do domínio pode ser escrita de forma similar a cq. (2.36), em que o primeiro termo da equação é nulo. Isto decorre da aplicação da terceira propriedade da distribuição delta de Dirac, ou seja:

$$
\int_{\Omega} \delta(s, q) u(q) d \Omega=u(s)=0
$$

Pode-se agora, conhecendo-se as expressòes integrais de deslocamentos para pontos internos e externos ao domínio e para pontos no contorno, afirmar que a eq. (2.36) é uma representação geral, desde que, admita-se, para o coeficiente $\mathbb{C}_{\mathrm{ij}}(\mathfrak{S})$, os seguintes valores:

$\delta_{i j} \rightarrow$ para pontos internos;

$$
\begin{aligned}
& \frac{\delta_{11}}{2} \rightarrow \text { para pontos no contomo sem angulosidade; } \\
& 0 \rightarrow \text { para pontos extcrnos. }
\end{aligned}
$$


Considerando a forma matricial, tem-se:

$$
\delta_{\mathrm{ij}}=\mathbf{I} \text {, }
$$

sendo que I é a matriz identidade de ordem 3.

Nos capítulos 3 e 4 , será apresentado um tratamento numérico para a resolução das equaçòes integrais de contorno, expressas em (2.36). 


\section{MÉTODO DOS ELEMENTOS DE CONTORNO PARA SÓLIDOS TRIDIMENSIONAIS}

\subsection{INTRODUÇÄO}

No capitulo anterior foram apresentadas as equaçòes integrais para um sólido tridimensional clástico linear isótropo. No entanto, tais equaçòes não permitem uma aplicação imediata na solução de problemas de engenharia, visto que são poucos $\mathrm{e}$ simples os problemas que apresentam soluçào exata. Assim, aproximaçòes devem ser introduzidas, de forma a permitir a solução do problema, reproduzindo da melhor forma possivel o problema real. Para tanto, o contorno do sólido é discretizado $\mathrm{cm}$ uma série de elementos de superfícic cuja geometria é aproximada em função de scus nós gcométricos, mediante funçòcs de interpolação. Em cada um desses elementos, os deslocamentos e forças de superficie são, também, aproximados $\mathrm{cm}$ relação aos seus valores nodais.

\subsection{DISCRETIZAÇÃO DO CONTORNO}

A discretizaçào do contorno de um sólido tridimensional é realizada dividindo-o em um número finito de elementos de superfície, que podem ser planos ou curvos (Fig. 3.1), denominados, também, elementos lineares ou quadráticos; os mais utilizados são de forma triangular e quadrilateral. 

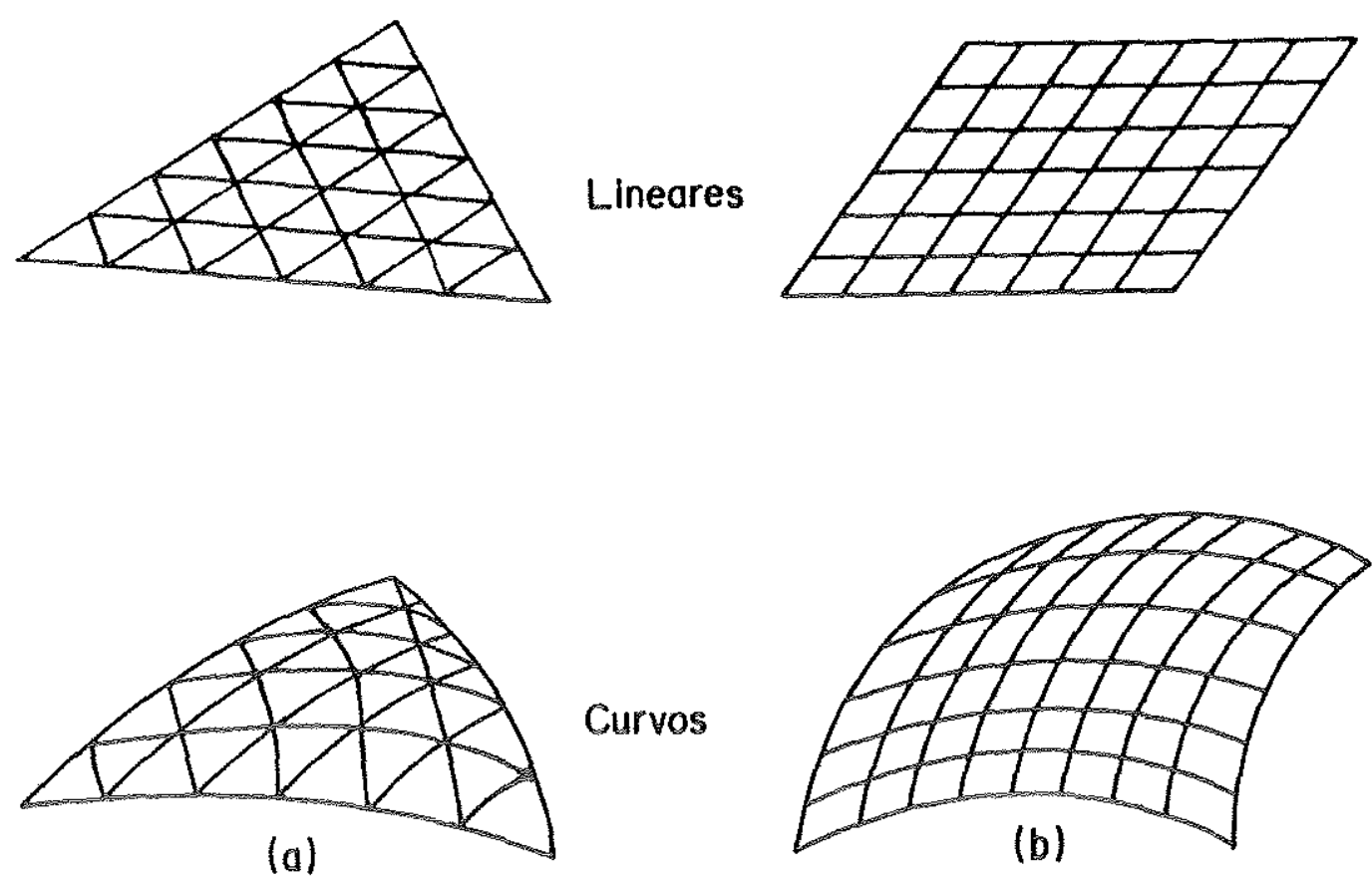

Curvos

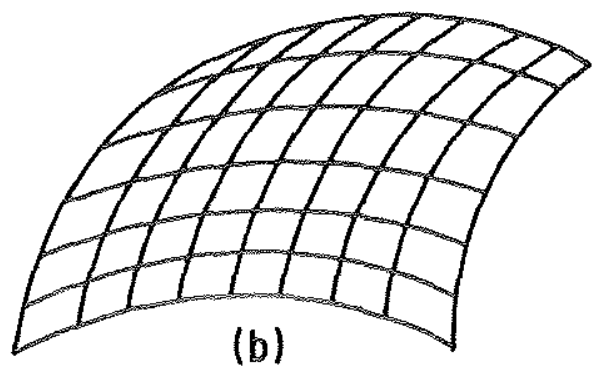

FIGURA 3.1. - Tipos de elementos utilizados para aproximar o contorno: a) triangulares; b) quadrilaterais.

Um elemento qualquer fica inteiramente definido pelas coordenadas de seus pontos nodais $\mathbb{X}_{i}^{k}$. As coordenadas cartesianas $\mathbb{X}_{\mathbf{i}}$ de um ponto $\mathbf{P}$ qualquer deste elemento săo expressas $\mathrm{cm}$ termos de funçôes interpoladoras $\Psi_{k}$ e de suas coordenadas nodais:

$$
X_{i}=\Psi_{k} X_{i}^{k}
$$

ou pela relaçào matricial:

$$
\mathbb{Z}=\Psi_{\sim}^{\mathbb{T}} \mathbb{X}^{\mathbf{N}}
$$

sendo que:

X : são as coordenadas cartesianas do ponto $\mathrm{P}$ :

$\mathbb{X}^{N}$ : são as coordenadas dos pontos nodais do clemento:

$\Psi_{\sim}^{T}$ : são as funçòes aproximadoras ou interpoladoras. 
As funções interpoladoras são, geralmente, polinomiais, previamente escolhidas, expressas em coordenadas adimensionais, que permitem, juntamente com as coordenadas cartesianas dos pontos nodais, a definição adequada da geometria do elemento.

O domínio do sólido também pode ser discretizado em elementos de volume, denominados células, geralmente, de forma tetraédrica, pentaćdrica e hexaédrica, como mostra a Fig. 3.2.

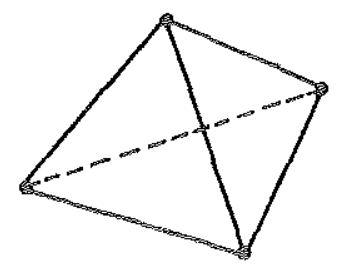

(a)

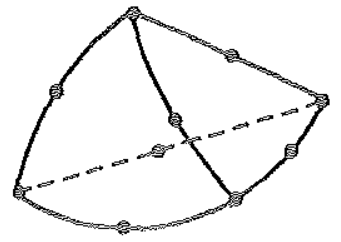

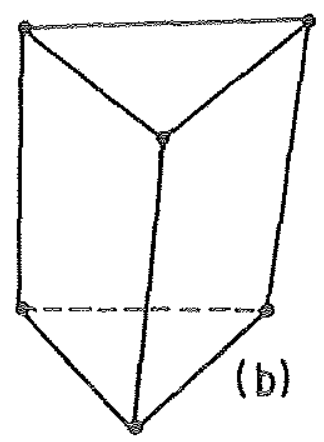

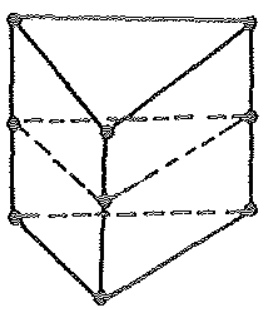

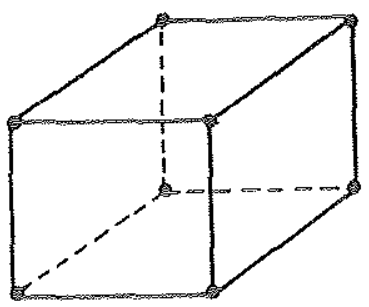

(c)

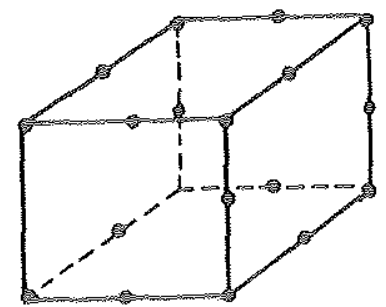

FIGURA 3.2 - Tipos de células para aproximar o domínio: a) tetraćdricas; b) pentaédricas; c) hexaédricas.

Funçòes interpoladoras $\Psi_{\mathrm{c}}$ são usadas para exprimir as coordenadas cartesianas $\underset{\sim}{\mathbf{X}}$ dos pontos em cada célula, em termos das coordenadas nodais $\underset{\sim}{\mathbb{N}}$ da célula, isto é:

$$
\underset{\sim}{\mathbb{X}}=\underset{\sim \mathrm{c}}{\Psi_{\sim}^{\mathrm{T}}} \underset{\mathrm{c}}{\mathbb{X}}
$$

De maneira similar, sobre cada clemento, são também aproximadas as variáveis envolvidas no problema, neste caso, deslocamentos e forças de superficic, $\mathrm{em}$ função de seus valores nos nós funcionais, empregando-se funções polinomiais. 
Tais funções podem ser constantes ou com variação linear, quadrática ou de ordem superior, como mostra a Fig. 3.3 .
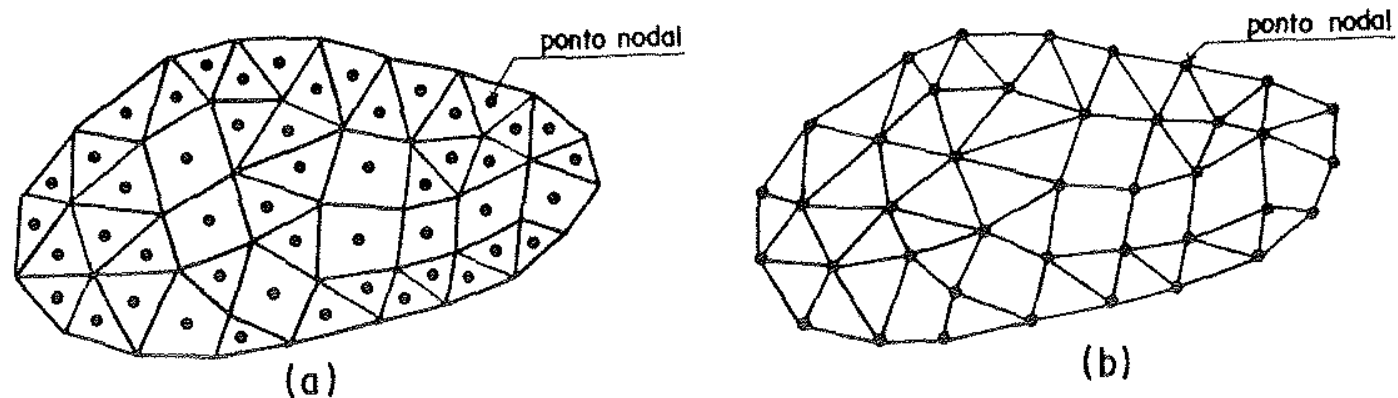

(b)
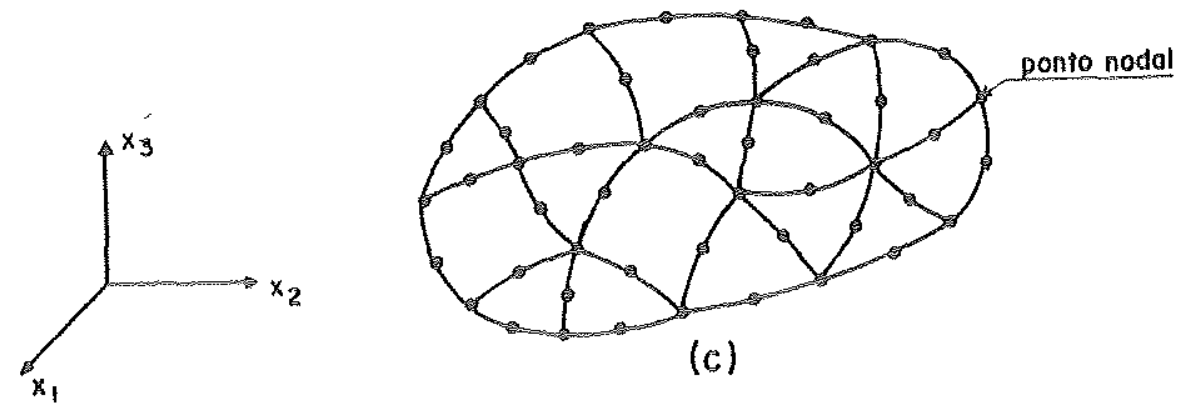

FIGURA 3.3 - Discretização do contomo de um corpo em clementos: a) constante; b) linear ; c) quadrático.

Da mesma forma que se aproximou a geometria, os deslocamentos u e forças de superficie podem também ser aproximados por meio de funções interpoladoras $\Phi$ e valores nodais $U^{N}$ e $P^{N}$, respectivamente, como:

$$
\begin{aligned}
& \underline{u}=\boldsymbol{\Phi}_{\sim}^{\mathrm{Y}} \mathbf{U}^{\mathrm{N}} \\
& p=\overline{\mathbb{D}}^{\mathrm{T}} \mathrm{P}^{\mathrm{N}}
\end{aligned}
$$

As forças volumétricas $\mathbf{b}$, de forma análoga às variáveis de contorno, podem ser aproximadas sobre cada célula por funçòes interpoladoras $\underset{\sim}{\Phi}$ e valores nodais $\mathbb{B}^{N}$ 


$$
\underset{\sim}{\mathbf{b}}=\underset{\sim}{\Phi_{\mathfrak{c}}^{\mathrm{T}}}{\underset{\sim}{\mathbf{B}}}^{\mathrm{N}}
$$

Após expor a forma como a geometria e as variáveis envolvidas podem ser aproximadas, retomar-se-á a equaçăo integral de deslocamentos $(2.36) \mathrm{cm}$ sua forma matricial, escrita como:

$$
\begin{aligned}
& \mathrm{C}(\mathrm{S}) \underset{\sim}{\mathbf{u}}(\mathrm{S})+\int_{\Gamma \sim}^{\mathbf{p}}{ }_{\sim}^{*}(\mathrm{~S}, \mathrm{Q}) \underset{\sim}{\mathbf{u}}(\mathrm{Q}) \mathrm{d} \Gamma(\mathrm{Q})=\int_{\Gamma} \underset{\sim}{\mathbf{u}^{*}}(\mathrm{~S}, \mathrm{Q}) \underset{\sim}{\mathbf{p}}(\mathrm{Q}) \mathrm{d} \Gamma(\mathrm{Q}) \\
& +\int_{\Gamma} u_{\sim}^{*}(S, q) \underset{\sim}{b}(q) d \Omega(q)
\end{aligned}
$$

Discretizando-se o contorno do sólido em "J" clementos e seu domínio $\mathrm{cm}$ "M" células, a equação integral para um nó $\mathbf{S}$ qualquer do contorno, torna-se:

$$
\begin{aligned}
& \underset{\sim}{C}(S) \underset{\sim}{\mathbf{u}}(S)+\sum_{j=1}^{\mathrm{J}}\left[\int_{\Gamma_{j} \sim} \mathbf{p}^{*}(S, Q) \underset{\sim}{\Phi}{ }^{\mathrm{T}}(Q) d \Gamma(Q)\right] \underset{\sim}{U^{N}}(\mathbb{Q})= \\
& =\sum_{j=1}^{J}\left[\int_{\Gamma_{j}} u^{*}(S, Q) \underset{\sim}{\Phi^{T}}(Q) d \Gamma(Q)\right] P^{N}(Q)+ \\
& +\sum_{m=1}^{\mathrm{n}}\left[\int_{\Omega_{\mathrm{m}}} \underline{\sim}^{*}(\mathcal{S}, q) \underset{\sim \mathrm{c}}{\boldsymbol{\Phi}^{\mathrm{T}}}(q) \mathrm{d} \Gamma(q)\right] \mathrm{B}_{\sim}^{\mathrm{N}}(q)
\end{aligned}
$$

Aplicando esta cquação aos "N" pontos nodais do contomo do sólido, estabelece-se um sistema de equaçóes que, escrito em forma matricial, é dado por:

$$
\underset{\sim}{\mathrm{CU}}+\underset{\sim}{\tilde{H}} \mathbf{U}=\underset{\sim}{\mathrm{G}} \mathrm{P}+\underset{\sim}{\mathrm{D}} \mathrm{B}
$$

sendo que as matrizes $\hat{H}, G$ e $D$ resultam do cálculo das integrais da equação (3.8), as quais serão tratadas posteriormente.

$$
\begin{aligned}
& \text { As matrizes } \underset{\sim}{\mathbb{C} e \hat{H}} \text { da equação (3.9) são somadas, resultando: } \\
& \underset{\sim}{\mathbf{H}}=\underset{\sim}{\mathbf{G}} \mathbf{P}+\underset{\sim}{\mathbf{D}}
\end{aligned}
$$

Conhecidos os valores prescritos dos deslocamentos $\underset{\sim}{U}$ das forças de superficic $\underset{\sim}{\mathbf{P}}$ e das forças volumétricas $\underset{\sim}{\mathbf{B}}$, as colunas das matrizes $\prod_{\sim}$ e $\mathbf{G}$ na equação (3.10) são trocadas entre si, de maneira que todas as incógnitas fíquem no 
primeiro membro; obtém-se, assim, um sistema de equações algébricas com "3N" incógnitas, do tipo:

$$
\underset{\sim}{\mathbf{B}} \mathbf{X}=\underset{\sim}{\mathbf{F}}
$$

Neste sistema, $\mathbf{X}$ é o vetor das incógnitas, constituido por deslocamentos e forças de superficic, $\mathbf{B}$ è a matriz cheia e não simćtrica composta por colunas das matrizes $\underset{\sim}{\mathbf{H}}$ c $\mathbf{G}, \mathrm{c} \underset{\sim}{\mathbf{F}}$ é o vetor formado pela multiplicaçào dos valores prescritos ( ${ }_{\sim}$ e $\mathbf{P}$ ), com as correspondentes colunas de $\underset{\sim}{\mathbf{H}} \mathrm{e} \mathbf{G}$, mais a contribuição das forças volumétricas.

A solução do sistema de equaçoes (3.11) pode ser obtida, por cxemplo, como:

$$
\underset{\sim}{X}=\mathbb{B}_{\sim}^{-1} \mathbf{F}
$$

onde $\mathbb{B}_{\sim}^{-1}$ é a matriz inversa de $\underset{\sim}{\mathbb{B}}$.

\subsection{GEOMETRIA DOS ELEMENTOS}

Como já foi dito, o contorno de sólidos tridimensionais pode ser discretizado por clcmentos planos ou curvos, tendo a forma triangular ou quadrilateral. Neste trabalho, serão utilizados os clementos triangulares planos para discretizar o contorno dos sólidos.

O elemento triangular linear fica definido se forem conhecidas as coordenadas cartesianas de seus pontos nodais localizados em seus vértices (Fig. 3.4) e as funções aproximadoras, expressas $\mathrm{cm}$ coordenadas naturais (triangulares) ou homogêneas. 


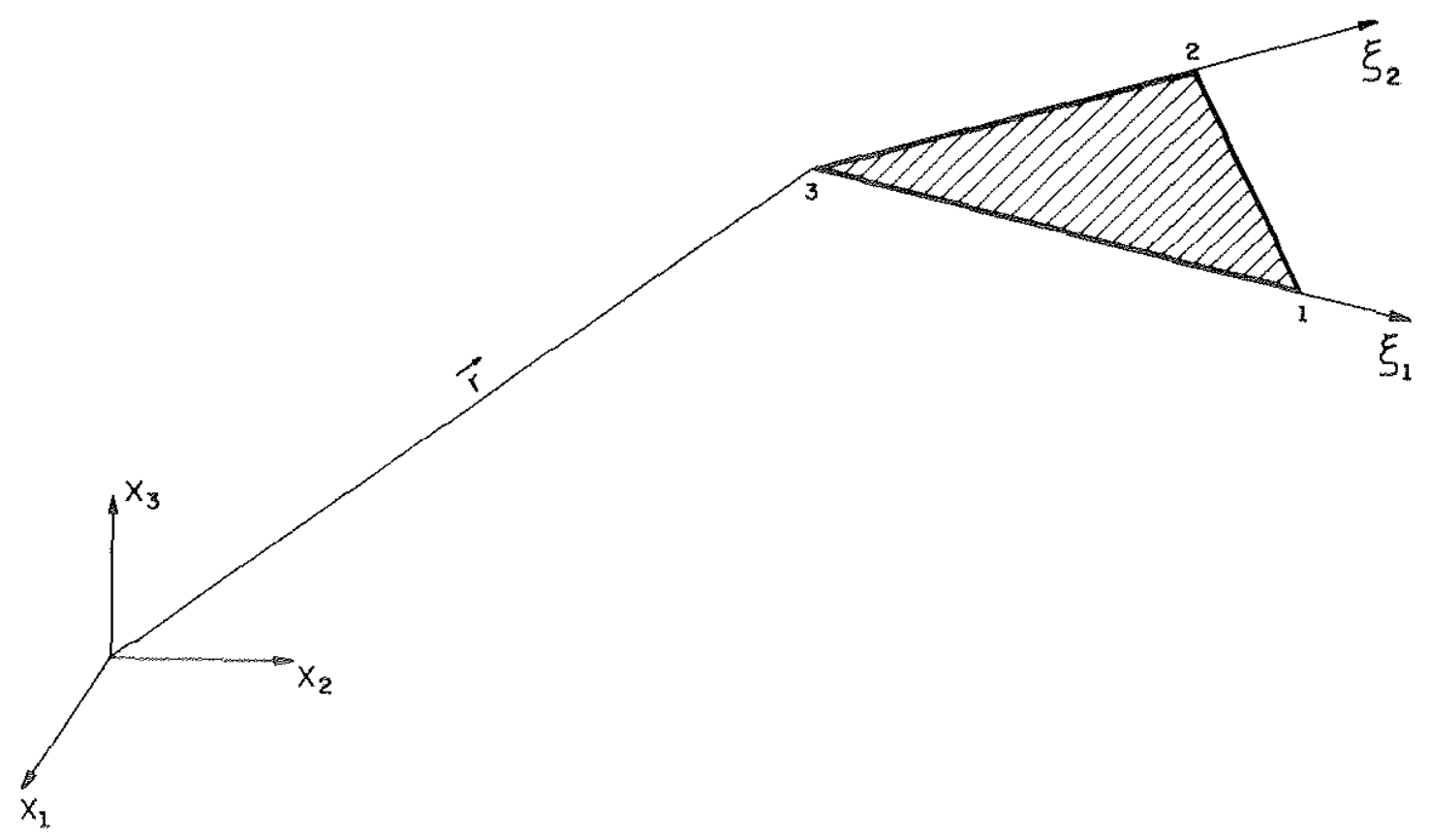

FIGURA 3.4 - Coordenadas globais e naturais (locais) para o elemento triangular plano.

As coordenadas homogèneas ou triangulares facilitam a representação de funções de interpolação c simplificam integrações quando se opera com os métodos dos elementos de contorno ou dos elementos finitos, com elementos triangulares.

Examinar-se-á o elemento triangular mostrado na Fig. 3.5. Admita-se um sistema de coordenadas cartesianas local, cujos cixos y 1 e y2 estão contidos no plano do elemento. Pode-se definir a posição de um ponto $\mathbf{P}$ qualquer do triângulo pelas suas coordenadas $(y 1, y 2)$, que, juntamente com os vértices do triângulo, definem as areas $A_{1}, A_{2}$ e $A_{3}$, tais que:

$$
\mathbf{A}=\mathbb{A}_{1}+\mathbf{A}_{2}+\mathbb{A}_{3}
$$

sendo $\mathrm{A}$ a área total do triàngulo de vértices $1,2,3$. Uma vez que os valores de $A_{1}$, $A_{2}$ e $A_{3}$ são únicos para cada ponto pertencente ao triângulo, pode-se concluir que estes podem ser usados para definir a posição de pontos no triàngulo, isto ć, como coordenadas. Na prática, contudo, é melhor trabalhar com valores adimensionais. As coordenadas homogêneas ou triangulares săo, então, definidas como: 


$$
\begin{aligned}
& \xi_{1}=\frac{\mathbf{A}_{1}}{\mathbf{A}} \\
& \xi_{2}=\frac{\mathbf{A}_{2}}{\mathbf{A}} \\
& \xi_{3}=\frac{\mathbf{A}_{3}}{\mathbf{A}}
\end{aligned}
$$

Note que, a partir das equaçòes $(3.13),(3.14),(3.15)$ e $(3.16)$, podemos verificar que:

$$
\xi_{1}+\xi_{2}+\xi_{3}=1
$$

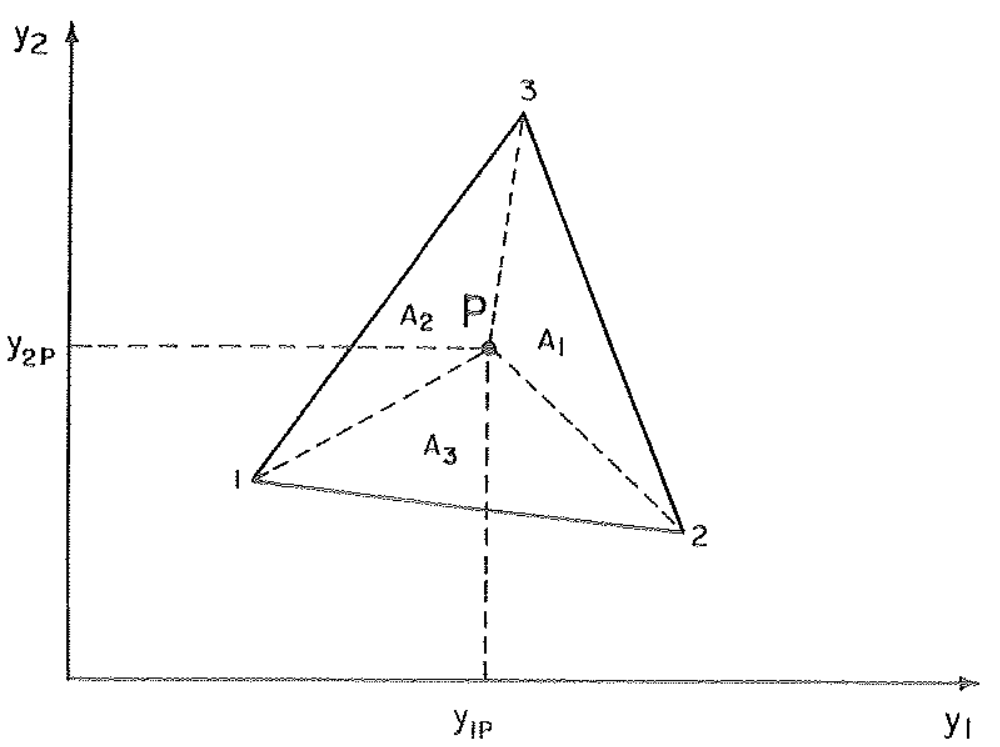

FIGURA 3.5 - Coordenadas cartesianas do ponto P.

Da equação (3.17), pode-se concluir que nem todas as três coordenadas triangulares são independentes. A Fig. 3.6 mostra os valores das coordenadas homogêneas $\left(\xi_{1}, \xi_{2}, \xi_{3}\right)$ para alguns pontos relevantes, cujos valores variam entre 0 c 1. 


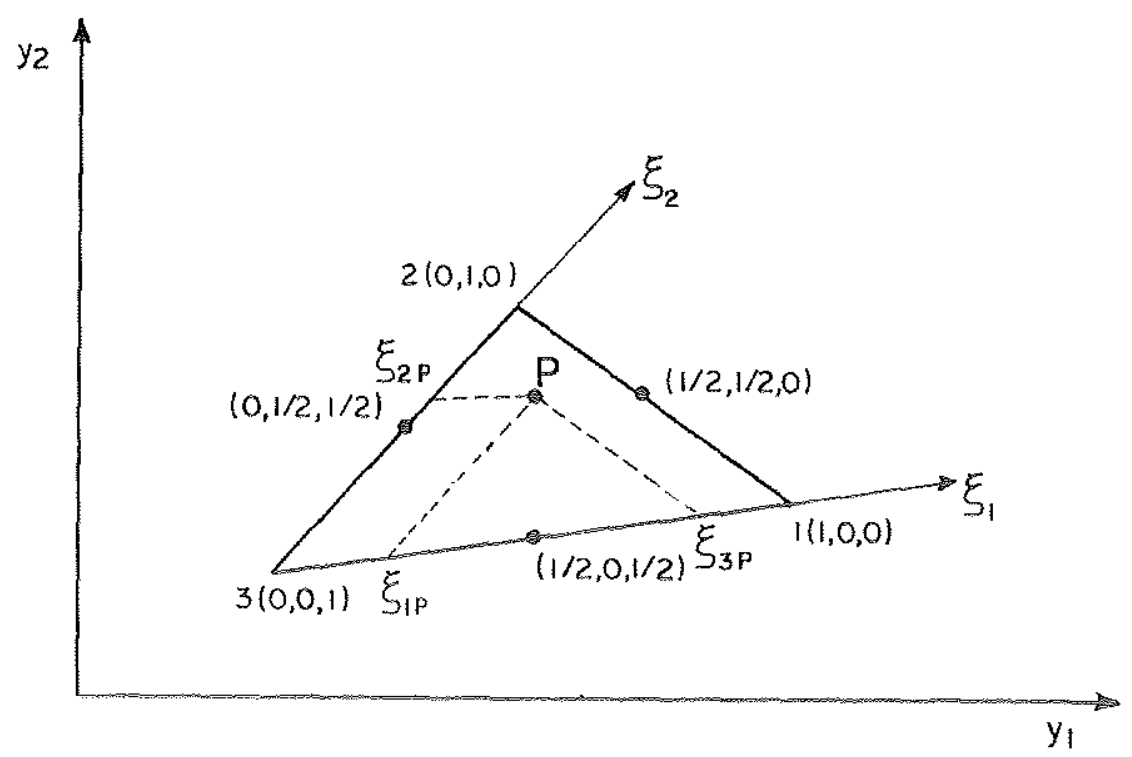

FIGURA 3.6 - Coordenadas naturais para alguns pontos do triângulo.

Pode-se estabelecer relações cntre as coordenadas triangulares e cartesianas, apresentando as expressòes para as áreas $A_{1}, A_{2}$ e $A_{3}$ em termos das coordenadas dos vértices do elemento triangular, como:

$$
\begin{aligned}
& \mathbb{A}_{1}=\frac{1}{2}\left|\begin{array}{lll}
y_{1} & y_{2} & 1 \\
y_{1}^{2} & y_{2}^{2} & 1 \\
y_{1}^{3} & y_{2}^{3} & 1
\end{array}\right| \\
& \mathbb{A}_{2}=\frac{1}{2}\left|\begin{array}{lll}
y_{1}^{1} & y_{2}^{1} & 1 \\
y_{1} & y_{2} & 1 \\
y_{1}^{3} & y_{2}^{3} & 1
\end{array}\right| \\
& \mathbb{A}_{3}=\frac{1}{2}\left|\begin{array}{lll}
y_{1}^{1} & y_{2}^{1} & 1 \\
y_{1}^{2} & y_{2}^{2} & 1 \\
y_{1} & y_{2} & 1
\end{array}\right|
\end{aligned}
$$


Nestas equações, para o sistema de coordenadas local, $\mathbf{y}_{\mathbf{1}}^{\mathbf{j}}$, "ij" corresponde as direções 1, 2 e "j" o número dos vértices do elemento triangular $(1,2$ e 3$)$.

Tendo em vista a definição de coordenadas homogeneas, verifica-se que:

$$
\begin{aligned}
& \frac{A_{1}}{A}=\xi_{1}=\frac{1}{2 A}\left[y_{1}\left(y_{2}^{2}-y_{2}^{3}\right)+y_{2}\left(y_{1}^{3}-y_{1}^{2}\right)+y_{1}^{2} y_{2}^{3}-y_{1}^{3} y_{2}^{2}\right] \\
& \frac{A_{2}}{A}=\xi_{2}=\frac{1}{2 A}\left[y_{1}\left(y_{2}^{3}-y_{2}^{1}\right)+y_{2}\left(y_{1}^{1}-y_{1}^{3}\right)+y_{1}^{3} y_{2}^{1}-y_{1}^{1} y_{2}^{3}\right] \\
& \frac{A_{3}}{A}=\xi_{3}=\frac{1}{2 A}\left[y_{1}\left(y_{2}^{1}-y_{2}^{2}\right)+y_{2}\left(y_{1}^{2}-y_{1}^{1}\right)+y_{1}^{1} y_{2}^{2}-y_{1}^{2} y_{2}^{1}\right]
\end{aligned}
$$

Assim, podemos escrever uma expressão geral, como:

$$
\xi_{i}=a_{i} y_{1}+b_{i} y_{2}+c_{i} \quad(i=1,2,3)
$$

sendo que:

$$
\begin{array}{ll}
a_{i}=\frac{1}{2 A}\left(y_{2}^{j}-y_{2}^{k}\right) & (i=1,2,3) \\
b_{i}=\frac{1}{2 A}\left(y_{1}^{k}-y_{1}^{j}\right) & (j=2,3,1) \\
c_{1}=\frac{1}{2 A}\left(y_{1}^{j} y_{2}^{k}-y_{1}^{k} y_{2}^{j}\right) & (k=3,1,2)
\end{array}
$$

onde, $\mathrm{i}, \mathrm{j}$ e $\mathrm{k}$ sào tomados em seqüencia e ciclicamente, como indicado acima.

A equação $(3.24)$, apresentada matricialmente, toma a forma:

$$
\left\{\begin{array}{c}
\xi_{1} \\
\xi_{2} \\
\xi_{3}
\end{array}\right\}=\left[\begin{array}{lll}
a_{1} & b_{1} & c_{1} \\
a_{2} & b_{2} & c_{2} \\
a_{3} & b_{3} & c_{3}
\end{array}\right]\left\{\begin{array}{c}
y_{1} \\
y_{2} \\
1
\end{array}\right\},
$$

ou, ainda:

$$
\xi=\mathbb{C} \mathbf{y}
$$

Invertendo a equação (3.28), obtem-se a relaçâo entre as coordenadas cartesianas e triangulares, expressas como: 


$$
\left\{\begin{array}{c}
y_{1} \\
y_{2} \\
1
\end{array}\right\}=\left[\begin{array}{ccc}
y_{1}^{1} & y_{1}^{2} & y_{1}^{3} \\
y_{2}^{1} & y_{2}^{2} & y_{2}^{3} \\
1 & 1 & 1
\end{array}\right]\left\{\begin{array}{l}
\xi_{1} \\
\xi_{2} \\
\xi_{3}
\end{array}\right\}
$$

Retornemos, agora, à Fig. 3.4; a partir da equação (3.2), as coordenadas cartesianas de um ponto qualquer pertencente ao clemento triangular são dadas por:

$$
\underset{\sim}{X}=\Psi^{\mathrm{T}}(\xi) \underset{\sim}{\mathbf{X}^{N}}
$$

onde:

$$
\begin{aligned}
& x=\left\{\begin{array}{l}
x_{1} \\
x_{2} \\
x_{3}
\end{array}\right\} \\
& \Psi^{\mathrm{T}}=\left[\begin{array}{ccc}
\psi^{\mathrm{T}} & 0 & 0 \\
\sim & \Psi^{\mathrm{T}} & 0 \\
\sim & \sim & \sim \\
0 & 0 & \Psi^{\mathrm{T}} \\
\sim & \sim & \sim
\end{array}\right] \\
& \mathbb{X}^{\mathrm{N}}=\left\{\begin{array}{c}
\mathbb{X}^{\mathrm{N}} \\
\sim 1 \\
\mathbb{X}^{N} \\
\sim 2 \\
\mathbb{X}^{N} \\
\sim 3
\end{array}\right\}
\end{aligned}
$$

A função interpoladora $\psi$, cxibida na equação (3.33), pode ser expressa em coordenadas homogèneas por:

$$
\underset{\sim}{\Psi}=\left\{\begin{array}{l}
\xi_{1} \\
\xi_{2} \\
\xi_{3}
\end{array}\right\}
$$

As coordenadas cartesianas dos três nós dos vértices do clemento triangular podem ser representadas por: 


$$
\underset{\sim i}{X^{N}}=\left\{\begin{array}{l}
X_{1}^{1} \\
X_{1}^{2} \\
X_{i}^{3}
\end{array}\right\}(i=1,2,3)
$$

sendo "N" o número do nó c "i", a direção.

Explicitando-se, a equação (3.31) toma a forma:

$$
\left\{\begin{array}{l}
x_{1} \\
x_{2} \\
x_{3}
\end{array}\right\}=\left[\begin{array}{ccccccccc}
\xi_{1} & 0 & 0 & \xi_{2} & 0 & 0 & \xi_{3} & 0 & 0 \\
0 & \xi_{1} & 0 & 0 & \xi_{2} & 0 & 0 & \xi_{3} & 0 \\
0 & 0 & \xi_{1} & 0 & 0 & \xi_{2} & 0 & 0 & \xi_{3}
\end{array}\right]\left\{\begin{array}{l}
X_{1}^{1} \\
X_{2}^{1} \\
X_{3}^{1} \\
X_{1}^{2} \\
X_{2}^{2} \\
X_{3}^{2} \\
X_{1}^{3} \\
X_{2}^{3} \\
X_{3}^{3}
\end{array}\right\}
$$

Para o emprego de coordenadas triangulares, as equaçoes integrais escritas originalmente $\mathrm{cm}$ um sistema de coordenadas cartesianas global, devem passar a ser expressas em coordenadas homogêneas, já que estas sào utilizadas na aproximação da geometria dos elementos, bem como das variáveis envolvidas. Assim, a seguinte transformação, válida para qualquer função $r$, deve ser efetuada:

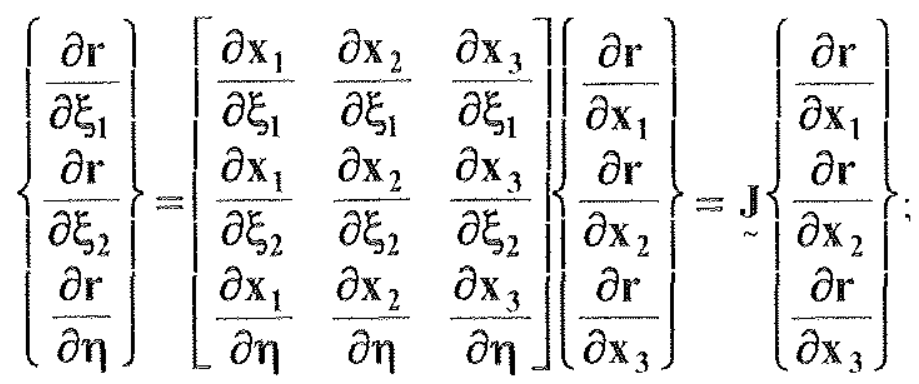

aqui, I é o Jacobiano que relaciona a diferenciação de coordenadas homogêneas para coordenadas cartesianas e $\eta$ é o versor normal ao plano do elemento.

Na Fig. 3.7, cstão indicadas as diferenciais do vetor posição $\mathrm{r} \mathrm{cm}$ relação às coordenadas homogèneas. 


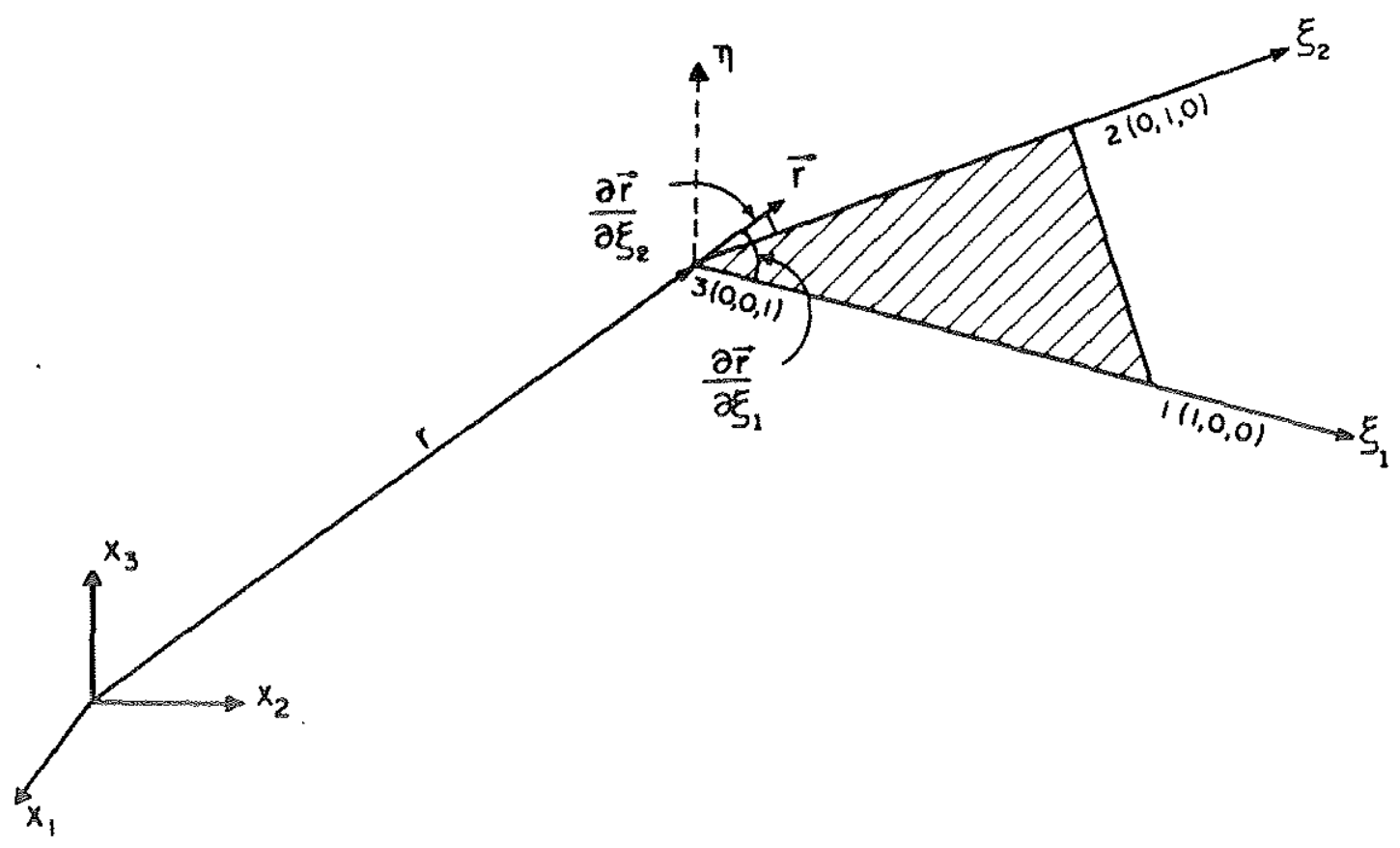

FIGURA 3.7 - Indicaçào dos sistemas de coordenadas.

A partir da equação (3.38), pode-se, agora, escrever a diferencial de volume:

$$
\mathbf{d} \Omega=\left|\frac{\partial \mathrm{r}^{*}}{\partial \xi_{1}} \times \frac{\partial \mathrm{r}}{\partial \xi_{2}} \cdot \frac{\partial \mathrm{r}^{\mathrm{b}}}{\partial \eta}\right| \mathrm{d} \xi_{1} \mathrm{~d} \xi_{2} \mathrm{~d} \eta=\left|J_{\sim}\right| \mathbf{d} \xi_{1} \mathrm{~d} \xi_{2} \mathrm{~d} \eta
$$

A diferencial de área do contorno $\mathbf{d} \Gamma$ pode ser representada como:

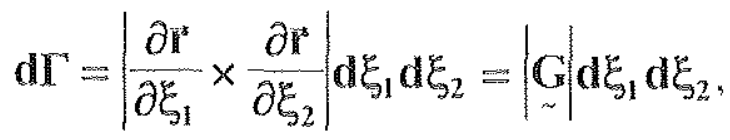

onde:

$$
\begin{aligned}
& \frac{\partial \vec{r}}{\partial \xi_{1}}=\left(\frac{\partial \mathbf{x}_{1}}{\partial \xi_{1}}, \frac{\partial \mathrm{x}_{2}}{\partial \xi_{1}}, \frac{\partial \mathrm{x}_{3}}{\partial \xi_{1}}\right) \\
& \frac{\partial \mathrm{r}}{\partial \xi_{2}}=\left(\frac{\partial \mathrm{x}_{1}}{\partial \xi_{2}}, \frac{\partial \mathrm{x}_{2}}{\partial \xi_{2}}, \frac{\partial \mathrm{x}_{3}}{\partial \xi_{2}}\right) \\
& |\underset{\sim}{\mathrm{G}}|=2 \mathrm{~A}
\end{aligned}
$$

Note que $|\underset{\sim}{\text { G }}|$ ć igual ao módulo do vetor normal ao elemento, que, no caso de clemento triangular plano, é numericamente igual ao dobro de sua árca "A". 
O vetor normal é dado pelo produto vetorial:

$$
\eta=\frac{\partial \overrightarrow{\mathbf{r}}}{\partial \xi_{1}} \times \frac{\partial \overrightarrow{\mathbf{r}}}{\partial \xi_{2}}
$$

Suas componentes em relaçào ao sistema de coordenadas global, ficam definidas como:

$$
\eta=\left(\frac{\partial \mathbf{x}_{1}}{\partial \eta}, \frac{\partial \mathbf{x}_{2}}{\partial \eta}, \frac{\partial \mathbf{x}_{3}}{\partial \eta}\right)
$$

Escrevendo a equação (3.31) na forma indicial, tem-se:

$$
x_{i}=\xi_{k} X_{i}^{k}
$$

Diferenciando a equação $(3.46)$ em relação às coordenadas homogêneas $\xi_{1}$ e $\xi_{2}$ c considerando-se a equação (3.17), na qual se admite $\xi_{3}$ como variável dependente, obtém-se:

$$
\begin{array}{ll}
\frac{\partial X_{i}}{\partial \xi_{1}}=X_{i}^{1}-X_{i}^{3} & \\
\frac{\partial X_{i}}{\partial \xi_{2}}=X_{i}^{2}-X_{i}^{3} &
\end{array}
$$

\subsection{FUNÇÕES DE INTERPOLAÇÄO}

As funções de interpolação das variàveis cnvolvidas nas equações integrais para sólidos tridimensionais. deslocamentos $\mathrm{e}$ forças de superfície, podem ser aproximadas de forma análogas às da geometria do elemento e dependem apenas da posição dos pontos nodais no elemento.

As funçòes de interpolaçào podem ser constantes, lincares, quadraticas ou de ordem superior. A scguir, serào apresentadas apcnas as funções de interpolação constante e lincar, utilizadas em elementos triangulares. 


\subsubsection{ELEMENTO TRIANGULAR CONSTANTE}

O clemento constante considera apenas um ponto nodal localizado. geralmente, $\mathrm{em}$ seu centróide. sendo que as variáveis são aproximadas pela função Ф. como mostra a Fig. 3.8.

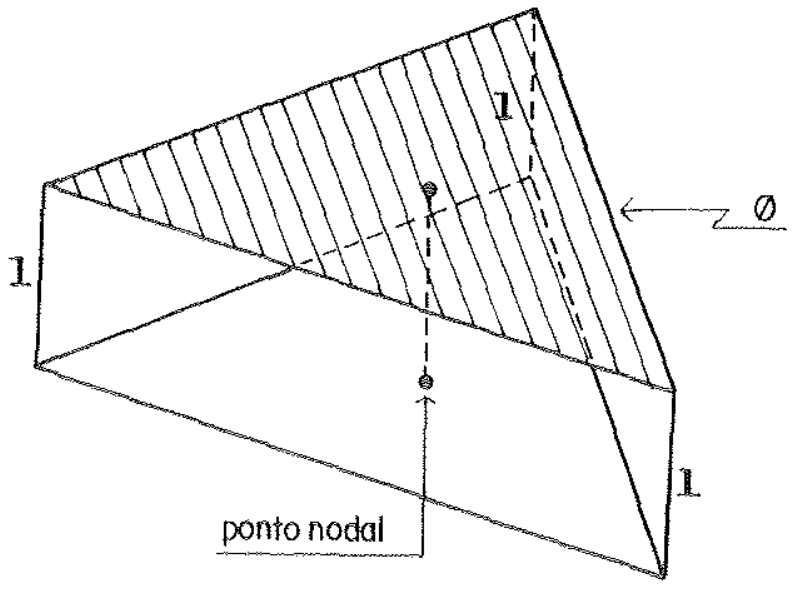

FIGURA 3.8- Elemento triangular constante.

O clemento triangular constante presta-se para aplicaçōes $\mathrm{cm}$ problemas com qualquer lipo de descontinuidade, sem nenhum problema de singularidade. apresentando bons resultados como os obtidos por LACHAT (1975), NAKAGUMA (1979) e SILVA (1989). Uma desvantagem desses elementos é a necessidade de um grande número de elementos para se obter resultados mais precisos.

Os deslocamentos e forças de superfícic são dadas por:

$$
\begin{aligned}
& \mathbf{u}=\mathbb{\Phi}^{\mathrm{T}} \mathbb{U}^{\mathrm{N}} \\
& \mathbf{p}=\overline{\boldsymbol{\Phi}}^{\mathrm{T}} \mathbf{P}^{\mathrm{N}}
\end{aligned}
$$

sendo que:

$$
u=\left\{\begin{array}{l}
u_{1} \\
u_{2} \\
u_{3}
\end{array}\right\} \quad: p=\left\{\begin{array}{l}
p_{1} \\
p_{2} \\
p_{3}
\end{array}\right\}
$$




$$
\begin{aligned}
& {\underset{\sim}{\Phi}}^{\mathrm{T}}=\left[\begin{array}{lll}
1 & 0 & 0 \\
0 & 1 & 0 \\
0 & 0 & 1
\end{array}\right]=\underline{\sim} \\
& {\underset{\sim}{U}}^{N}=\left\{\begin{array}{l}
U_{1}^{1} \\
U_{2}^{1} \\
U_{3}^{1}
\end{array}\right\} ; \quad \stackrel{\sim}{P}^{N}=\left\{\begin{array}{l}
P_{1}^{1} \\
P_{2}^{1} \\
P_{3}^{1}
\end{array}\right\}
\end{aligned}
$$

$\mathrm{O}$ indice $\mathrm{N} \dot{e}$ utilizado para indicar os valores dos deslocamentos e forças de superticic nos pontos nodais do clemento. Assim, $\mathbf{U}_{\mathbf{i}}^{N}$ \& $\mathbf{P}_{\mathbf{i}}^{\mathbf{N}}$, representam respectivamente o deslocamento e força de superficie na direção i do ponto nodal $\mathbf{N}$.

\subsubsection{ELEMENTO TRIAVGULAR LIVEAR}

O clemento triangular linear conta com tress pontos nodais. os quais permitem uma melhor aproximação das variáveis do que o elemento constante. Entretanto, o clemento lincar apresenta problemas de descontinuidade. especialmente em relação às forças de superficic. No que se refere à continuidade, o elemento pode ser classilicado como contínuo de transição e descontínuo (Fig. 3.9).
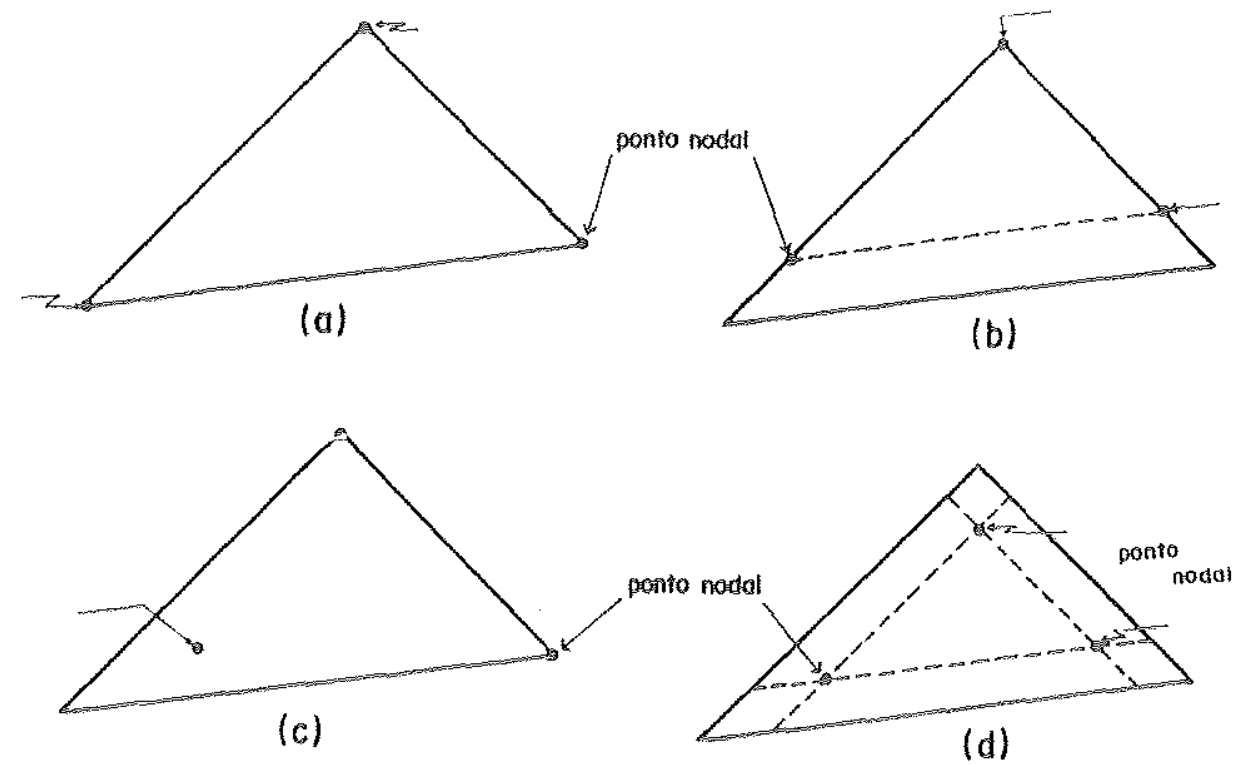

FIGURA 3.9 - Elementos triangulares lincares: a) contínuo; b) e c) de transição: d) descontínuo. 


\subsubsection{ELEMENTO TRIANGULAR LINEAR CONTÍNUO}

O elemento triangular linear contínuo é aquele $\mathrm{cm}$ que os pontos nodais coincidem com os nós geométricos localizados nos vértices 1,2 e 3 do elemento, onde as variáveis são aproximadas por lunçōes de interpolação $\phi^{1}, \phi^{2}$ e $\phi^{3}$, respectivamente. como mostra a Fig. 3.10 .

Cada função de interpolação $\phi^{i}$ tem valor unitário no ponto nodal i e zero nos demais pontos nodais; assim. essas funções podem ser representadas por:

$$
\begin{aligned}
\phi^{\prime} & =\xi_{1} \\
\phi^{2} & =\xi_{2}, \\
\phi^{3} & =\xi_{3}
\end{aligned}
$$

sendo, $\xi^{1}, \xi^{2} \mathrm{c} \xi^{3}$ as coordenadas homogeneas.
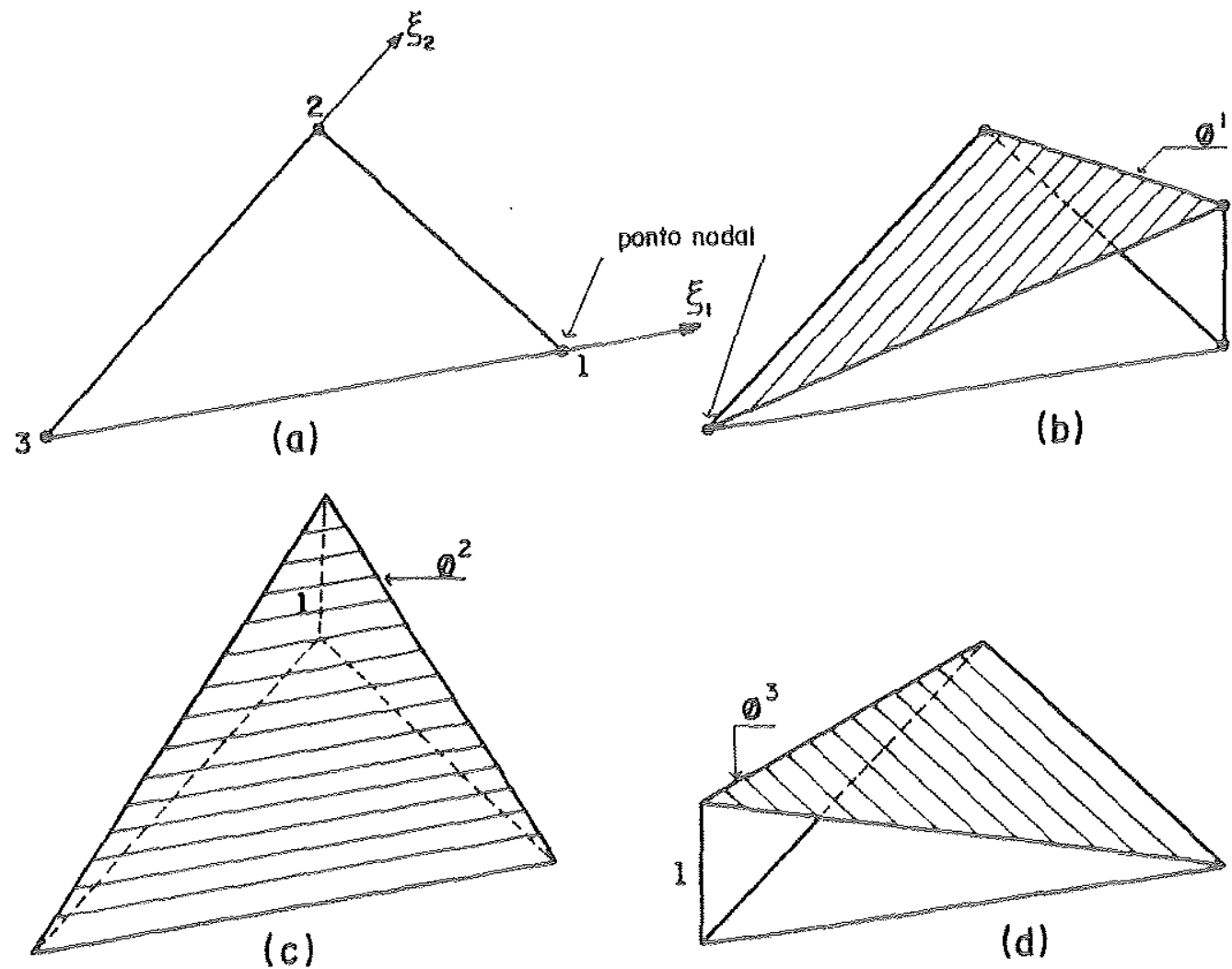

FIGURA 3.10 - Funções interpoladoras para o elemento triangular linear contínuo. 
Estc clemento não possibilita a inclusão de descontinuidades; por esta razão, cle só poderá ser utilizado $\mathrm{cm}$ contornos onde não existam angulosidades.

Para o clemento ora apresentado, os deslocamentos e forças de superficie são dados por:

$$
\begin{aligned}
& \mathbf{u}=\Phi^{\mathrm{T}}(\xi) \mathbf{U}^{\mathbf{N}} \\
& \mathbf{p}=\boldsymbol{\Phi}^{\mathrm{T}}(\xi) \mathbf{P}_{\sim}^{\mathrm{N}}
\end{aligned}
$$

onde:

$$
\begin{aligned}
& \mathbf{u}=\left\{\begin{array}{l}
u_{1} \\
u_{2} \\
u_{3}
\end{array}\right\}: p=\left\{\begin{array}{l}
p_{1} \\
p_{2} \\
p_{3}
\end{array}\right\} \\
& \underset{\sim}{\Phi^{\top}}=\left[\begin{array}{ccccccccc}
\phi^{1} & 0 & 0 & \phi^{2} & 0 & 0 & \phi^{3} & 0 & 0 \\
0 & \phi^{1} & 0 & 0 & \phi^{2} & 0 & 0 & \phi^{3} & 0 \\
0 & 0 & \phi^{1} & 0 & 0 & \phi^{2} & 0 & 0 & \phi^{3}
\end{array}\right] \\
& \underset{\sim}{U^{N}}=\left\{\begin{array}{c}
U_{1}^{1} \\
U_{2}^{1} \\
U_{3}^{1} \\
U_{1}^{2} \\
U_{2}^{2} \\
U_{3}^{2} \\
U_{1}^{3} \\
U_{2}^{3} \\
U_{3}^{3}
\end{array}\right\} \quad: \quad \mathbb{P}^{N}=\left\{\begin{array}{c}
P_{1}^{1} \\
P_{2}^{1} \\
P_{3}^{1} \\
P_{1}^{2} \\
P_{2}^{2} \\
P_{3}^{2} \\
P_{1}^{3} \\
P_{2}^{3} \\
P_{3}^{3}
\end{array}\right\}
\end{aligned}
$$

\subsubsection{ELEMENTO TRIANGULAR LINEAR DESCONTÍNUO}

No elemento triangular linear descontínuo, os pontos nodais não coincidem com os nós geométricos localizados nos vértices do elemento. Os pontos nodais 1,2 e 3 estão localizados internamente ao elemento e posicionados sobre segmentos de 
retas paralelas aos lados do elemento. As variáveis são aproximadas por funções de interpolação $\phi^{1}, \phi^{2} \mathrm{e} \phi^{3}$, que possuem valores unitários nos pontos nodais correspondentes 1,2 e 3 , e zero nos demais (Fig. 3.11).

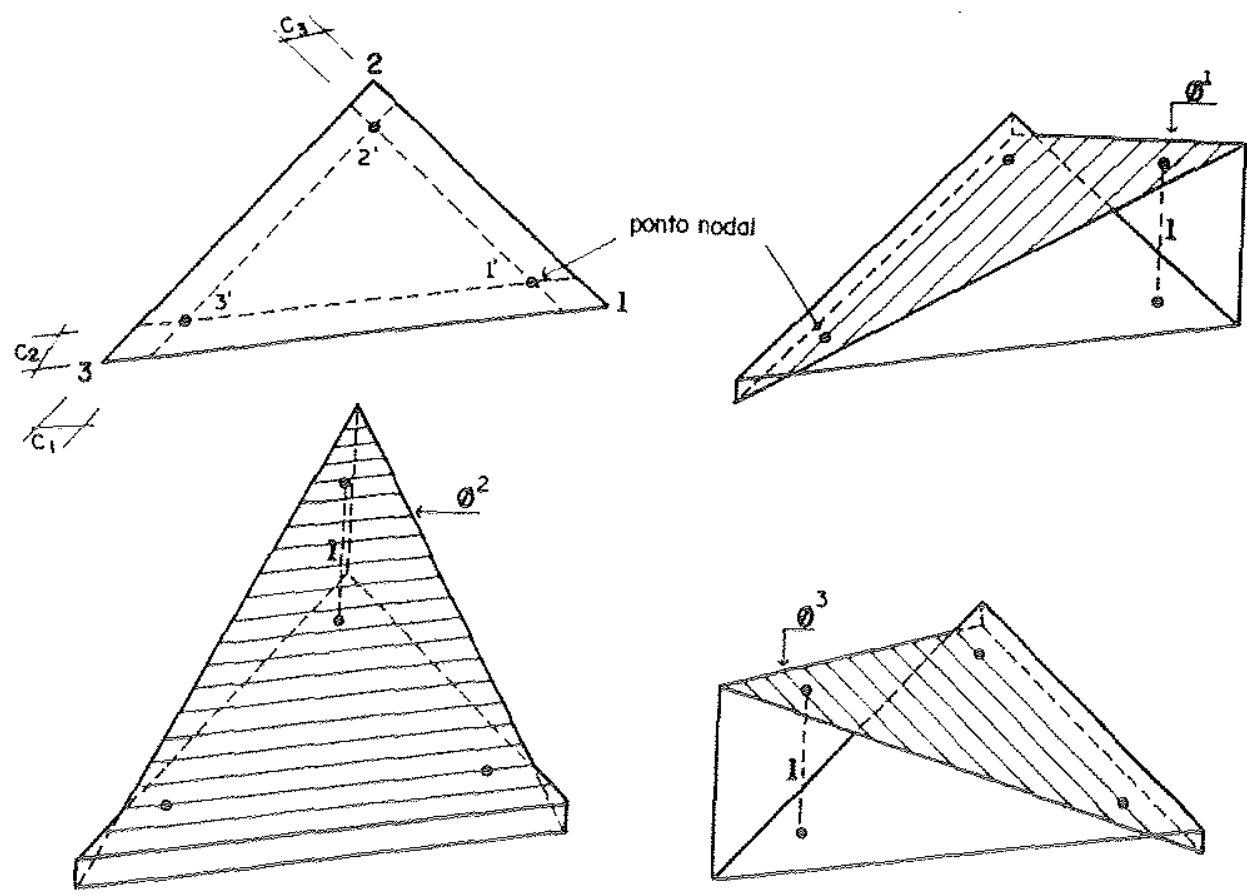

FIGURA 3.11 - Posição e funções de interpolação dos pontos nodais do clemento triangular linear descontinuo.

Os pontos nodais 1,2 e 3 , deslocados para o interior do clemento, fícam definidos $\mathrm{cm}$ função dos parâmetros $\mathfrak{c}_{1}, \mathrm{c}_{2} \mathrm{cc}_{3}$, determinados em relação às coordenadas homogêneas $\xi^{1}, \xi^{2} \mathrm{e} \xi^{3}$, respectivamente, constituindo, assim, um novo sistema de coordenadas homogêneas $\bar{\xi}_{1}, \bar{\xi}_{2}$ c $\bar{\xi}_{3}$ (Fig. 3.12), dadas por:

$$
\begin{aligned}
& \bar{\xi}_{1}=\frac{\xi_{1}-c_{1}}{c_{4}} \\
& \xi_{2}=\frac{\xi_{2}-c_{2}}{c_{4}} \\
& \bar{\xi}_{3}=\frac{\xi_{3}-c_{3}}{c_{4}}
\end{aligned}
$$

sendo que: 


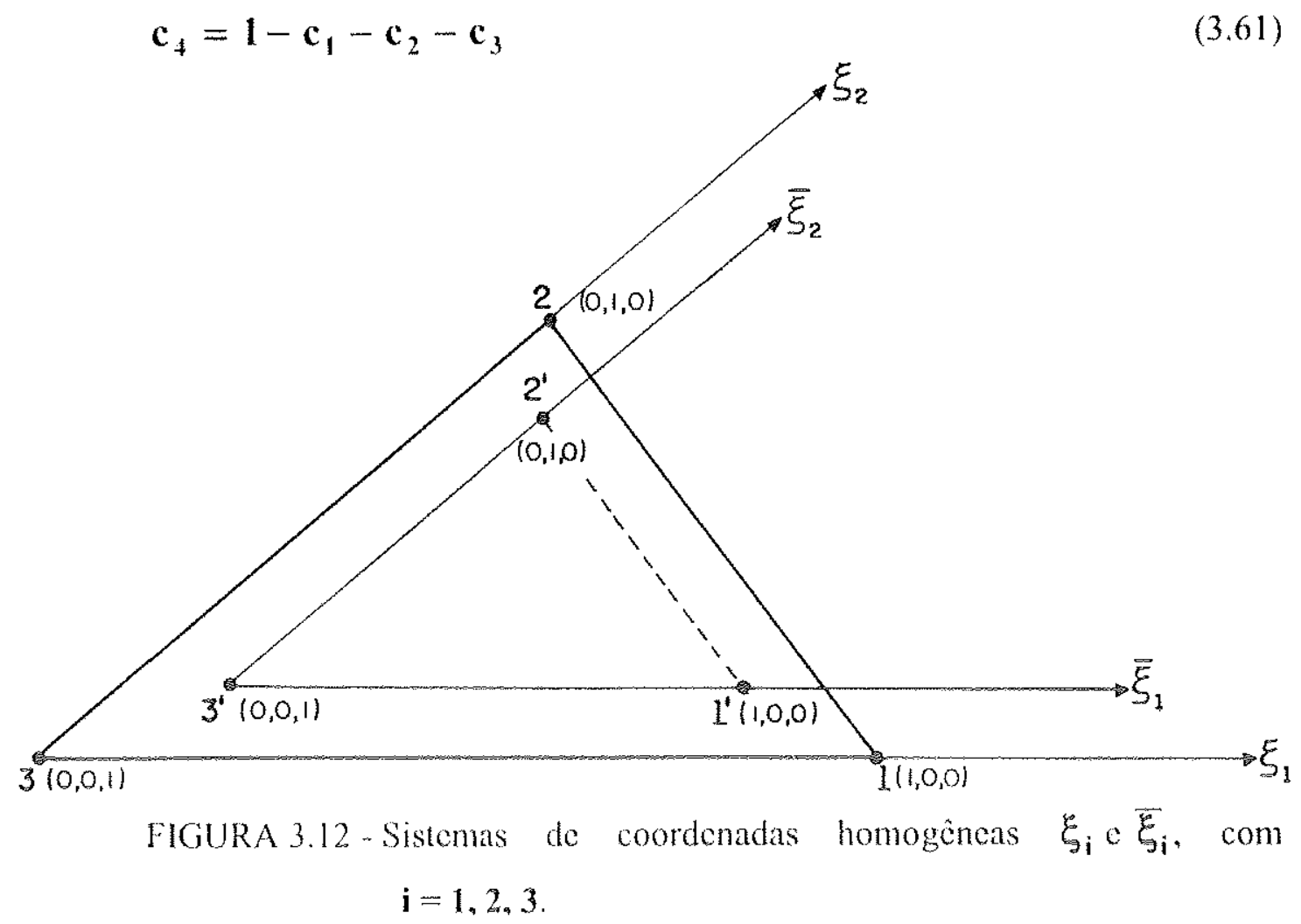

Bascados no trabalho de GIL RODRIGUEZ (1986), BARBIRATO (1991) e TEJERINA CALDERÓN (1996) adotaram:

$$
c_{1}=c_{2}=c_{3}=0,1625
$$

As funções interpoladoras empregadas para aproximar as variáveis são dadas por:

$$
\begin{aligned}
& \phi^{\prime}=\bar{\xi}_{1} \\
& \phi^{2}=\bar{\xi}_{2} \\
& \phi^{3}=\bar{\xi}_{3}
\end{aligned}
$$

Para o clemento triangular linear descontínuo, os deslocamento e forças de superficic são dados por:

$$
\begin{aligned}
& \boldsymbol{l}=\Phi^{\mathrm{T}}(\bar{\xi}) U^{\mathrm{N}} \\
& \mathrm{p}=\bar{\Phi}^{\mathrm{T}}(\bar{\xi}) \mathbf{D}^{\mathrm{N}}
\end{aligned}
$$

onde: 
$\underset{\sim}{\mathbf{u}}=\left\{\begin{array}{l}\mathbf{u}_{1} \\ \mathbf{u}_{2} \\ \mathbf{u}_{3}\end{array}\right\} \quad ; \quad \underset{\sim}{\mathbf{p}}=\left\{\begin{array}{l}\mathbf{p}_{1} \\ \mathbf{p}_{2} \\ \mathbf{p}_{3}\end{array}\right\}$

$\underset{\sim}{\Phi^{\mathrm{T}}}=\left[\begin{array}{ccccccccc}\phi^{1} & 0 & 0 & \phi^{2} & 0 & 0 & \phi^{3} & 0 & 0 \\ 0 & \phi^{1} & 0 & 0 & \phi^{2} & 0 & 0 & \phi^{3} & 0 \\ 0 & 0 & \phi^{1} & 0 & 0 & \phi^{2} & 0 & 0 & \phi^{3}\end{array}\right]$

$U^{N}=\left\{\begin{array}{c}U_{1}^{1} \\ U_{2}^{1} \\ U_{3}^{1} \\ U_{1}^{2} \\ U_{2}^{2} \\ U_{3}^{2} \\ U_{1}^{3} \\ U_{2}^{3} \\ U_{3}^{3}\end{array}\right\}: \quad \mathbb{P}^{N}=\left\{\begin{array}{c}P_{1}^{1} \\ P_{2}^{1} \\ P_{3}^{1} \\ P_{1}^{2} \\ P_{2}^{2} \\ P_{3}^{2} \\ P_{1}^{3} \\ P_{2}^{3} \\ P_{3}^{3}\end{array}\right\}$

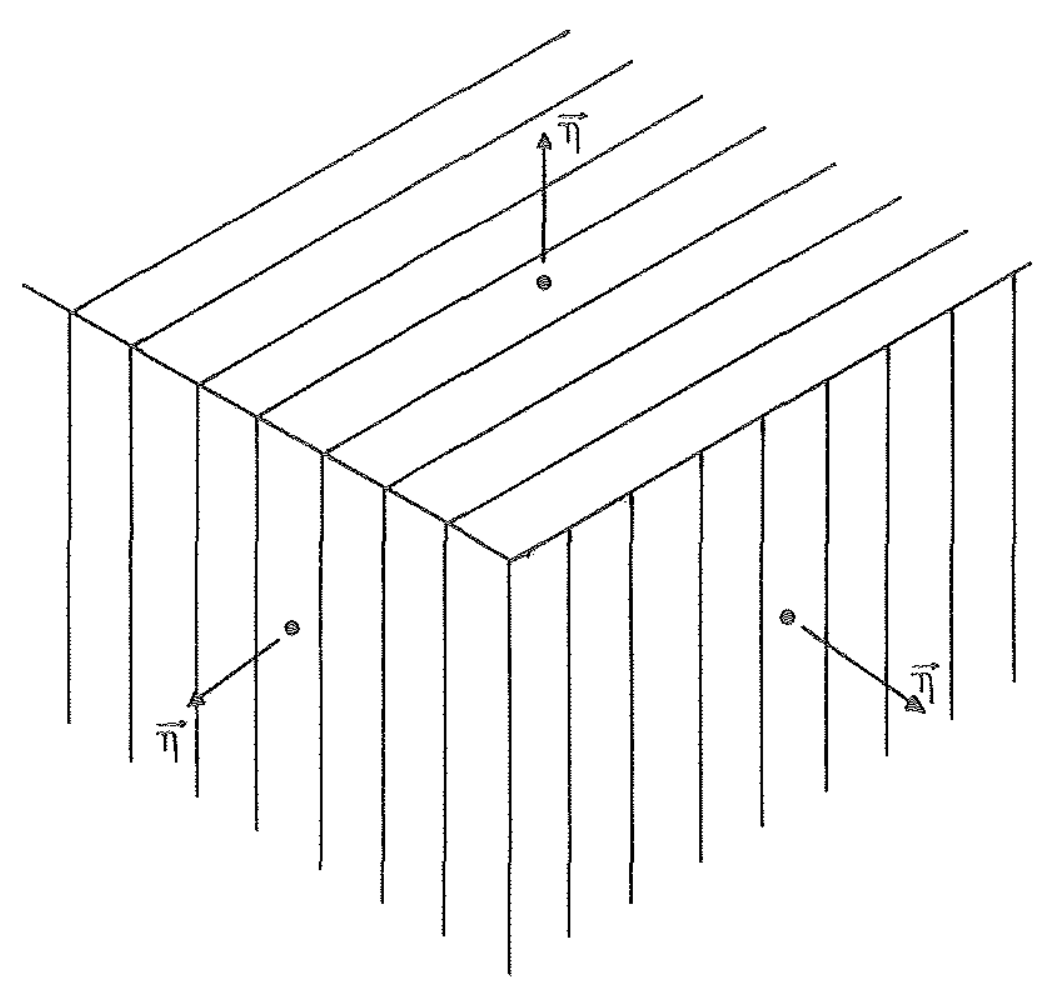

FIGURA 3.13-Descontinuidades no contomo de um sólido tridimensional. 
O emprego deste lipo de elemento triangular linear, dito descontínuo, permite que as variáveis scjam descontinuas em pontos do contorno correspondentes a nós adjacentes. Isto ocorre em situações onde existam vinculações diferentes entre clementos que concorram para um mesmo nó ou angulosidades, como, por excmplo, em arestas e cantos como mostrado a Fig. 3.13. Neste casos, o clemento triangular linear descontínuo é mais adequado, aprescntando resultados com boa aproximação. No entanto, a utilização exclusiva deste clemento para discretizar todo o contorno acarreta um aumento signilicativo no número de nós c, por conseqüência, no número de equações do sistema.

\subsection{INTEGRAÇÃO SOBRE OS ELEMENTOS DE CONTORNO}

Neste trabalho, as forças volumétricas b serão negligenciadas, mesmo sabendo que, em geral, apresentem valores conhecidos. Assim, serão consideradas apenas as variáveis de contorno do sólido tridimensional.

Assim. a equação (3.8), passa a representa apenas o contomo do sólido discretizado em "J" elementos, c toma a forma:

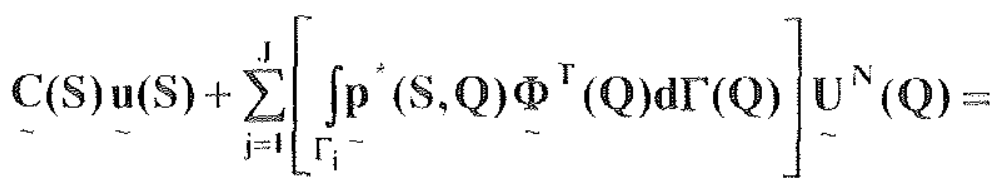

$$
\begin{aligned}
& \sum_{j=1}^{j}\left[\int_{r_{j}} u^{*}(S, Q) \Phi^{T}(Q) d \Gamma(Q)\right] \mathbb{P}^{N}(Q)
\end{aligned}
$$

Os dois termos integrais, explicitados na eq. (3.69), podem ser definidos, respectivamentc como:

$$
\mathbf{h}=\int_{\Gamma_{\mathrm{j}}} \mathrm{p}^{*}(\mathrm{~S}, Q) \underset{\sim}{\Phi^{\mathrm{T}}}(\mathrm{Q}) \mathrm{d} \Gamma(\mathbb{Q})
$$




$$
\underset{\sim}{\mathbf{g}}=\int_{\Gamma_{j}}^{\mathbf{u}^{*}}{ }^{*}(S, Q) \underset{\sim}{\Phi^{\mathrm{T}}}(\mathbf{Q}) \mathbf{d} \Gamma(\mathbf{Q})
$$

As posiçoes do ponto de aplicação $\mathbf{S}$ (ponto lonte) e do ponto resposta $\mathbf{Q}$ (ponto campo), em relação ao elemento a ser integrado, definem o tipo de integração mais apropriado, podendo ocorrer duas situaçoes: quando os pontos $\mathbf{S}$ c $\mathbf{Q}$ pertencem a elementos distintos e quando pertencen ao mesmo elemento, denominadas, respectivamente, de integração não singular e integração singular.

\subsubsection{INTEGRACÃO NÃO SINGULAR}

No caso em que os pontos $\mathrm{Se} Q$ pertençam a elementos distintos, pode-se utilizar a integração numérica para elementos triangulares apresentada por HAMMER el al. (1956), que ć cletuada $\mathrm{cm}$ relaçào às coordenadas triangulares homogêneas $\xi_{i}$; deste modo, a equação (3.69) pode ser escrita como:

$$
\mathbf{h}=|\mathbf{G}| \int_{0}^{1}\left(\int_{0}^{1-\xi_{2}^{2}}\left(\xi_{1}, \xi_{2}, \xi_{3}\right) \mathrm{d} \xi_{1}\right) \mathrm{d} \xi_{2} \text {. }
$$

que, representada na forma de somatório, lica:

$$
\mathbf{h}=|\mathbf{G}| \sum_{i=1}^{n} \mathbf{f}\left(\xi_{1} i, \xi_{2}, \xi_{3}^{i}\right) w_{i}
$$

Nesta equação, tem-se:

G| : jacobiano da transformação;

n : número de pontos de integração;

$\xi_{1}, \xi_{2}, \xi_{3} \quad$ : coordenadas homogêneas dos pontos de integração;

w $_{i} \quad$ pesos associados aos pontos de integração;

$\int_{\sim 1}\left(\xi_{1}, \xi_{2}, \xi_{3}\right)$ : integrando da integral na expressão $(3.70)$.

Utilizando procedimento análogo com a cquação (3.71), obtém-se: 


$$
\underset{\sim}{g}=|\mathbf{G}| \sum_{i=1}^{\mathrm{n}} \mathbf{f}\left(\xi_{1}^{\mathrm{i}}, \xi_{2}^{i}, \xi_{3}^{i}\right) \mathbf{w}_{i}
$$

sendo $f\left(\xi_{1}, \xi_{2}, \xi_{3}\right)$ o integrando da integral na expressão (3.71).

Este processo de integraçào encontra-se disponível na literatura para o emprego de até treze pontos de integração, com os quais se obtêm valores exatos para funções polinomiais de sétimo grau. Contudo, mesmo com esse limitado número de pontos de integração, os resultados são satisfatórios, especialmente quando os pontos $\mathbf{S}$ e $\mathbf{Q}$ encontram-se suficientemente afastados. No entanto, quando esses pontos estão próximos, mesmo pertencendo a clementos diferentes, há a necessidade de um número maior de pontos de integração para se obter resultado mais preciso.

Uma alternativa para aumentar o número de pontos de integração, segundo NAKAGUMA (1979), consiste en subdividir o clemento triangular $\mathrm{cm}$ subelementos, em cada un dos quais podem ser utilizados até treze pontos de integração. Deste modo, o número de pontos de integração no elemento triangular ć incrementado de acordo com o número de subdivisões em subclementos.

Obviamente, com esses subelementos nào serão criados quaisquer nós adicionais no elemento; eles apenas serão empregados para o cálculo das integrais numéricas nos subelementos, sem modificar o número total de incógnitas do sistema de equaçõcs.

Para a divisão do elemento em subelementos, são necessários dois sistemas de coordenadas homogeneas diferentes; $\xi_{i}$, associada ao elemento, $c \bar{\xi}_{i}$, associada ao subclemento, como mostrado à Fig. 3.14. 


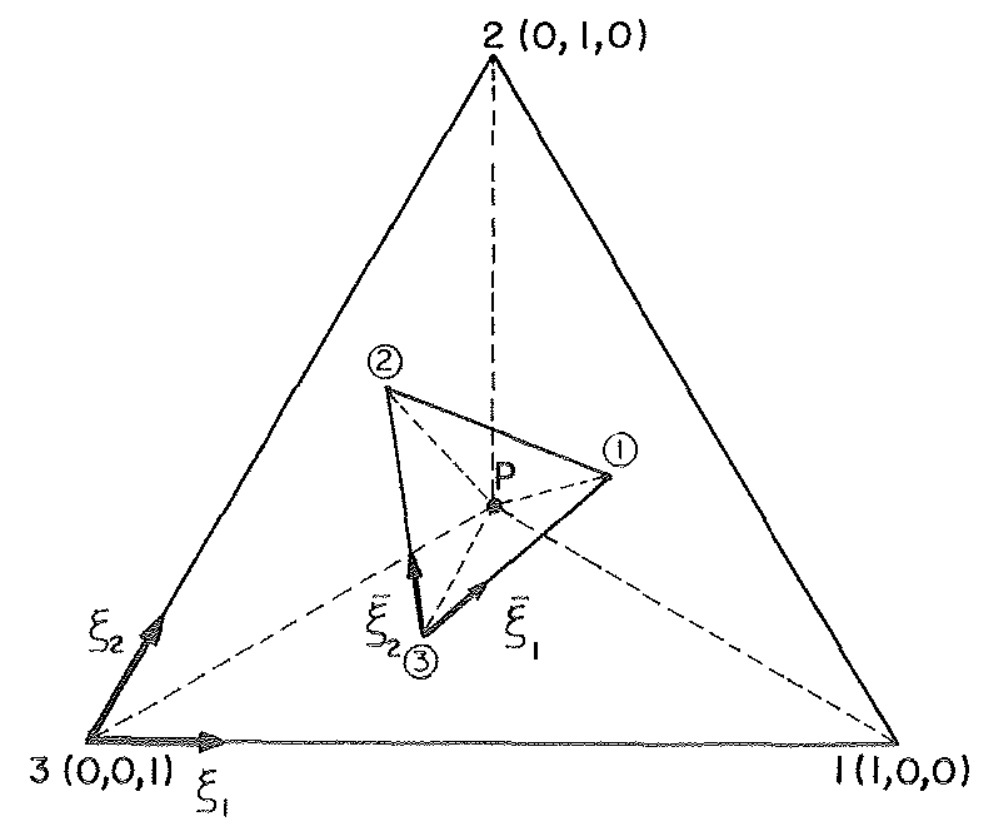

FIGURA 3.14 - Coordenadas triangulares homogêncas do elemento e subclemento.

As coordenadas cartesianas $\mathbf{X}$ de um ponto $\mathbf{P}$ pertencente a um elemento (Fig.

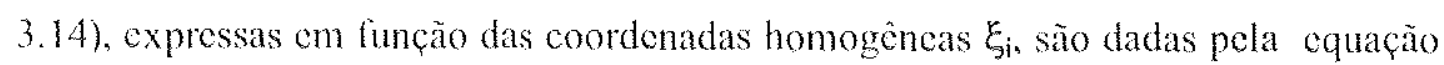
(3.31) já apresentada, isto c:

$$
\underset{\sim}{X} \underset{\sim}{\Psi^{\top}}(\xi) \underset{\sim}{\mathbb{X}^{N}}
$$

Analogamente, as mesmas coordenadas cartesianas $\mathbf{X}$ do ponto $\mathbf{P}$ pertencente ao clemento (Fig. 3.14), expressas agora em função das coordenadas homogêneas $\bar{\xi}_{\mathrm{i}}$, podem ser representadas como:

$$
\mathrm{X}=\bar{\Psi}^{\mathrm{T}}(\bar{\xi}) \mathrm{x}^{\bar{N}}
$$

sendo:

$\vec{\sim}^{\mathrm{T}}(\bar{\xi})$ : as lunções de interpolação do subclemento; 
$\mathbf{x}^{\bar{N}} \quad$ : as coordenadas cartesianas dos nós do subelemento.

A partir da equação (3.31), e escrevendo as coordenadas cartesianas dos nós do subetemento $\mathrm{x}^{\mathrm{N}} \mathrm{em}$ função das coordenadas cartesianas dos nós do elemento $\mathbf{X}^{\mathrm{N}} \cdot \operatorname{tcm}-\mathrm{sc}:$

$$
\underset{\sim}{\mathbf{x}^{\bar{N}}}=\underline{\sim}^{\top}\left(\xi^{\bar{N}}\right) \underset{\sim}{\underset{X}{N}}
$$

onde $\xi^{\bar{N}}$ são as coordenadas $\xi$ dos nós do subelemento.

Substituindo-se a equação (3.76) na equação (3.75), obtém-se:

$$
\underset{\sim}{x}=\bar{\Psi}^{\mathrm{T}}(\bar{\xi}) \underset{\sim}{\Psi^{\mathrm{T}}}\left(\xi^{\bar{N}}\right) X^{N}
$$

Igualando-se as coordenadas cartesianas $\mathbf{x}$ dadas pelas equações $(3.31) \mathrm{c}$ (3.77), tem-se:

$$
\underset{\sim}{\Psi}(\xi)=\underset{\sim}{\Psi}(\xi \vec{\xi}) \bar{\Psi}(\vec{\xi})
$$

Para os clementos com função interpoladora com variação linear, a equação (3.78), escrita $\mathrm{cm}$ forma explicita, toma-se:

$$
\left\{\begin{array}{l}
\xi_{1} \\
\xi_{2} \\
\xi^{\prime}
\end{array}\right\}=\left[\begin{array}{lll}
\xi_{1}^{(1)} & \xi_{1}^{(2)} & \xi_{1}^{(3)} \\
\xi_{2}^{(1)} & \xi_{2}^{(2)} & \xi_{2}^{(3)} \\
\xi_{3}^{(1)} & \xi_{3}^{(2)} & \xi_{3}^{(3)}
\end{array}\right]\left\{\begin{array}{c}
\bar{\xi}_{1} \\
\overline{\xi_{2}} \\
\overline{\xi_{3}}
\end{array}\right\}
$$

$\xi_{i}$ e $\overline{\xi_{i}}$ são as coordenadas homogêneas do elemento e subelemento, respectivamente, $\mathrm{c} \xi_{\mathbf{i}}^{(\mathbf{j})}$, as coordenadas $\xi_{\mathbf{i}}$ de cada um dos nós $\mathbf{j}$ do subelemento.

As coordenadas $\overline{\xi_{i}}$ serão dadas pelas coordenadas homogêncas dos pontos de integração para cada subelemento e a matriz $\underset{\sim}{\Psi}\left(\xi^{\bar{N}}\right)$ será comada pelas coordenadas $\xi_{i}$ dos nós de cada subetemento.

A subdivisão do elemento em subelementos pode ser feita de forma que estes scjam obtidos com as mesmas características geométricas do elemento gerador, como mostra a Fig. 3.15. Isto se consegue dividindo os lados do clemento em partes iguais 
e unindo os pontos assim determinados por segmentos de retas paralelos aos lados do elemento. Deste modo, dividindo-se os lados do elemento em duas partes iguais, serão obtidos quatro subelementos; em três partes iguais, nove subelementos; $\mathrm{c}$, assim, sucessivamente, podendo-se observar uma variação quadrática no número de subelementos gerados, em relação ao número de subdivisões dos lados do elemento.

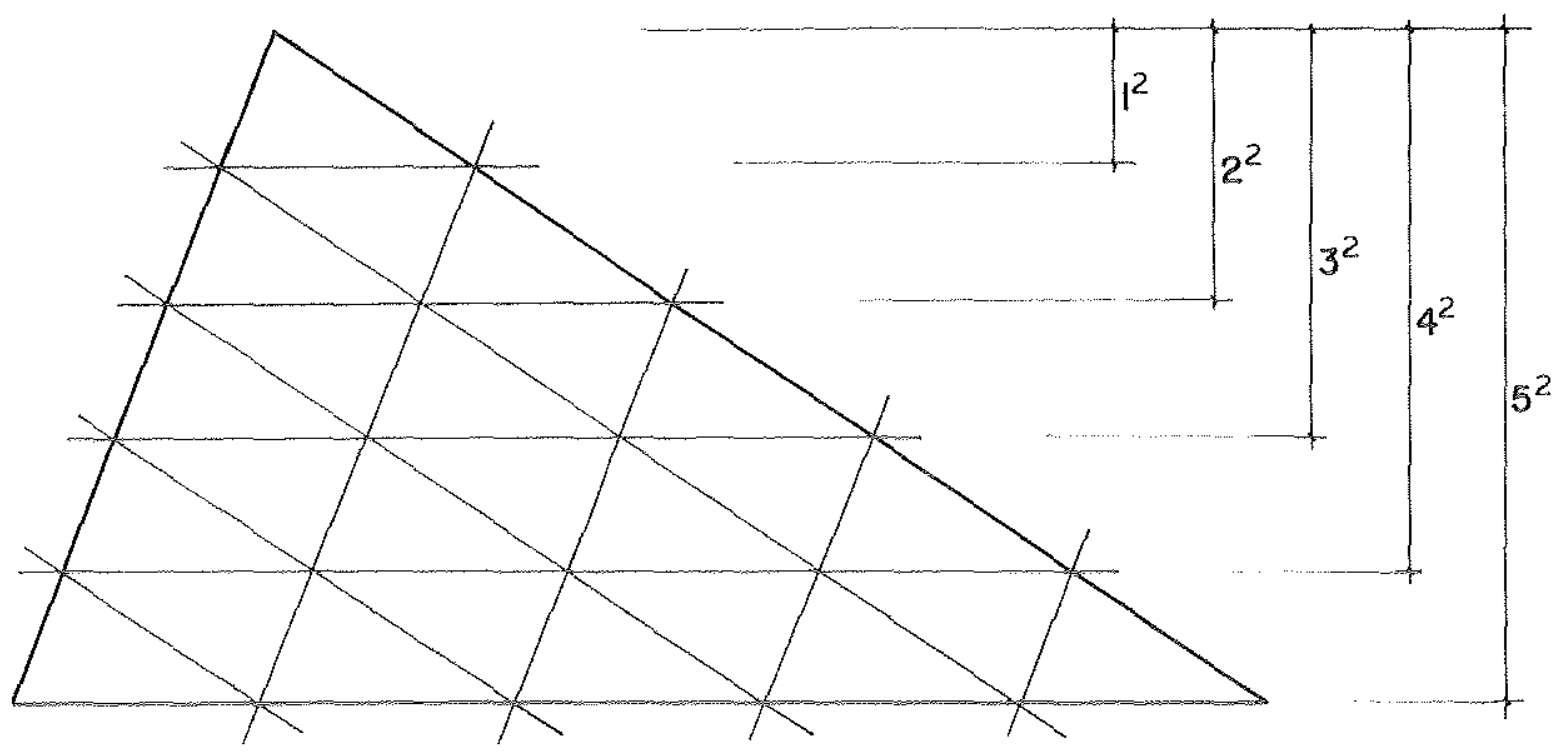

FIGURA 3.15-Divisão de um elemento em subelementos.

Para exemplificar, vejamos um elemento dividido en quatro subelementos, apresentados na Fig. 3.16, sendo que as matrizes $\Psi\left(\xi^{N}\right)$ apresentam os seguintes vatores:

$\left[\begin{array}{ccc}1 & 1 / 2 & 1 / 2 \\ 0 & 1 / 2 & 0 \\ 0 & 0 & 1 / 2\end{array}\right]$ subelemento 1

$\left[\begin{array}{ccc}1 / 2 & 0 & 0 \\ 1 / 2 & 1 & 1 / 2 \\ 0 & 0 & 1 / 2\end{array}\right]$

subclemento 2 
$\left[\begin{array}{ccc}1 / 2 & 0 & 1 / 2 \\ 1 / 2 & 1 / 2 & 0 \\ 0 & 1 / 2 & 1 / 2\end{array}\right] \quad\left[\begin{array}{ccc}1 / 2 & 0 & 0 \\ 0 & 1 / 2 & 0 \\ 1 / 2 & 1 / 2 & 1\end{array}\right]$

subelemento 3

subelemento 4

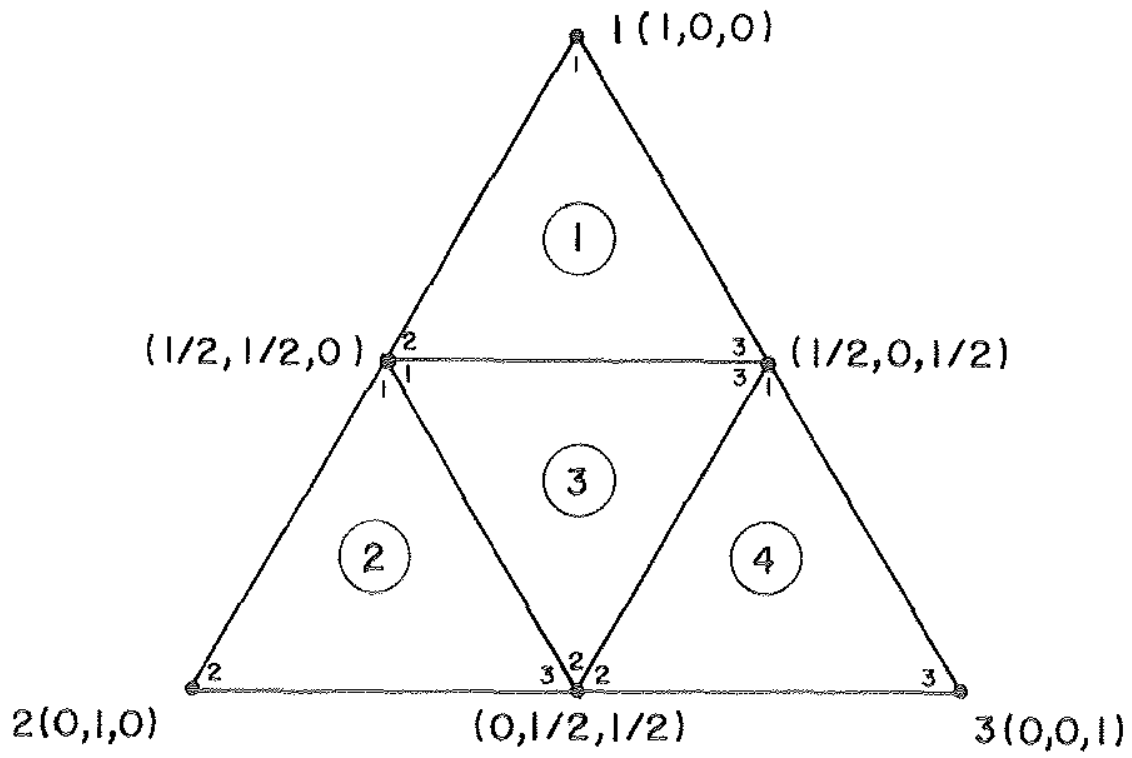

FIGURA 3.16 - Elemento dividido em quatro subelementos.

O número de subclementos pode ser detcminado cm função da distância entre o ponto lonte c o clemento a ser integrado, ou seu centróide. Quanto menor essa distância, maior scrá o númcro de subclementos utilizados. NAKAGUMA (1979) cmpregou sete pontos de integração em cada subelemento c admitiu os seguintes intervalos para determinar o número de subelementos:

$$
\begin{array}{ll}
\mathbf{0} \leq \overline{\mathrm{R}} \leq \overline{\mathrm{L}} & 9 \text { subelementos; } \\
\overline{\mathrm{L}}<\overline{\mathrm{R}} \leq 2 \overline{\mathrm{L}} & \text { 6 subclementos; } \\
\overline{\mathrm{R}}>2 \overline{\mathrm{L}} & \text { 3 subelementos. }
\end{array}
$$

Aqui, $\bar{R}$ ć a distância entre o ponto lonte c o centróide do elemento e $\overline{\mathrm{L}}$, a média dos comprimentos dos lados do elemento.

Com o objetivo de obter resultados mais precisos, optou-se, neste trabalho, por utilizar também sete pontos de integraçào en cada subelemento, mas se 
adotando os seguintes intervalos para a determinação do número de subelementos, conforme TEGERINA CALDERÓN (1996):

$$
\begin{array}{ll}
\mathbf{0} \leq \overline{\mathbf{R}} \leq \overline{\mathbf{L}} & 25 \text { subelementos; } \\
\overline{\mathbf{L}}<\overline{\mathbf{R}} \leq \mathbf{2} \mathbf{L} & 16 \text { subelementos; } \\
\mathbf{2} \overline{\mathbf{L}}<\overline{\mathbf{R}} \leq \mathbf{4} \overline{\mathbf{L}} & 9 \text { subelementos; } \\
\overline{\mathbf{R}}>\mathbf{4} \overline{\mathbf{L}} & 4 \text { subelementos. }
\end{array}
$$

Tendo o elemento sido dividido em subclementos, as equaçòes (3.73) e (3.74) assumem a forma:

$$
\begin{aligned}
& \mathbf{h}=\sum_{\mathbf{k}=1}^{N s}|\overline{\mathbf{G}}| \sum_{i=1}^{\mathrm{n}} \mathbf{f}\left(\xi_{1}^{i}, \xi_{2}^{i}, \xi_{3}^{i}\right) \mathbf{w}_{i}, \\
& \mathbf{g}=\sum_{\mathrm{k}=1}^{N \mathrm{~s}}|\mathbf{G}| \sum_{i=1}^{\mathrm{n}} \mathbf{f}\left(\xi_{1}^{i}, \xi_{2}^{i}, \xi_{3}^{i}\right) \mathbf{w}_{i}
\end{aligned}
$$

sendo, $\bar{G}$ o jacobiano da transformaçào realizada.

Outra altemativa para aumentar o número de pontos de integração foi sugerida por TELLES (1986), que consiste em transformar o clemento de domínio triangular em quadrangular, podendo-se, assim, recorrer aos pontos de integração da conhecida quadratura de Gauss, aplicados cm duas direçōes ortogonais.

\subsubsection{INTEGRAÇÃO SINGULAR}

Quando os pontos $\mathbf{S}$ (ponto fonte) e $\mathbf{Q}$ (ponto campo) localizam-se no mesmo clemento, a integração numérica, segundo Hammer, não permite uma boa aproximação, devido à singularidade existente, indicando a necessidade de se recorrer a um procedimento alternativo.

Um artificio utilizado para solucionar esse problema consiste no emprego de um sistema local de coordenadas cilíndricas $(r, \theta, z)$, com origem no ponto $\mathbf{S}$ c o cixo z perpendicular ao plano do elemento, como mostrado à Fig. 3.17. 


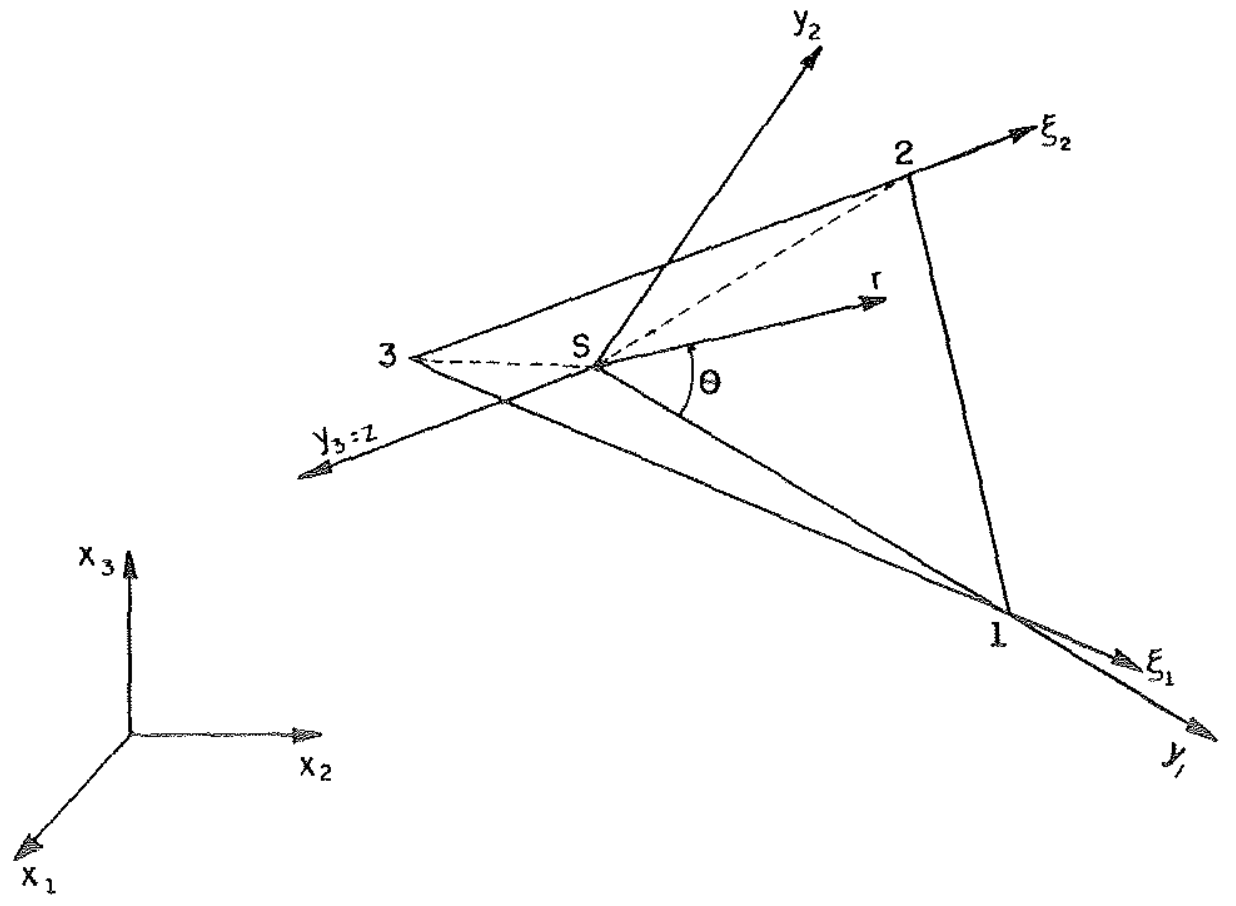

FIGURA 3.17 - Sistema de coordenadas locais cartesiano e cilíndrico.

Procedendo-se desta forma, reduz-se a ordem da singularidade nas integrais das submatrizes $g$ e h, que são $\frac{1}{\mathrm{r}} \mathrm{e} \frac{1}{\mathrm{r}^{2}}$, respectivamente, c o clemento a ser integrado licará em função apenas de r e $\theta$; assim, as equaçōes (3.73) e (3.74) poderão ser rescritas como:

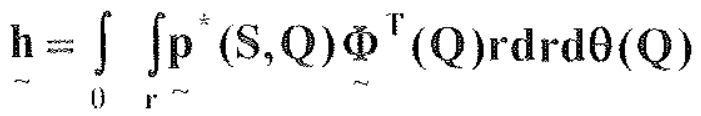

$$
\begin{aligned}
& \underset{\sim}{g}=\int_{0} \int_{\mathrm{r}} \mathbf{u}_{\sim}^{*}(\mathrm{~S}, \mathrm{Q}) \stackrel{\Phi}{\sim}^{\mathrm{T}}(\mathrm{Q}) \mathrm{rdr} \mathrm{d} \theta(\mathrm{Q}),
\end{aligned}
$$

sendo :

$$
d \Gamma=\operatorname{rdrd} \theta
$$

As soluçōes fundamentais de Mindlin, escritas em função de $\mathbf{u}_{\sim}^{*} \mathfrak{e} \mathbf{p}_{\sim}^{*}$, podem ser apresentadas sob a seguinte forma: 


$$
\begin{aligned}
& \mathbf{p}^{*}=\mathbf{k}_{\mathrm{s}} \frac{\mathbf{k}_{3} \mathbf{r}_{\mathrm{i}}+\mathbf{k}_{4} \mathbf{r}_{\mathrm{i}, \mathbf{r}_{\mathrm{j}} \mathbf{r}_{\mathrm{k}}}}{\mathbf{r}^{2}}+\mathbf{k}_{\mathrm{s}} \mathbf{f}_{\mathrm{p}}(\mathbf{R}) \\
& \mathbf{u}^{*}=\mathbf{k}_{\mathrm{d}} \frac{\mathbf{k}_{\mathrm{i}}+\mathbf{k}_{2} \mathbf{r}_{\mathrm{i}, \mathrm{r}} \mathbf{r}_{\mathrm{j}}}{\mathbf{r}}+\mathbf{k}_{\mathrm{d}} \mathbf{f}_{\mathrm{u}}(\mathbf{R}),
\end{aligned}
$$

onde:

$\mathbf{k}_{\mathbf{i}}$ são coeficientes constantes;

$\mathbf{r}_{\mathrm{j},}, \mathbf{r}_{\mathrm{j}} \mathrm{c} \mathbf{r}_{\mathrm{k}}$ são os cossenos diretores do vetor $\overrightarrow{\mathbf{r}}$;

$\mathbf{f}_{\mathrm{t}}(\mathbf{R})$ e $\mathbf{f}_{\mathrm{p}}(\mathbf{R})$, sào funções relacionadas à distância entre o pontos campo $\mathbf{Q}$ e o ponto $S^{\prime}$, sendo este último simétrico ao ponto lonte $S$, em relação ao plano $\mathbf{x}_{3}=\mathbf{0}($ veja Fig. 2.10$)$.

As integrais que contêm as funções $\mathbf{f}_{11}(\mathbf{R})$ c $\mathbf{f}_{p}(\mathbf{R})$ tem comportamento distintos dependendo da posição do ponto $\mathbf{S}$. Assim, quando o ponto $\mathbf{S}$ (fonte) não está contido no plano $\mathrm{x}_{3}=\mathbf{0}$, solução fundamental de Mindlin, as integrais são não singulares, visto que o ponto $\mathbf{S}$ ' fica sempre fora do elemento; portanto as integrais poderão ser calculadas como indicado no item anterior, isto é, resolvidas como integrais numéricas. No entanto, quando o ponto $S$ está contido no plano $x_{3}=\mathbf{0}$ (solução lundamental de Boussinesq-Cerruti), o ponto $\mathbf{S}$ ' coincide com o ponto $\mathbf{S} \mathrm{c}$, portanto $\mathbf{R}=\mathbf{r}$. Nestc caso, as integrais são singulares e deve-se observar os procedimentos que a seguir se indica para a solução dessas integrais.

Considerando-se apenas os termos em $r$ das soluçōes fundamentais $(3.85) \mathrm{c}$ (3.86); as integrais (3.83) e (3.84) assumem a seguinte forma:

$$
\begin{aligned}
& \mathbf{h}=\int_{0} \int_{r} k_{h} \frac{1}{r} \Phi^{T}(Q) d r d \theta(Q) \\
& g=\int_{0} \int_{r} k_{g} \stackrel{\Phi}{\sim}^{T}(Q) d r d \theta(Q)
\end{aligned}
$$

nestas, têm-se:

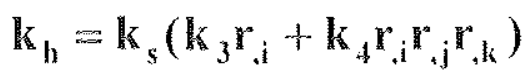

$$
\begin{aligned}
& \mathbf{k}_{g}=\mathbf{k}_{d}\left(k_{1}+k_{2} r^{r_{i}} r_{, j}\right)
\end{aligned}
$$


As funções interpoladoras $\Phi^{\mathrm{T}}$ para um elemento linear, cxpressas em termos de coordenadas cartesianas locais $\mathbf{y}$, tomam a seguinte forma geral, ja apresentada anteriormente:

$$
\xi_{i}=a_{i} y_{1}+b_{i} y_{2}+c_{i} \quad(i=1,2,3)
$$

sendo que:

$$
\begin{array}{ll}
a_{i}=\frac{1}{2 A}\left(y_{2}^{j}-y_{2}^{k}\right) & (i=1,2,3) \\
b_{i}=\frac{1}{2 A}\left(y_{1}^{k}-y_{1}^{j}\right) & (j=2,3,1) \\
c_{i}=\frac{1}{2 A}\left(y_{1}^{j} y_{2}^{k}-y_{1}^{k} y_{2}^{j}\right) & (k=3,1,2)
\end{array}
$$

onde, $\mathbf{i}, \mathbf{j}$ c $\mathbf{k}$ são tomados em seqüência c ciclicamente, como indicado acima.

Transformando-se as coordenadas cartesianas y em coordenadas cilíndricas, têm-sc:

$$
\begin{aligned}
& y_{1}=r \cos \theta \\
& y_{2}=r \operatorname{sen} \theta \\
& y_{3}=x=0
\end{aligned}
$$

Escrevendo-se a equação $(3.24) \mathrm{em}$ coordenadas cilíndricas e substituindo-a nas cquações (3.88) e (3.89), obtém-se:

$$
\begin{aligned}
& h=\int_{\theta} \int_{r} k_{h} \frac{1}{r}\left(a_{i} r \cos \theta+b_{i} r \operatorname{sen} \theta+c_{i}\right) d r d \theta(Q) \\
& g=\int_{\theta} \int_{r} k_{g}\left(a_{i} r^{r} \cos \theta+b_{i} r^{r e n} \theta+c_{i}\right) d r d \theta(Q)
\end{aligned}
$$

onde, $\mathrm{i}=1,2,3$. 


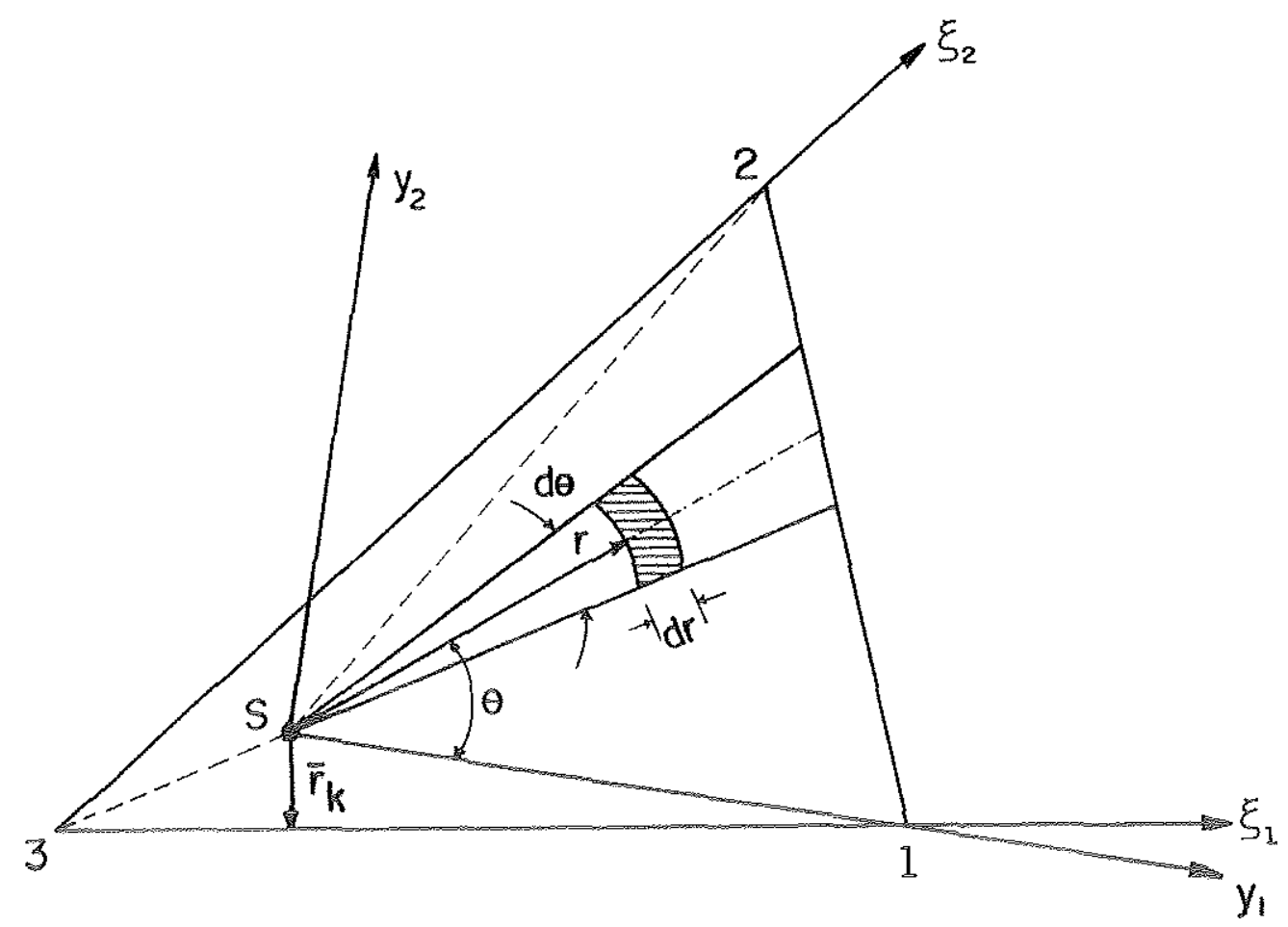

FIGURA 3.18 - Esquema de integraçăo $\mathrm{cm} r$.

Constata-se que a utilizaçào de coordenadas cilíndricas eliminou a singularidade nas integrais da submatriz $\mathbf{g}$, permitindo-se integrar analiticamente em $\mathbf{r}$ (Fig. 3.18), ficando:

$$
g=\int_{0} k_{g}\left(a_{i} \frac{\bar{r}_{k}^{2}}{2} \cos \theta+b_{i} \frac{\bar{r}_{k}^{2}}{2} \operatorname{sen} \theta+c_{i} \bar{r}_{k}\right) d \theta(Q)
$$

sendo que $\overline{\mathbf{r}}_{\mathrm{k}}$ é a cquação do lado $\mathrm{k}$ do elemento triangular, dada por:

$$
\overline{\mathrm{r}}_{\mathrm{k}}^{\mathrm{x}}=-\frac{\mathrm{c}_{\mathrm{k}}}{\mathrm{a}_{\mathrm{k}} \cos \theta+b_{k} \operatorname{sen} \theta}
$$

A singularidade nas integrais da submatriz $\mathbf{h}$ foi reduzida de $\frac{1}{r^{2}}$ para $\frac{1}{r}$; portanto, será necessário que a integração seja cfetuada no sentido do valor principal, isto ć: 


$$
\begin{aligned}
& h=\lim _{\varepsilon \rightarrow 0}\left[\int_{0} \int_{\varepsilon}^{\bar{r}} k_{h}\left(a_{i} \cos \theta+b_{i} \operatorname{sen} \theta+\frac{c_{i}}{\vec{r}}\right) d r d \theta(Q)\right] \\
& \underset{\sim}{\mathbf{h}}=\int_{\theta} \mathbf{k}_{\mathrm{l}}\left(\mathbf{a}_{\mathrm{i}} \overline{\mathbf{r}}_{\mathrm{k}} \cos \theta+\mathbf{b}_{\mathrm{i}} \overline{\mathbf{r}}_{\mathrm{k}} \operatorname{sen} \theta+\mathbf{c}_{\mathrm{i}} \ln \overline{\mathbf{r}}_{\mathrm{k}}\right) \mathrm{d} \theta(\mathrm{Q})- \\
& \lim _{\varepsilon \rightarrow 0}\left[\int_{0} k_{h} c_{i} \ln \varepsilon d \theta(Q)\right]
\end{aligned}
$$

Integrando-se em relação a $\theta$ o termo com o limite, obtém-se:

$$
\lim _{\varepsilon \rightarrow 0}\left[\int_{0} k_{h} c_{i} \ln \varepsilon d \theta(Q)\right]=\lim _{\varepsilon \rightarrow 0}\left[c_{i} \ln \varepsilon \int_{0}^{2 \pi} k_{h} d \theta(Q)\right]
$$

O cálculo da integral em $\theta$ conduz a valores nulos. uma vez que o integrando $\mathbf{k}_{\mathrm{h}}$ contém uma combinação de lunções de seno $\mathrm{c}$ cosseno de $\theta$. Portanto, recscrevendo a equação $(3.99)$, tem-se:

$$
\mathbf{h}=\int_{0} k_{h}\left(a_{i} \bar{r}_{k} \cos \theta+b_{i} \bar{r}_{k} \operatorname{sen} \theta+c_{i} \ln \widetilde{r}_{k}\right) d \theta(Q)
$$

Após a integração analítica cm $\mathbf{r}$, as equações (3.96) c (3.101), são, geralmente, integradas numericamente $\mathrm{em} \theta$, utilizando-se o processo de Gauss.

Outra forma de calcular as integrais (3.96) e (3.101) é transformando-as cm integrais sobre o contomo do elemento triangular, considerando-se este último como sendo formado por três elementos lineares (Fig. 3.19), através da substituição da diferencial $\mathrm{em} \theta$ pela seguinte relaçào:

$$
\mathbf{d} \hat{\Gamma} \frac{\partial \mathbf{r}}{\partial \hat{\mathbf{n}}}=\mathbf{r d} \theta
$$

sendo que di e $\frac{\partial \hat{r}}{\partial \hat{n}}$ são relativos aos elementos lineares que formam o elemento triangular. 


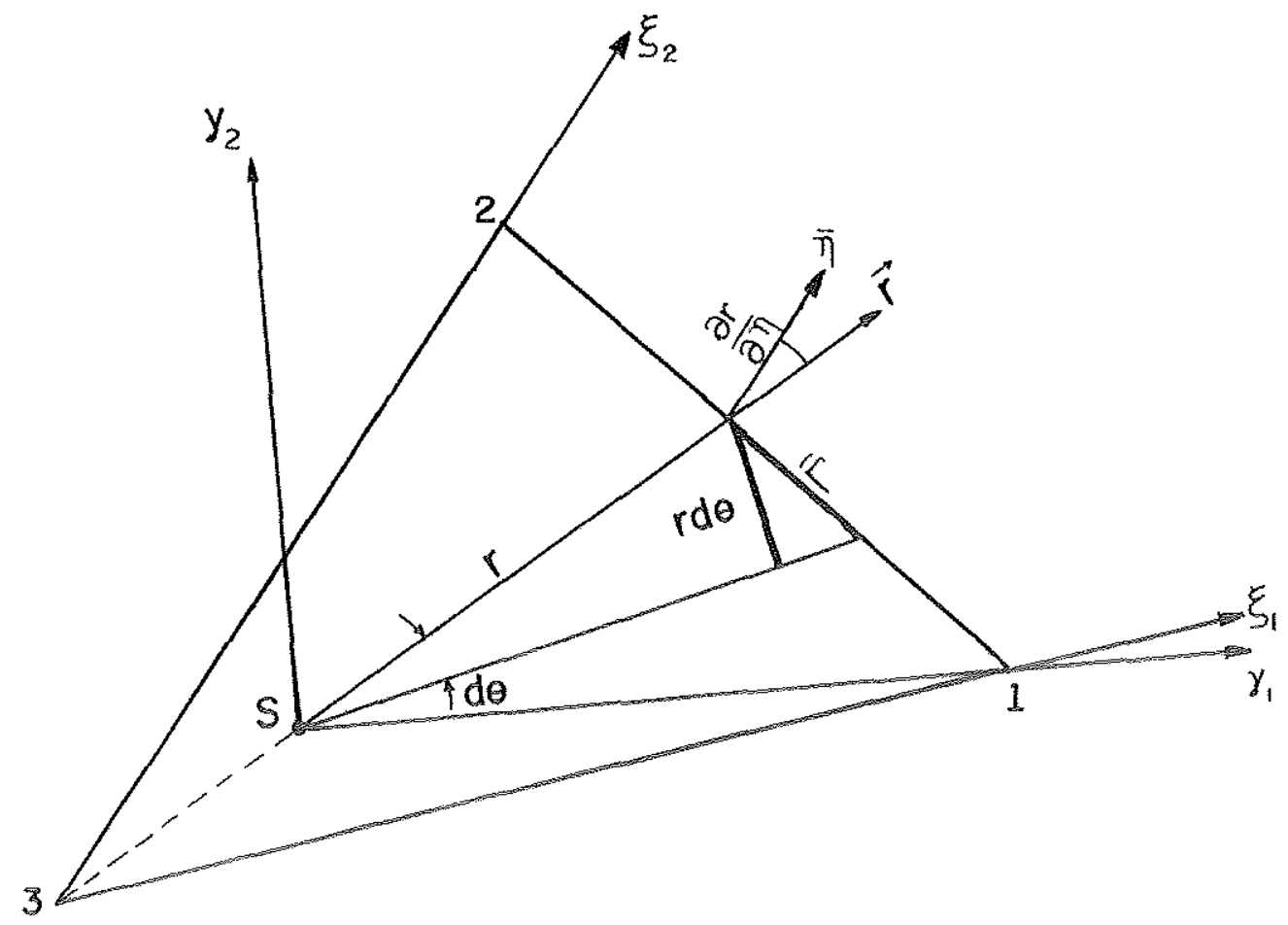

FIGURA 3.19 - Integração para ponto de colocação interno sobre os lados do elemento triangular.

Os lados do elemento triangular formam o "contorno fictício" do mesmo e podem ser considerados como se lossem "elementos de contorno lincares". Assin, as integrais (3.96) e (3.101), assumem a seguinte forma:

$$
\begin{aligned}
& \mathbf{h}=\int_{\tilde{\Gamma}} \mathrm{k}_{\mathrm{h}} \frac{1}{\overline{\mathbf{r}}_{\mathrm{k}}}\left(\mathrm{a}_{\mathrm{i}} \widetilde{\mathbf{y}}_{1 \mathrm{k}}+\mathrm{b}_{\mathrm{i}} \overline{\mathbf{y}}_{2 \mathrm{k}}+\mathrm{c}_{\mathrm{i}} \ln \overline{\mathrm{r}}_{\mathrm{k}}\right) \frac{\partial \mathrm{r}}{\partial \hat{\mathrm{n}}} \mathbf{d} \hat{\Gamma}(\mathbf{Q}) \\
& g=\int_{\tilde{\Gamma}} k_{g}\left(a_{i} \frac{\widetilde{\mathbf{y}}_{1 k}}{2}+b_{i} \frac{\overline{\mathbf{y}}_{2 k}}{2}+\mathbf{c}_{i}\right) \frac{\partial \mathbf{r}}{\partial \hat{n}} d \hat{\Gamma}(\mathbb{Q})
\end{aligned}
$$

Integrando-se (3.103) e (3.104) ao longo dos três lados do elemento triangular, utilizando-se a quadratura de Gauss, obtêm-sc:

$$
h=\sum_{k=1}^{3}|\hat{j}| \sum_{i=1}^{n} \hat{i}\left(\hat{\xi}_{i}\right) w i
$$




$$
\mathbf{g}=\sum_{\mathrm{k}=1}^{3}|\hat{\mathbf{J}}| \sum_{\mathrm{i}=1}^{\mathrm{n}} \hat{\mathbf{f}}\left(\hat{\xi}_{\mathrm{g}}\right) \mathbf{w}_{\mathrm{i}}
$$

sendo que:

k representa cada um dos três clementos lineares;

n é o número de pontos de integração;

$\hat{\xi}_{i}$ são as coordenadas homogeneas dos pontos de integração;

$w_{i}$ são os pesos dos pontos de integração;

$|\hat{\mathbf{J}}|$ co jacobiano da transformação:

$\hat{\mathbf{f}}\left(\hat{\xi}_{\sim \mathrm{i}}\right) \stackrel{\hat{f}}{\hat{\mathbf{h}}}\left(\hat{\xi}_{\mathrm{h}}\right)$ sào os integrandos das integrais (3.103) e (3.104), respectivamente, em função da coordenada homogênca $\hat{\xi}_{\mathrm{i}}$.

Os procedimentos utilizados neste item, embora tenham considerado o ponto $\mathbf{S}$ (fonte) situado no interior do clemento triangular, são também válidos para as situações em que este ponto esteja localizado nos lados ou mesmo nos vértices do mesmo, desde que, haja continuidade tanto na geometria, quanto nas condições de contorno nos elementos envolvidos.

\subsection{MONTAGEM DO SISTEMA DE EQUAÇÕES}

Negligenciando as forças volumétricas, a equação integral de deslocamentos (3.8), toma a forma:

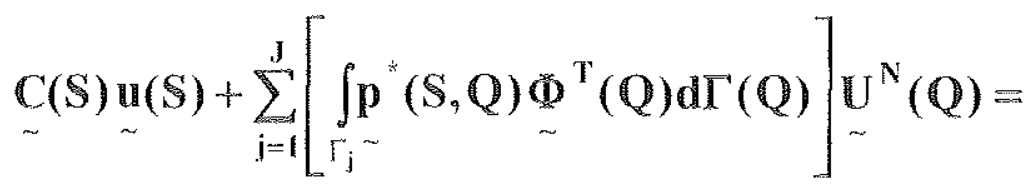

$$
\begin{aligned}
& =\sum_{j=1}^{J}\left[\int_{\Gamma_{i}} \mathrm{u}^{*}(\mathrm{~S}, \mathrm{Q}) \Phi^{\mathrm{T}}(\mathrm{Q}) \mathrm{dT}(\mathrm{Q})\right] \mathrm{P}_{\sim}^{\mathrm{N}}(\mathrm{Q})+
\end{aligned}
$$


Esta equação scrá aplicada $\mathrm{cm}$ um número igual aos pontos nodais do contorno, para formar um sistema de equações compatível com o número de incógnitas, porém, cla não precisa ser aplicada, necessariamente, nos próprios pontos nodais. Assim, no caso de um elemento linear contínuo, em que os pontos nodais estão situados nos vértices, a cquação pode ser aplicada $\mathrm{cm}$ um ponto $\mathbf{S}$ situado no interior do elemento (Fig. 3.20), dando origem ao "ponto de colocação não nodal" c ao "elemento de colocação não nodal" (FERREIRA, 1990). Este lipo de aplicação é utilizado para superar eventuais descontinuidades nos pontos nodais de elementos adjacentes a contornos com angulosidade.

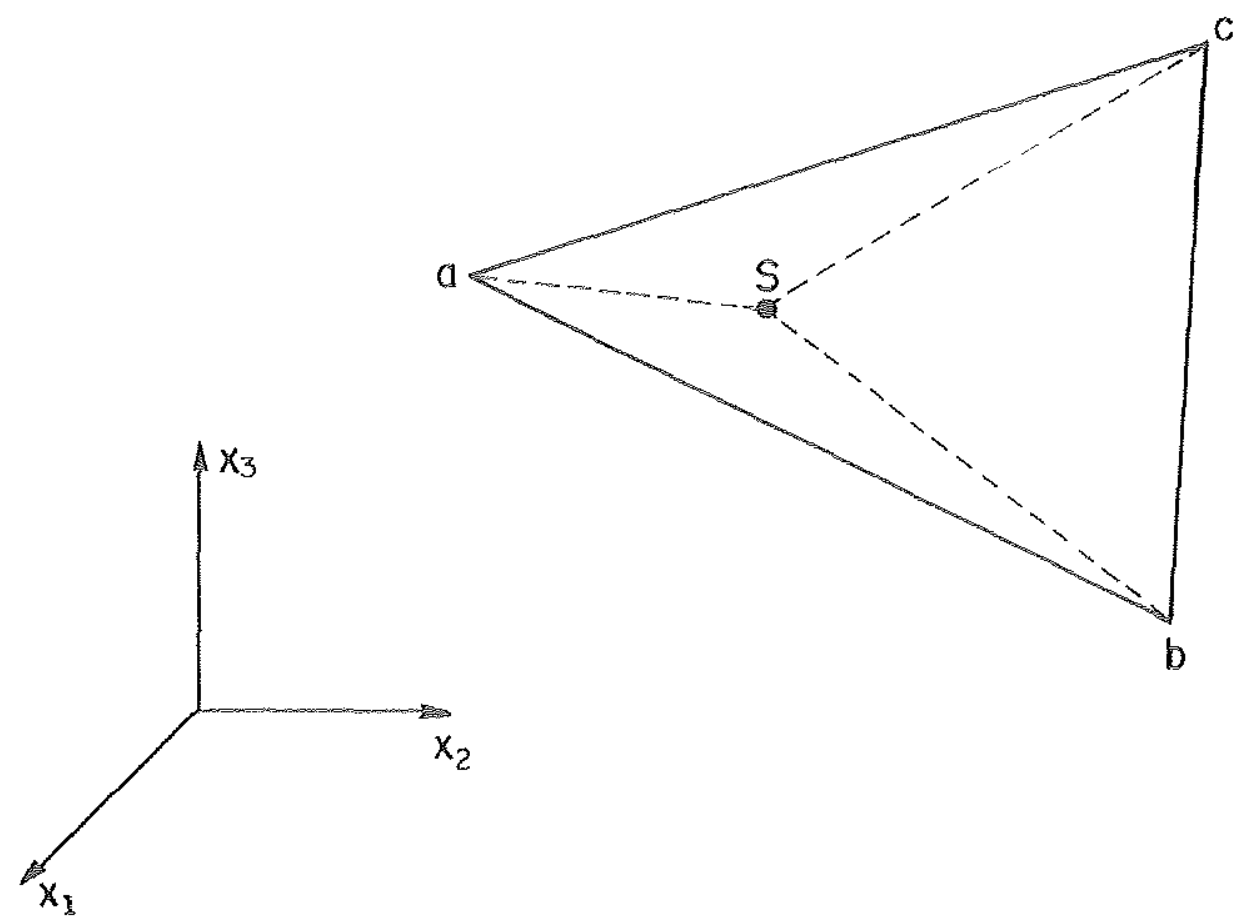

FIGURA 3.20 - Elcmento de colocação não nodal.

Ao aplicar tal procedimento ter-sc-ão vários nós no contorno com as mesmas coordenadas, porém com cada um pertencendo a clementos distintos, dando lugar ao que se denomina nó múltiplo. 


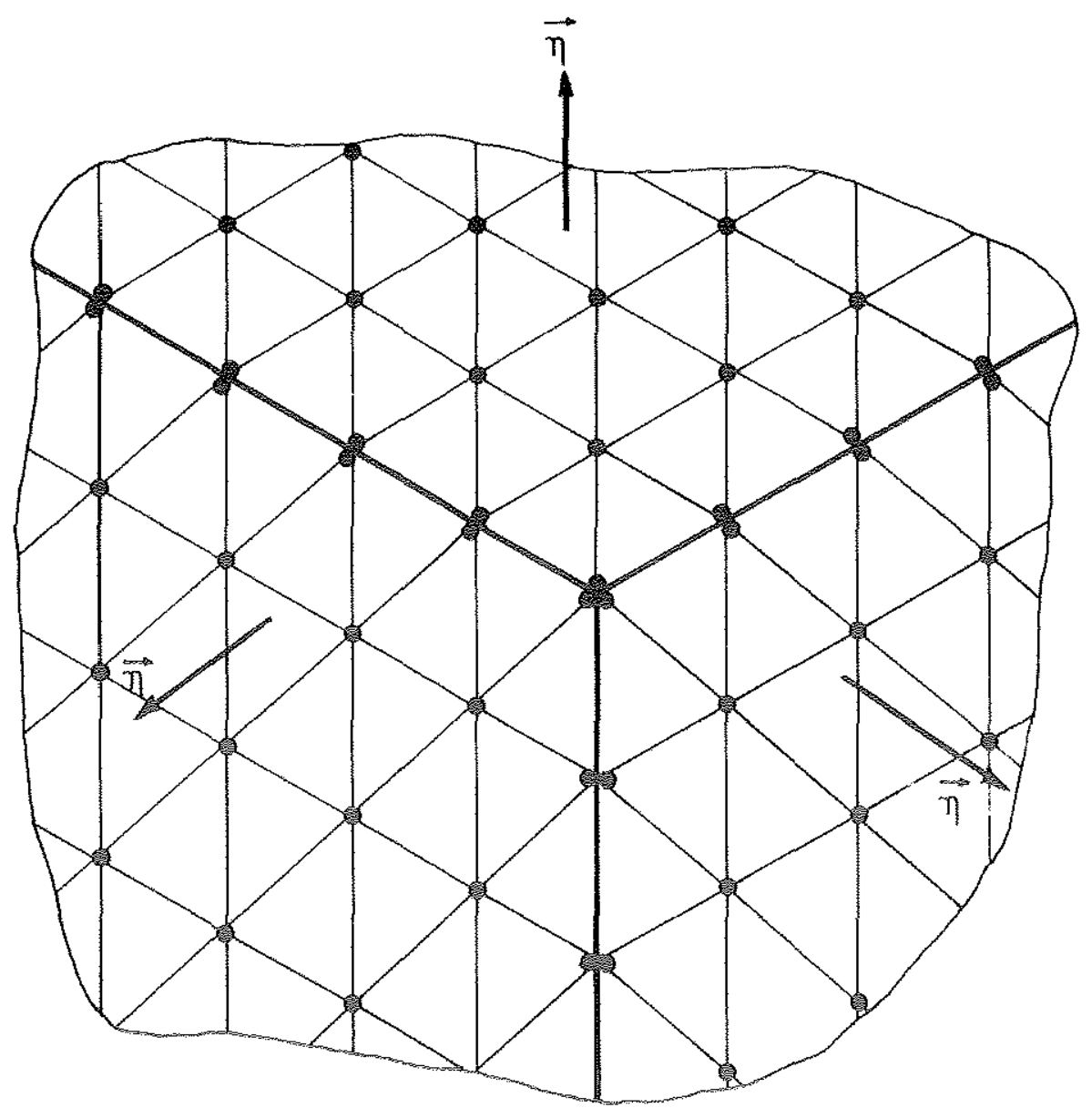

FIGURA 3.21 - Representação de nós múltiplos.

Após a integração do clemento de colocação não nodal, que corresponde à integração singular, e dos demais elementos (integração não singular), os deslocamentos do ponto de colocação não nodal são interpolados em função dos valores nodais siluados nos vértices do elemento, por meio de funções de interpolaçào, como:

$$
\underset{\sim}{u}(\mathbb{S})=\underset{\sim}{\Phi^{T}}(\mathbb{S}){\underset{\sim}{U}}^{N}(\mathbb{Q})
$$

Admitindo-se que $\mathbf{a}, \mathbf{b}$ e $\mathbf{c}$ sejam os pontos nodais de um elemento (Fig. 3.20), a equação (3.108) explicitada, toma a seguinte forma: 


$$
\left\{\begin{array}{l}
u_{1}(S) \\
u_{2}(S) \\
u_{3}(S)
\end{array}\right\}=\left[\begin{array}{ccccccccc}
\phi^{1} & 0 & 0 & \phi^{2} & 0 & 0 & \phi^{3} & 0 & 0 \\
0 & \phi^{1} & 0 & 0 & \phi^{2} & 0 & 0 & \phi^{3} & 0 \\
0 & 0 & \phi^{1} & 0 & 0 & \phi^{2} & 0 & 0 & \phi^{3}
\end{array}\right]\left\{\begin{array}{c}
U^{a} \\
\tilde{U}^{b} \\
\tilde{U}^{b} \\
U^{\mathfrak{c}}
\end{array}\right\}
$$

A cquação (3.107), escrita explicitamente para o ponto $\mathbf{S}$ situado no interior do elemento c relacionado ao ponto nodal "a" deste, fica:

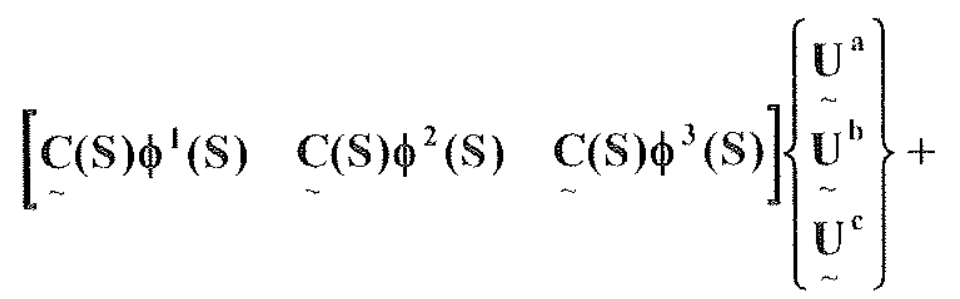

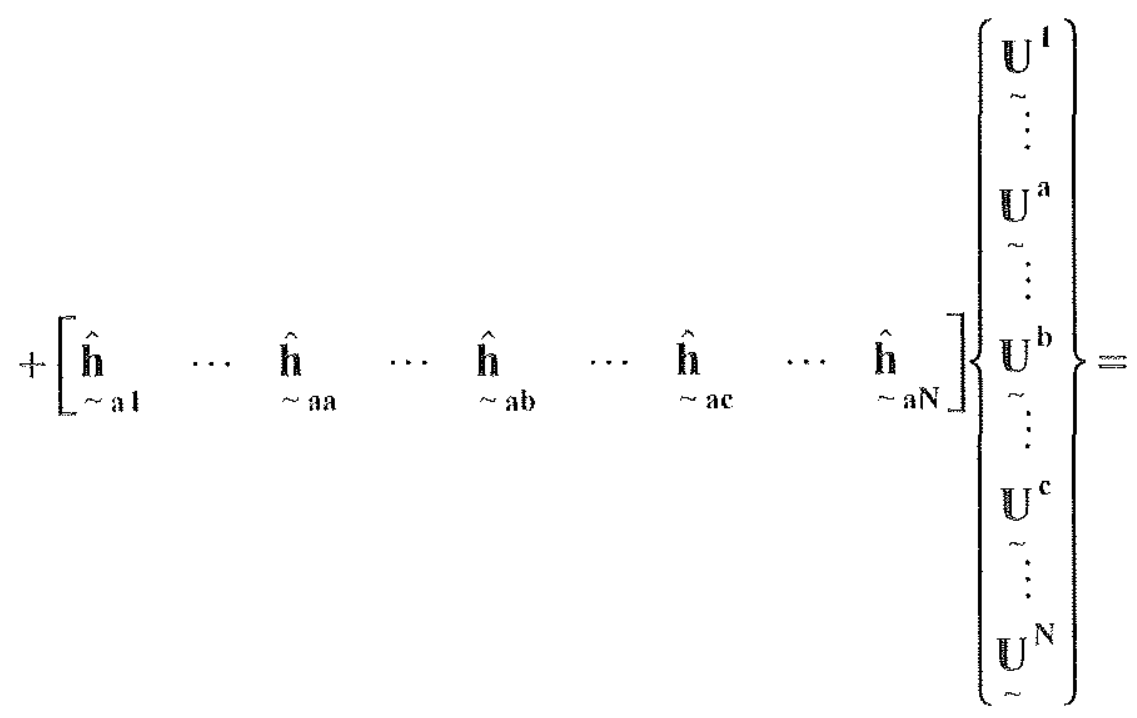

$$
\begin{aligned}
& =\left[\begin{array}{ccccccccc}
g & \cdots & g & \cdots & g & \cdots & g & \cdots & g \\
\sim \sim a 1 & & \sim a a & & \sim a b & & \sim a c & & \sim a N
\end{array}\right]\left\{\begin{array}{c}
\mathbb{P}^{1} \\
\sim \\
\vdots \\
\mathbb{P}^{a} \\
\sim \\
\mathbb{P}^{b} \\
\sim \\
\vdots \\
\mathbb{P}^{\mathrm{c}} \\
\sim \\
\vdots \\
\mathbb{P}^{N} \\
\sim
\end{array}\right\}
\end{aligned}
$$


Somando-se os correspondentes coeficientes relativos aos deslocamentos $\mathbf{U}^{\mathrm{a}}, \mathbf{U}^{\mathbf{b}}$ c $\mathbf{U}^{\mathrm{c}}$, obtêm-sc:

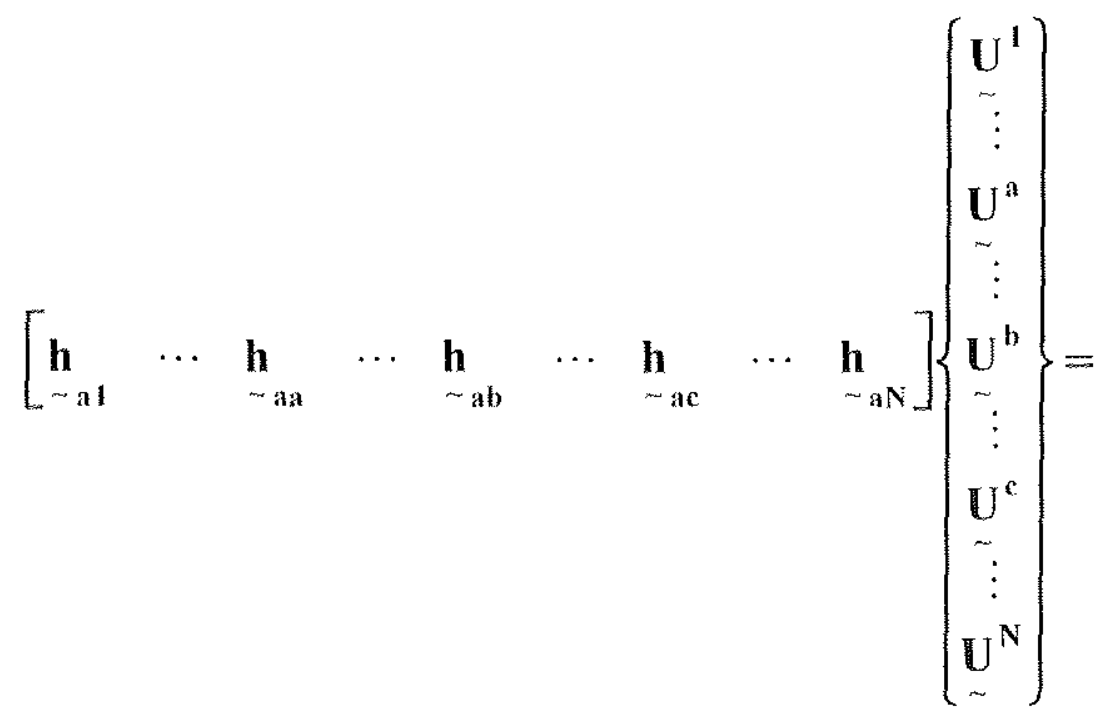

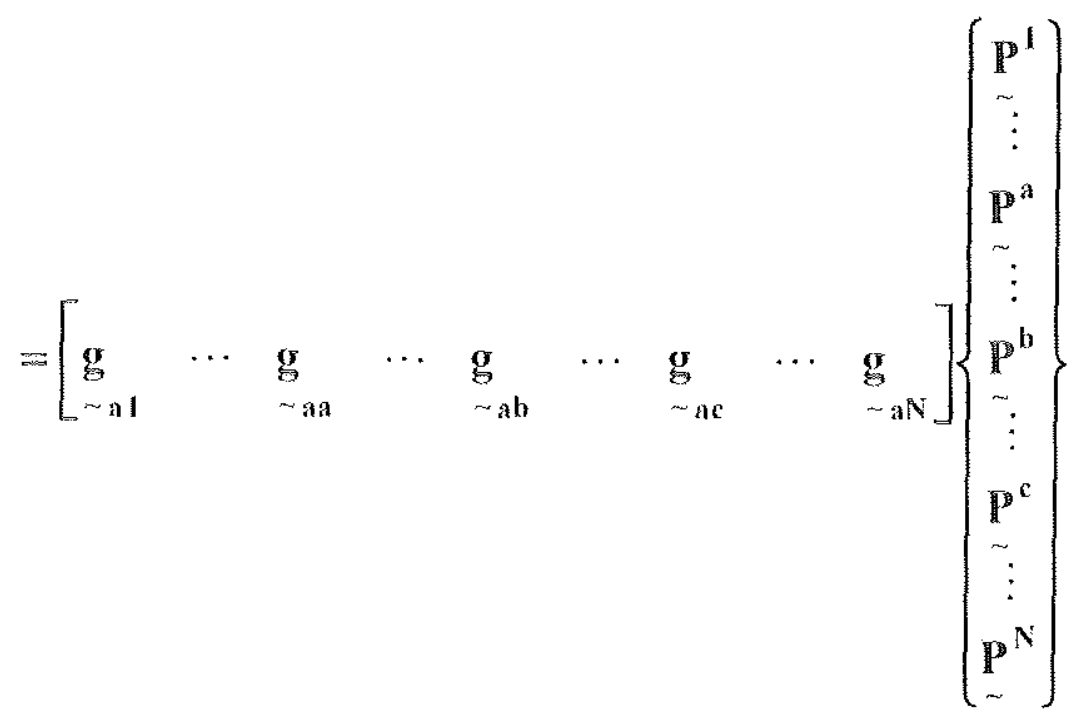

sendo:

$$
\begin{aligned}
& \underset{\sim a}{\mathbf{h}}=\hat{\mathbf{h}}+\underset{\sim a a}{\mathbb{C}(S) \phi^{\prime}(S)} \\
& \underset{\sim a b}{\mathbf{h}}=\hat{\sim} \hat{\mathbf{h}}+\underset{\sim}{\mathbb{C}(S) \phi^{2}(S)} \\
& \underset{\sim a c}{\mathbf{h}}=\hat{\mathbf{h}}_{\sim a c}+\mathbb{C}(S) \phi^{3}(S)
\end{aligned}
$$

As equações (3.110) c (3.111) apresentadas na forma matricial para todos os pontos nodais, respectivamente, tomam a seguintes formas: 


\section{$\mathbf{C} \Phi \mathbf{U}+\hat{\mathbf{H}} \mathbf{U}=\mathbf{G P}$}

\section{$\mathbf{H} \mathbf{U}=\mathbf{G} \mathbf{P}$}

Aplicando-se as condições de contorno à equação $(3.116)$ e efetuando-se as trocas de colunas das matrizes $\mathbf{H}$ e $\mathbf{G}$, de forma que todas as incógnitas fiquem no primeiro membro, será obtido um sistema de equações algébricas semelhante ao dado pela equação (3.11), cuja solução fornecerá as incógnitas do problema.

A utilização do elemento de colocação não nodal em elementos adjacentes a descontinuidades (elemento de canto) facilita a montagem do sistema de equações, $\mathrm{cm}$ razão do ponto fonte situar-se no interior do elemento, fïcando sempre $\mathrm{em}$ um contorno sem angulosidades; portanto, os coeficicntes não nulos da matriz $\mathbb{C}$ valcrão 0,5, isto $\mathrm{e}:$

$$
\underset{\sim}{\mathbb{C}}(\mathbb{S})=\left[\begin{array}{ccc}
0,5 & 0 & 0 \\
0 & 0,5 & 0 \\
0 & 0 & 0,5
\end{array}\right]
$$

Caso a cquação (3.118) seja aplicada em pontos nodais de um clemento de canto. as submatrizes $\mathbf{h}$ da diagonal principal da matriz $\mathbb{H}$ poderão ser calculadas indirctamente por movimento de corpo rígido, conforme NAKAGUMA (1979). Assim, para um ponto nodal "a", tem-se;

$$
\underset{\sim a \mathbf{a}}{\mathbf{h}}=-\sum_{\mathbf{j}=\mathbf{1}}^{N} \hat{\mathbf{h}} \underset{\sim_{a j}}{ }(\mathbf{j} \neq \mathbf{a})
$$

Esta expressão é válida para regiões finitas. Para regiōes infinitas (solução fundamental de Kelvin) e semi-intinitas (soluções fundamentais de Mindlin e Boussincsq-Cerruti), tem-se:

$$
\underset{\sim \text { aa }}{\mathbf{h}}=-\sum_{\mathbf{j}=1}^{N} \hat{\sim_{\text {aj }}} \hat{\mathbf{h}}+\mathbb{I}(\mathbf{j} \neq \mathbf{a})
$$


Neste trabalho, será utilizado o elemento linear contínuo em todos os clementos do contorno, exceto nos elementos de canto, no qual será empregado o elemento de colocação nào nodal.

\subsection{DESLOCAMENTOS EM PONTOS DO DOMÍNIO}

Os deslocamentos $\mathrm{em}$ pontos do domínio são determinados $\mathrm{em}$ função dos deslocamentos e forças de superfícic no contorno do sólido, como exposto anteriormente, utilizando-se a identidade de Somigliana aproximada (equação (2.29)), isto é:

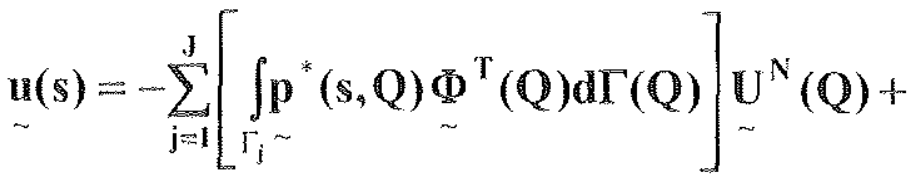

$$
\begin{aligned}
& +\sum_{j=1}^{J}\left[\int_{\Gamma_{j}} \mathrm{u}^{*}(s, Q) \Phi^{\mathrm{T}}(\mathrm{Q}) \mathrm{dT}(\mathrm{Q})\right] \mathrm{P}^{\mathrm{N}}(\mathrm{Q})+
\end{aligned}
$$

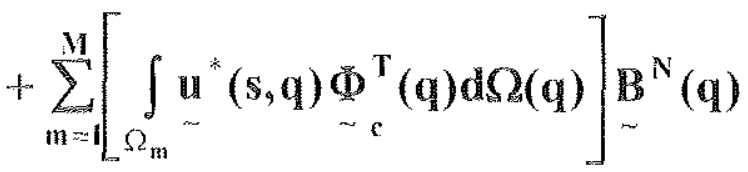

O ponto lonte pertence, agora, ao domínio c, portanto, todas a integrais de contorno scrão calculadas numericamente como integrais não singulares. As forças volumétricas, como dito, nào serão consideradas neste trabalho, não sendo, assim, necessário calcular as integrais de domínio correspondentes às mesmas. 


\subsection{APLICAÇÕES NUMÉRICAS}

Neste item, serão apresentadas algumas aplicações do Método dos Elementos de Contomo para sólidos tridimensionais, envolvendo as soluçŏes fundamentais de Mindlin e Boussinesq-Cerruti.

\subsubsection{EXEMPLO 1 - Área circular uniformemente carregada}

Neste excmplo, como mostra a Fig. 3.22, uma área circular de raio igual a 1 $\mathrm{cm}$, situada na superfícic do meio semi-infinito, está submetida a uma carga uniformemente distribuída, de intensidade igual a $10 \mathrm{~N} / \mathrm{cm}^{2}$, módulo de elasticidade longitudinal de $10 \mathrm{kN} / \mathrm{cm}^{2} \mathrm{e}$ coeficiente de Poisson 0,3 .
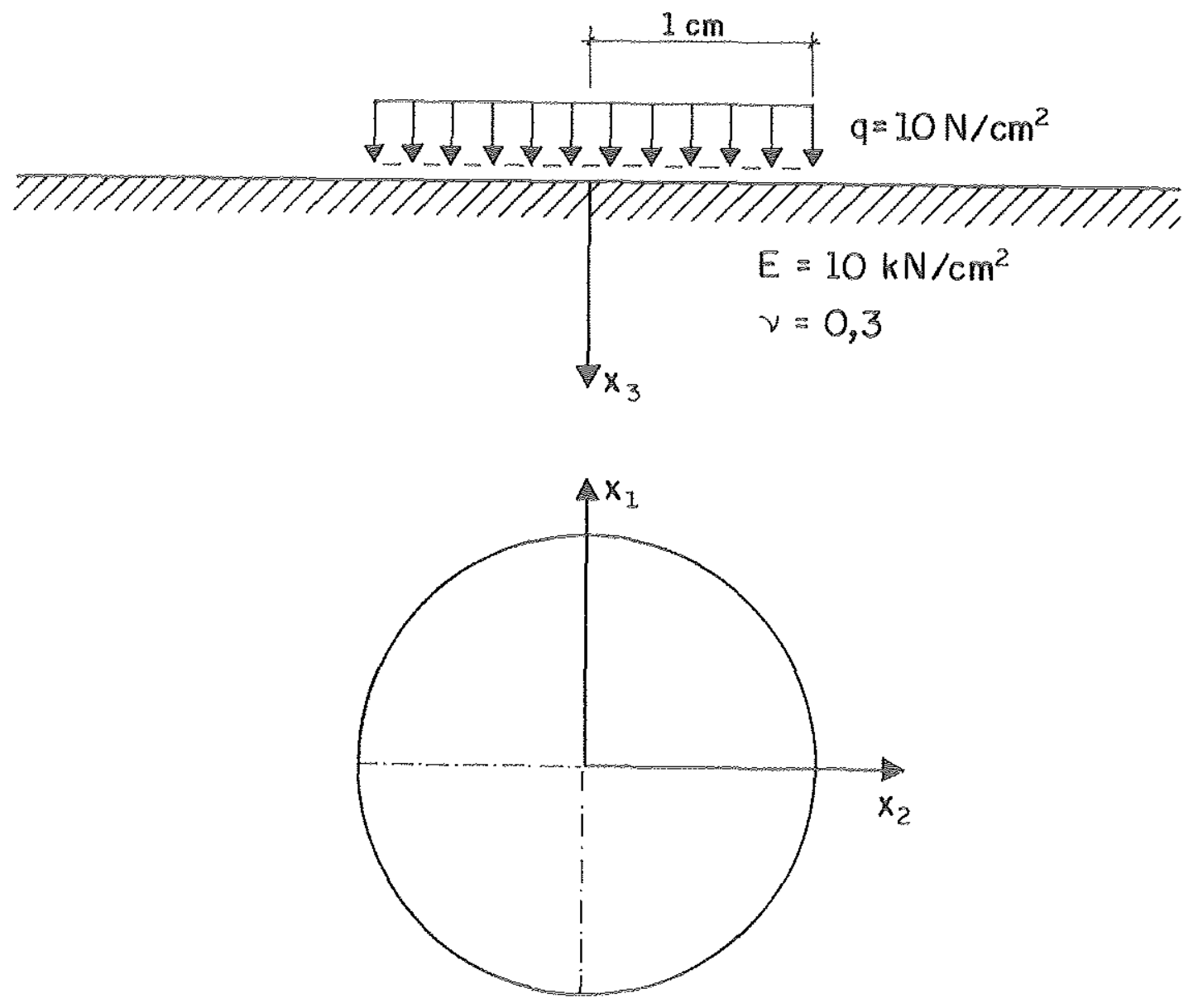

FIGURA 3.22 - Área circular uniformemente carregada. 
A Fig. 3.23 mostra a discretização da área circular através de elementos triangulares.

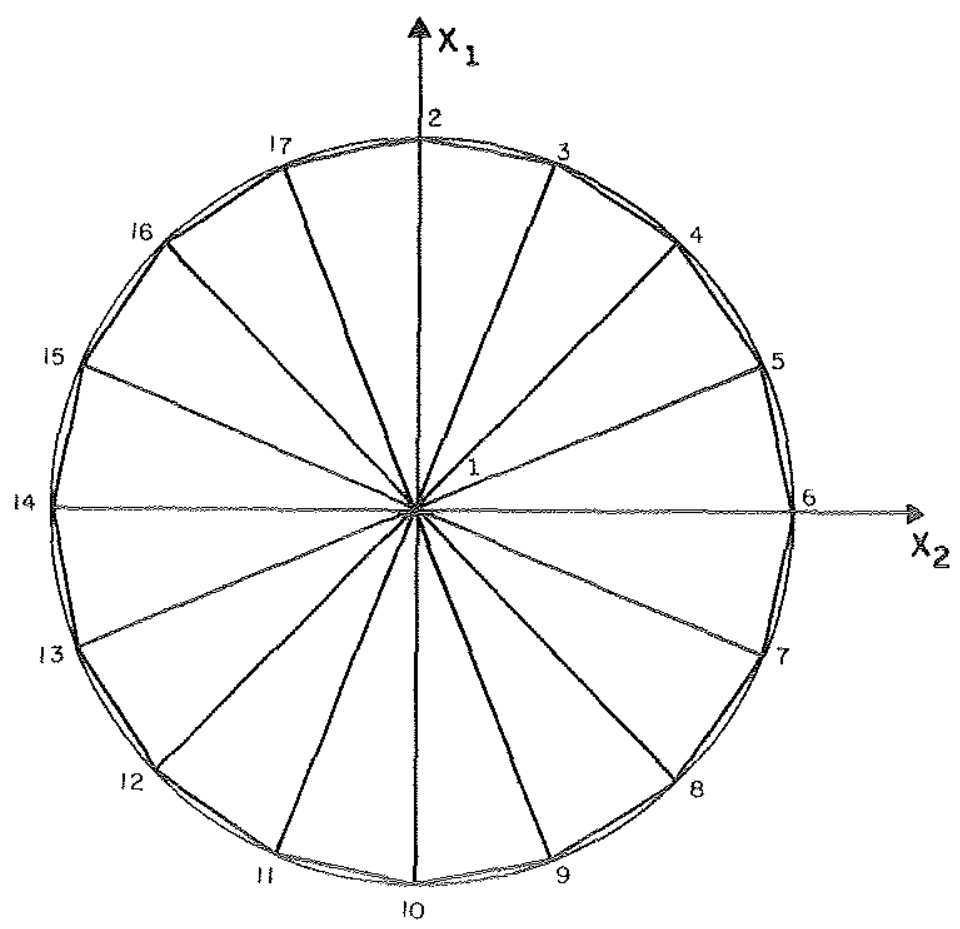

FIGURA 3.23 - Discretização da área circular carregada.

A solução exata deste problema é apresentada em TIMOSHENKO \& GOODIER (1975) e scrá comparada aos resultados obtidos, utilizando-se a solução fundamental de Mindlin, com a área circular sendo discretizada $\mathrm{em}$ dezesseis elementos (Fig. 3.23) e trinta e seis clementos e apresentados nas tabelas e gráficos a seguir. 
TABELA 3.1 - Valores correspondentes aos deslocamentos verticais obtidos a partir da solução exata e numérica.

\begin{tabular}{|c|c|c|c|}
\hline \multirow{2}{*}{$\begin{array}{c}x_{1} \\
(\mathrm{~cm})\end{array}$} & \multirow{2}{*}{$\begin{array}{l}\text { Solução Exata } \\
\qquad\left(\times 10^{-4} \mathrm{~cm}\right)\end{array}$} & \multicolumn{2}{|c|}{ Solução de Mindlin } \\
\hline & & $\begin{array}{l}16 \text { elementos } \\
\left(\times 10^{-4} \mathrm{~cm}\right)\end{array}$ & $\begin{array}{c}36 \text { elementos } \\
\left(\times 10^{-4} \mathrm{~cm}\right)\end{array}$ \\
\hline 0,00 & 18,2000 & 17,966 & 18,154 \\
\hline 0.25 & 17,9122 & 17.619 & 17,802 \\
\hline 0,50 & 17,0027 & 17,152 & 17,153 \\
\hline 0,75 & 15,2764 & 14,711 & 14,967 \\
\hline 1,00 & 11,5865 & 11,147 & 11,455 \\
\hline 2,00 & 4,7076 & 4,535 & 4,634 \\
\hline 3,00 & 3,0773 & 2,966 & 3,029 \\
\hline 4,00 & 2,2932 & 2,211 & 2,258 \\
\hline 5,00 & 1,8290 & 1,764 & 1,801 \\
\hline 6,00 & 1,5220 & 1,468 & 1,498 \\
\hline 7,00 & 1,3030 & 1,257 & 1,283 \\
\hline 8,00 & 1,1400 & 1,099 & 1.122 \\
\hline 9,00 & 1,0127 & 0,977 & 0,997 \\
\hline 10,00 & 0,9111 & 0,879 & 0,897 \\
\hline
\end{tabular}

As Fig. 3.24 c 3.25 mostram os deslocamentos verticais ao longo do eixo $x_{1}$ e do eixo $x_{3}$, respectivamente, obtidos a partir das soluções fundamentais de Mindlin, ao quais são comparados com os valores exatos (TIMOSHENKO\&GOODIER, 1975). 


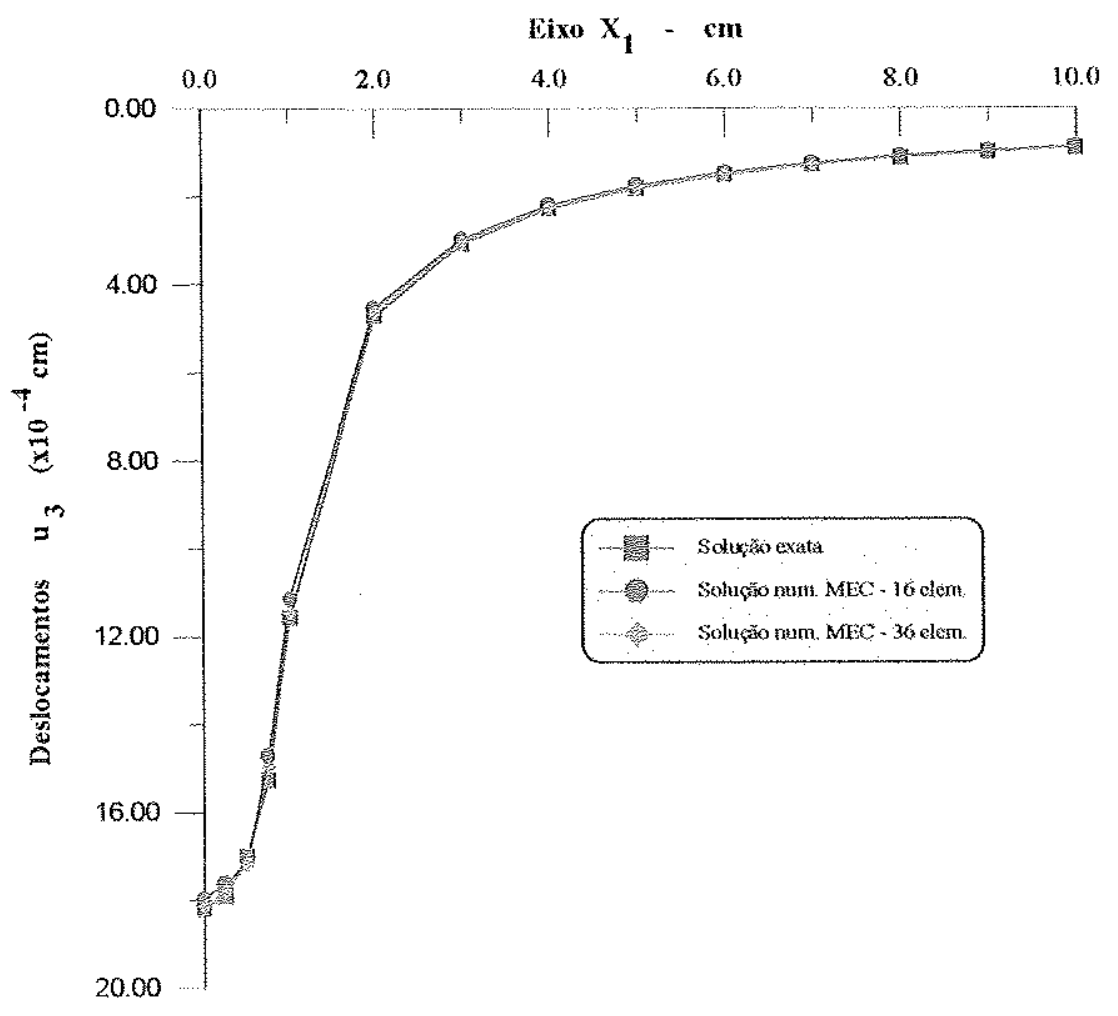

FIGURA 3.24 - Deslocamentos verticais ao longo do eixo $\mathbf{x}_{\mathbf{1}}$.

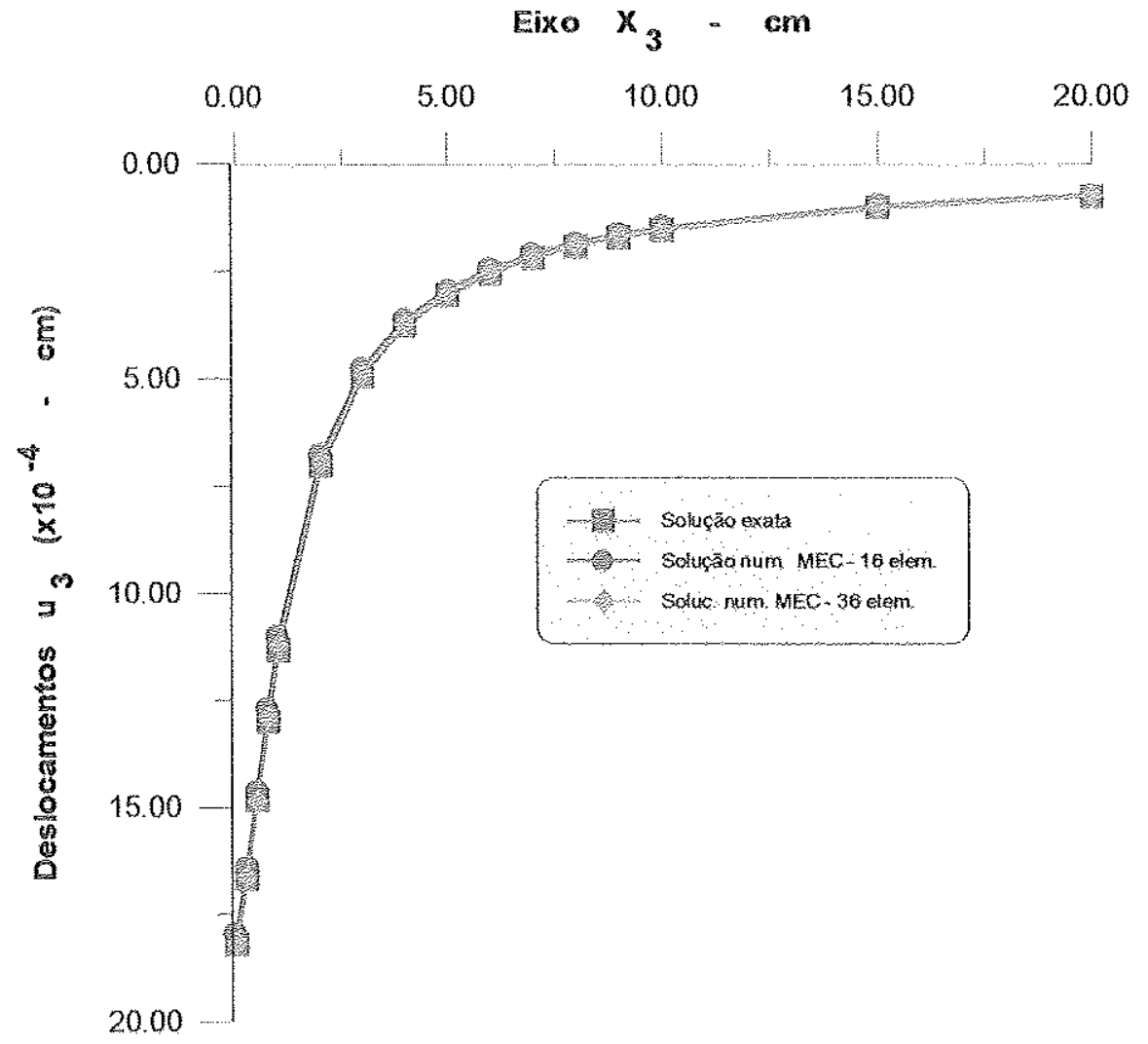

FIGURA 3.25 - Deslocamentos verticais ao longo do eixo $\mathbf{x}_{3}$. 


\subsubsection{EXEMPLO 2 - Área retangular uniformemente carregada}

Neste exemplo, apresentado na Fig. 3.26, analisa-se uma área retangular na superficie do espaço semi-intinito, submetida a um carregamento uniforme igual a $95,76 \mathrm{~N} / \mathrm{m}^{2}$. O solo apresenta módulo de clasticidade longitudinal igual a $44,48 \mathrm{kN} / \mathrm{m}^{2}$ e coeficiente de Poisson 0,3 .
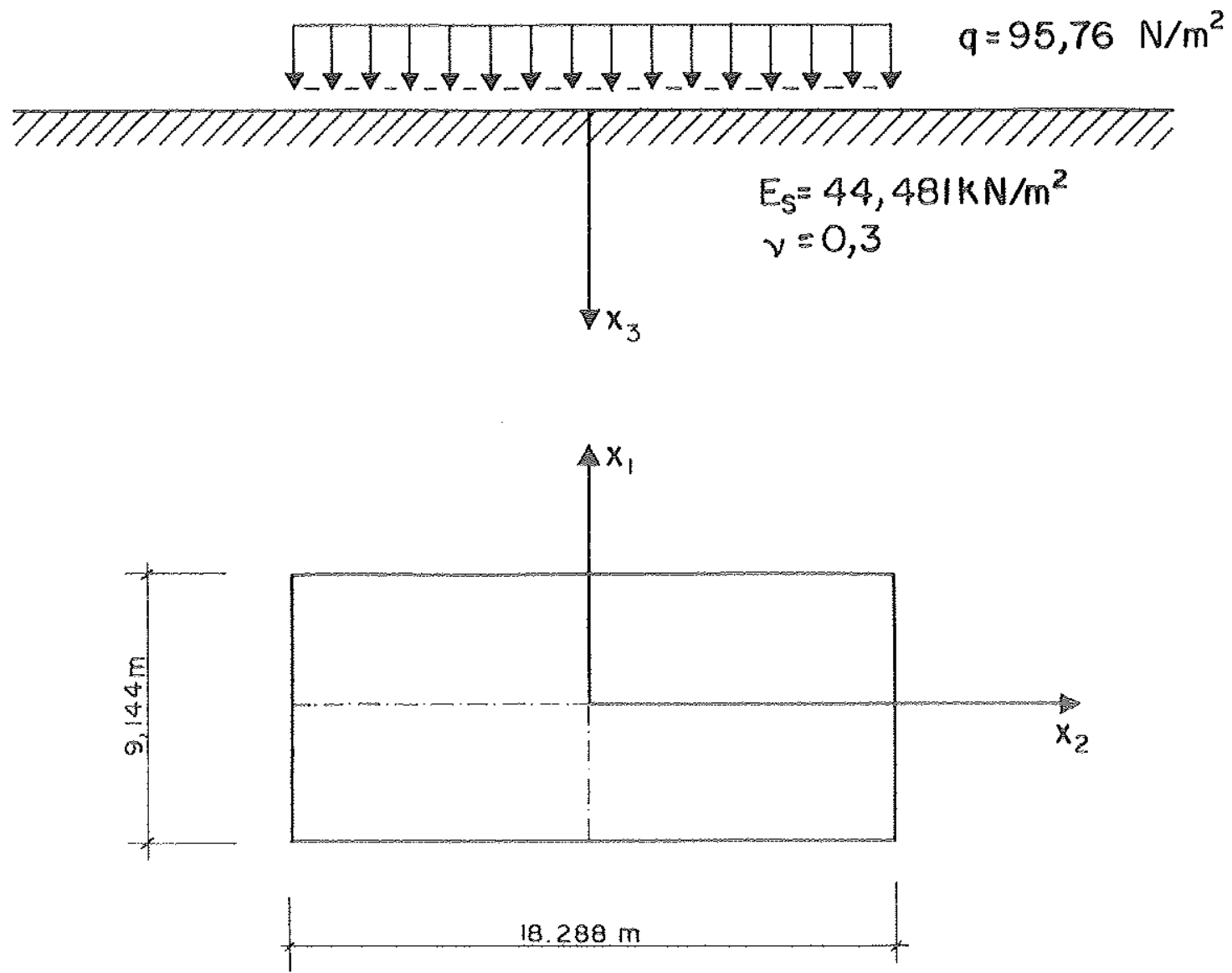

FIGURA 3.26 - Área retangular uniformemente carregada.

A Fig. 3.27 mostra a discretização da área retangular en trinta e scis clementos triangulares lineares, que representam exatamente a área carregada, não existindo qualquer aproximação quanto à geometria. 


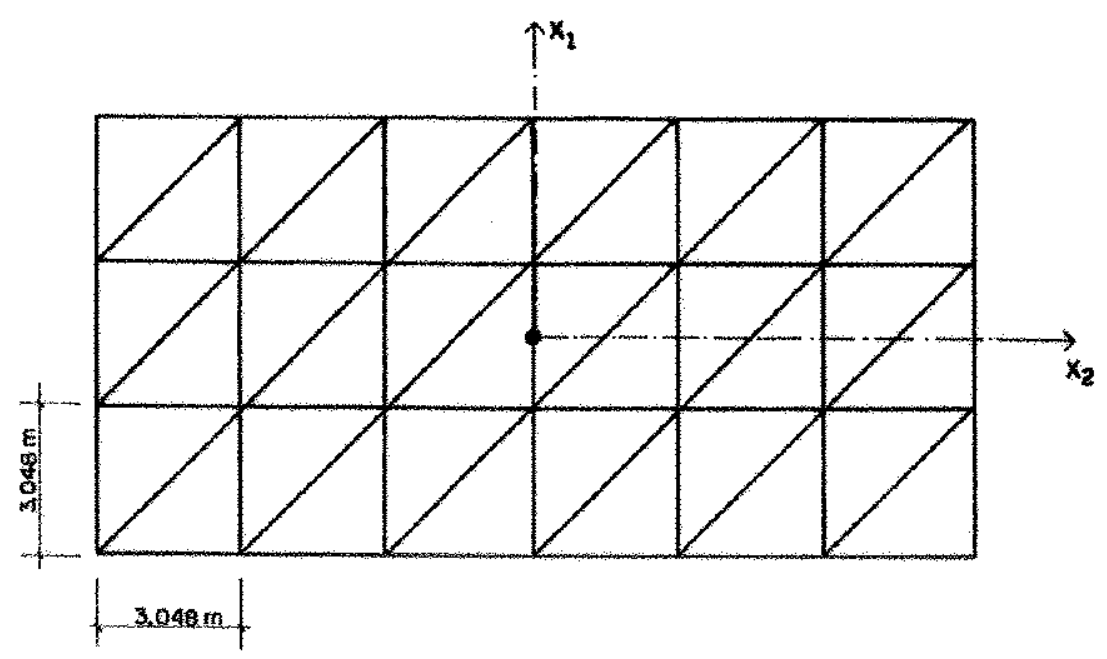

FIGURA 3.27 - Discretização da área retangular carregada.

Os resultados foram obtidos utilizando-se as soluções fundamentais de Mindlin e comparadas com os valores exatos obtidos a partir (TIMOSHENKO\&GOODIER, 1975). São determinados os deslocamentos verticais na superfície do meio contínuo, ao longo do eixo $x_{1}$ e do eixo $x_{2}$, mostrados nas Fig. 3.28 e 3.29 , respectivamente.

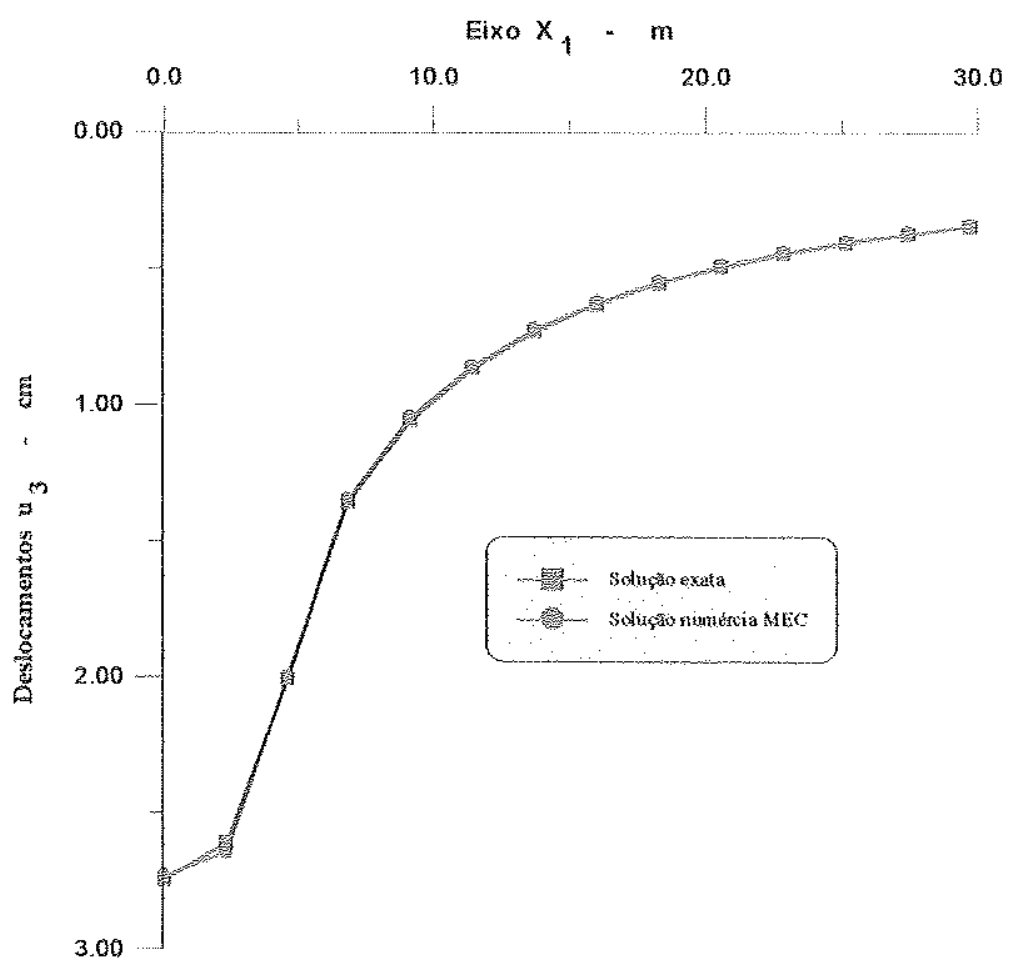

FIGURA 3.28 - Deslocamentos verticais ao longo do eixo $\mathrm{x}_{\mathbf{1}}$. 


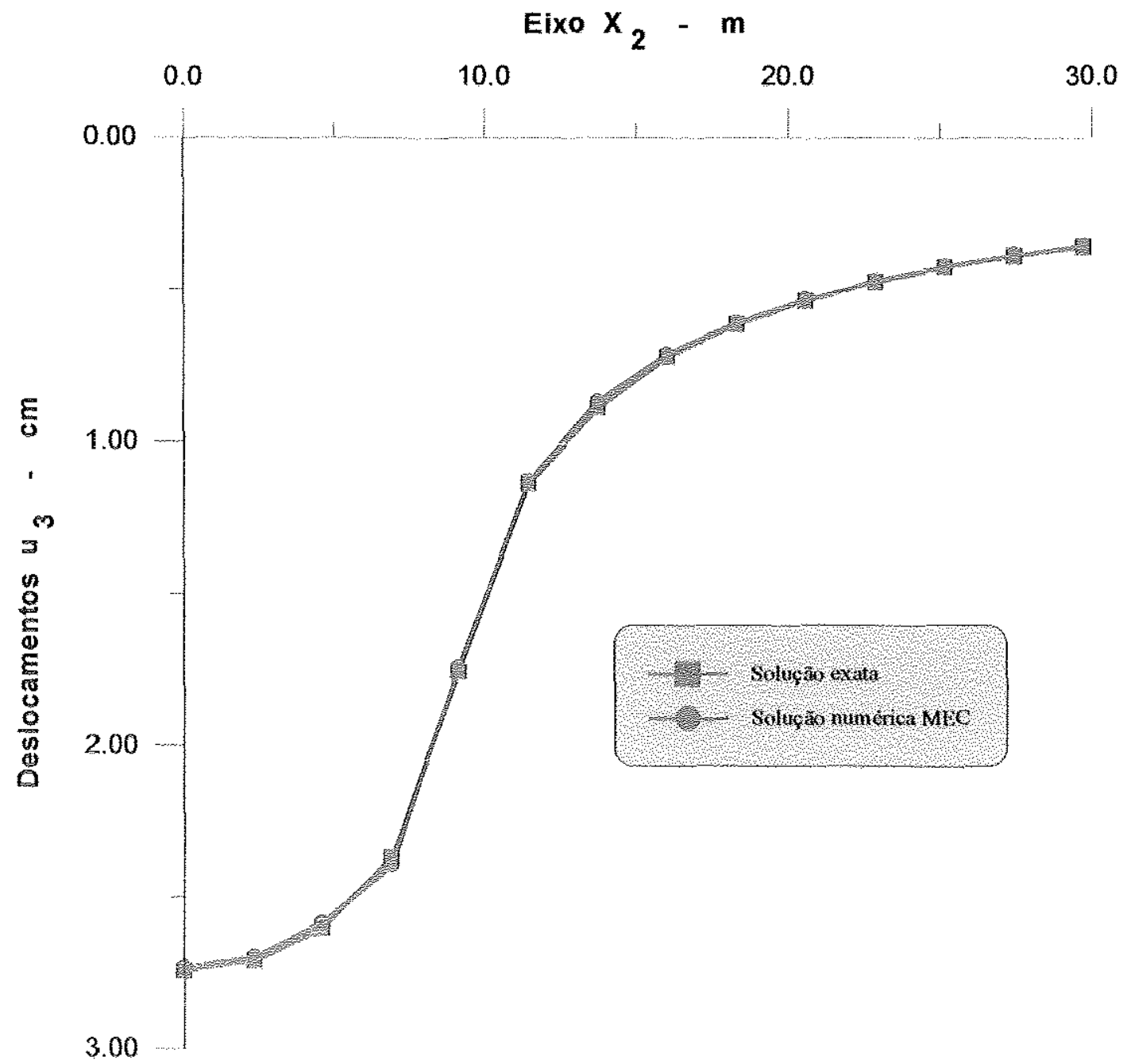

FIGURA 3.29-Deslocamentos verticais ao longo do eixo $\mathbf{x}_{2}$.

Os deslocamentos verticais ao longo do eixo $x_{3}$ e de um eixo também vertical que passa por um dos vértices da área retangular, são mostrados nas Fig. 3.30 e 3.31, respectivamente. 


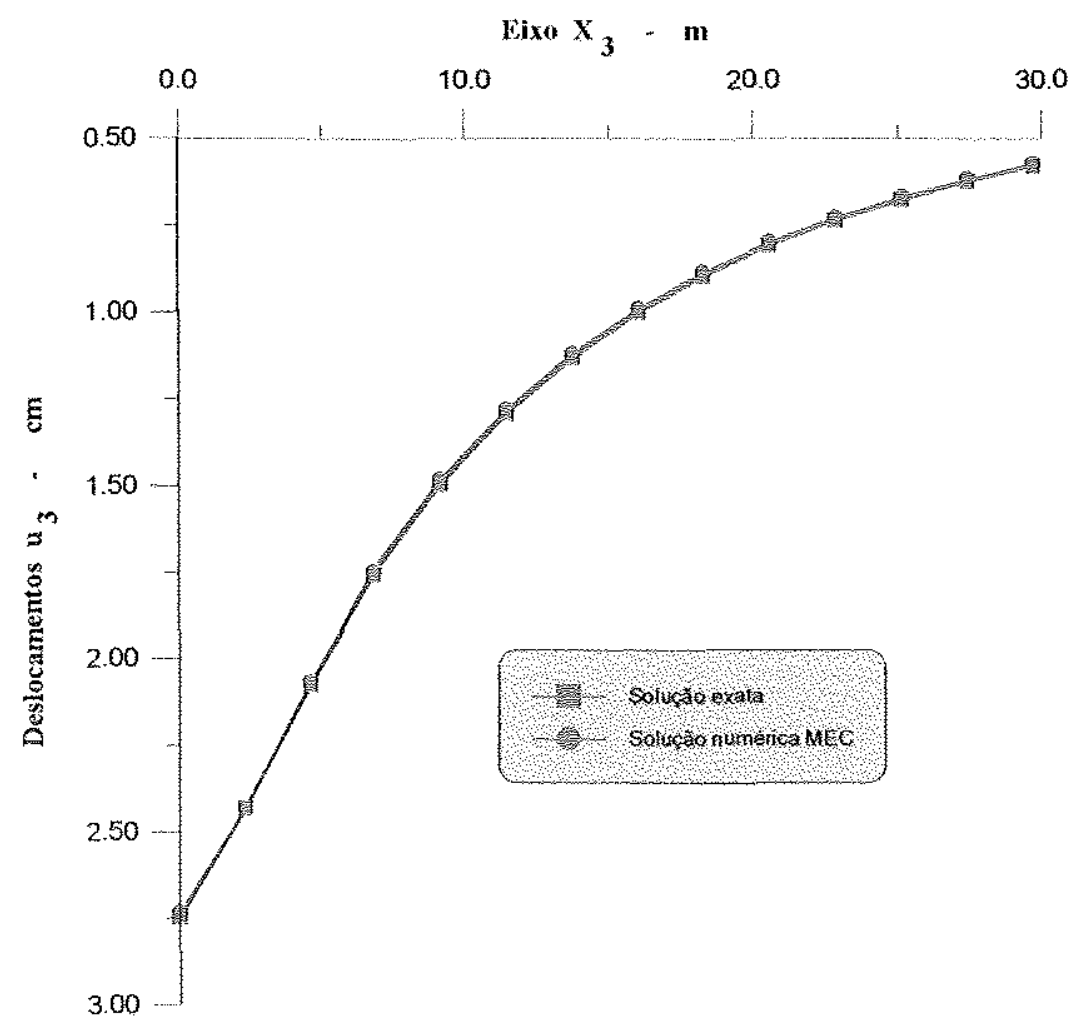

FIGURA 3.30 - Deslocamentos verticais ao longo do eixo $\mathbf{x}_{3}$.

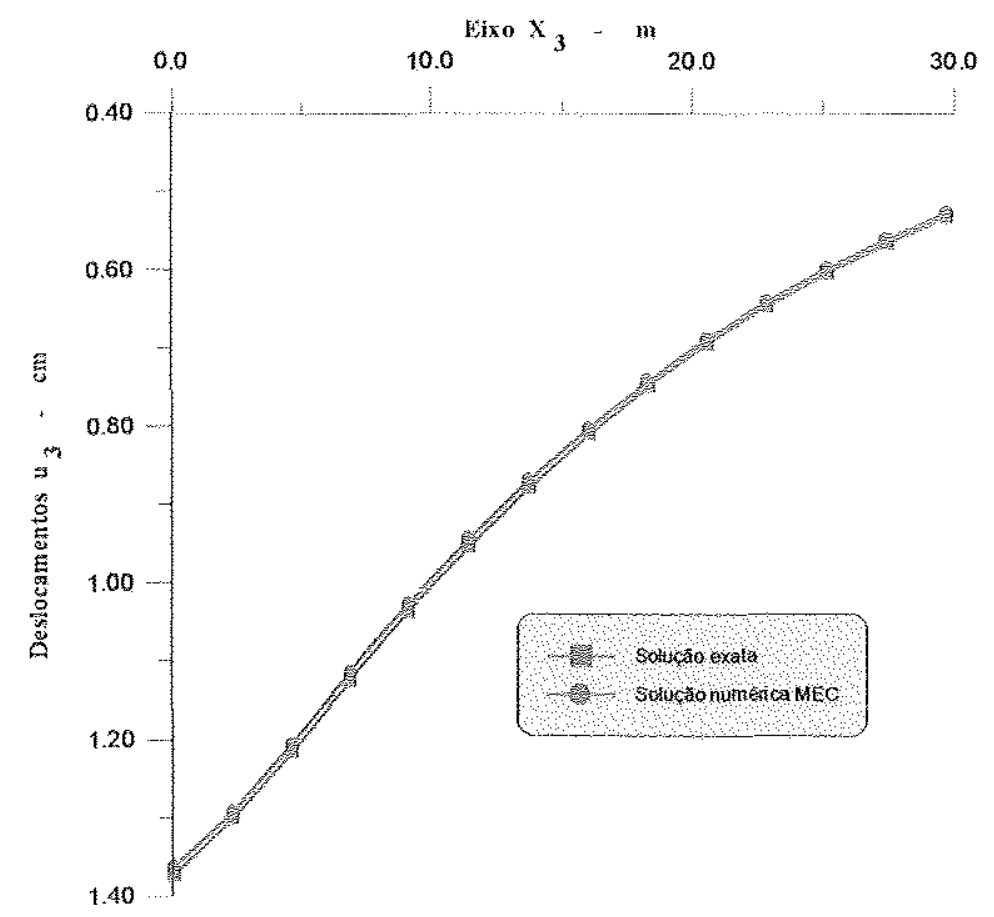

FIGURA 3.31 - Deslocamentos verticais ao longo de um eixo paralelo ao eixo $x_{3}$ que passa pelo vértice da área retangular. 


\section{A COMBINAÇÃO MEC-MEF}

\subsection{INTRODUÇÃO}

Neste capítulo apresenta-se um modelo para a determinação da matriz de rigidez de fundações em estacas, considerando-se a interação solo-fundação. O objetivo é de adaptá-lo a um programa de elementos finitos, para o cálculo de estruturas de edificios, seguindo a linha proposta por RAMALHO (1990), que define uma matriz de rigidez para sapatas rígidas, utilizando o Método dos Elementos de Contorno. A idealização do modelo, tem como característica principal a combinação do Método dos Elementos de Contorno com o Método dos Elementos Finitos. Todas as etapas envolvidas nessa formulação, serão detalhadas nos próximos itens deste capítulo.

\subsection{INTERACÃO DE UM MEIO TRIDIMENSIONAL COM ESTRUTURAS DE BARRAS}

No caso do problema a ser equacionado, o de estacas cravadas no meio contínuo, é necessário incluir no segundo membro da equação (2.34), o termo adicional correspondente à aplicação de uma carga distribuída ao longo das estacas, no sistema global de referência, conforme VENTURINI (1988), e como mostrado na Figura 4.1. A dedução de tal termo, pode ser feita, por exemplo, a partir do limite da integral de domínio das forças volumétricas. 


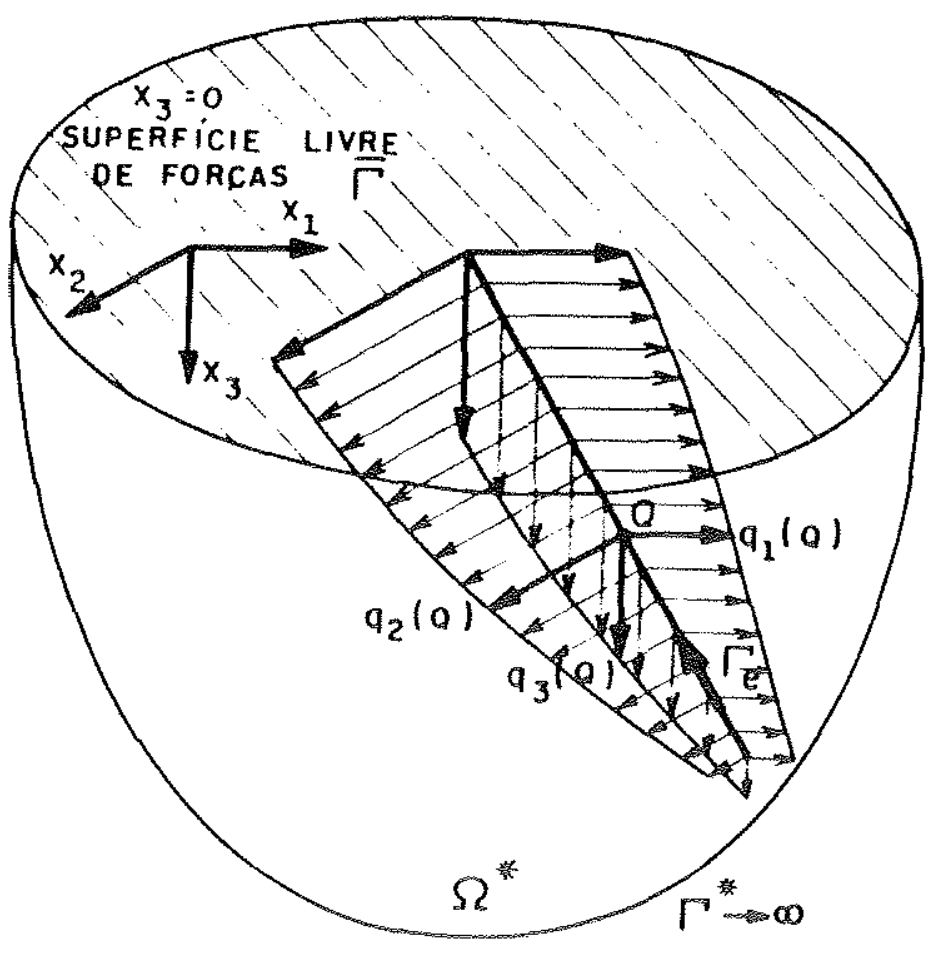

FIGURA 4.1 - Domínio tridimensional com linha de carga.

Para um domínio com $\mathbb{N}_{\mathbf{b}}$ linhas de carga, correspondentes às estacas, a equação de deslocamentos $(2.34)$ pode ser agora modificada chegando-se a seguinte forma:

$$
\begin{aligned}
& \mathrm{C}_{\mathrm{ij}}(\mathrm{S}) \mathrm{H}_{\mathrm{j}}(\mathrm{S})=-\int_{\Gamma} \mathrm{P}_{\mathrm{ij}}^{*}(\mathrm{~S}, \mathrm{Q}) \mathrm{u}_{\mathrm{j}}(\mathrm{Q}) \mathrm{d} \Gamma(\mathrm{Q})+
\end{aligned}
$$

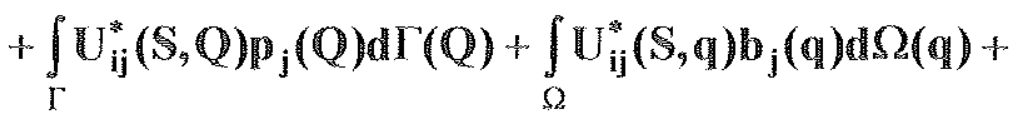

$$
\begin{aligned}
& +\sum_{b=1}^{N_{b}} \int_{\Gamma_{b}} U_{i j}^{*}(S, Q) q_{j}^{b}(Q) d \Gamma(Q)
\end{aligned}
$$

sendo, $\mathbf{q}_{\mathbf{j}}^{\mathbf{b}}(\mathbb{Q})$ representa as forças de interação aplicadas ao sólido tridimensional, $\mathbb{N}_{\mathbf{b}}$ é o número total de barras correspondentes às estacas e $\Gamma_{\mathbf{b}}$ as linhas onde essas forças estão aplicadas. Na eq. (4.1), a parcela correspondente as forças volumétricas pode ser negligenciada, para as aplicações a serem aqui desenvolvidas. Dessa forma, a equação integral de deslocamentos, pode ser escrita como: 


$$
\begin{aligned}
& C_{\mathrm{ij}}(S) \mathbf{u}_{\mathrm{j}}(S)=-\int_{\Gamma} P_{\mathrm{ij}}^{*}(S, Q) \mathbf{u}_{\mathrm{j}}(Q) \mathrm{d} \Gamma(Q)+ \\
& +\int_{\Gamma} U_{\mathrm{ij}}^{*}(S, Q) \mathbf{p}_{\mathbf{j}}(Q) \mathrm{d} \Gamma(Q)+\sum_{\mathrm{b}=1}^{N_{\Gamma_{\mathrm{e}}}} \int_{\mathrm{ij}}(S, Q) \mathbf{q}_{\mathrm{j}}^{\mathrm{b}}(Q) \mathrm{d} \Gamma(Q)
\end{aligned}
$$

\subsection{DISCRETIZAÇÃO DA EQUAÇÃO INTEGRAL DE CONTORNO}

A equação integral (4.2), pode ser transformada em uma representação algébrica, bastando para isso dividir a superfície de interação (ou de carga) em análise, por um número finito de elementos, e aproximar as variáveis reais do problema, utilizando-se valores nodais e funções de forma. Conhecidas as coordenadas dos pontos nodais de um elemento, qualquer ponto interno desse elemento, tem suas coordenadas representadas por:

$$
x_{i}=\psi^{j_{x}^{j}}
$$

sendo:

$\mathrm{x}_{\mathrm{i}}$, a coordenada de um ponto, referente ao eixo "i";

$\psi^{j}$, a função interpoladora;

$\mathbb{x}_{\mathfrak{i}}^{\mathbf{j}}$, a coordenada referente ao eixo "i" do ponto nodal "j".

A expressão (4.3), escrita na forma matricial, fica:

$$
=\psi^{\mathrm{T}} \mathrm{x}^{\mathrm{n}}
$$

onde $x$ é o vetor de coordenadas de um ponto, ou seja:

$$
\sim \sim \sim\left\{\begin{array}{l}
x_{1} \\
x_{2} \\
x_{3}
\end{array}\right\}
$$


De forma análoga, as variáveis do contorno, são aproximadas através de funções interpoladoras, expressas matricialmente como:

$$
\begin{aligned}
& \mathbf{u}=\phi^{\mathrm{T}} \underline{\sim}^{\mathrm{n}} \\
& \mathbf{q}=\phi^{\mathrm{T}} \mathbf{Q}^{\mathrm{n}}
\end{aligned}
$$

sendo que,

u e $\mathbf{q}$, são os vetores que contém as componentes de deslocamentos e de forças de interações, segundo os três eixos coordenados, respectivamente;

$\phi$, é a matriz das funções interpoladoras;

$\mathbf{U}^{\mathbf{n}}$ e $\mathbf{Q}^{\mathbf{n}}$, os vetores que contém os valores nodais de deslocamentos e forças de interação.

Exceto a superficie do meio contínuo, o contorno é dividido em " $\mathrm{M}$ " elementos planos. As linhas internas são divididas em " $\mathrm{J}$ " elementos unidimensionais e " $\mathrm{N}$ " pontos nodais e as forças de interação, como mostrado acima aproximadas por polinômios. Assumindo aproximações também para deslocamentos e forças de superficie em todos os elementos após a discretização do contorno do sólido, a equação integral de deslocamentos (4.2), escrita para o nó "S" no contomo, agora discretizada é dada por:

$$
\begin{aligned}
& \underset{\sim}{C}(S) u(S)=\sum_{i=1}^{M}\left[\int_{I_{i}^{*}} \mathbb{P}^{*}(S, Q) \phi^{T}(Q) d \Gamma(Q)\right] U_{\sim}^{n}(Q)+ \\
& +\sum_{i=1}^{M}\left[\int_{\Gamma_{i}} U^{*}(S, Q) \phi^{T}(Q) d \Gamma(Q)\right] P^{n}(Q)+ \\
& \sum_{j=1}^{J}\left[\int_{\Gamma_{i}} U_{\sim}^{*}(S, Q) \phi^{T}(Q) d \Gamma(Q)\right] Q^{n}(Q)
\end{aligned}
$$

Aplicando-se a eq. (4.6) aos " $\mathrm{N}$ " pontos nodais do contorno do sólido, estabelece-se um sistema de equações, que escrito na forma matricial, resulta:

$$
\underset{\sim}{\mathrm{CU}}=-\underset{\sim}{\mathbf{H}} \mathrm{U}+\underset{\sim}{\mathrm{G}} \mathbf{P}+\underset{\sim}{\mathbf{R}} \mathbf{Q}
$$


sendo que, as matrizes $\underset{\sim}{\hat{\mathbf{H}}}$ e $\mathbf{G}$ resultam do cálculo das integrais da equação (4.6) e já foram tratadas detalhadamente no capítulo 3.

$$
\begin{aligned}
& \text { As matrizes } \underset{\sim}{\mathbf{C}} \mathbf{\text { H }} \underset{\sim}{\hat{H}} \text { da equação (4.7) são somadas, resultando: } \\
& \underset{\sim}{\mathbf{H}}=\underset{\sim}{\mathbf{G P}}+\mathbf{R} \mathbf{Q}
\end{aligned}
$$

sendo, $\mathbf{Q}$, o vetor que contém os valores das componentes nodais das forças de interação em todos os nós e $\mathbf{R}$ a matriz dos coeficientes de influência calculados a partir da integral correspondente na eq. (4.6), e explicitados na eq. (4.8).

Como as novas incógnitas $\mathbf{Q}$, são admitidas no domínio, equações adicionais precisam ser escritas selecionando apropriados pontos de colocação, isto é,

$$
\underline{\sim}=-\mathbb{\sim} \cdot \underset{\sim}{U}+\underset{\sim}{G} \cdot \mathbf{P}+\mathbb{R} \cdot \mathbb{Q}
$$

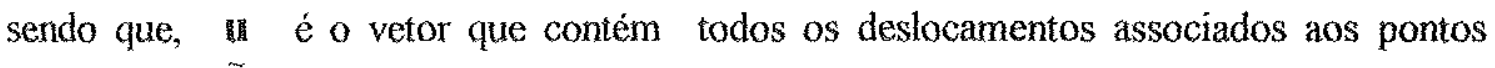
tomados ao longo das linhas internas.

Todas as matrizes na eq. (4.9) são calculadas de forma semelhante a eq. (4.6).

Considerando-se que, usualmente, apenas forças são prescritas para o problema sob consideração, a partir da eq. (4.8) pode-se escrever:

$$
U=\underset{\sim}{U^{0}+S Q}
$$

onde $\mathrm{U}^{0}$ representa os efeitos das forças prescritas e,

$$
S=\underset{\sim}{H^{-1}} \underset{\sim}{R}
$$

Substituindo-se, a expressão de $U$ dada na eq. (4.10) na eq. (4.9), tem-se:

$$
\mathbf{u}=\mathbf{u}^{0}+\mathbf{S} \mathbf{Q}
$$

em que,

$$
\mathbb{S}^{\prime}=\mathbb{R}^{\prime}-\underset{\sim}{ } \mathbf{H}^{\mathbf{H}} \mathbf{S}
$$

e,

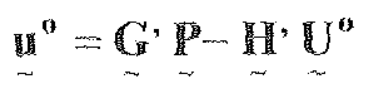


A eq. (4.12) relaciona todos os deslocamentos para pontos com forças de interação atuando ao longo das linhas internas (estacas).

\subsection{A COMBINAÇÃo DO MÉTOdo dOS ELEMENTOS DE CONTORNO COM O MÉTODO DOS ELEMENTOS FINITOS}

O método dos elementos de contorno, como já foi dito, tem-se mostrado bastante eficiente na modelagem de meios infinitos ou semi-infinitos. Esta característica é empregada na formulação desenvolvida, onde a superestrutura se apoia em blocos considerados rígidos. Tais blocos são utilizados, como elementos estruturais intermediários, para transferir as cargas da superestrutura para, as estacas, que por sua vez transferem seu carregamento ao meio semi-infinito.

A partir da formulação inicial apresentada, através do método dos elementos de contorno, consegue-se a interação entre o meio semi-infinito e as estacas que nele se encontram.

As estacas, por sua vez, são tratadas como elementos estruturais de barras dispostas no espaço tridimensional, pelo método dos elementos finitos, cujo sistema de equações lineares, que relaciona deslocamentos nodais, $\underset{\sim F}{U}$ e forças nodais equivalentes de interação $\underset{\sim F}{\mathbb{F}} \underset{\sim F}{\mathbb{F}^{0}}$ é o vetor independente de forças prescritas, pode ser escrito como:

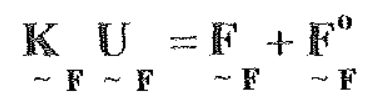

onde $\underset{\sim}{\mathbb{K}}$ é a matriz de rigidez do conjunto de estacas referida ao sistema global de referência.

No vetor $\underset{\sim}{U}$, são considerados os deslocamentos segundo as três direçôes coordenadas e as rotações em torno dos eixos $x_{1}$ e $\mathbb{x}_{2}$. Desta forma, o sistema de equações (4.16) é de ordem $5 \mathrm{~N}$, onde $\mathrm{N}$ é número de pontos nodais das estacas. Para se considerar a interação meio contínuo-elementos de barras, emprega-se uma formulação 
que permite a combinação do método dos elementos de contorno com o método dos elementos finitos. Vários autores têm-se dedicado a estudar esse assunto, dentre eles podemos citar: BREBBIA \& GEORGIOU (1979), BEER \& MEEK (1981), VALLABHAN \& SIVAKUMAR (1986), dentre outros, como foi apresentado no Capítulo 1.

Para apresentar a técnica da combinação, considera-se o domínio da Fig. 4.2, onde a região $\Omega_{1}$ está discretizada em elementos de contorno e a região $\Omega_{2}$ é tratada via elementos finitos, sendo que entre as duas regiões é definida a interface $\Gamma_{\mathbf{i}}$.

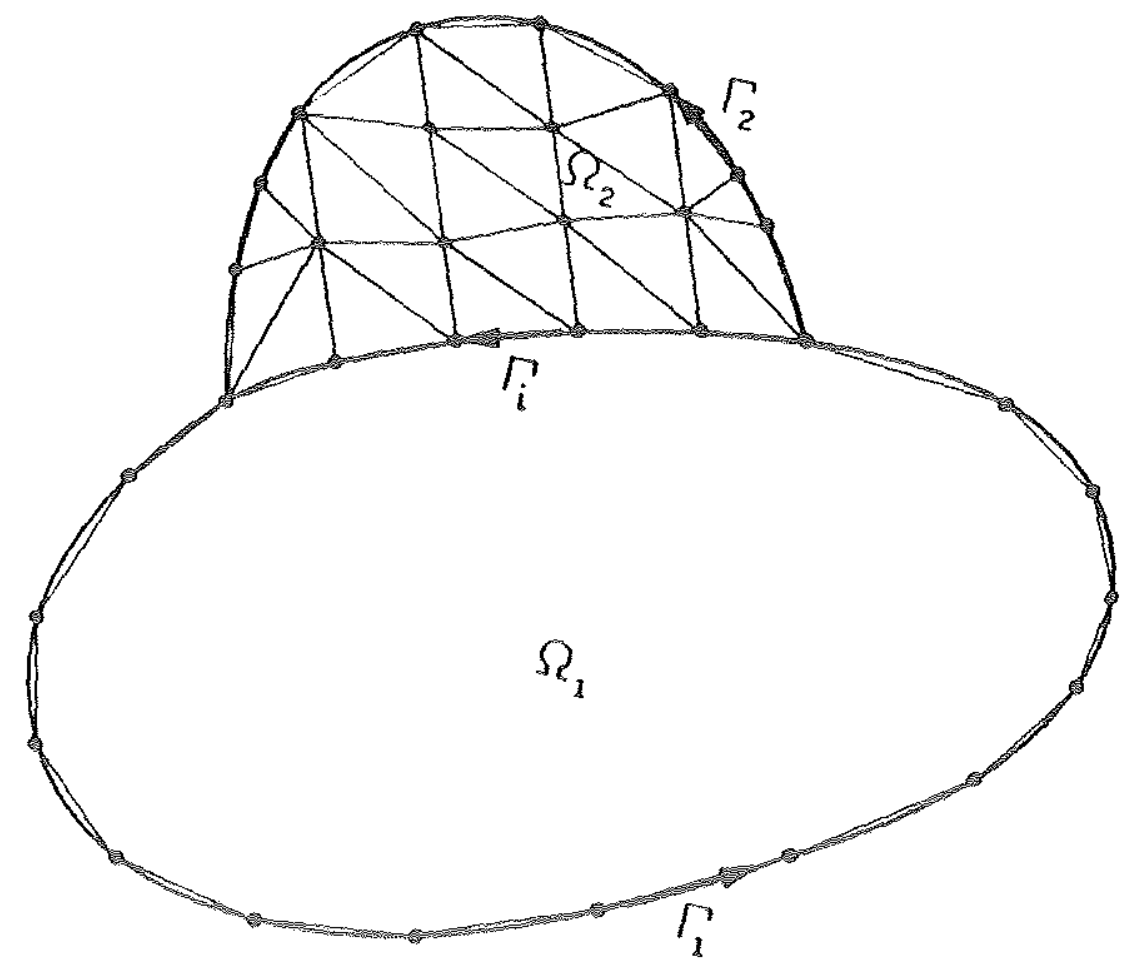

FIGURA 4.2 - Dominio discretizado em elementos finitos e elementos de contorno.

Duas alternativas são conhecidas para efetuar a combinação. Uma em que o sistema de equações obtido a partir do método dos elementos de contorno é transformado em um sistema similar, em forma, ao proveniente do método dos elementos finitos, e outra, pelo contrário, converte as equações referentes ao método dos elementos finitos, em um sistema com as características das equações do método dos elementos de contorno. 
Optou-se aqui, pela primeira alternativa, visto que um dos objetivos é o de se obter uma matriz de rigidez da fundação. Para tanto, vamos inicialmente reescrever a eq. (4.12), apresentando-a em termos de valores de componentes de forças nodais de interação:

$$
\mathbf{Q}=\mathbf{S}^{,-1} \mathbf{n}-\mathbf{Q}^{\mathbf{0}}
$$

sendo ,

$$
\mathbf{Q}^{0}=\mathbb{S}^{,-1} \mathbf{u}^{0}
$$

Lembrando que $\underset{\sim F}{\mathbb{F}}$ na eq. (4.16) representa forças nodais equivalentes, enquanto $Q$ na eq. (4.17) corresponde a valores de componentes de forças, portanto de diferentes naturezas, é necessário alterar-se a eq. (4.17) para se obterem sistemas de equações de formas análogas. Isso é feito multiplicando-se a eq. (4.17) pela matriz T que transforma componentes de forças nodais, em forças nodais equivalentes, podendose desta forma escrever:

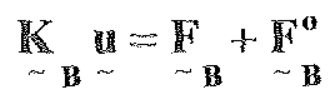

sendo,

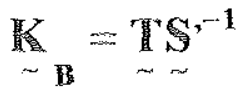

$$
\begin{aligned}
& { }_{\sim 8}^{T}=70
\end{aligned}
$$

e,

$$
\underset{\sim \mathrm{B}}{\mathrm{F}^{\mathbf{0}}}=\underset{\sim}{\mathrm{TQ}^{\mathbf{0}}}
$$

A eq. (4.19) é uma representação algébrica para domínios semi-infinitos eñ que as forças nodais (conhecidas e incógnitas) são aplicadas ao longo das linhas internas. Outras forças conhecidas atuando sobre o contorno ou sobre a superfície do meio contínuo são consideradas através de $\mathbb{Q}^{0}\left(\frac{\text { ou }{ }_{\sim}^{\circ}}{\mathbb{B}^{\circ}}\right)$

A matriz $\underset{\sim \text { B }}{\text { 笔 }}$ representa no sistema de coordenadas global uma matriz de rigidez equivalente, de ordem $3 \mathrm{~N}$, onde $\mathrm{N}$ é o número de nós das estacas. 
A associação do sólido semi-infinito e as estacas é agora efetuada combinandose adequadamente os sistemas expressos em (4.16) e (4.19), levando-se em consideração as condições de compatibilidade de deslocamentos e de equilíbrio, ao longo das interfaces estacas-meio-contínuo, isto é:

$$
\underset{\sim \mathbf{F}}{\mathbf{U}^{\mathbf{I}}}=\underset{\sim \mathbf{B}}{\mathbf{U}^{\mathbf{I}}}
$$

e,

$$
\underset{\sim F}{F^{I}}+\underset{\sim B}{\mathbf{F}^{I}}=\mathbf{0}
$$

em que, o indice $I$, indica a interface.

De uma forma geral, considerando-se as condições impostas nas eq. (4.23) e (4.24), para a associação dos sistemas apresentados em (4.16) e (4.19), obtém-se a seguinte estrutura para a representação do sistema

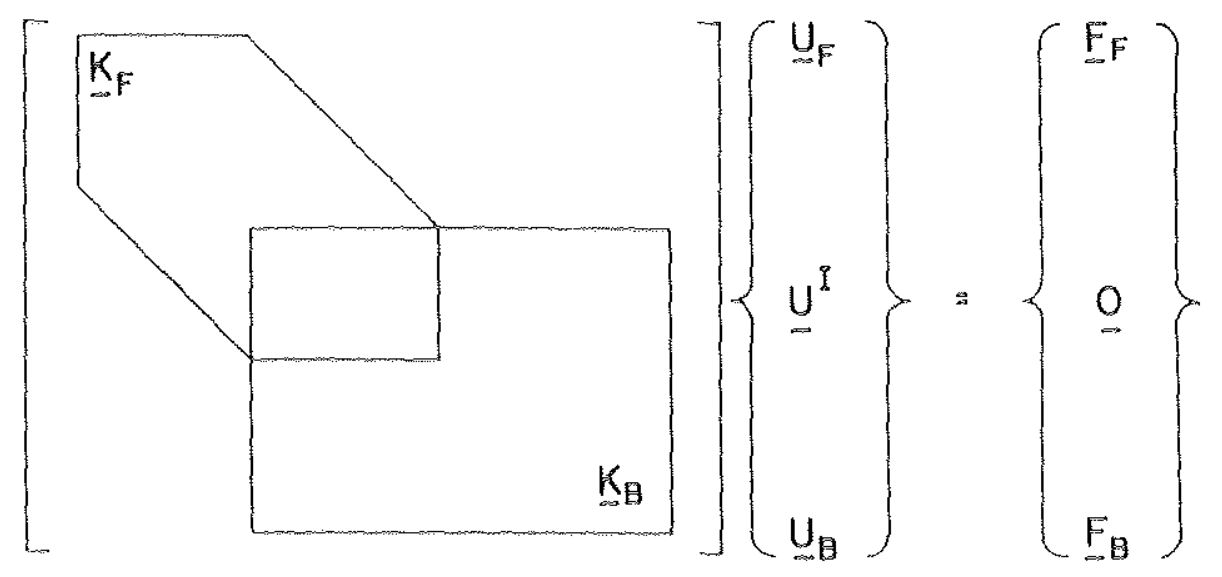

sendo, $\underset{\sim F}{\mathbb{K}}$ e $\underset{\sim}{K}$ as matrizes de rigidez obtidas a partir do método dos elementos finitos e de contorno, respectivamente, e $\mathrm{U}_{\sim}^{\mathrm{I}}$ o vetor de deslocamentos da interface.

O sistema representado na eq. (4.25) será reordenado, com o objetivo de agrupar os coeficientes de rigidez correspondentes aos pontos nodais nos topos das estacas, que passaremos a denominar de nós de superficie, e os demais pontos nodais das estacas, denominados nós internos. 
Assim, a matriz de rigidez pode ser apresentada como constituída por quatro submatrizes, e o sistema passa a ter a seguinte forma:

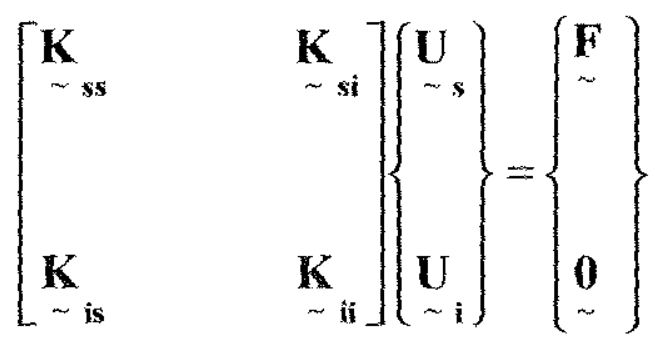

No próximo item, será apresentada a forma pela qual a partir da associação do MEC/MEF, se determina a matriz de rigidez da fundação.

\subsection{RIGIOEZ DO ELEMLNTO FUNDACAOO LM ESTACAS}

A partir da combinação dos dois métodos, o próximo passo é o de calcular, pela definição, a matriz de rigidez em relação a um ponto qualquer de cada um dos grupos de estacas sob cada um dos blocos de capeamento, podendo-se, para efeito de simplicidade, adotar-se o centro geométrico do grupo de estacas.

Isso se faz, impondo-se a cada um dos blocos, considerados perfeitamente rígidos, e com no minimo duas estacas, três deslocamentos unitários, segundo os eixos $\mathbb{X}_{1}$, $\mathbb{x}_{2}$ e $\mathbb{X}_{3}$, e três rotações unitárias, em tomo dos mesmos eixos, sempre em relação ao sistema global de coordenadas. Cada un desses seis graus de liberdade deve ser imposto de forma independente. A Fig. 4.3 mostra o deslocamento unitário em relação ao eixo $x_{1}$ e a rotação em tomo de $x_{2}$. Esses graus de liberdade são referidos aos pontos nodais localizados nos topos das estacas, aqui denominados como nós de superficie, deixando livres todos os demais nós, considerados internos. 

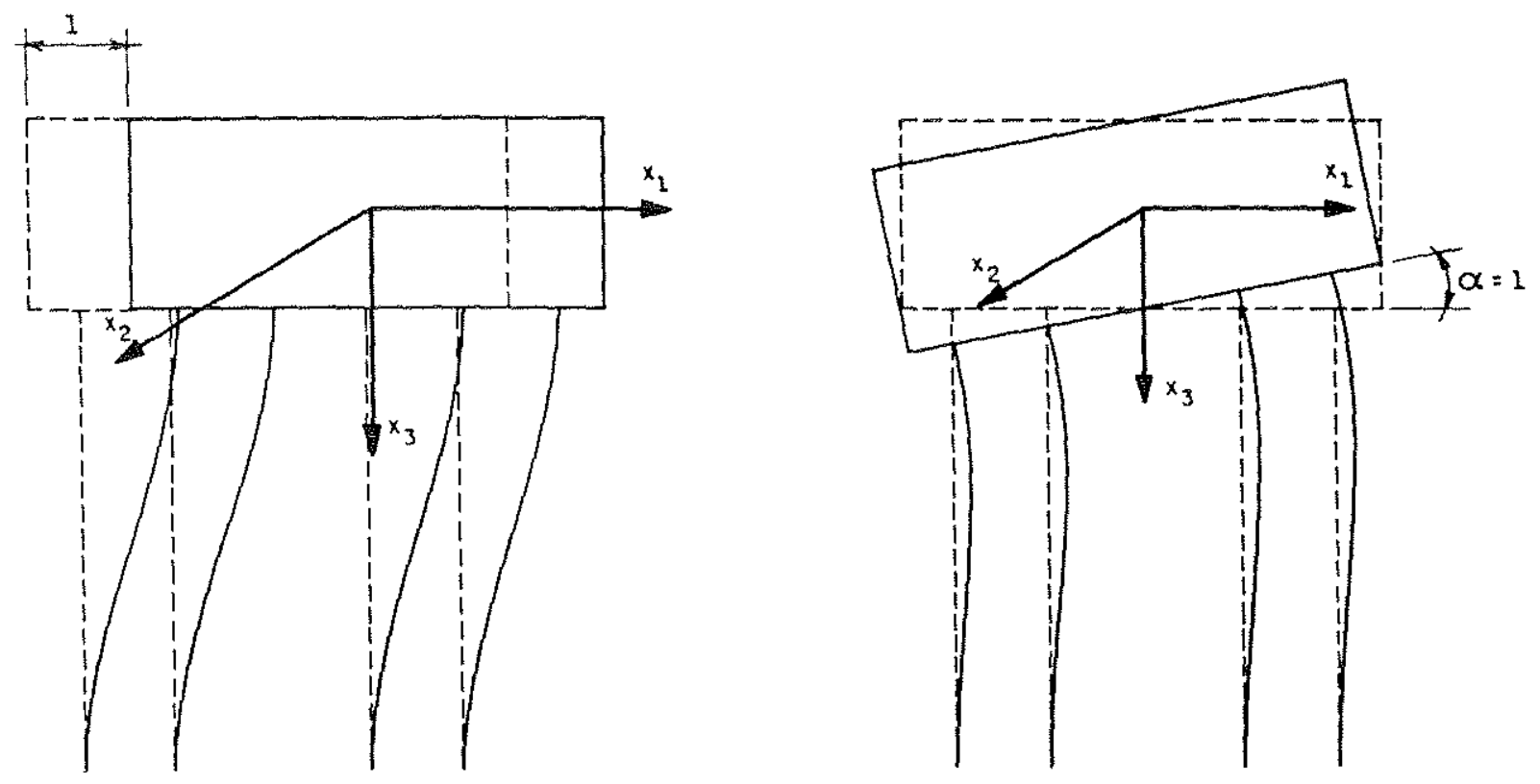

FIGURA 4.3 - Deslocamento unitário em relação ao eixo $\mathrm{x}_{1}$ e rotação em torno do eixo $x_{2}$.

As rotações em torno dos três eixos coordenados, são transformadas em deslocamentos, para cada um dos nós superiores das estacas do bloco, mantendo-se ainda tais rotações unitárias para cada um desses nós.

Assim, para exemplificar, tomemos um grupo de três estacas, ocupando os vértices de um triângulo, sob um bloco de capeamento rígido no qual são aplicados três deslocamentos unitários e três rotações também unitárias, que são transformadas em deslocamentos para os nós superiores. Tais deslocamentos e rotações produzirão seis vetores, que expressos em suas formas transpostas, assumem a forma:

$$
\begin{aligned}
& \underset{\sim 1}{\mathcal{U}^{\mathrm{T}}}=\left\{\begin{array}{llllllllllllllllll}
1 & 0 & 0 & 0 & 0 & 0 & 1 & 0 & 0 & 0 & 0 & 0 & 1 & 0 & 0 & 0 & 0 & 0
\end{array}\right\} \\
& \underset{-2}{\mathbb{U}^{\mathrm{T}}}=\left\{\begin{array}{llllllllllllllllll}
0 & 1 & 0 & 0 & 0 & 0 & 0 & 1 & 0 & 0 & 0 & 0 & 0 & 1 & 0 & 0 & 0 & 0
\end{array}\right\} \\
& \underset{-3}{U^{T}}=\left\{\begin{array}{llllllllllllllllll}
0 & 0 & 1 & 0 & 0 & 0 & 0 & 0 & 1 & 0 & 0 & 0 & 0 & 0 & 1 & 0 & 0 & 0
\end{array}\right\} \\
& \underset{\sim 4}{U^{T}}=\left\{\begin{array}{llllllllllllllllll}
0 & 0 & \mathbb{A}_{1} & 1 & 0 & 0 & 0 & 0 & \mathbb{A}_{2} & 1 & 0 & 0 & 0 & 0 & \mathbb{A}_{3} & 1 & 0 & 0
\end{array}\right\} \\
& \underset{\sim 5}{U^{\mathrm{T}}}=\left\{\begin{array}{llllllllllllllllll}
0 & 0 & \mathbb{B}_{1} & 0 & 1 & 0 & 0 & 0 & \mathbb{B}_{2} & 0 & 1 & 0 & 0 & 0 & B_{3} & 0 & 1 & 0
\end{array}\right\}
\end{aligned}
$$




$$
\underset{\sim 6}{U^{\mathrm{T}}}=\left\{\begin{array}{llllllllllllllllll}
\mathrm{C}_{1} & \mathrm{D}_{1} & 0 & 0 & 0 & 0 & \mathrm{C}_{2} & \mathrm{D}_{2} & 0 & 0 & 0 & 0 & C_{3} & \mathrm{D}_{3} & 0 & 0 & 0 & 0
\end{array}\right\}
$$

sendo que, $\underset{\sim}{\mathbf{U}}$ é o vetor correspondente ao i-ésimo grau de liberdade para um determinado bloco de estacas.

Os parâmetros A , B , C e D são distâncias dadas por:

$$
\begin{aligned}
& A_{i}=x_{2}(\text { no } i)-x_{2} \text { (c.geom.) } \\
& B_{i}=x_{1} \text { (c.geom.) }-x_{1}(\text { no } i) \\
& C_{i}=x_{2} \text { (c.geom.) }-x_{2}(\text { no } i) \\
& D_{i}=x_{1} \text { (noi) }-x_{1} \text { (c.geom.) }
\end{aligned}
$$

O conjunto formado pelos deslocamentos obtidos para os nós superiores das estacas do bloco considerado e mais cinco valores nulos para cada um dos nós superiores das estacas dos outros blocos, constitui um vetor que deve ser levado ao sistema apresentado na eq. (4.26). Quando todos os movimentos unitários tiverem sido considerados, ter-se-á um total de $6 \mathrm{NB}$ vetores, onde NB é o número de blocos, sendo o sistema final representado por:

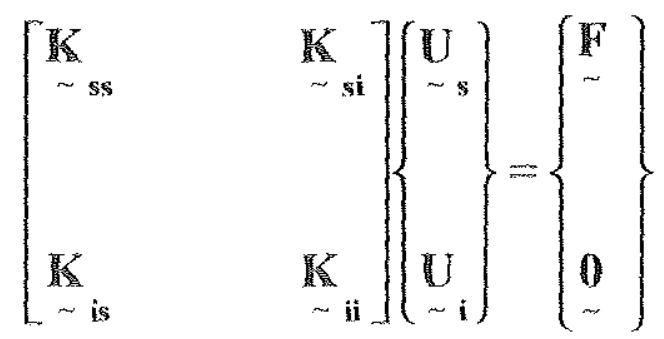

sendo que, as submatrizes $\underset{\sim \mathrm{ss}}{\mathrm{K}}(5 \mathrm{Nex} 5 \mathrm{NE}), \underset{\sim \mathrm{si}}{\mathrm{K}}[5 \mathrm{Nex} 5(\mathrm{NN}-\mathrm{NE})], \underset{\sim \text { is }}{\mathrm{K}}[5(\mathrm{NN}-$ NE) $\times 5 N E]$ e $\underset{\sim \text { ii }}{{ }_{\text {i }}}[5(\mathrm{NN}-\mathrm{NE}) \times 5(\mathrm{NN}-\mathrm{NE})]$ são compostas por coeficientes de $\underset{\sim}{\sim} \mathrm{K}$ $\underset{\sim \mathrm{K}}{K}, \underset{\sim \mathrm{s}}{U}(5 \mathrm{NEx} 6 \mathrm{NB})$ é a matriz que contém as componentes de deslocamentos e rotações dos nós da superficie, enquanto $\underset{\sim i}{U}[5(\mathrm{NN}-\mathrm{NE}) \times 6 \mathrm{NB}]$ contém os deslocamentos e rotações dos nós intemos das estacas, e T $^{\text {T }}$ (5NEx6NB) é a matriz que contém as forças nodais equivalentes incógnitas que tratada de forma conveniente, dará origem à matriz de rigidez da fundação. 
$\mathrm{Na}$ representação das matrizes feitas acima, $\mathrm{NN}$ é o número total de nós das estacas, NE o número total de estacas e NB é o número de blocos de capeamento (igual ao número de nós da superestrutura ligados à fundação).

O sistema expresso na eq. (4.29), pode ser reescrito de outra forma, sem no entanto alterá-lo, ficando:

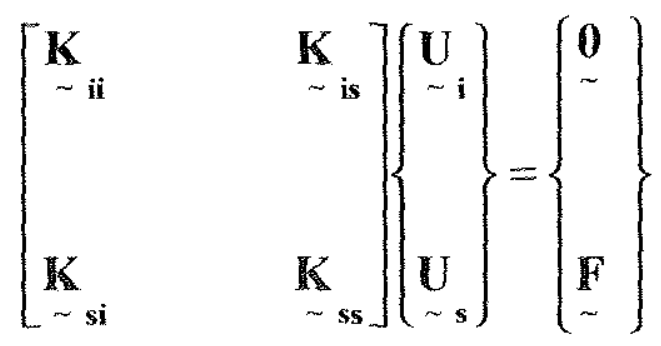

Dois sistemas de equações podem ser obtidos a partir da eq. (4.30), escritos como:

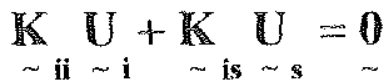

$$
\begin{aligned}
& K_{\sim s i} U+I_{\sim s s} U=T
\end{aligned}
$$

A partir da eq. (4.31), pode-se determinar $\underset{\sim i}{U}$, como:

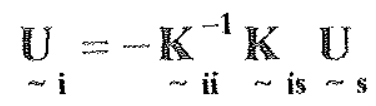

Substituindo-se a eq. (4.33) na eq. (4.32), obtém-se:

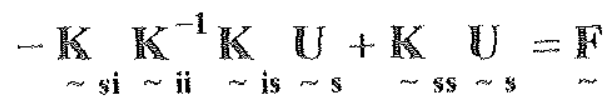

A eq. (4.34), é reescrita colocando-se o vetor $\underset{\sim s}{U}$, em evidência, ficando:

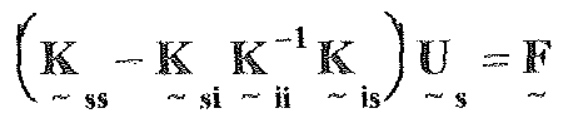

ou,

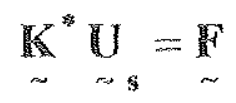

em que:

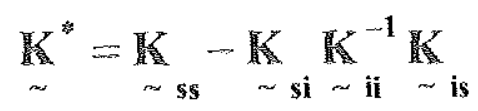


Um procedimento mais elegante, é o de se empregar a técnica de condensação estática, apresentada por MEEK (1971) e WILSON (1974), e que consiste em transformar a submatriz $\underset{\sim}{\mathbf{K}}$ em uma matriz identidade e $\underset{\sim \text { si }}{\mathbf{K}}$ em uma matriz nula, passando o sistema (4.30), a ser expressa como:

$$
\left[\begin{array}{cc}
\mathbf{I} & \mathbf{K}^{*} \\
\sim & \sim \mathrm{is} \\
\mathbf{0} & \mathbf{K}_{\sim \mathrm{ss}}^{*}
\end{array}\right]\left\{\begin{array}{c}
\mathbf{U} \\
\sim \mathrm{i}
\end{array}\right\}=\left\{\begin{array}{c}
\mathbf{0} \\
\sim \\
\mathrm{F}
\end{array}\right\}
$$

O segundo sistema de equações de (4.38), pode ser representado como:

$$
\underset{\sim \text { ss }}{U \sim \mathrm{s}}=\underset{\sim}{U}
$$

ou,

$$
K^{*} \mathbb{U}=\mathbb{P}
$$

Utilizando-se a técnica de condensação estática, desenvolveu-se um algoritmo, que foi implementado em um programa de computador, corn a finalidade de se calcular a matriz de rigidez da fundação em estacas $\widetilde{K}$, de ordem $6 \mathrm{NB}$.

Essa matriz é obtida a partir da matriz $T$, obedecendo-se as seguintes etapas:

Os coeficientes da matriz $\widetilde{\Gamma}$, são obtidos a partir da matriz $\overline{\mathrm{T}}$, somando-se as contribuições dos coeficientes de cada uma das estacas do bloco, relativas à respectiva direção.

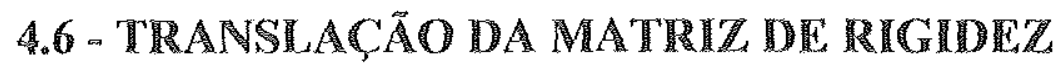

A matriz de rigidez $\bar{K}$ determinada em relação a cada um dos centros geométricos dos grupos de estacas, deverá ser transferida para os correspondentes 
pontos de ligação com a superestrutura, através de transformações convenientes, de acordo com MEEK (1971).

Considerem-se as forças $\overline{\mathbf{F}}_{1}, \overline{\mathbf{F}}_{2}$ e $\overline{\mathbf{F}}_{3}$ e os momentos $\overline{\mathbf{M}}_{1}, \overline{\mathbf{M}}_{2}$ e $\overline{\mathbf{M}}_{3}$ aplicados no ponto $\overline{\mathbf{O}}$, como mostra a Figura 4.4. Para a transferência dessas forças e momentos para o ponto $\boldsymbol{O}$, pode-se escrever:

$$
\left\{\begin{array}{c}
F_{1} \\
F_{2} \\
F_{3} \\
M_{1} \\
M_{2} \\
M_{3}
\end{array}\right\}=\left[\begin{array}{cccccc}
1 & 0 & 0 & 0 & 0 & 0 \\
0 & 1 & 0 & 0 & 0 & 0 \\
0 & 0 & 1 & 0 & 0 & 0 \\
0 & -e_{3} & e_{2} & 1 & 0 & 0 \\
e_{3} & 0 & -e_{1} & 0 & 1 & 0 \\
-e_{2} & e_{2} & 0 & 0 & 0 & 1
\end{array}\right]\left\{\begin{array}{c}
F_{1} \\
\bar{F}_{2} \\
\bar{F}_{3} \\
\bar{M}_{1} \\
\bar{M}_{2} \\
\bar{M}_{3}
\end{array}\right\}
$$

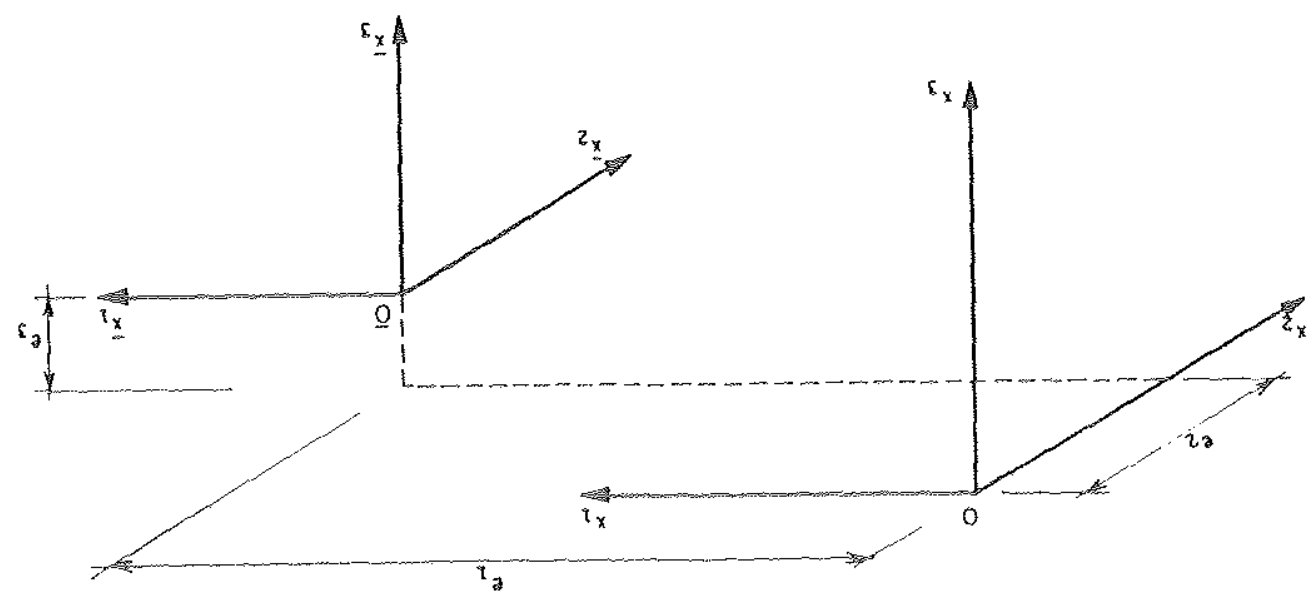

FIGURA 4.4 - Translação da rigidez do ponto $\overline{\mathbf{O}}$ para o ponto $\mathbf{O}$.

Resumidamente, tem-se:

$$
\mathrm{T}=\mathrm{L} \overline{\mathrm{T}}
$$

onde $\overline{\mathbb{F}}$ e $\bar{T}$ são as forças e momentos atuantes em $\overline{\mathrm{O}}$ e $\mathrm{O}$, respectivamente, e $\mathrm{L}$ é a matriz que realiza a transformação. 
A matriz $\mathbf{L}$, pode ser particionada em quatro submatrizes, e apresentada como:

$$
\underset{\sim}{\mathbf{L}}=\left[\begin{array}{cc}
\mathbf{I} & \mathbf{0} \\
\tilde{\mathbf{W}} & \tilde{\mathbf{I}} \\
\sim & \sim
\end{array}\right]
$$

A sua matriz inversa, pode ser escrita como:

$$
\underset{\sim}{\mathbf{L}^{-1}}=\left[\begin{array}{cc}
\mathbf{I} & 0 \\
\sim & \sim \\
\mathbf{W}^{\mathbf{T}} & \mathbf{I}
\end{array}\right]
$$

E a transformação inversa, é dada por:

$$
\bar{F}=L^{-1} \mathrm{~T}
$$

A transformação correspondente aos deslocamentos e rotações, que ocorre do ponto $\mathrm{O}$ para o ponto $\overline{\mathrm{O}}$, é dada por:

$$
\widetilde{U}=\mathbb{L}^{\mathrm{T}} \mathrm{U}
$$

onde, $\widetilde{U}$ e $\quad$ Uão os deslocamentos e rotações relativos aos ponto $\overline{\mathrm{O}}$ e respectivamente, e $L^{\mathrm{T}}$ é a transposta da matriz $\mathrm{L}$.

Para o ponto $\overline{\mathrm{O}}$, sendo $\overline{\mathrm{K}}$ a matriz de rigidez da fundação que relaciona forças e deslocamentos, pode-se escrever:

$$
\overline{\mathrm{K} U}=\overline{\mathrm{T}}
$$

Substituindo-se na eq. (4.46) as equações (4.44) e (4.45), obtém-se:

$$
\underset{\sim}{\operatorname{K} L^{T}} \underset{\sim}{U}=L_{\sim}^{-1} T
$$

Multiplicando-se a expressão de (4.47) pela matriz L tem-se:

$$
\underset{\sim}{\mathbb{K}} \mathrm{L}_{\sim}^{\mathrm{T}} \mathrm{U}=\underset{\sim}{\mathrm{T}}
$$

ou,

$$
K^{K} U=\mathbb{T}
$$


sendo,

$$
\underset{\sim}{\mathbf{K}}=\underset{\sim \sim \sim}{\mathbf{L}} \mathbf{L}_{\sim}^{\mathbf{T}}
$$

sendo, $K^{\prime}$ a matriz de rigidez da fundação, transladada para os pontos de ligação bloco-superestrutura e $\overline{\mathbf{K}}$ a matriz de rigidez da fundação referida aos centros geométricos dos respectivos grupos de estacas.

É conveniente lembrar que a matriz de rigidez $\overline{\mathbf{K}}$ é composta de (NB $\times$ NB) submatrizes, onde NB é o número de blocos de estacas, existentes na fundação, podendo ser representada por:

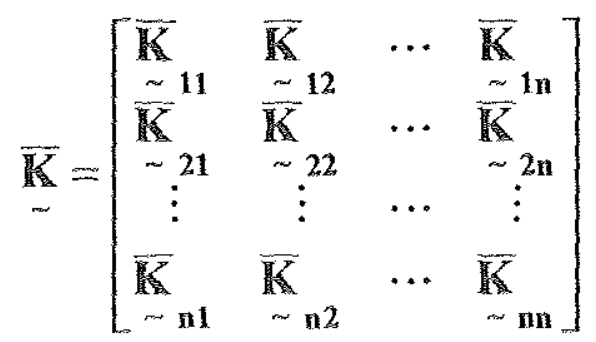

sendo que, $\underset{\sim \mathrm{K}}{\overline{\mathrm{K}}}$ é a submatriz de rigidez de ordem seis, que relaciona forças e deslocamentos dos blocos "i" e "j".

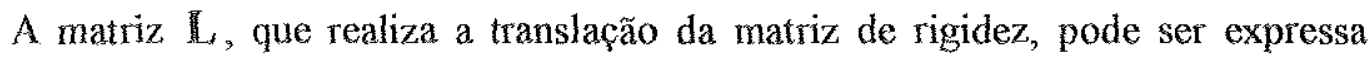
como:

$$
\underset{\sim}{L}=\left[\begin{array}{cccc}
L & 0 & \cdots & 0 \\
\sim 1 & \sim & & \tilde{0} \\
0 & L & \cdots & 0 \\
\tilde{0} & \sim 2 & \cdots & \tilde{0} \\
\tilde{0} & \tilde{0} & \cdots & \sim \\
\sim & \sim & & \sim
\end{array}\right]
$$

onde $\mathbb{L}$, é a matriz da expressão (4.40), que translada a matriz de rigidez do centro geométrico das estacas do bloco i, para o correspondente ponto de ligação blocosuperestrutura.

A matriz de rigidez final da fundação relacionando deslocamentos e forças nodais é assimétrica devido a natureza das equações integrais usadas durante seu desenvolvimento. No entanto, esta matriz assimétrica deverá ser combinada a uma 
matriz simétrica oriunda da formulação de elementos finitos aplicada à superestrutura do edifício, é então, resolvida considerando as características de cada uma das subestruturas envolvidas.

Uma alternativa, que parece ser a mais recomendável, consiste em proceder uma subestruturação no sistema algébrico resultante da aplicação do método dos elementos finitos na superestrutura, de forma que o sistema de equações final seja condensado para os mesmos pontos de ligação pilar-bloco sobre estacas.

Resolve-se o sistema final de equações após a combinação dessas matrizes, obtendo-se os deslocamentos dos nós de ligação. Conhecidos os deslocamentos e as correspondentes forças nodais, pode-se determinar os deslocamentos para todos os nós, tanto da superestrutura quanto para os conjuntos solo-estacas, assim como os esforços de extremidades para os elementos das duas subestruturas.

\subsection{LINHAS DE CARGA NO DOMINIO}

A eq. (4.9), permite calcular os deslocamentos associados aos pontos tomados ao longo das linhas internas. A influência das linhas de carga em seu próprio deslocamento, é representada pelo último termo da eq.(4.9).

A aproximação em linha, como considerado no item 4.2 , introduz singularidade

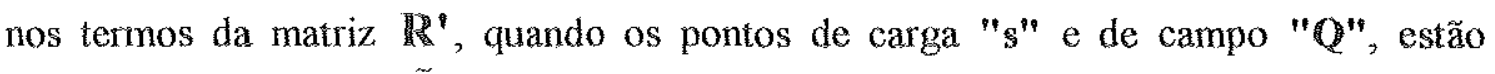
localizados no mesmo elemento. Por esta razão, o uso do conceito de linha de carga para simular a ação de uma estaca cravada no meio semi-infinito, deve ser modificado, sendo portanto necessário considerar o diâmetro da estaca, e em conseqüência as integrações deverão ser efetuadas ao longo da superficie de interação entre a estaca e o meio contínuo semi-infinito.

Estas integrações são efetuadas numericamente, entre o ponto de carga "s", localizado no eixo da estaca e o ponto de campo "Q", na superficie da estaca. Para determinar as coordenadas do ponto campo em relação ao ponto fonte, admite-se uma estaca em posição qualquer no meio contínuo. Seu eixo central, em relação a um sistema de coordenadas global, determina os ângulos $\alpha, \beta$ e $\delta$, respectivamente em 
relação aos eixos $\mathbf{x}_{1}, \mathbf{x}_{2}$ e $\mathbf{x}_{\mathbf{3}}$. A Fig. 4.5 mostra uma seção transversal da estaca, de raio $\mathbf{r}_{\text {f. }}$ As coordenadas de um ponto $\mathbf{b}$ na superfície da estaca, podem ser escritas em função das coordenadas de um ponto $\mathbf{k}$ no centro desta seção, pelo qual fica definido um sistema de coordenadas locais, como:

$$
\begin{aligned}
& \overline{\mathbf{x}}_{1}^{\mathbf{b}}=\overline{\mathbf{x}}_{1}^{\mathbf{k}} \\
& \overline{\mathbf{x}}_{2}^{\mathbf{b}}=\overline{\mathbf{x}}_{2}^{\mathbf{k}}+\mathbf{r}_{\mathrm{f}} \cos \theta \\
& \overline{\mathbf{x}}_{3}^{\mathbf{b}}=\overline{\mathbf{x}}_{3}^{\mathbf{k}}+\mathbf{r}_{\mathrm{f}} \operatorname{sen} \theta
\end{aligned}
$$

As eq. (4.53), representadas na forma matricial, fica:

$$
\underset{\sim}{\mathbf{x}}=\underset{\sim}{\mathbf{x}}+\underset{\sim}{\mathbf{M}} \mathbf{r}_{\mathbf{f}}
$$

onde o vetor $\mathbf{M}$, é dada por:

$$
\mathbb{M}=\left\{\begin{array}{c}
0 \\
\cos \theta \\
\operatorname{sen} \theta
\end{array}\right\}
$$

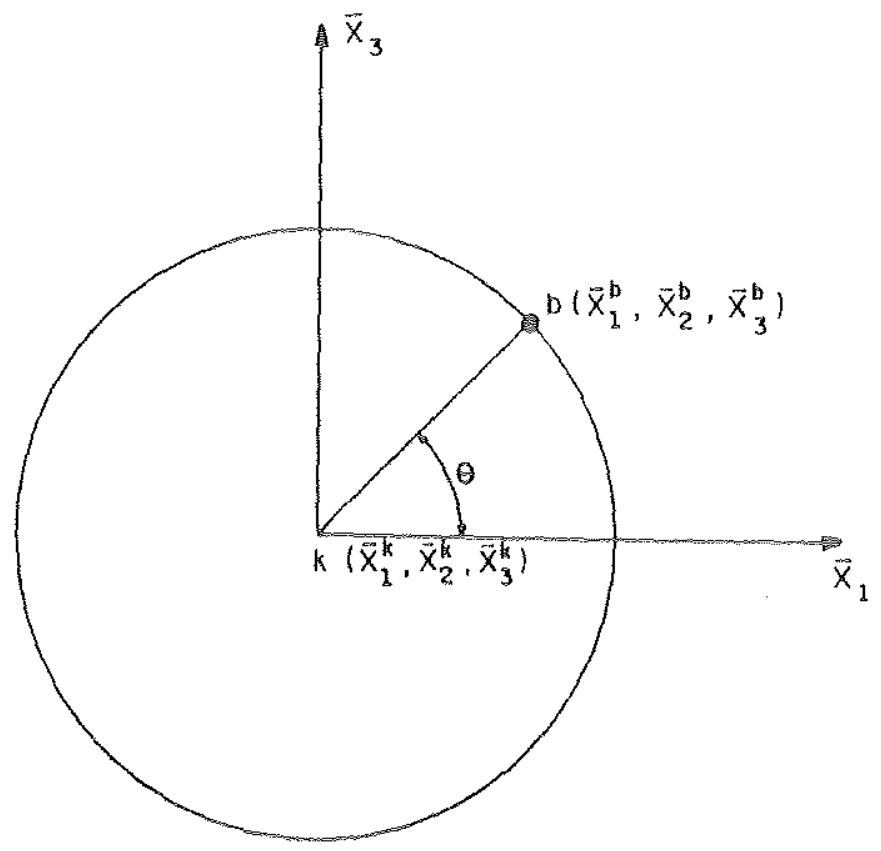

FIGURA 4.5 - Sistema de coordenadas locais para uma seção da estaca.

As coordenadas do ponto $\mathbf{b}$, no sistema global de coordenadas podem ser expressas, como: 


$$
\mathbf{x}^{b}=\mathbf{R}^{* \mathbf{T}} \overline{\mathbf{x}}^{\mathbf{b}}
$$

sendo, $\mathbf{x}_{\sim}^{b}$ as coordenadas do ponto $\mathbf{b}$ no sistema global de coordenadas $\mathrm{e} \underset{\sim}{\mathbf{R}}$, a matriz transposta que relaciona os sistemas de coordenas local e o global. A matriz $\mathbf{R}^{* \mathbf{T}}$, é dada por:

$$
\underset{\sim}{* T}=\left[\begin{array}{ccc}
\cos \alpha & \frac{\cos \beta}{\sqrt{\cos ^{2} \alpha+\cos ^{2} \beta}} & -\frac{\cos \alpha \cos \delta}{\sqrt{\cos ^{2} \alpha+\cos ^{2} \beta}} \\
\cos \beta & \frac{\cos \alpha}{\sqrt{\cos ^{2} \alpha+\cos ^{2} \beta}} & -\frac{\cos \beta \cos \delta}{\sqrt{\cos ^{2} \alpha+\cos ^{2} \beta}} \\
\cos \delta & 0 & \sqrt{\cos ^{2} \alpha+\cos ^{2} \beta}
\end{array}\right]
$$

e, para o caso particular de estaca na posição vertical, a matriz $\mathbb{R}^{*} \mathbf{T}$, toma a forma:

$$
\underset{\sim}{R^{* \mathrm{~T}}}=\left[\begin{array}{ccc}
0 & 0 & -1 \\
0 & 1 & 0 \\
1 & 0 & 0
\end{array}\right]
$$

Substituindo-se, o vetor de coordenadas locais do ponto b da eq. (4.54) na eq. (4.56), tem-se:

$$
x^{b}=R_{\sim}^{* T}\left(x^{k}+M r_{f}\right)
$$

As coordenadas no sistema local do ponto $k$, na eq. (4.59), escritas no sistema global, ficam:

$$
x^{b}=\mathbb{R}_{\sim}^{m}\left(\mathbb{R}_{\sim}^{k} x^{k}+\underset{\sim}{M} R_{f}\right)
$$

o que resulta, em:

$$
x^{b}=x^{k}+\underset{\sim}{N} p_{i}
$$

onde, o vetor $\mathbb{N}$, vale: 


$$
\underset{\sim}{\sim}=\frac{1}{\sqrt{\cos ^{2} \alpha+\cos ^{2} \beta}}\left\{\begin{array}{c}
-\cos \beta \cos \theta-\cos \alpha \cos \delta \operatorname{sen} \theta \\
\cos \alpha \cos \theta-\cos \beta \cos \delta \operatorname{sen} \theta \\
\left(\cos ^{2} \alpha+\cos ^{2} \beta\right) \operatorname{sen} \theta
\end{array}\right\}
$$

Assim, qualquer ponto "campo" na superficie de uma estaca cilíndrica, tem sua posição definida em relação a um ponto "fonte" localizado no eixo da estaca.

Estas integrais que correspondem aos coeficientes da matriz $\mathbf{R}^{\prime}$, foram alvo de uma cuidadosa análise, tendo como objetivo o aumento de precisão. Para tanto, optou-se por efetua-las de maneira diversa, conforme a posição dos pontos "s" e "Q" nas estacas.

Em todos os casos, as integrações ao longo das estacas são duplas e calculadas numericamente, sendo que, uma é no sentido circunferencial de 0 a 2 pi, e a outra ao longo do eixo da estaca.

No entanto, quando os pontos de carga "s" e de campo "Q", estão a uma distância menor ou igual a metade do comprimento do elemento, que está sendo integrado, mesmo que em estacas distintas, é utilizada a técnica de integração por subelementos.

Observe-se que, para distâncias maiores que 2,5 vezes o comprimento do elemento que está sendo integrado, o erro entre os valores obtidos por integração dupla ou simples (considerando a estaca objeto da integração como uma linha) é da ordem de $3.10^{-7}$. Este valor é muito pequeno, podendo-se, portanto, optar por qualquer uma das duas formas de integração.

As forças de interação ao longo das barras, são aproximadas por funções polinomiais e representadas como componentes de forças nodais. Assim, um modelo foi desenvolvido, onde o elemento adotado é o linear, e a função interpoladora para as forças na superfĩcie de contato é a quadrática. A Figura 4.6 , mostra o elemento, os pontos nodais e as funções interpoladoras. 

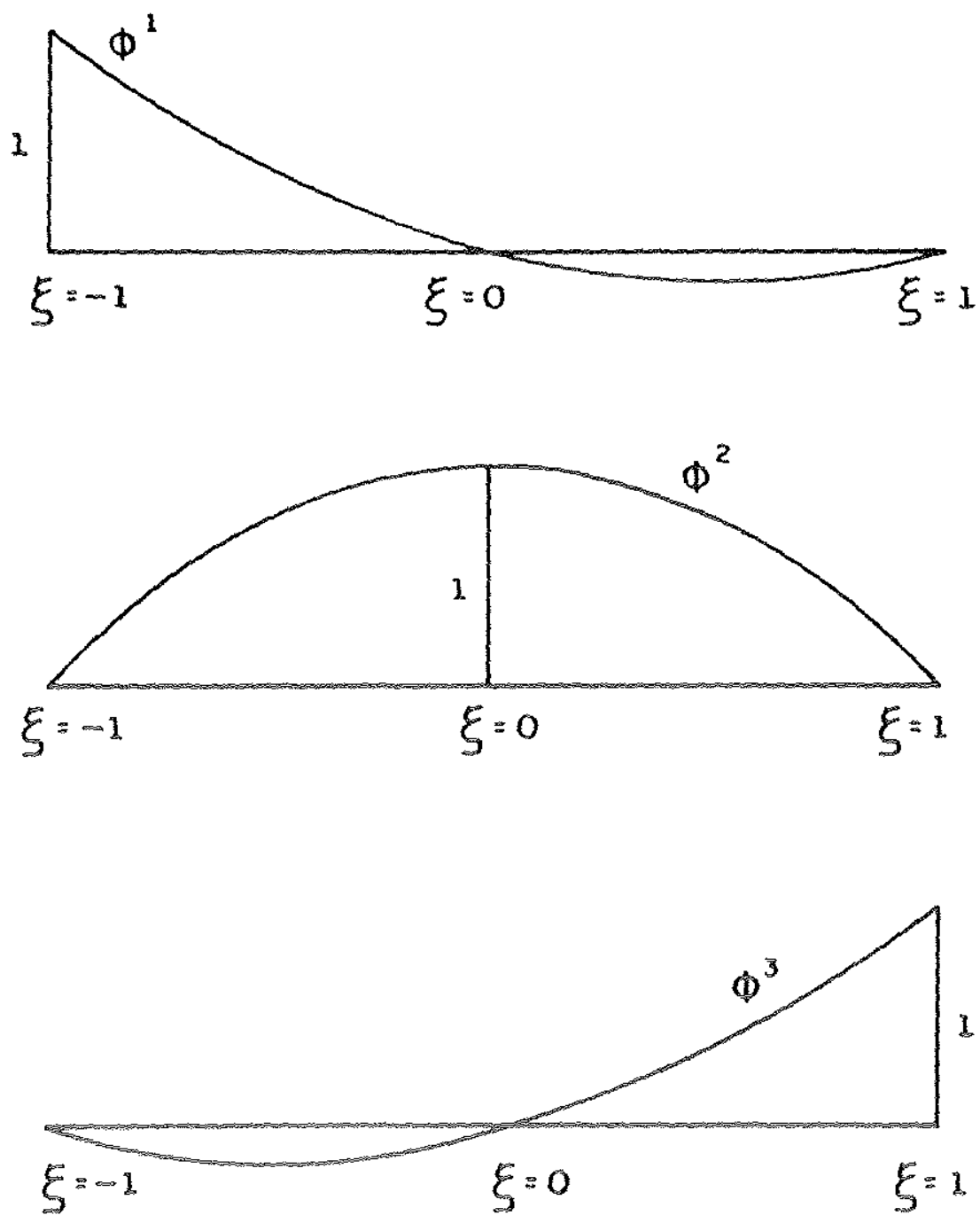

FIGURA 4.6 - Elemento linear contínuo, e as respectivas funções de interpolação.

$\stackrel{\phi}{\sim}^{\mathrm{T}}=\left[\begin{array}{ccccccccc}\phi^{1} & 0 & 0 & \phi^{2} & 0 & 0 & \phi^{3} & 0 & 0 \\ 0 & \phi^{1} & 0 & 0 & \phi^{2} & 0 & 0 & \phi^{3} & 0 \\ 0 & 0 & \phi^{1} & 0 & 0 & \phi^{2} & 0 & 0 & \phi^{3}\end{array}\right]$

Dessa forma, as variáveis do contorno podem ser expressas como nas eq. (4.5):

$\underline{\sim}=\phi^{\mathrm{T}} \underline{\sim}^{\mathrm{n}}$

$q=\phi^{\mathrm{T}} \mathrm{Q}^{\mathrm{n}}$ 
sendo:

$$
\begin{aligned}
\underset{\sim}{u}=\left\{\begin{array}{l}
\mathbf{u}_{1} \\
\mathbf{u}_{2} \\
\mathbf{u}_{3}
\end{array}\right\} & \underset{\sim}{\mathbf{q}}=\left\{\begin{array}{l}
\mathbf{q}_{1} \\
\mathbf{q}_{2} \\
\mathbf{q}_{3}
\end{array}\right\} \\
\mathbf{U}^{\mathbf{n}}=\left\{\begin{array}{l}
\mathbf{U}_{1}^{1} \\
\mathbf{U}_{2}^{1} \\
\mathbf{U}_{3}^{1} \\
\mathbf{U}_{1}^{2} \\
\mathbf{U}_{2}^{2} \\
\mathbf{U}_{3}^{2} \\
U_{1}^{3} \\
U_{2}^{3} \\
U_{3}^{3}
\end{array}\right\} & \mathbb{Q}^{\mathbf{n}}=\left\{\begin{array}{l}
\mathbf{Q}_{1}^{1} \\
\mathbf{Q}_{2}^{1} \\
Q_{3}^{1} \\
Q_{1}^{2} \\
Q_{2}^{2} \\
Q_{3}^{2} \\
Q_{1}^{3} \\
Q_{2}^{3} \\
Q_{3}^{3}
\end{array}\right\}
\end{aligned}
$$

onde, $U_{j}^{i}$ e $Q_{j}^{i}$ são a componente de deslocamento e de forças de superficie na interface solo-estaca, no nó i e direção $\mathbf{j}$, respectivamente. Essas forças de superfície são admitidas constantes no contorno de uma dada seção transversal da estaca e variável ao longo de seu comprimento. Tais componentes de forças de superficie devem ser transformadas em componentes de forças de interação, variável ao longo do comprimento da estaca, da seguinte forma:

$$
\mathrm{P}_{\mathbf{j}}^{\mathrm{i}}=2 \pi \mathrm{r}_{\mathbf{f}} \mathrm{Q}_{\mathbf{j}}^{\mathrm{i}}
$$

sendo que:

$\mathbf{r}_{\mathrm{f}}$ é o raio do fuste da estaca no elemento em análise.

À Fig. 4.7 mostra-se, em representação esquemática, a distribuição das componentes de forças de interação para um elemento de estaca.

Para completar a combinação do método dos elementos de contorno com o método dos elementos finitos, as componentes de forças de interação devem ser alteradas para forças nodais equivalentes. Esta mudança é feita a partir da energia potencial devido ao carregamento, que é dada por: 

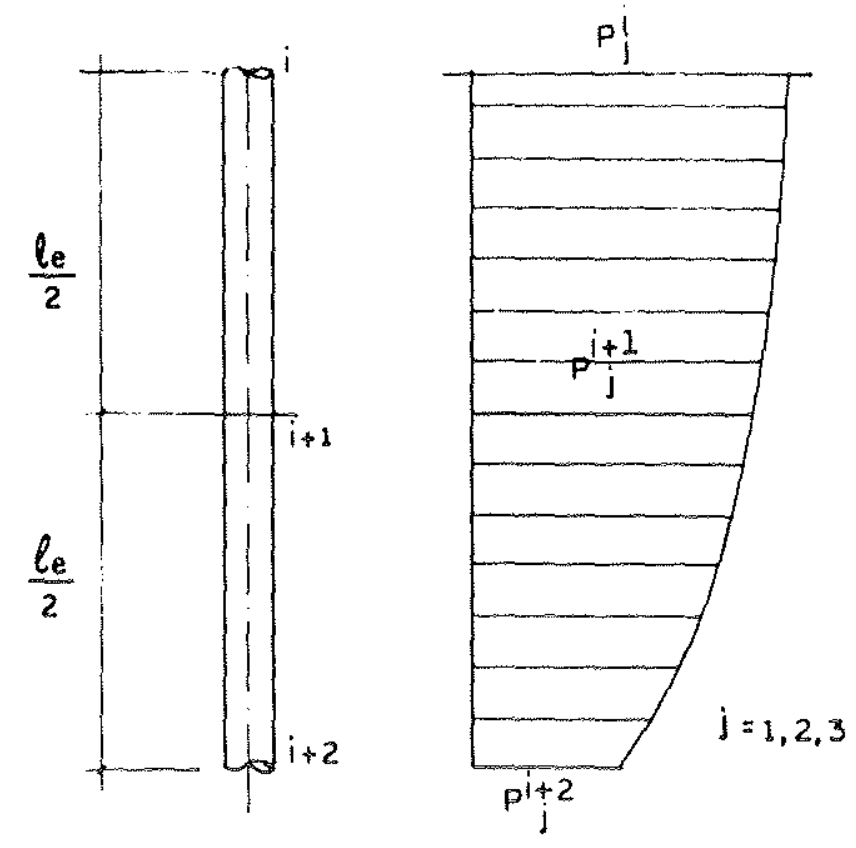

FIGURA 4.7 - Representação da distribuição das forças de interação em um elemento de estaca.

$$
\Omega=-2 \pi \mathrm{r}_{\mathrm{f}} \int_{0}^{\ell} \mathrm{T} \mathrm{p} \ell
$$

sendo $\ell$, o comprimento do elemento.

Escrevendo-se a expressão da energia potencial das cargas externas em termos de coordenadas adimensionais, tem-se:

$$
\Omega=-2 \pi r_{\mathrm{r}} \frac{\ell}{2} \int_{-1}^{1} \mathrm{~d} \mathrm{U}^{\mathrm{T}} \mathrm{d} \xi
$$

As variáveis, no caso, deslocamentos u e forças distribuidas ao longo do elemento, podem ser expressas em termos de componentes nodais, que substituídas na eq. (4.68), resulta:

$$
\Omega=-2 \pi r_{i} \frac{\ell}{2} U^{n, T} \int_{-1}^{1} \chi^{T} \omega d \xi P^{n}
$$


onde, $\chi$ e $\Phi$ são as funções aproximadoras para deslocamentos e forças distribuídas, definidas no método dos elementos finitos.

Calculando-se a variação da energia potencial do carregamento, tem-se:

$$
\delta \Omega=-2 \pi \mathrm{r}_{\mathrm{f}} \frac{\ell}{2} \delta U_{\sim}^{\mathrm{n}, \mathrm{T}} \int_{-1}^{1} \chi_{\sim}^{\mathrm{T}} \underset{\sim}{\Phi} \xi_{\mathcal{\sim}} \mathbf{P}^{\mathrm{n}}
$$

A partir da variação da energia potencial, dada na equação acima, as forças nodais equivalentes são calculadas como:

$$
\underset{\sim \mathrm{B}}{\mathbf{F}}=2 \pi r_{\mathrm{f}} \frac{\ell}{2} \int_{-1}^{1} \chi_{\sim}^{\mathrm{T}} \underset{\sim}{\boldsymbol{\Phi}} \underset{\sim}{\mathbf{P}^{\mathrm{n}}}
$$

As forças nodais equivalente, $\underset{\sim \mathbf{B}}{\mathbf{F}}$, de acordo com a eq. (4.71), são calculadas em função das componentes de forças de interação $\mathbf{P}^{n}$. Assim, pode-se escrever que:

$$
\underset{\sim B}{\mathbb{F}}=2 \pi \mathrm{r}_{\mathrm{f}} \operatorname{TP}_{\sim \sim}
$$

A eq. (4.72) tem a mesma representação matricial que a eq. (4.21). Portanto, a matriz $\underset{\sim}{T}$, pode ser escrita para $\mathbb{N}_{e}$ elementos de barras, como:

$$
\mathrm{T}=\frac{\ell}{2} \sum_{\mathbf{j}=1}^{\mathrm{N}_{\mathrm{e}}} \int_{-1}^{1} \chi^{\mathrm{T}} \Phi \mathrm{d} \xi
$$

onde $\ell_{\mathbf{j}}$ é o comprimento do elemento $\mathbf{j}$.

A função aproximadora das forças de interação, $\mathbf{\Phi}$, é adotada do $2^{\circ}$ grau, enquanto que, a função aproximadora dos deslocamentos, $\chi$, para as direções $\mathbb{x}_{1}$ e $\mathbf{x}_{2}$ no método dos elementos finitos, é exata quando do $6^{\circ}$ grau, no entanto, dispõe-se de apenas seis valores nodais, isto é, três deslocamentos e três rotações, como mostra a Fig. 4.7. Por esta razão adotou-se uma função aproximadora de $5^{\circ}$ grau para os deslocamentos, que pode ser escrita como:

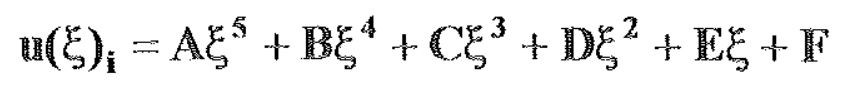

E para as rotações, tem-se: 


$$
\frac{d u_{i}}{d \xi}=\theta(\xi)_{i}=5 A \xi^{4}+4 B \xi^{3}+3 C \xi^{2}+2 D \xi+E
$$

sendo, $\mathbf{i}=\mathbf{1 , 2}$ e representa as direções $\mathbf{x}_{\mathbf{1}}$ e $\mathbf{x}_{2}$.

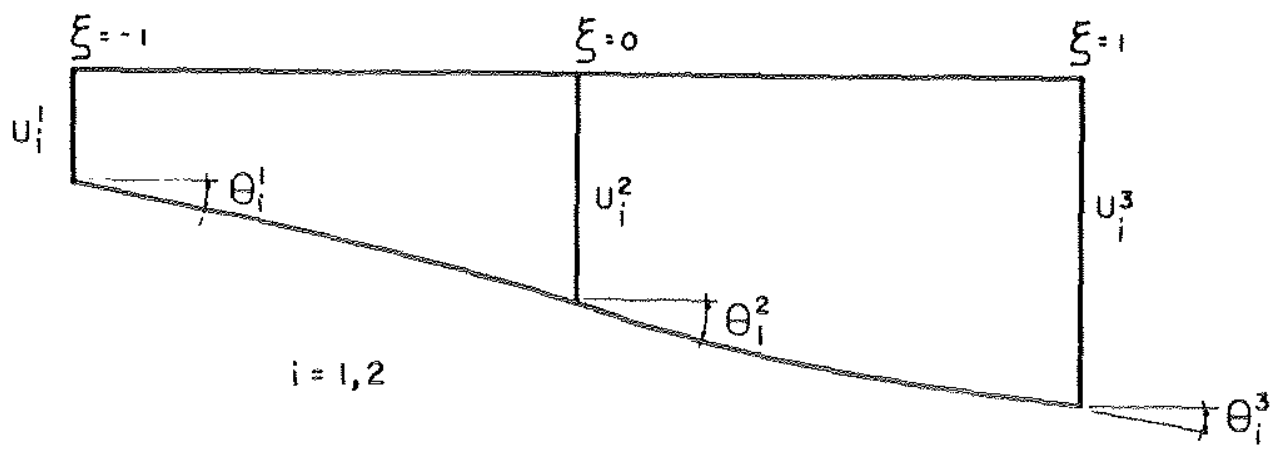

FIGURA 4.8- Valores nodais para deslocamentos e rotações nas direções $\mathrm{x}_{1} \mathrm{e}$ $\mathbf{x}_{2}$.

Os deslocamentos podem ser expressos na forma matricial como:

$$
u(\xi)=\omega \alpha
$$

sendo,

$$
\omega=\left\{\begin{array}{llllll}
\xi^{5} & \xi^{4} & \xi^{3} & \xi^{2} & \xi & 1
\end{array}\right\}
$$

Das condições de deslocamentos e rotações impostas nos pontos nodais, pode-se escrever para as direções $\mathbb{x}_{1}$ e $\mathbb{x}_{2}$ : 
$U_{i}^{n}=\left\{\begin{array}{c}U_{i}^{1} \\ \theta_{i}^{1} \frac{\ell}{2} \\ U_{i}^{2} \\ \theta_{i}^{2} \frac{\ell}{2} \\ U_{i}^{3} \\ \theta_{i}^{3} \frac{\ell}{2}\end{array}\right\}=\left[\begin{array}{cccccc}-1 & 1 & -1 & 1 & -1 & 1 \\ 5 & -4 & 3 & -2 & 1 & 0 \\ 0 & 0 & 0 & 0 & 0 & 1 \\ 0 & 0 & 0 & 0 & 1 & 0 \\ 1 & 1 & 1 & 1 & 1 & 1 \\ 5 & 4 & 3 & 2 & 1 & 0\end{array}\right]\left\{\begin{array}{l}A \\ B \\ C \\ D \\ E \\ F\end{array}\right\} \quad i=1,2$

Que matricialmente pode ser escrito como:

$\mathrm{U}^{\mathbf{n}}=\underset{\sim}{\mathrm{N} \alpha}$

Podendo-se escrever o vetor $\alpha$ como:

$\alpha=\mathbb{N}^{-1} U^{n}$

Substituindo-se a expressão (4.80) na expressão (4.76), obtém-se:

$u(\xi)=\underset{\sim}{\omega} \mathbb{N}_{\sim}^{-1} \mathbb{U}^{n}$

sendo que, a matriz $\mathrm{N}^{-1}$, apresenta os seguintes valores:

$\mathbb{N}^{-1}=\frac{1}{4}\left[\begin{array}{cccccc}-3 & 1 & 0 & 4 & -3 & 1 \\ -2 & -1 & 4 & 0 & -2 & 1 \\ -5 & -1 & 0 & -8 & 5 & -1 \\ 4 & 1 & -8 & 0 & 4 & -1 \\ 0 & 0 & 0 & 4 & 0 & 0 \\ 0 & 0 & 4 & 0 & 0 & 0\end{array}\right]$

Os deslocamentos e rotações para as direções $x_{1}$ e $x_{2}$, podem então ser escritos como: 


$$
\mathbf{u}(\xi)_{i}=\frac{1}{4}\left\{\begin{array}{c}
3 \xi^{5}-2 \xi^{4}-5 \xi^{3}+4 \xi^{2} \\
\ell / 2\left(\xi^{5}-\xi^{4}-\xi^{3}+\xi^{2}\right) \\
4 \xi^{4}-8 \xi^{2}+4 \\
\ell / 2\left(4 \xi^{5}-8 \xi^{3}+4 \xi\right) \\
-3 \xi^{5}-2 \xi^{4}+5 \xi^{3}+4 \xi^{2} \\
\ell / 2\left(\xi^{5}+\xi^{4}-\xi^{3}-\xi^{2}\right)
\end{array}\right\}\left\{\begin{array}{c}
\mathbf{U}_{i}^{1} \\
\theta_{i}^{1} \\
\mathbf{U}_{i}^{2} \\
\theta_{i}^{2} \\
\mathbf{U}_{i}^{3} \\
\theta_{i}^{3}
\end{array}\right\} \quad \mathbf{i = 1 , 2}
$$

Para a direção $x_{3}$, a função aproximadora exata seria do quarto grau, no entanto, dispõe-se de apenas três deslocamentos nodais por elemento, e por esta razão, adotou-se uma função aproximadora parabólica, representando-se os deslocamentos nessa direção da seguinte forma:

$$
\mathbf{u}(\xi)_{3}=\mathrm{G} \xi^{2}+\mathrm{H} \xi+\mathrm{I}
$$

Das condições de deslocamentos impostas nos pontos nodais, têm-se para a direção $x_{3}$ :

$$
U_{3}^{n}=\left\{\begin{array}{l}
U_{3}^{1} \\
U_{3}^{2} \\
U_{3}^{3}
\end{array}\right\}=\left[\begin{array}{ccc}
1 & -1 & 1 \\
0 & 0 & 1 \\
1 & 1 & 1
\end{array}\right]\left\{\begin{array}{c}
G \\
I \\
I
\end{array}\right\}
$$

A inversa da matriz $\mathbb{N}$, escrita apenas para a direção $\mathbb{X}_{3}$, será:

$$
N^{-1}=\frac{1}{2}\left[\begin{array}{ccc}
1 & -2 & 1 \\
-1 & 0 & 1 \\
0 & 2 & 0
\end{array}\right]
$$

Então, os deslocamentos na direção $\mathbb{3} 3$, podem ser expressos como:

$$
\mathrm{u}(\xi)_{3}=\frac{1}{2}\left\{\begin{array}{c}
\xi^{2}-\xi \\
2\left(-\xi^{2}+1\right) \\
\xi^{2}+\xi
\end{array}\right\}^{\mathrm{T}}\left\{\begin{array}{c}
\mathrm{U}_{3}^{1} \\
U_{3}^{2} \\
\mathrm{U}_{3}^{3}
\end{array}\right\}
$$

A expressão geral para os deslocamentos nas três direções, assim, ganha as seguintes forma: 


$$
\mathbf{u}(\xi)=\frac{1}{4}\left[\begin{array}{ccccccccc}
\chi^{1} & 0 & 0 & \chi^{2} & 0 & 0 & \chi^{3} & 0 & 0 \\
0 & \chi^{1} & 0 & 0 & \chi^{2} & 0 & 0 & \chi^{3} & 0 \\
0 & 0 & \chi^{4} & 0 & 0 & \chi^{5} & 0 & 0 & \chi^{6}
\end{array}\right]\left\{\begin{array}{c}
U_{1}^{1} \\
U_{2}^{1} \\
U_{3}^{1} \\
U_{1}^{2} \\
U_{2}^{2} \\
U_{3}^{2} \\
U_{1}^{3} \\
U_{2}^{3} \\
U_{3}^{3}
\end{array}\right\}
$$

ou,

$$
\mathbf{u}(\xi)=\chi U^{\mathbf{n}}
$$

sendo que:

$$
\begin{aligned}
& \chi^{1}=3 \xi^{5}-2 \xi^{4}-5 \xi^{3}+4 \xi^{2} \\
& \chi^{2}=4 \xi^{4}-8 \xi^{2}+4 \\
& \chi^{3}=-3 \xi^{5}-2 \xi^{4}+5 \xi^{3}+4 \xi^{2} \\
& \chi^{4}=2\left(\xi^{2}-\xi\right) \\
& \chi^{5}=4\left(1-\xi^{2}\right) \\
& \chi^{6}=2\left(\xi^{2}+\xi\right)
\end{aligned}
$$

Após efetuarem-se as integrações, a matriz $\mathbb{T}$, para um determinado elemento $\mathbf{j}$, pode ser escrita como:

$$
T=\frac{1}{210} \frac{\ell}{2}\left[\begin{array}{ccccccccc}
57 & 0 & 0 & 44 & 0 & 0 & -3 & 0 & 0 \\
0 & 57 & 0 & 0 & 44 & 0 & 0 & -3 & 0 \\
0 & 0 & 56 & 0 & 0 & 28 & 0 & 0 & -14 \\
16 & 0 & 0 & 192 & 0 & 0 & 16 & 0 & 0 \\
0 & 16 & 0 & 0 & 192 & 0 & 0 & 16 & 0 \\
0 & 0 & 28 & 0 & 0 & 224 & 0 & 0 & 28 \\
-3 & 0 & 0 & 4 & 0 & 0 & 57 & 0 & 0 \\
0 & -3 & 0 & 0 & 44 & 0 & 0 & 57 & 0 \\
0 & 0 & -14 & 0 & 0 & 28 & 0 & 0 & 56
\end{array}\right]
$$


A matriz $\mathbf{T}$ pode ainda ser determinada, admitindo-se dois elementos consecutivos, cada um de comprimento $\ell / 2$. Utilizando-se as condições de equilíbrio e de contorno, em um procedimento, sem aproximações, semelhante ao utilizado em análise matricial, determinam-se as ações nodais equivalentes em cada nó e em cada uma das direções principais, obtendo-se a matriz $\mathbf{T}$, que está abaixo representada.

$$
\mathrm{T}=\frac{1}{240} \frac{\ell_{j}}{2}\left[\begin{array}{ccccccccc}
74 & 0 & 0 & 56 & 0 & 0 & -10 & 0 & 0 \\
0 & 74 & 0 & 0 & 56 & 0 & 0 & -10 & 0 \\
0 & 0 & 70 & 0 & 0 & 60 & 0 & 0 & -10 \\
16 & 0 & 0 & 208 & 0 & 0 & 16 & 0 & 0 \\
0 & 16 & 0 & 0 & 208 & 0 & 0 & 16 & 0 \\
0 & 0 & 20 & 0 & 0 & 200 & 0 & 0 & 20 \\
-10 & 0 & 0 & 56 & 0 & 0 & 74 & 0 & 0 \\
0 & -10 & 0 & 0 & 56 & 0 & 0 & 74 & 0 \\
0 & 0 & -10 & 0 & 0 & 60 & 0 & 0 & 70
\end{array}\right]
$$

\subsection{RESISTENCIA DIC PONTA}

Foi desenvolvido um procedimento que permite a consideração da resistência de pontada estaca e incorporado ao presente modelo.

A estaca pode ser discretizada em um número qualquer de nós, desde que compativeis com a função aproximadora das forças de interação, admitida parabólica. Para o último elemento da estaca, um nó intemo adicional j’é introduzido, como apresentado à Figura 4.9.

Assim, as forças de superficie o consequentemente os deslocamentos podem ser expressos em um ponto $\mathbb{S}$ qualquer (no caso $\mathfrak{j}^{9}$ ) do elemento, em termos de seus valores nodais $U^{N}$ e $\mathbb{Q}^{\mathbb{N}}$, através de três funções interpoladoras, eq. (4.5), 
obtendo-se equações adicionais, a ser incorporadas ao sistema de equações do método dos elementos de contorno.

O mesmo ocorre em relação ao sistema de equações do método dos elementos finitos, visto que, um novo elemento deve ser considerado.

Os demais procedimentos são semelhantes aos já descritos para a combinação dos métodos dos elementos de contorno e o método dos elementos fínitos.

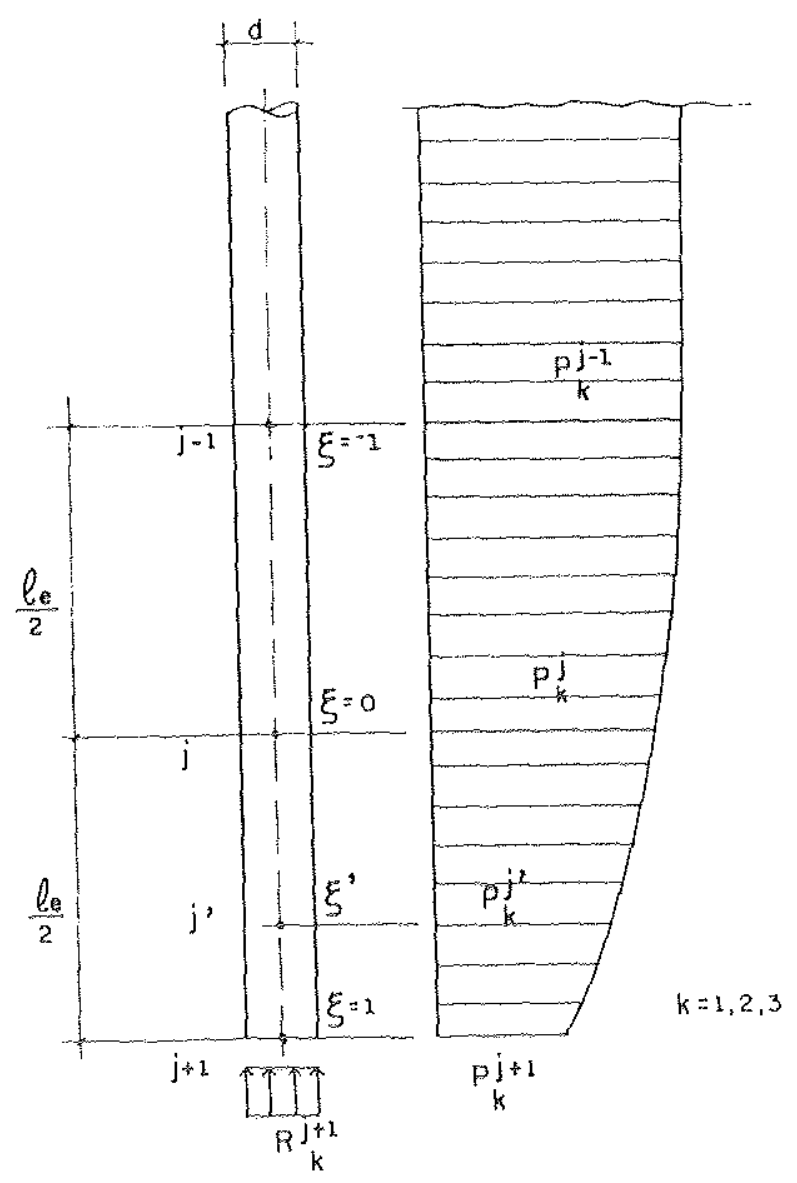

FIGURA 4.9 - Representação do último elemento de uma estaca, com indicação do nó adicional para considerar a resistência de ponta. 


\subsection{APLICAÇÕES NUMÉRICAS}

Neste item são apresentados alguns resultados obtidos a partir dos modelos formulados, além de comparações com outros modelos.

\subsubsection{Estaca vertical sob carga vertical}

Considera-se uma estaca cilíndrica vertical, em concreto, com comprimento de $8 \mathrm{~m}$ e diâmetro igual a $50 \mathrm{~cm}$, submetida a uma carga vertical de $100 \mathrm{kN}$, no topo da estaca. O módulo de deformação do concreto da estaca é igual a $20 \mathrm{GN} / \mathrm{m}^{2}$. O solo é admitido homogêneo, elástico linear, com módulo de deformação igual a 10 $\mathrm{MN} / \mathrm{m}^{2}$ e coeficiente de Poisson ( $v$ ) igual a $0,5$.

Neste exemplo a estaca foi discretizada com apenas três nós. Comparam-se os resultados do presente modelo, como obtidos usando-se os procedimentos indicados por POULOS (1968), discretizando-se a estaca com quatro nós e admitindo-se as forças de superficie constantes em cada elemento.

Os resultados em termos de deslocamentos e forças de superficie são apresentados, respectivamente, nas Figura 4.10 e Figura 4.11. Observa-se uma boa concordância entre os resultados obtidos a partir da presente análise e a proposta de POULOS. 


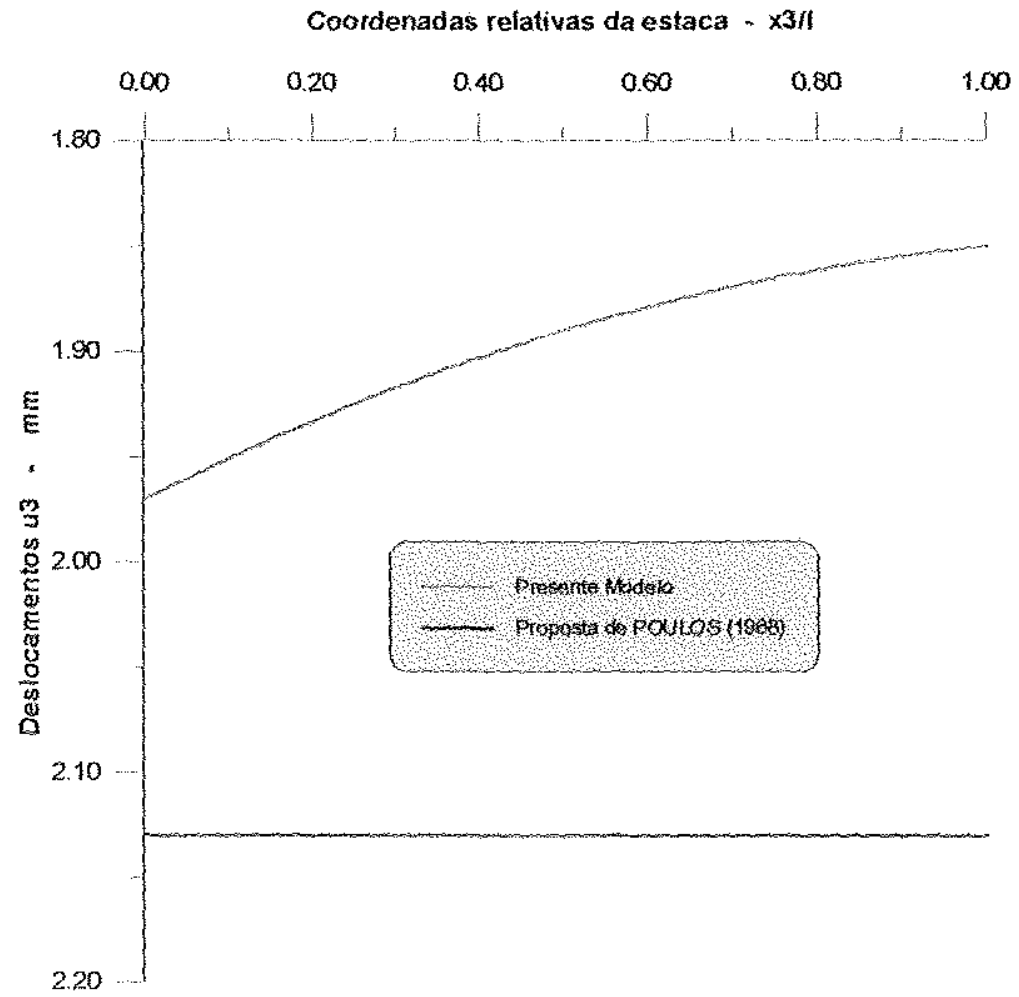

FIGURA 4.10-Deslocamentos ao longo da estaca na direção $\times_{3}$.

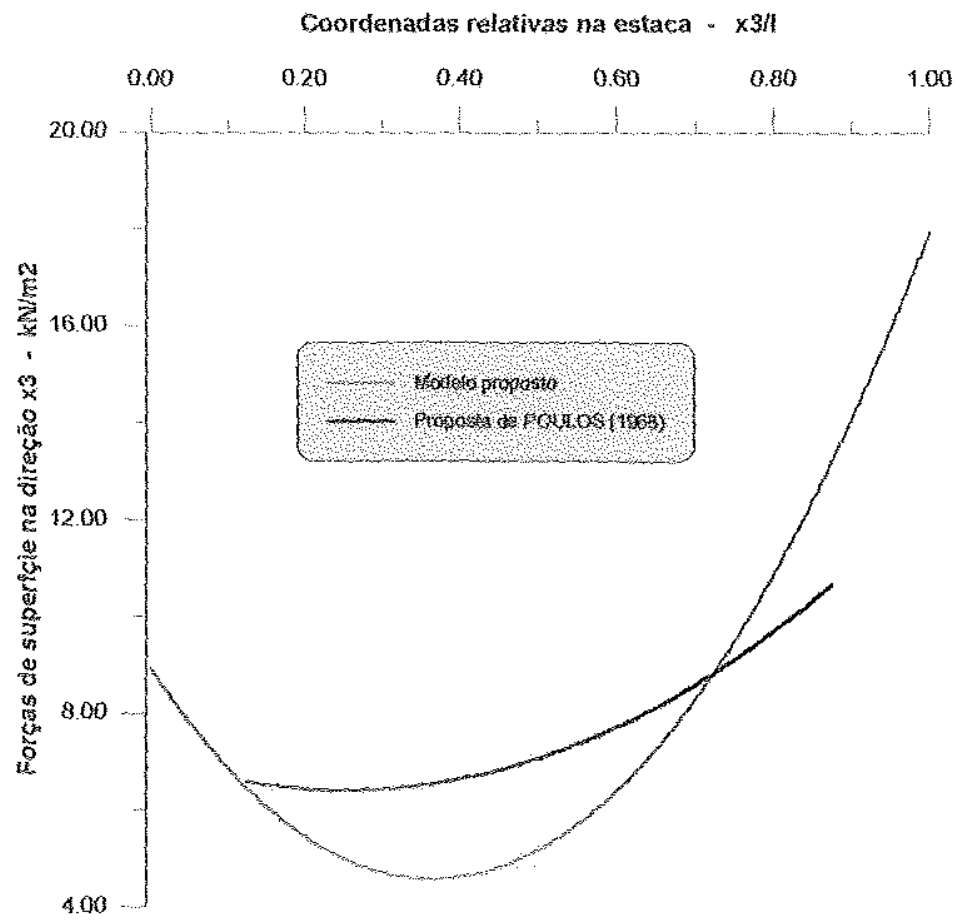

FIGURA 4.11 - Forças de superficie ao longo da estaca na direção 3 . 
O mesmo exemplo foi empregado para considerar a resistência de ponta. No presente modelo foi adicionado um nó, localizado na cota $x_{3}=6 \mathrm{~m}$, enquanto na configuração proposta por POULOS, foi acrescentado um nó na extremidade inferior da estaca.

Nas Figura 4.12 e Figura 4.13 são apresentados os resultados das duas formulações, em termos de deslocamentos na direção $\mathbf{x}_{3}$ e forças de superfície ao longo do comprimento da estaca, também na direção $\mathbf{x}_{3}$. Novamente, observa-se uma boa concordância dos resultados obtidos a partir dos dois procedimentos apresentados.

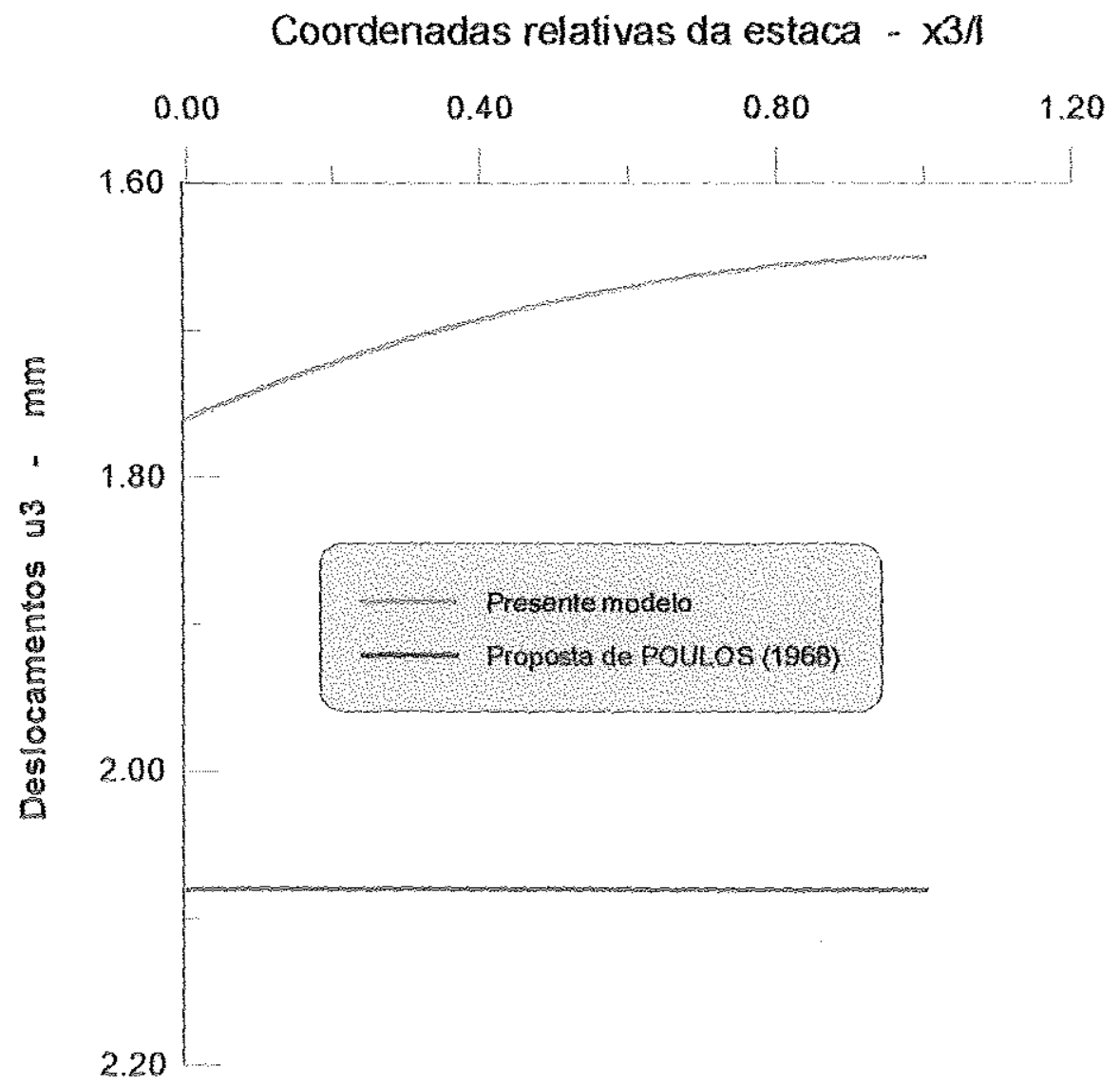

FIGURA 4.12 - Deslocamentos ao longo da estaca na direção $\mathbb{x}_{3}$, considerando-se a resistência de ponta. 


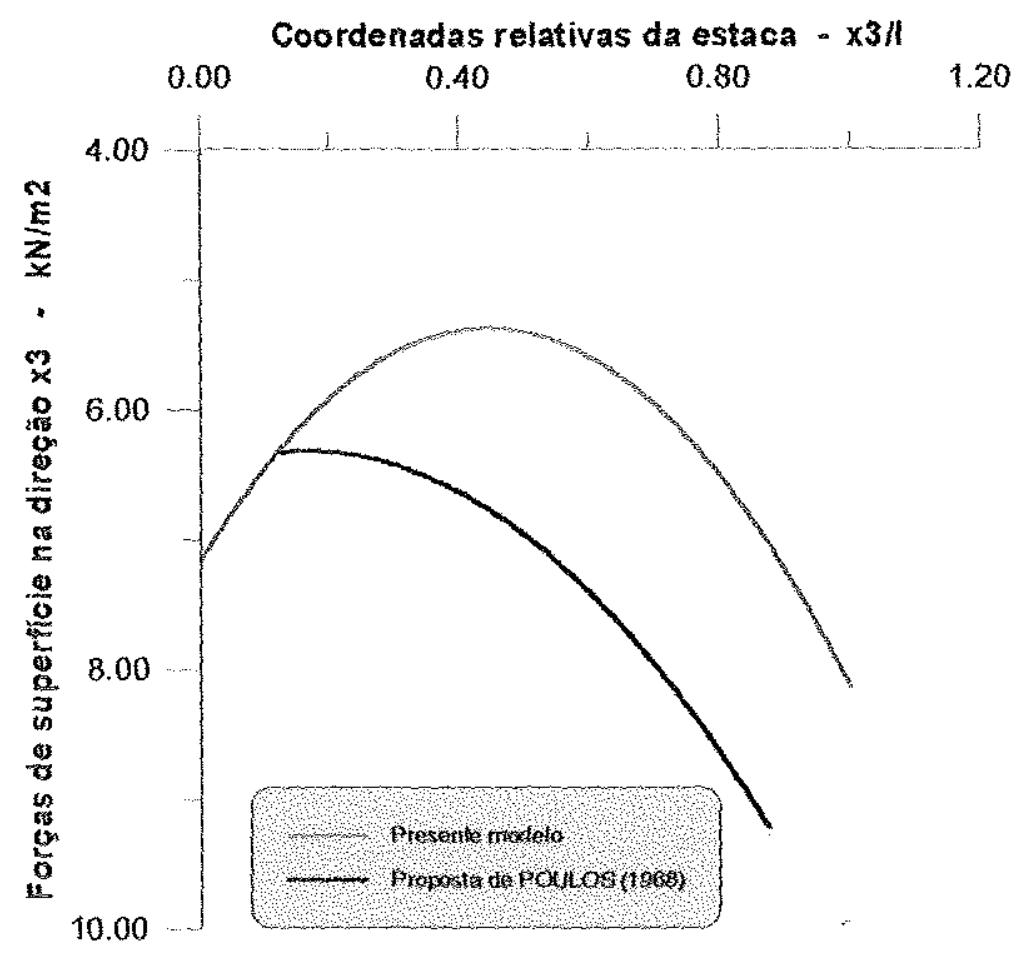

Figura 4.13 - Forças de superficie ao longo da estaca na direção $\mathbf{x}_{3}$, considerando-se a resistencia de ponta.

\subsubsection{Imteração entre duas estacas sob carga vertical}

O acréscimo de deslocamento ventical em uma estaca em meio semi-infinito, tridimensional, devido a outra estaca idêntica e igualmente carregada, é representado por um fator de interaçăo $\alpha$, definido como:

$$
\alpha=\frac{\text { deslocamento vertical adicional devido à estaca adjacente }}{\text { deslocamento vertical da estaca isolada }}
$$

A Figura 4.14, ilustra a redução do efeito de interação, com o aumento do espaçamento entre as estacas. Note-se que o fator $\alpha$, varia não só com o espaçamento entre estacas, mas tambén com o coeficiente de Poisson $(v)$ do solo, a relação entre o módulo de deformação da estaca e o módulo de deformação do solo $\left(\kappa=\frac{\mathbb{E}_{\text {estaca }}}{\mathbb{C}_{\text {solo }}}\right)$, 
além da relação comprimento/diâmetro da estaca $\left(\frac{\ell}{\mathbf{d}}\right)$, que no presente exemplo foi mantida constante.

Pode-se observar que mesmo para grandes espaçamentos entre estacas, o valor do fator $\alpha$ ainda é significativo.

Em POULOS (1968) pode-se encontrar gráficos semelhantes aos aqui apresentados.

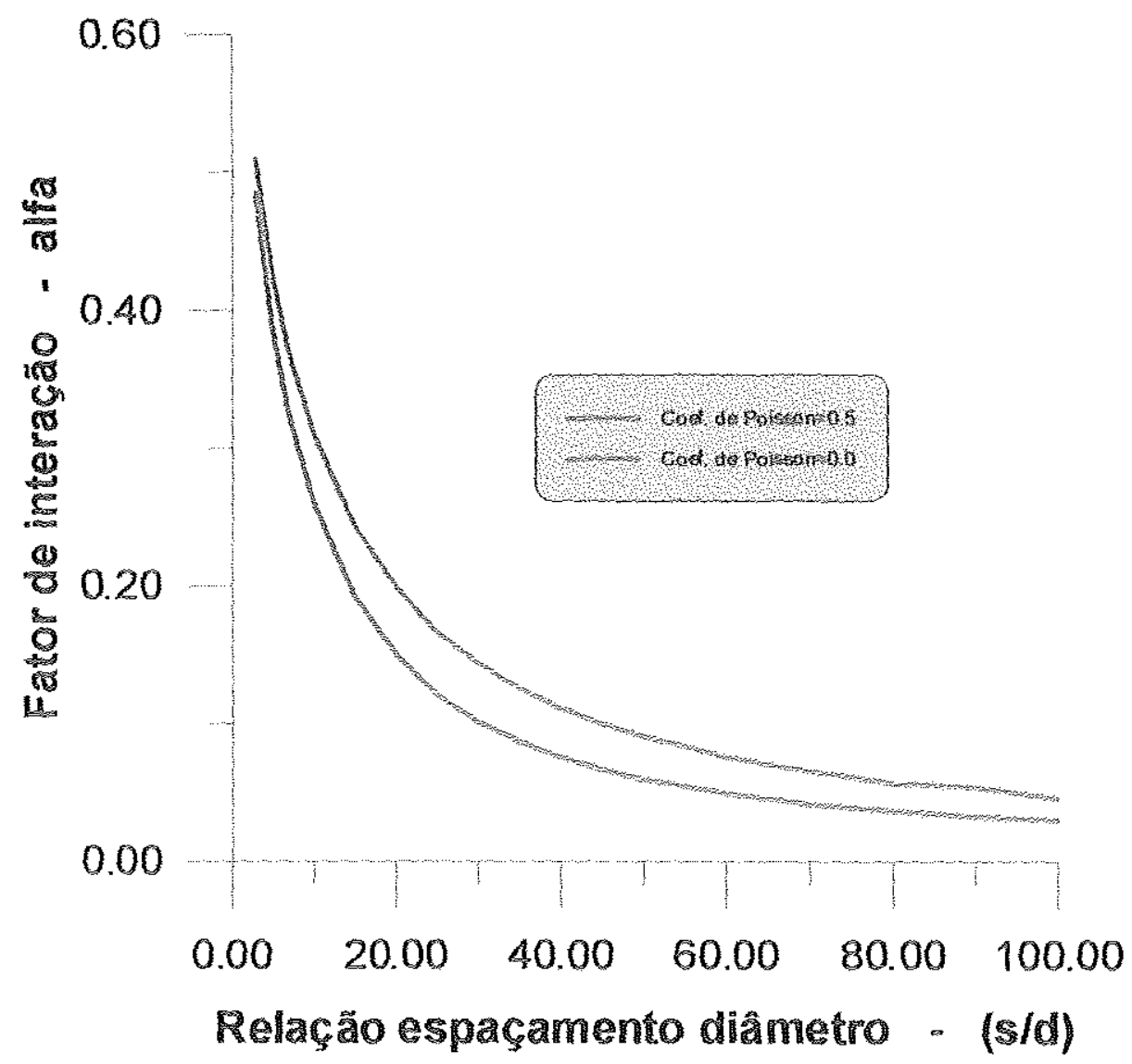

FIGURA 4.14 - Representação da interação de deslocamentos na direção \& $_{3}$ entre duas estacas verticais com cargas verticais, para $\kappa=500$. 


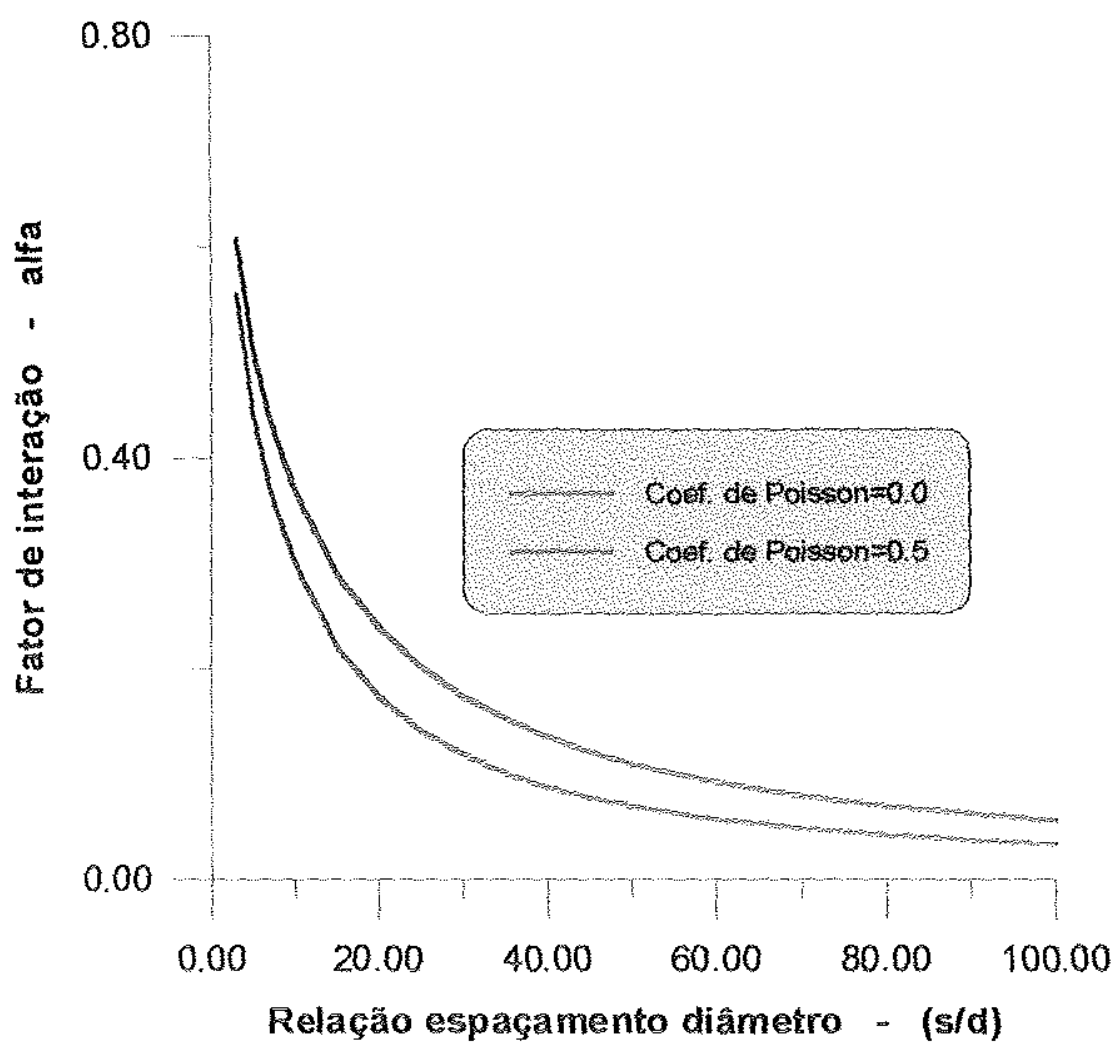

FIGURA 4.15 - Representação da interação de deslocamentos na direção $\mathbb{x}_{3}$ entre duas estacas verticais com cargas verticais, para $\kappa=100000$.

Mostra-se na Fig. 4.16 os deslocamentos horizontais em relação ao eixo $x_{1}$, das duas estacas verticais submetidas a carga vertical de $100 \mathrm{kN}$, aplicada em cada uma delas, tendo o solo coeficiente de Poisson $v=0$ e $k=500$. Pode-se observar que enquanto os topos das estacas se aproximam, suas extremidades inferiores se afastam, tendo em vista uma maior transferência de carga das estacas para o solo, próximo das pontas das estacas. $\mathrm{O}$ afastamento próximo ao topo das estacas pode ser explicado devido a rigidez das estacas. 


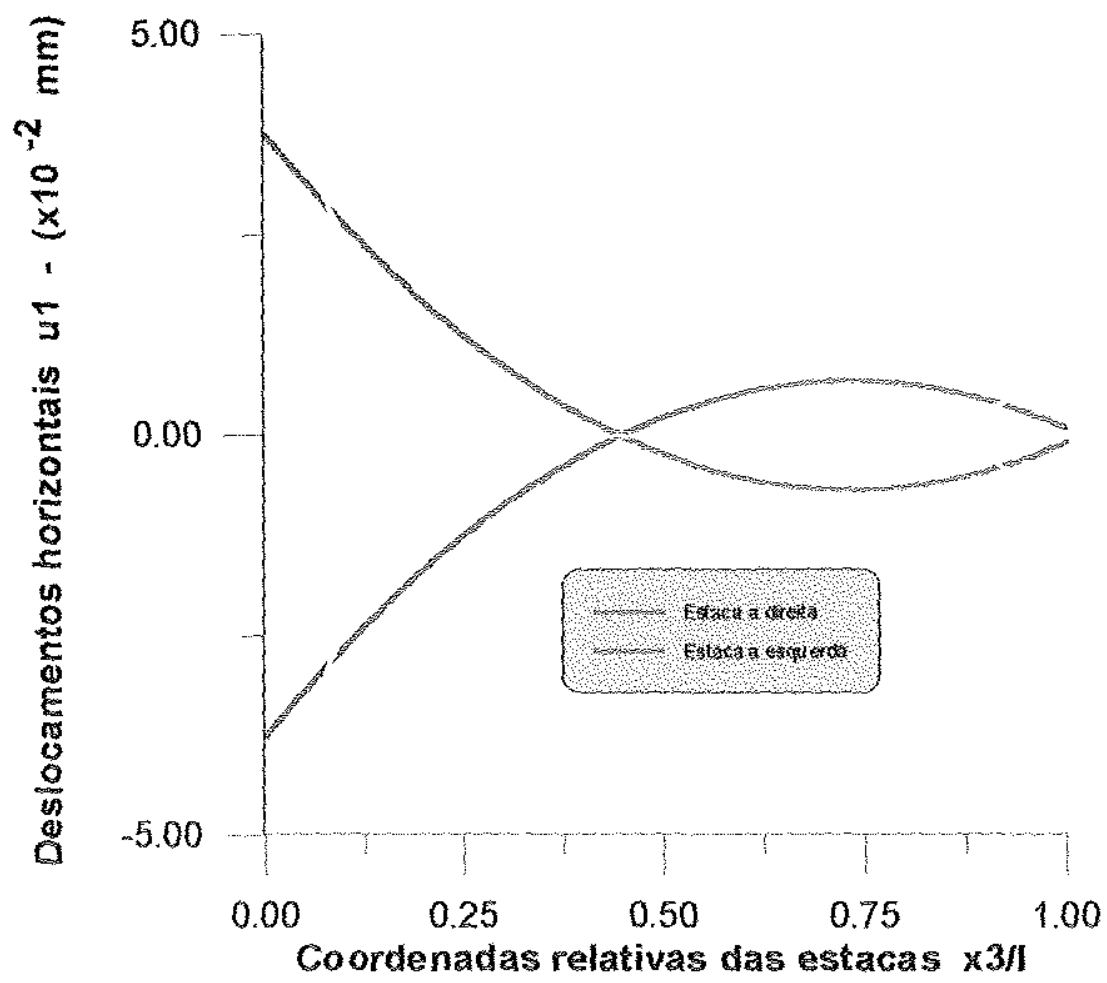

FIGURA 4.16 - Deslocamentos na direção $x_{1}$, para duas estacas sob carga vertical, $v=0$ e $\kappa=500$.

\subsubsection{Estaca inclinada sob carga vertical}

Uma estaca cilindrica em concreto, com comprimento de $8 \mathrm{me}$ módulo de deformação igual $20,7 \mathrm{GPa} / \mathrm{m}^{2}$, foi instalada no solo com uma inclinação de $1: 3$ com a vertical. No topo da estaca está aplicada uma carga vertical de $100 \mathrm{kN}$. O solo é admitido homogêneo, elástico linear, com módulo de deformação igual a 10 $\mathrm{MN} / \mathrm{m}^{2}$ e coeficiente de Poisson ( $v$ ) igual a zero.

Os deslocamentos verticais e horizontais são mostrados na Figura 4.17 e Figura 4.18 , respectivamente. 


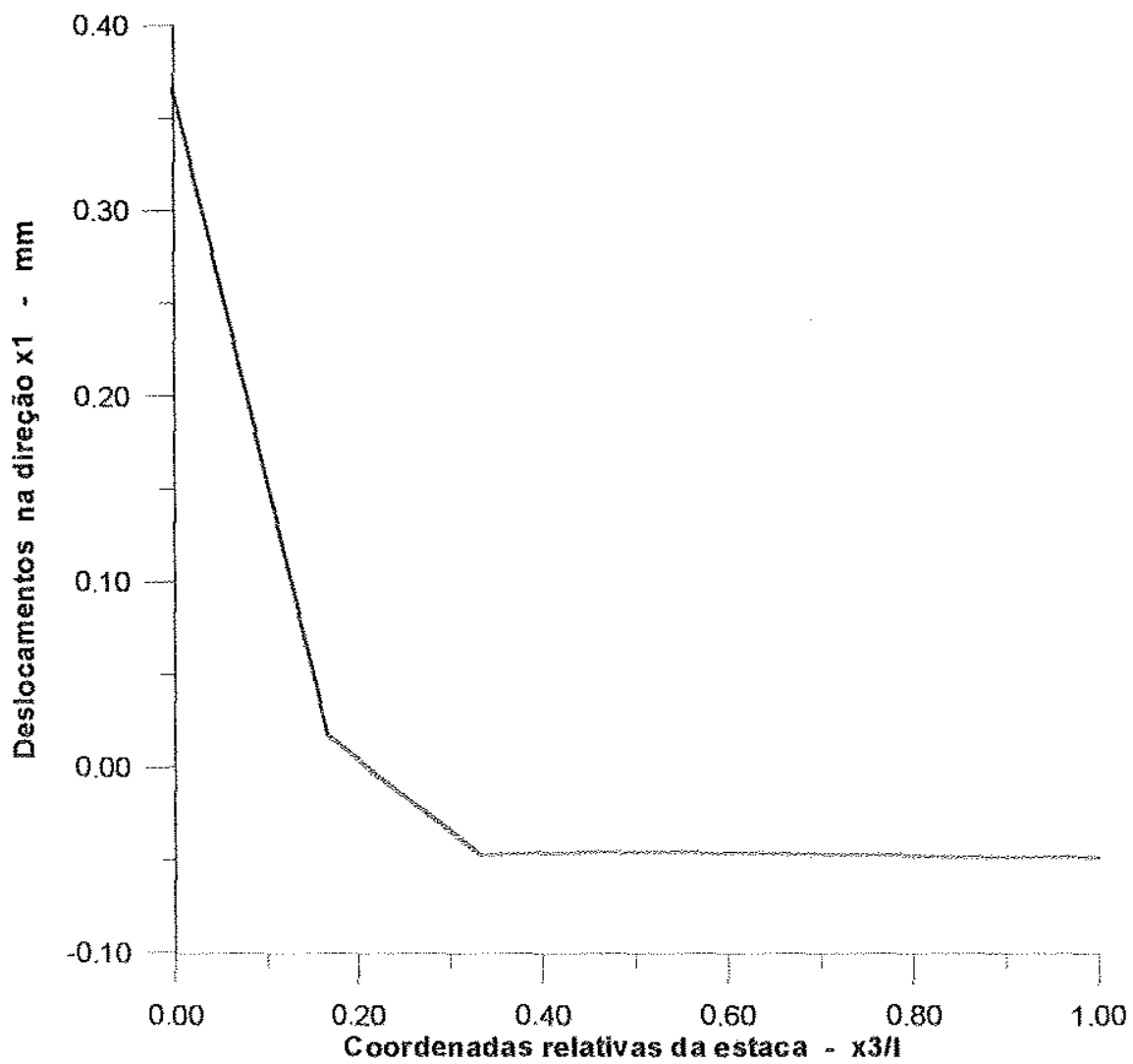

FIGURA 4.17 -- Deslocamentos horizontais na direção $\mathrm{x}_{1}$ ao longo do comprimento de uma estaca inclinada.

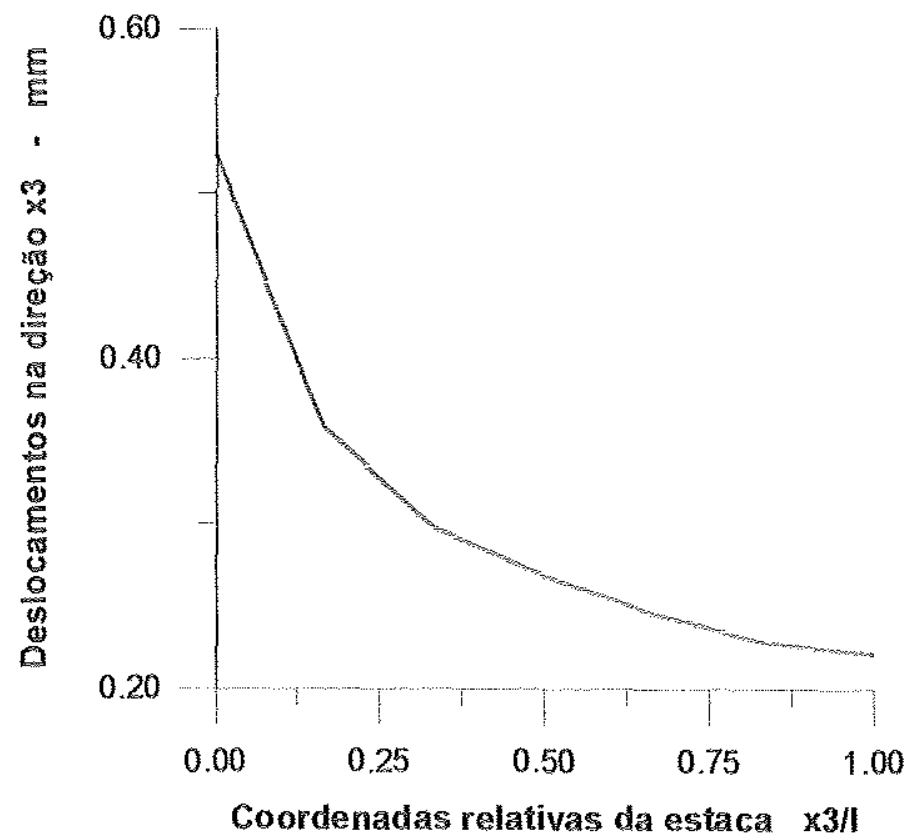

FIGURA 4.18 - Deslocamentos verticais na direção $x_{3}$ ao longo do comprimento de uma estaca inclinada. 


\subsubsection{Estaca inclinada sob carga horizontal}

Apresenta-se a seguir os resultados obtidos a partir da modelagem da mesma estaca inclinada descrita no exemplo anterior, agora sob carga horizontal de $20 \mathrm{kN}$, na direção $\mathbf{x}_{1}$ e no mesmo sentido da inclinação da estaca.

Na Figura 4.19 são mostrados os deslocamentos horizontais na direção $\mathbf{x}_{1}$, ao longo do comprimento da estaca.

$\mathrm{Na}$ Figura 4.20 representa-se a distribuição das forças de superficie horizontais na direção $\mathbf{x}_{\mathbf{1}}$, ao longo do comprimento da estaca.

\section{Coordenadas relativas da estaca $\mathrm{x} 3 / 1$}

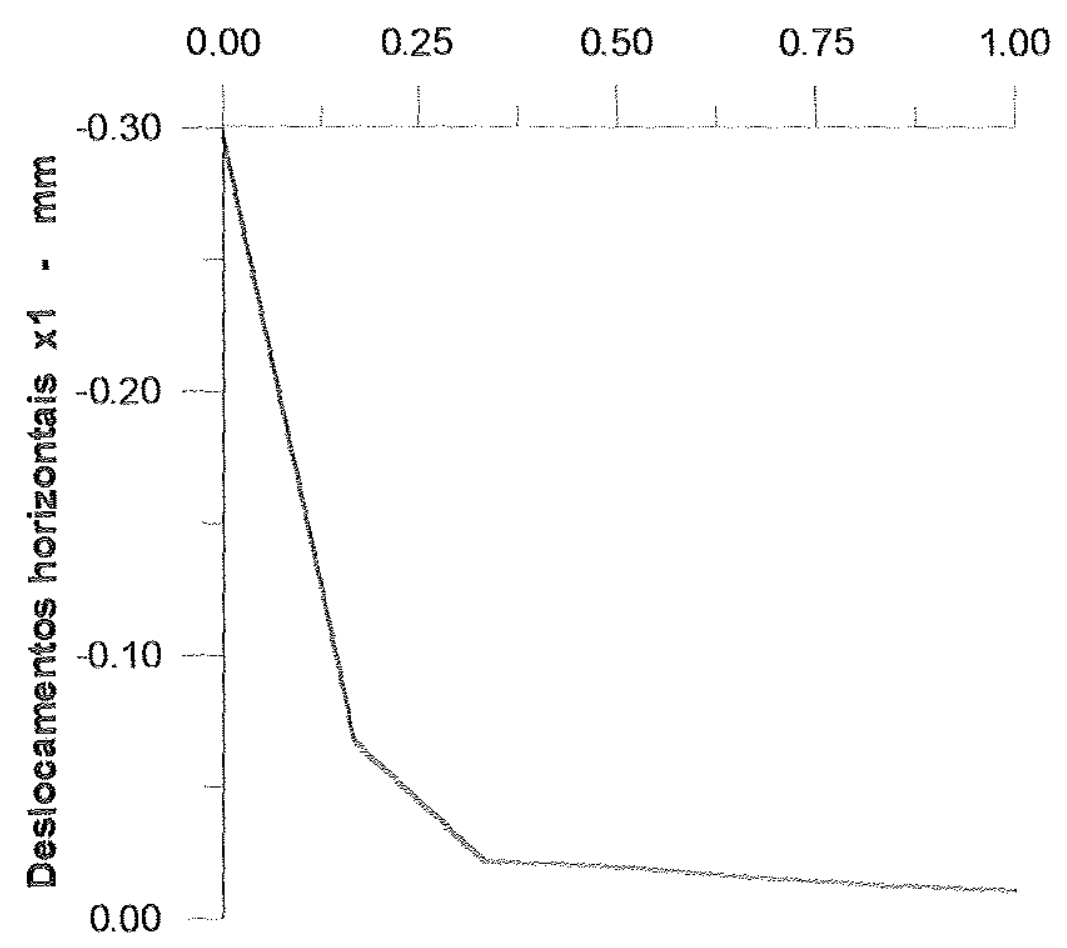

FIGURA 4.19 - Deslocamentos horizontais na direção $x_{1}$ ao longo do comprimento de uma estaca inclinada, sob a ação de uma carga horizontal. 
Coordenadas relativas da estaca $\times 3 / 1$

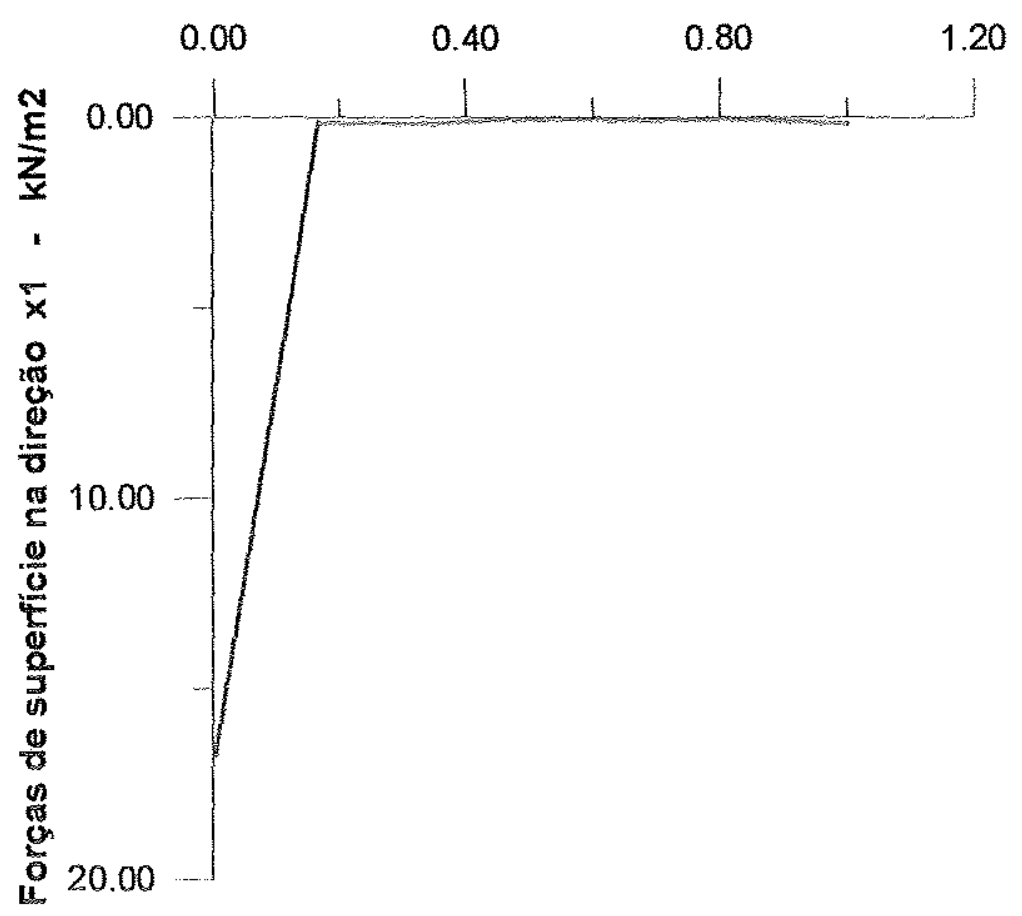

FIGURA 4.20 - Forças de superfície na direção $x_{1}$ ao longo do comprimento de uma estaca inclinada, sob a ação de uma carga horizontal. 


\section{MODELO NÃO-LINEAR PARA O ACOPLAMENTO DO SOLO A ESTACA}

\subsection{INTRODUCÃO}

Neste capítulo, estende-se a formulação anteriormente desenvolvida para considerar o comportamento não-linear na interface entre o solo e a estaca. Será utilizado um modelo elastoplástico para a modelagem do comportamento das forças interativas entre a superficie da estaca e do solo. Para tanto, apresenta-se, inicialmente, alguns conceitos da teoria da plasticidade para casos unidimensionais que sẫo a seguir generalizados para estados multidimensionais e, que govemam o comportamento elastoplástico dos materiais, bem como, são introduzidas as expressões necessárias ao equacionamento do problema.

Posteriormente, são apresentadas as hipóteses e simplificações introduzidas tendo em vista sua aplicação em problemas de interação solo-fundação em estacas, assim como, o procedimento para a solução numérica de tais problemas.

Ao final do Capítulo, alguns exemplos numéricos são analisados, considerandose o comportamento não-linear do solo, com o objetivo de se verificar a adequação da formulação apresentada ao problema proposto.

\subsection{PRINCIPIOS BASICOS DA TEORIA DA PLASTICIOAOR}

Apresenta-se, neste item, os conceitos básicos da teoria da plasticidade, utilizados na solução de problemas que apresentam não linearidade fisica. Um tratamento mais 
detalhado sobre o assunto pode ser encontrado em HILL (1950), HODGE (1959), MELDELSON (1968), CHEN (1982), dentre outros.

Basicamente, o comportamento plástico de um material fica caracterizado pelo aparecimento de deformações irreversíveis $\varepsilon^{p}$, mesmo quando se anula a solicitação a que o corpo está submetido. Essas deformações plásticas, que são consideradas independentes do tempo de aplicação do carregamento, aparecem após atingida a tensão de início de escoamento do material $\sigma_{0}$. A Fig. 5.1 mostra esquematicamente uma possível curva tensão-deformação, no caso de um ensaio uniaxial, para um material com estas caracteristicas.

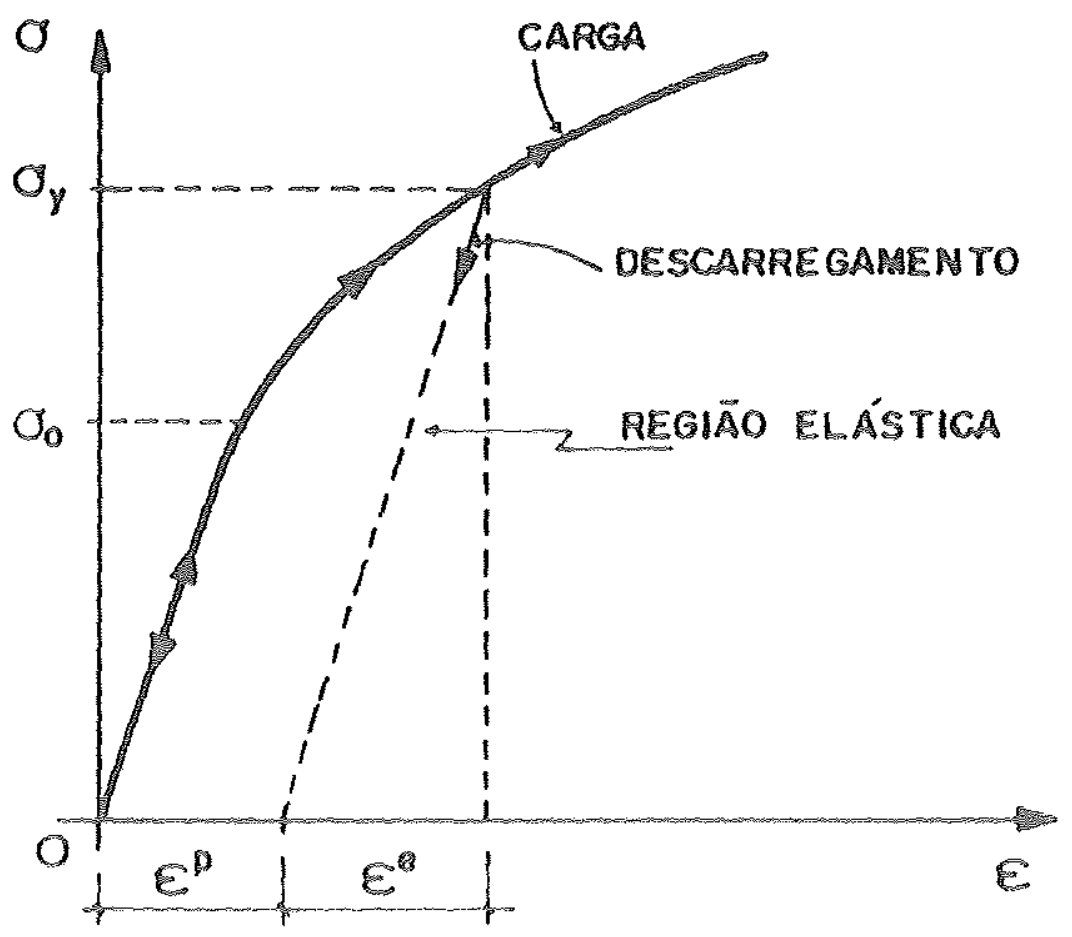

FIGURA 5.1 - Diagrama tensão-deformação para um ensaio uniaxial.

A configuração deformada é dependente do nível de tensão e da história do carregamento. Observa-se na Fig. 5.1, que, para solicitações que provocam niveis de tensão inferiores a $\sigma_{0}$, o comportamento do material é elástico, e deste modo, para sucessivas situações de carga e descarga, o caminho percorrido encontra-se sempre no 
trecho linear inicial $\overline{\mathbf{O A}}$, não revelando portanto o aparecimento de deformações permanentes ou residuais.

Entretanto, quando o corpo é solicitado por um nível de tensão superior à tensão de escoamento, caracteriza-se o comportamento plástico do material, no qual se verifica que, ao efetuar a descarga, esta não se dá pelo mesmo caminho percorrido durante a carga, mas segundo uma reta aproximadamente paralela à reta inicial ao do regime elástico. Em consequiência, para um nível de tensão nula, verifica-se o aparecimento de uma deformação irreversível, denominada deformação plástica $\varepsilon^{\mathbf{P}}$.

Constata-se que, a partir da situação atual ao efetuar um novo carregamento, este se dará segundo o mesmo caminho linear do descarregamento, até que seja atingido um novo valor para a tensão de escoamento, $\sigma_{y}$, que é função da deformação plástica acumulada até então. Este fenômeno é denominado de endurecimento ou encruamento do material.

Para que se obtenha uma formulação de uma teoria que permita modelar o comportamento elastoplástico de um material, devem ser conhecidas relações explícitas entre tensão e deformação que descrevam este comportamento em regime elástico, e também, outras relações que representem o comportamento do material pósescoamento, que caracteriza o regime plástico. Finalmente, precisa também ser conhecido, um critério de escoamento que indique o nível de tensão a partir do qual surgem deformações plásticas.

\subsection{PROBLEMAS ELASTOPLÁSTICOS UNTOMMUNSIONAIS}

Para situações unidimensionais, o modelo é facilmente definido apenas em função da curva tensão-deformação, obtida a partir de ensaio uniaxial de um corpo de prova do material em estudo. Usualmente, algumas curvas tensão-deformação idealizadas são admitidas para representar o comportamento do material, objetivando simplificar a análise elastoplástica.

A idealização mais simples corresponde ao comportamento elastoplástico perfeito, e que é a adotada no presente trabalho, já que conduz a resultados satisfatórios 
para a análise elastoplástica dos problemas que aqui serão tratados. $\mathrm{O}$ material, cujo o diagrama tensão-deformação está apresentado na Fig. 5.2, tem comportamento puramente elástico para níveis de tensões inferiores a tensão de escoamento $\sigma_{0}$. Entretanto, quando a tensão é igual a tensão de escoamento $\sigma_{0}$, verifica-se o aparecimento de deformações plástica $\varepsilon^{\mathbf{p}}$.

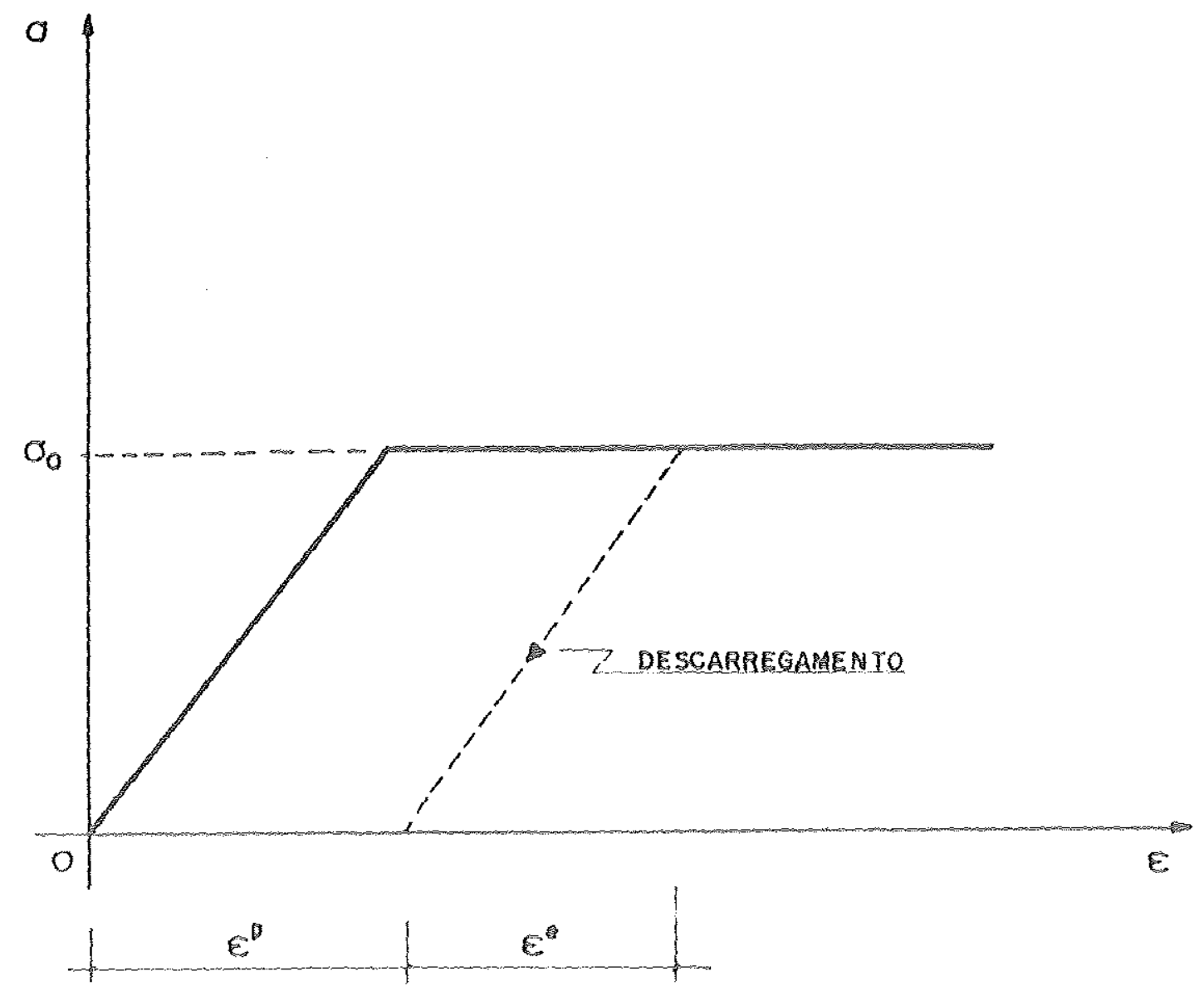

FIGURA 5.2 - Diagrama tensão deformação para material elastoplástico perfeito

A deformação total, devida a qualquer estado de carregamento, pode ser decomposta em uma componente elástica, $\varepsilon^{\mathrm{e}}$, e em uma componente plástica, $\varepsilon^{\mathrm{p}}$, isto $e^{\text {: }}$

$$
\varepsilon=\varepsilon^{e}+\varepsilon^{p}
$$

O nivel de tensão para o material elastoplástico perfeito está limitado à seguinte condição: 


$$
\sigma-\sigma_{0} \leq \mathbf{0}
$$

Um outro comportamento consiste en assumir uma curva bilinear, apresentada na Fig. 5.3, com endurecimento linear do material após o escoamento. Neste caso, para tensões abaixo da tensão inicial de escoamento $\sigma_{\mathbf{a}}$, (ponto $\mathbf{A}$ da curva), 0 comportamento do material é elástico e seu módulo de elasticidade igual a $\mathbf{E}$. Atingida a tensão de escoamento, um incremento de tensão acarreta um incremento de deformação, com o aparecimento de deformações plásticas , $\varepsilon^{\mathrm{p}}$, segundo uma inclinação denominada de módulo tangente $\mathbf{E}_{\mathbf{T}}$. assim, após o escoamento inicial, conforme mostra a Fig. 5.3, tem-se:

$$
d \sigma=\mathrm{C}_{\mathrm{T}} \mathrm{d} \varepsilon
$$

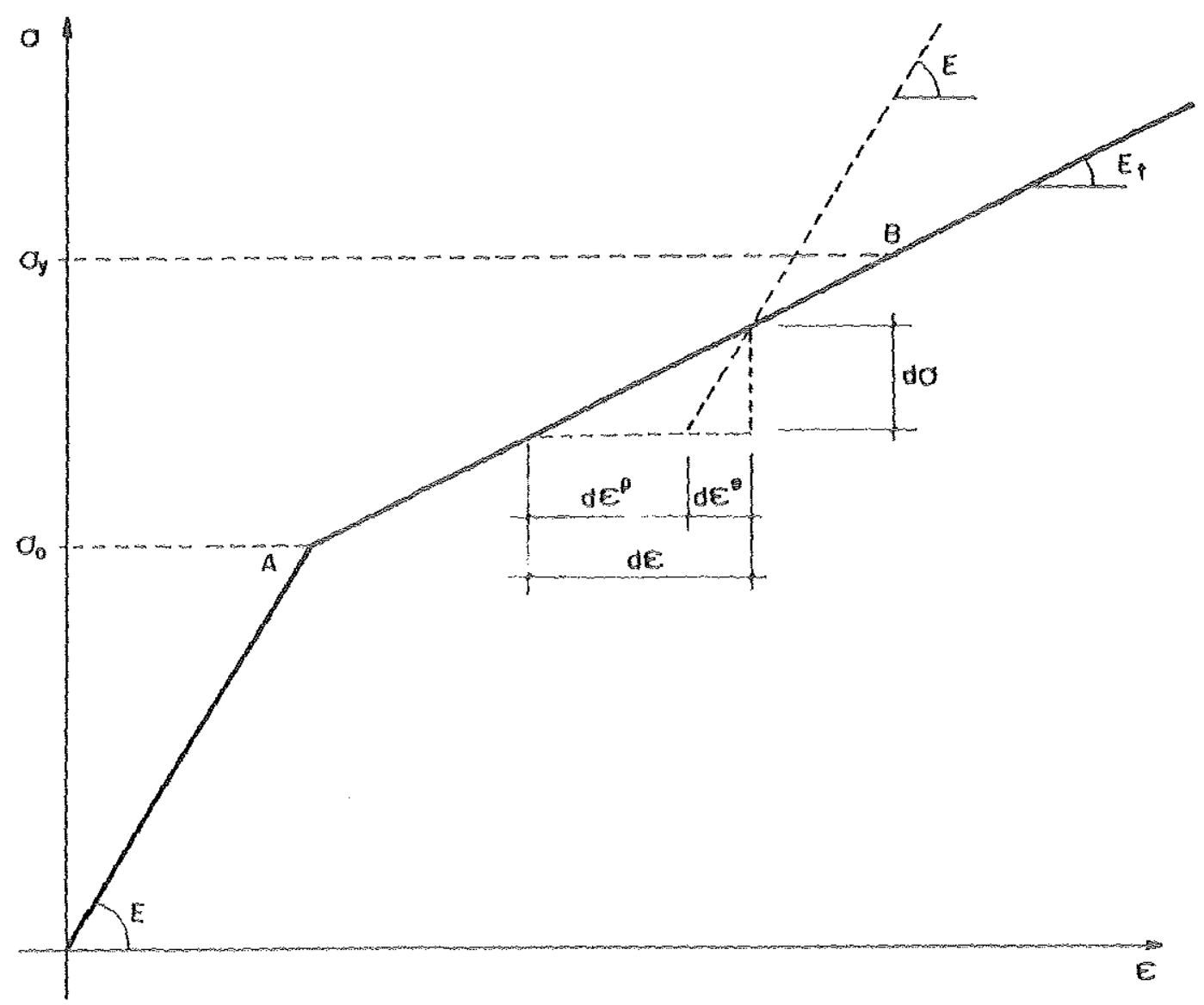

FIGURA 5.3 - Diagrama tensão-deformaçâo para um material elastoplástico com endurecimento linear. 
Com o incremento de deformação total $\mathbf{d} \varepsilon$, podendo ser decomposto em uma componente elástica e outra plástica, ou seja:

$$
\mathbf{d} \varepsilon=\mathbf{d} \varepsilon^{\mathbf{e}}+\mathbf{d} \varepsilon^{p}
$$

A partir da observação da Fig. 5.3, pode-se constatar que, mesmo após o início do escoamento, incrementos de tensão correspondem a incrementos de deformação elástica, dada por:

$$
\mathbf{d} \sigma=\mathbb{E} \mathbf{d} \varepsilon^{\mathrm{e}}
$$

No caso do material em estudo que apresenta curva tensão-deformação bilinear, define-se o parâmetro H', denominado de parâmetro de endurecimento, como a relação entre os incrementos de tensão que excedem a tensão de escoamento e os correspondentes incrementos de deformação plástica, isto é:

$$
{ }^{\prime}=\frac{d \sigma}{d \varepsilon^{p}}
$$

ou seja,

$$
H^{p}=\frac{d \sigma}{d \varepsilon-d \varepsilon^{\mathrm{e}}}
$$

Substituindo-se as eq. (5.3) e (5.5) na eq. (5.7), resulta:

$$
\mathrm{H}^{\mathrm{P}}=\frac{\mathrm{E}_{\mathrm{T}}}{\mathrm{L}-\mathrm{C}_{\mathrm{T}} \mathrm{C}}
$$

A tensão de escoamento $\sigma_{\mathbf{y}}$, em um ponto qualquer $B$, pode ser escrita em função da tensão de início de escoamento, somada aos incrementos de tensão entre os pontos $\mathrm{A}$ e $\mathrm{P}$, como:

$$
\sigma_{y}=\sigma_{0}+\int_{A}^{B} d \sigma
$$

Pode-se verificar que os incrementos de tensão estão relacionados à deformação plástica ocorrida. Assim sendo, substituindo-se a eq. (5.6) na eq. (5.9), tem-se:

$$
\sigma_{y}=\sigma_{0}+\int_{A}^{B} H^{\prime} d \varepsilon^{p}
$$

que, após efetuada a correspondente integração, resulta em: 


$$
\sigma_{y}=\sigma_{0}+H^{\prime} \varepsilon^{p}
$$

sendo $\varepsilon^{p}$ a deformação plástica acumulada no intervalo $\overline{\mathbf{A B}}$

O nível de tensão para o material elastoplástico descrito está limitado a seguinte condição:

$$
\sigma-\sigma_{y} \leq 0
$$

O primeiro membro desta inequação pode ser entendido como uma função de escoamento que não pode assumir valores positivos, ou seja:

$$
\mathbb{F}(\sigma, k) \leq \mathbf{0}
$$

ou,

$$
\sigma-Y(k) \leq 0
$$

Comparando-se as expressões (5.12) e (5.14), constata-se que Y(k) é uma função que representa a tensão de escoamento atual, $\sigma_{y}$. Portanto, pode-se verificar, a partir da eq. (5.11), que a funçăo $\mathbf{Y}(\mathbf{k})$ depende apenas do parâmetro $\mathbf{k}$, associado à história e ao comportamento quanto ao endurecimento do material.

A expressão $\mathbb{F}(\sigma, k)=0$, dada pela eq. (5.13), determina a superficie que limita a região elástica atual e corresponde a um ponto sobre a curva tensão-deformação.

\subsection{TLORA DA PLASTICIDADE NO RSPACO TRIDIMUNSIONAL}

A generalização da análise elastoplástica para estados multiaxiais de tensão pode ser feita por meio das hipóteses básicas da teoria da plasticidade juntamente com os conceitos para o caso unidimensionais apresentados anteriormente.

No meio contínuo, as relações entre tensão e deformação podem ser estabelecidas através das suas componentes tensoriais $\sigma_{\mathrm{ij}}$ e $\varepsilon_{\mathrm{ij}}$. Assim, os incrementos de deformação total $\mathrm{d} \varepsilon_{\mathbf{i j}}$, de forma análoga ao caso uniaxial, podem ser expressos através das componentes elásticas e plásticas, ou seja: 


$$
\mathrm{d} \varepsilon_{\mathrm{ij}}=\mathrm{d} \varepsilon_{\mathrm{ij}}^{\mathrm{e}}+\mathrm{d} \varepsilon_{\mathrm{ij}}^{\mathrm{p}}, \quad \mathrm{i}, \mathrm{j}=1,2,3
$$

A relação tensão-deformação, que descreve o comportamento do material na fase elástica, é obtida da eq. (2.8) e expressa como:

$$
\sigma_{i j}=C_{i j k 1} \varepsilon_{k 1}^{e}
$$

onde $\mathbb{C}_{\mathrm{ijkl}}$ é o tensor de quarta ordem das constantes elásticas, que para materiais isotrópicos, é dado por:

$$
\mathrm{C}_{\mathrm{ijkl}}=\lambda \delta_{\mathrm{ij}} \delta_{\mathrm{k} 1}+\mu \delta_{\mathrm{ik}} \delta_{\mathrm{jl}}+\mu \delta_{\mathrm{il}} \delta_{\mathrm{jk}}
$$

sendo $\lambda$ e $\mu$ as constantes de Lamé e $\delta_{\mathrm{ij}}$ o delta de Kronecker.

Para estados de tensão multiaxiais, um critério de escoamento determina o estado de tensão para o qual iniciam-se as deformações plásticas e pode ser escrito, genericamente, sob a forma:

$$
\mathbb{F}\left(\sigma_{\mathrm{ij}}, \mathrm{k}\right)=\mathbf{f}\left(\sigma_{\mathrm{ij}}\right)-\mathrm{Y}(\mathrm{k})=\mathbf{0}
$$

sendo que, $f\left(\sigma_{i j}\right)$ é a função do estado de tensão atual $\sigma_{i j}$, podendo ser interpretada como uma tensão efetiva ou equivalente uniaxial $\bar{\sigma}$. A função $\mathbf{Y}(\mathbf{k})$ depende apenas do parâmetro de endurecimento do material, $k$, podendo ser entendido como um valor convencional da tensão de escoamento, obtida a partir de ensaios uniaxiais.

O critério de escoamento definido pela função pode ser representado no espaço das tensões por uma superficie, denominada de superficie de escoamento, a qual, separa os estados de tensões elásticas daqueles que geram deformações permanentes ou plásticas.

No espaço de tensões, o estado de tensão $\sigma_{i j}$ em um ponto do corpo é representado por um ponto no interior ou sobre a superficie.

Admitindo-se válidas as hipóteses de que o material permanece homogêneo e isótropo, os eixos coordenados no espaço tridimensional podem ser relacionados às três tensões principais, sendo possível assim uma representação tridimensional para a superficie de escoamento.

A superficie que limita a região elástica inicial, como no caso uniaxial, é função apenas do estado de tensão, e portanto, a partir da eq. (5.18), pode-se escrever: 


$$
\mathbf{F}\left(\sigma_{i j}\right)=\mathbf{0},
$$

De acordo com essa representação, sendo $\mathbf{F}=\mathbf{0}$, acréscimos de estado de tensão que conduzem a valores negativos de $\mathbf{F}$ indicam situação de descarga ou entrada no regime elástico. Acréscimos de tensão que verificam a eq. (5.19) indicam uma situação limite ou carregamento neutro. Já acréscimos de tensão que conduzem a valores positivos de $\mathbf{F}$ são ditos inadmissíveis, pois indicam o aparecimento de deformações permanentes. Nesta situação, a superfície inicial de escoamento deve evoluir no espaço das deformações de tal modo que o ponto que representa o novo estado de tensão ainda resulta sobre a superfície.

Assim, alguns modelos descrevem a evolução da superficie de escoamento no espaço das tensões. Na Fig. 5.4.a está representada a superfície de escoamento para um material plástico perfeito, sendo que a tensão de escoamento não depende da deformação plástica ocorrida anteriormente.

Para materiais que apresentam endurecimento, dois modelos descrevem tal comportamento. O primeiro denominado isotrópico, indicado na Fig. 5.4.b, admite que a superficie de escoamento evolua, mantendo a sua forma inicial, sem sofrer translação, gerando superficies subseqüentes, através de expansão uniforme da superfície inicial. Quando as superficies subseqüentes preservam a forma e orientação, mas apresentam translação no espaço das tensões, como corpo rígido, o modelo é dito cinemático, conforme ilustrado na Fig. 5.4.c. 


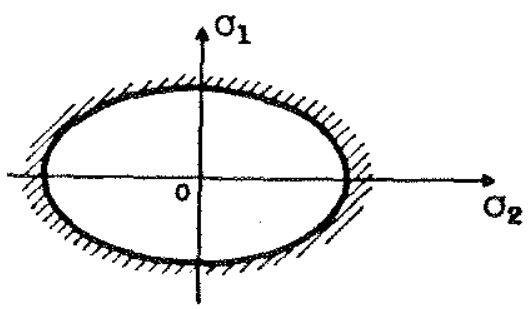

(a) PLÁstico PERFEITO

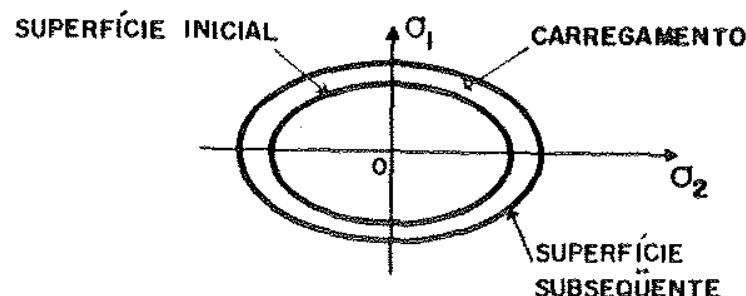

(b) ISOTRópico

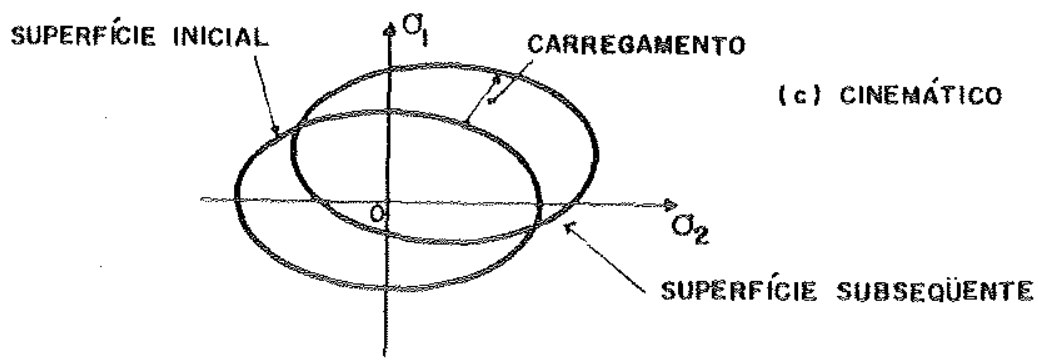

FIGURA 5.4 - Modelos de representação de comportamento com endurecimento.

No presente trabalho, o modelo para materiais plásticos perfeitos é adotado, tendo em vista que, esse modelo, pode representar satisfatoriamente o comportamento dos problemas de interação solo-fundação. No entanto, visando uma formulação mais abrangente, optou-se em apresentar o desenvolvimento matemático tendo como base o modelo com endurecimento isotrópico.

O desenvolvimento progressivo da superficie de escoamento é definido a partir da variação da função de escoamento do material $\mathbf{Y}(\mathbf{k})$, que pode ser relacionada à deformação plástica por meio do parâmetro de endurecimento k. Duas são as hipóteses admitidas para a definição deste parâmetro, A primeira, conhecida como work hardening, estabelece o parâmetro $k$ como uma função do trabalho plástico, $\mathbf{W}_{\mathbf{p}}$, acumulado durante as deformações plástica, isto é:

$$
k=W_{p}=\int \sigma_{i j} d \varepsilon_{i j}^{p},
$$

em que $d \varepsilon_{\mathrm{ij}}^{\mathrm{p}}$ é a componente da deformação plástica ocorrida durante o incremento de deformação. 
A Segunda hipótese, denominada de strain hardening, relaciona o parâmetro $\mathbf{k}$ com a medida da deformação plástica total, denominada de deformação plástica efetiva ou equivalente, $\bar{\varepsilon}^{\mathbf{p}}$, definida por:

$$
\mathbf{k}=\overline{\boldsymbol{\varepsilon}}^{\mathbf{p}}=\int \mathbf{d} \bar{\varepsilon}^{\mathbf{p}}
$$

sendo,

$$
d \bar{\varepsilon}^{p}=\sqrt{\frac{2}{3}\left(d \varepsilon_{i j}\right)^{p}\left(d \varepsilon_{i j}\right)^{p}}
$$

O estado multiaxial de tensão está associado ao estado de tensão unidimensional, por meio da tensão equivalente $\bar{\sigma} \mathbf{e}$ da deformação equivalente $\bar{\varepsilon}^{\mathbf{p}}$, que devem reproduzir a tensão $\sigma$ e a deformação plástica $\varepsilon^{\mathbf{P}}$ do ensaio uniaxial. Portanto, a relação incremental entre tensão e deformação, pode ser escrita de forma semelhante a eq. (5.6), ou seja:

$$
d \bar{\sigma}=H^{p} d \bar{\varepsilon}^{p}
$$

A partir da definição da função de escoamento, dada na eq. (5.18) e considerando-se o endurecimento do material, os estados de tensão para os quais $\uparrow<$ Y(k), caracterizam um comportamento elástico do material. Quando $\mathbf{I}=\mathbf{V}(\mathrm{k})$, atinge-se o estado plástico e uma variação incremental na função de escoamento devido a uma variação incremental de tensão, é dada por:

$$
d i=\frac{\partial r^{i}}{\partial \sigma_{i j}} d \sigma_{i j}
$$

Portanto, a partir da observação da variação da função de escoamento, podem ser caracterizadas as seguintes situações:

a) $\mathbf{d f}<\mathbf{0}$ ocorre descarregamento, caracterizado pelo comportamento elástico, e o ponto representativo do estado de tensão retorna para o interior da superficie de escoamento;

b) $\mathbf{d} \boldsymbol{f}=\mathbf{0}$ carregamento neutro, que ocorre para materiais plásticos perfeitos, permanecendo o ponto sobre a superficie de escoamento inicial, que é fixa; 
c) df $>\mathbf{0}$ carregamento plástico, no qual o material tem comportamento plástico com endurecimento, e o ponto que representa o estado de tensão permanece na superficie de escoamento em expansão.

Para a formulação de um modelo elastoplástico, é necessário estabelecer relações constitutivas que representem o comportamento do material na fase pósescoamento. Assim, faz-se uso de uma hipótese adicional da teoria de plasticidade que consiste em considerar que o incremento de deformação plástica seja proporcional ao gradiente de tensão de uma grandeza escalar denominada potencial plástico $\mathbf{Q}$, podendo-se escrever:

$$
d \varepsilon_{i j}^{p}=d \lambda \frac{\partial Q}{\partial \sigma_{i j}}
$$

sendo $\mathbf{d} \lambda$ uma constante denominada multiplicador plástico.

A eq. (5.25) é denominada regra de fluxo, uma vez que indica o fluxo plástico após o escoamento. Quando se considera o potencial plástico $\mathbf{Q}$ coincidente com a função de escoamento $\mathbb{F}\left(\sigma_{i j}, k\right)$, tem-se a regra de fluxo denominada associativa, e neste caso a eq. (5.25) pode ser escrita como:

$$
\boldsymbol{d} \varepsilon_{\mathrm{ij}}^{\mathrm{p}}=\frac{\partial \mathrm{N}\left(\sigma_{\mathrm{ij}}, \mathrm{l}\right)}{\partial \sigma_{\mathrm{ij}}}
$$

ou,

$$
\varepsilon_{\mathrm{ij}}^{\mathrm{p}}=\mathbf{d} \lambda \frac{\partial \mathrm{f}\left(\sigma_{\mathrm{ij}}\right)}{\partial \sigma_{\mathrm{ij}}}
$$

uma vez que Y(k) não é dependente do estado de tensão.

A eq. (5.26) é conhecida como condição de normalidade, uma vez que $\frac{\partial \mathrm{F}}{\partial \sigma_{\mathrm{ij}}}$ se constitui em um vetor normal à superficie de escoamento no ponto que representa o estado de tensão.

A partir da teoria da plasticidade e como conseqüência da lei associativa de escoamento, é possível demonstrar que, a condição de irreversibilidade das deformaçôes plásticas implica na realização de trabalho plástico positivo durante um ciclo completo de tensão. Este fato garante a convexidade tanto da superficie de escoamento inicial, 
quanto de todas as superficies de escoamento subseqüentes. As condições de normalidade de fluxo plástico e convexidade da superfície de escoamento são adequadas aos materiais com comportamento elastoplástico perfeito ou com endurecimento, garantindo a unicidade da solução do problema.

Após o início do escoamento, o material passa a apresentar um comportamento elastoplástico. Para a determinação da relação tensão-deformação pós-escoamento, substitui-se o incremento de deformação elástica da eq.(5.15) na eq. (5.16), de forma que o incremento de tensão seja expresso por:

$$
d \sigma_{i j}=C_{i j k l}\left(d \varepsilon_{k 1}-d \varepsilon_{k l}^{p}\right)
$$

Substituindo-se na eq. (5.28) o incremento de deformação plástica dado na eq. (5.27), tem-se:

$$
d \sigma_{i j}=C_{i j k l}\left(d \varepsilon_{k I}-a_{k 1} d \lambda\right)
$$

sendo,

$$
\mathrm{a}_{\mathrm{k} 1}=\frac{\left.\partial \sigma_{\mathrm{k}}, \mathrm{k}\right)}{\partial \sigma_{\mathrm{k} 1}}=\frac{\partial \sigma\left(\sigma_{\mathrm{k} 1}\right)}{\partial \sigma_{\mathrm{k} 1}},
$$

Considerando -se que durante a ocorrência de deformações plásticas o incremento da função ${ }^{F}\left(\sigma_{\mathrm{kI}}, \mathrm{k}\right)$ é nulo, tem-se:

$$
\mathbf{d}=\frac{\partial \sigma}{\partial \sigma_{i j}} d \sigma_{i j}+\frac{\partial F}{\partial k} d k=0
$$

ou, ainda,

$$
a_{i j} d \sigma_{i j}-\frac{\partial Y(k)}{\partial k} d k=0
$$

Substituindo-se, na eq. (5.32), o valor de dk e utilizando-se a hipótese de endurecimento formulada na eq. (5.20), tem-se:

$$
\mathbf{a}_{\mathbf{i j}} d \sigma_{\mathbf{i j}}-\frac{\partial Y(k)}{\partial k} \sigma_{i j} \mathbf{a}_{\mathbf{i j}} \mathbf{d} \lambda=\mathbf{0}
$$

O segundo termo da eq. (5.33), tendo em vista a eq. (5.30), toma a forma : 


$$
\frac{\partial \mathbf{Y}(\mathbf{k})}{\partial \mathbf{k}} \sigma_{i \mathbf{j}} \frac{\partial \mathbf{f}\left(\sigma_{\mathrm{ij}}\right)}{\partial \sigma_{i j}} \mathbf{d} \lambda=\mathbf{0}
$$

Sendo $\mathbf{f}$ uma função homogênea de grau unitário, e aplicando-se o Teorema de Euler para as funções expressas na eq. (5.34), tem-se:

$$
\frac{\partial \mathbf{Y}(\mathbf{k})}{\partial \mathbf{k}} \sigma_{\mathbf{i j}} \mathbf{a}_{\mathbf{i j}} \mathbf{d} \lambda=\frac{\partial \mathbf{Y}(\mathbf{k})}{\partial \mathbf{k}} \mathbf{f}\left(\sigma_{\mathrm{ij}}\right) \mathbf{d} \lambda
$$

Considerando-se $f\left(\sigma_{\mathrm{ij}}\right)$ como uma tensão equivalente $\bar{\sigma}$, para o caso uniaxial e utilizando-se a definição de endurecimento dado na eq. (5.20), pode-se reescrever a eq. (5.35), como:

$$
\frac{\partial \mathbf{Y}(\mathbf{k})}{\partial \mathbf{k}} \mathbf{f}\left(\sigma_{\mathrm{ij}}\right) \mathbf{d} \lambda=\mathbf{H}^{\prime} \mathbf{d} \lambda
$$

sendo que o parâmetro de endurecimento H", expresso na eq. (5.23), relaciona os incrementos de tensão e de deformação plásticas equivalentes.

Substituindo-se a eq. (5.36) e a eq. (5.35) na eq. (5.33), obtém-se:

$$
\mathrm{a}_{\mathrm{ij}} \mathbf{d} \sigma_{\mathrm{ij}}-\mathbb{H} \mathbf{l} d \lambda=\mathbf{0}
$$

O multiplicador plástico $\mathrm{d} \lambda$ pode ser determinado em função do incremento total de deformação, a partir das eq. (5.29) e da eq. (5.37), resultando:

$$
d \lambda=\frac{a_{i j} C_{i j k l}}{a_{i j} d_{i j}+H^{2}} d \varepsilon_{k \mathbf{k l}},
$$

sendo,

$$
d_{i j}=\mathbb{C}_{i j k 1} a_{k 1}
$$

Finalmente, substituindo-se a eq. (5.38) na eq. (5.29), obtém-se a relação incremental entre tensão e deformação, dada por:

$$
d \sigma_{i j}=\left(C_{i j k l}-\frac{d_{i j} a_{m n} C_{m n k l}}{a_{m n} d_{m n}+H^{\prime}}\right) d \varepsilon_{k 1},
$$

Visando a implementação computacional, é conveniente expressar o incremento de tensão em suas componentes elástica e plástica, ou seja:

$$
d \sigma_{i j}=d \sigma_{i j}^{e}-d \sigma_{i j}^{p},
$$


sendo a parcela elástica do incremento dada por:

$$
\mathbf{d} \sigma_{\mathrm{ij}}^{\mathrm{e}}=\mathrm{C}_{\mathrm{ijk1}} \mathbf{d} \varepsilon_{\mathrm{kl} \mathbf{1}}
$$

enquanto a componente plástica resulta em:

$$
d \sigma_{i j}^{p}=\frac{d_{i j} a_{m n}}{a_{m n} d_{m n}+H^{\prime}} d \sigma_{m n}^{e}
$$

Nesta última equação, $\mathbf{d} \boldsymbol{\sigma}_{\mathbf{m n}}^{\mathbf{e}}$ representa o incremento de tensão, devido ao incremento total de deformação, admitindo-se o comportamento elástico, de acordo com a eq. $(5.42)$.

\subsection{CRITÉRIOS DE ESCOAMENTO}

Um critério de escoamento tem por finalidade interpretar as solicitações combinadas, indicando o início das deformações plástica. Assim, a teoria da plasticidade fàz uso das mesmas superficies de escoamento $\mathbb{F}$, definidas na eq. (5.18), interpretando-as, de outro modo, como critérios de plastificação do material.

Dada a grande variedade de materiais empregados (dúcteis, frágeis e outros não tão bem caracterizados), que apresentam comportamento distintos, não é possível ter um único critério.

Neste trabalho tendo em vista que, o solo apresenta resistência à ruptura à tração simples diferente da resistência à compressão, serão considerados apenas os critérios que mais se adaptam a este comportamento.

Um critério de escoamento é independente da orientação do sistema de coordenadas empregado. Assim, é conveniente escreve-los em função do invariante do tensor hidrostático, $\mathbf{I}_{1}$, dado por:

$$
\mathbb{I}_{1}=\sigma_{\mathrm{ii}},
$$

e dos invariantes do tensor anti-esférico, $\mathbb{S}$, dados por:

$$
J_{2}=\frac{1}{2} \mathrm{~S}_{\mathrm{ij}} \mathrm{S}_{\mathrm{ij}}
$$


$\mathrm{e}$,

$$
\mathbf{J}_{3}=\frac{1}{2} \mathrm{~S}_{\mathrm{ij}} \mathrm{S}_{\mathrm{jk}} \mathrm{S}_{\mathrm{ki}}
$$

sendo as componentes de $\mathbf{S}$, dadas por:

$$
\mathrm{S}_{\mathrm{ij}}=\sigma_{\mathrm{ij}}-\frac{1}{3} \delta_{\mathrm{ij}} \sigma_{\mathrm{kk}}
$$

Uma representação alternativa para estes invariantes, consiste em substituir o invariante $\mathbb{J}_{\mathbf{3}}$, pelo ângulo $\theta$, originalmente introduzido por LODE (1926), e dado por:

$$
\theta=\frac{1}{3} \operatorname{arcsen}\left(-\frac{3 \sqrt{3} J_{3}}{2} \frac{J_{2}^{3 / 2}}{-}\right.
$$

com o ângulo $\theta$ variando no intervalo $[-\pi / 6, \pi / 6]$.

Com os três invariantes, $\mathbb{I}_{1}, \mathbb{J}_{2}$ e $\mathbf{J}_{3}$ ou $\theta$, é possivel definir várias superficies de escoamento as quais representam os diversos critérios de plastificação. Ao longo do tempo vários critérios foram estabelecidos, sendo os mais conhecidos os critérios de Von Mises, Tresca, Mohr-Coulomb e Drucker-Prager. Os dois primeiros critérios apresentam resultados mais satisfatórios para materiais dúcteis, enquanto os demais são mais indicados para materiais frágeis.

No estudo da plasticidade em solos e rochas, geralmente são utilizados os critérios de plastificação de Mohr-Coulomb e Drucker-Prager.

\subsubsection{CRITÉRIO DE MOHR-COULOMR}

A superficie de escoamento definida pelo critério de Mohr-Coulomb é uma generalização da teoria de atrito de Coulomb, apresentada em 1773 e demonstrada inicialmente por Mohr em 1882. Neste critério, a plastificação do material em um determinado ponto ocorre quando a tensão de cisalhamento alcança um valor crítico, dado por:

$$
\tau=\mathbb{C}-\sigma \mathrm{g} \dot{\varphi},
$$


sendo que, $\tau$ é a tensão de cisalhamento, $\sigma$ é a tensão normal no plano de ruptura, c é a coesão e $\phi$ o ângulo de atrito interno do material.

Da observação da Fig. 5.5, constata-se que:

$$
\sigma=\frac{\sigma_{1}+\sigma_{3}}{2}
$$

e,

$$
\tau \cos \phi=\frac{\sigma_{1}-\sigma_{3}}{2}
$$

Portanto, a eq. (5.49), pode ser escrita na forma:

$$
\frac{\left(\sigma_{1}-\sigma_{3}\right)}{2}+\frac{\left(\sigma_{1}+\sigma_{3}\right)}{2} \operatorname{sen} \phi=\cos \phi
$$

ou, rearranjando os termos, tem-se:

$$
\left(\sigma_{1}-\sigma_{3}\right)=2 \cos \phi-\left(\sigma_{1}+\sigma_{3}\right) \operatorname{sen} \phi \text {, para } \sigma_{1} \geq \sigma_{2} \geq \sigma_{3}
$$

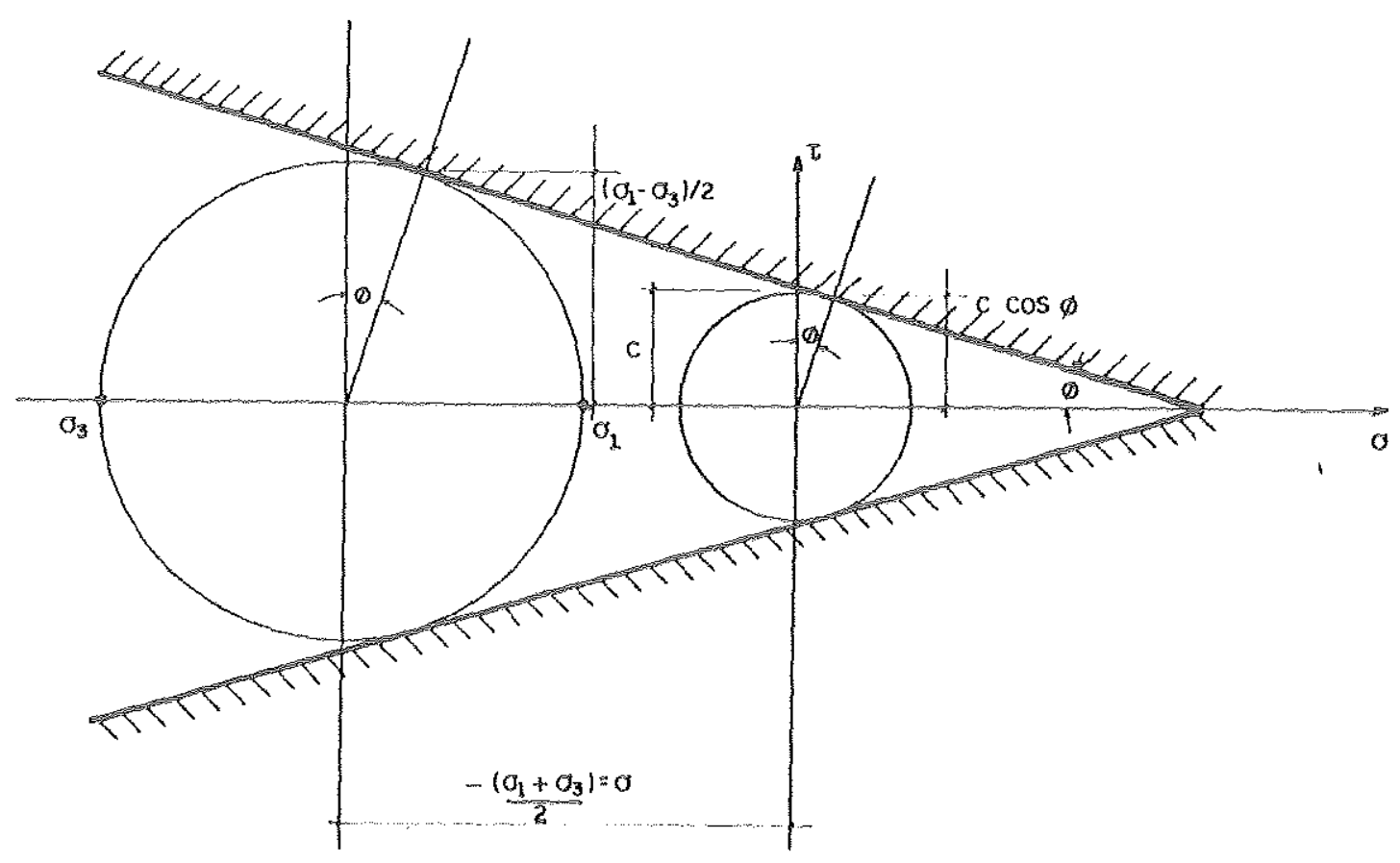

FIGURA 5.5 - Representação do critério de Mohr-Coulomb.

É possível escrever o critério de escoamento em função dos invariantes $\mathbb{1}_{1}, \mathbb{J}_{2}$ e $\mathbb{J}_{3}$ ou $\theta$ e dos parâmetros c e $\phi$, como: 


$$
F=\frac{1}{3} I_{1} \operatorname{sen} \phi+\sqrt{J_{2}}\left(\cos \theta-\frac{1}{\sqrt{3}} \operatorname{sen} \theta \operatorname{sen} \phi\right)-\cos \phi=0
$$

\subsubsection{CRITÉRIO DE DRUCKER-PRAGER}

O critério proposto por DRUCKER\&PRAGER (1952) é uma modificação do critério de escoamento de Von Mises, acrescentando-se uma parcela relativa ao primeiro invariante, $\mathbf{I}_{1}$, obtendo-se:

$$
\mathbb{F}=\alpha \mathbb{I}_{1}+\sqrt{\mathbb{J}_{2}}-\mathbf{k}=\mathbf{0}
$$

onde $\alpha$ e k são constantes positivas, relacionadas aos parâmetros c e $\phi$, e com valores particulares para cada caso de aproximação desejada.

Esta superfície, representada no espaço das tensões, tem a forma de um cone, enquanto que a superficie determinada pelo critério de Mohr-Coulomb tem a forma de uma pirâmide hexagonal irregular (função do valor do ângulo $\phi$ ). As duas superficies têm vértices coincidentes no ponto $\sigma_{1}=\sigma_{2}=\sigma_{3}=\operatorname{cotg} \phi$ ( Fig. 5.6).

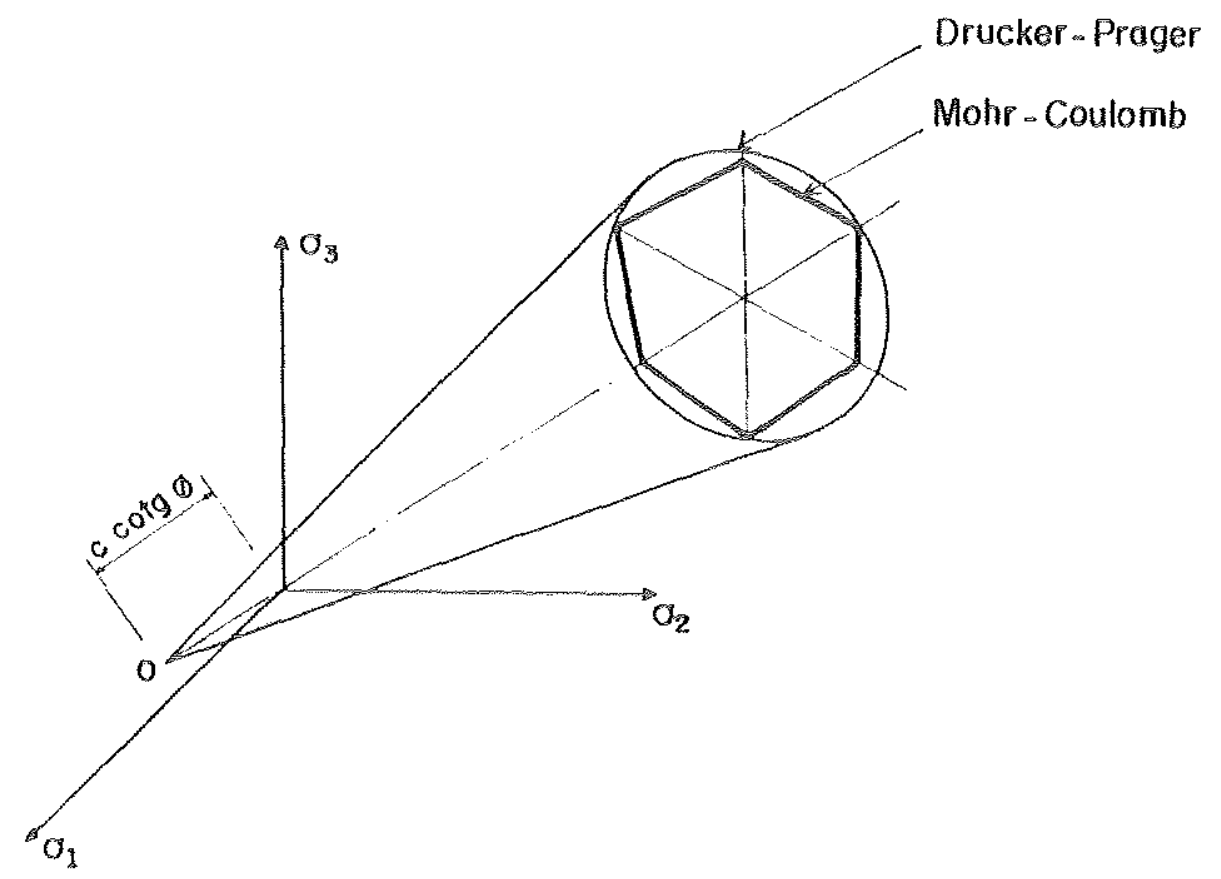

FIGURA 5.6 - Representação geométrica das superfícies de escoamento de MohrCoulomb e Drucker-Prager no espaço das tensões principais. 
Para a obtenção dos valores de $\alpha$ e $\mathbf{k}$, por exemplo, pode-se admitir a hipótese que o cone de Drucker-Prager circunscreve a pirâmide de Mohr-Coulomb. Assim:

$$
\alpha=\frac{2 \operatorname{sen} \phi}{\sqrt{3}(3-\operatorname{sen} \phi)} \quad \mathrm{k}=\frac{6 \cos \phi}{\sqrt{3}(3-\operatorname{sen} \phi)}
$$

Por outro lado, se o cone estiver inscrito à pirâmide, tem-se:

$$
\alpha=\frac{2 \operatorname{sen} \phi}{\sqrt{3}(3+\operatorname{sen} \phi)} \quad \text { e } \quad k=\frac{6 \cos \phi}{\sqrt{3}(3+\operatorname{sen} \phi)}
$$

A Fig. 5.7, ilustra a comparação no plano $\sigma_{1}-\sigma_{2}$ entre os cones inscritos e circunscrito à pirâmide representativa do critério de Mohr-Coulomb.

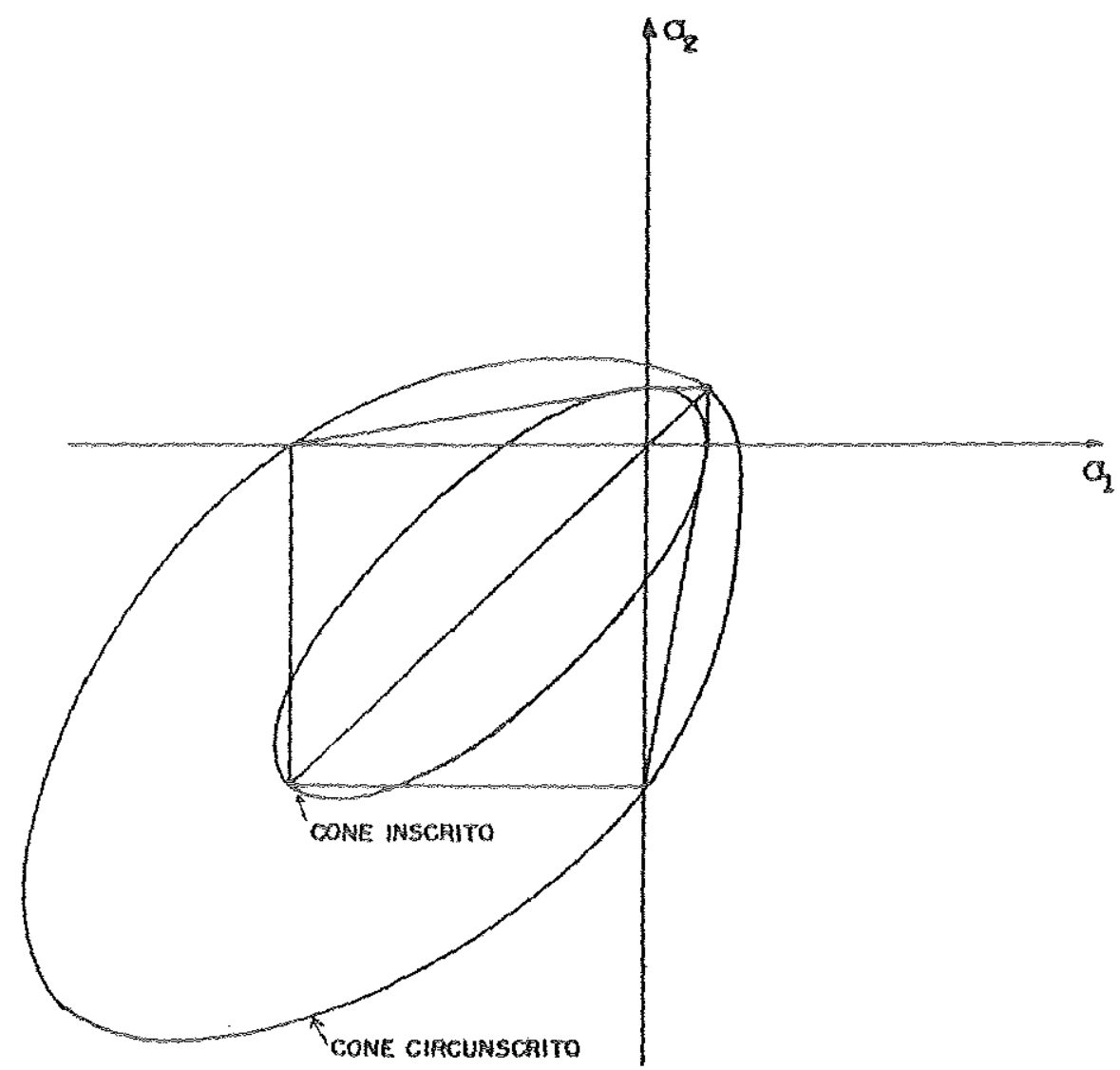

FIGURA 5.7 - Representação geométrica dos critérios de Mohr-Coulomb e Drucker -Prager, no plano de tensôes $\sigma_{1}-\sigma_{2}$.

Outros valores para as constantes $\alpha$ e $k$ podem ser definidos na tentativa de melhor representar o comportamento elastoplástico, tanto de solos como de rochas. 


\subsection{FORMULAÇÃO MATRICIAL DAS RELAÇÕES CONSTITUTIVAS}

Visando a implementação computacional, é conveniente que algumas expressões anteriormente apresentadas, sejam escritas na forma matricial. Inicialmente, a eq. (5.18) que representa o critério de escoamento é reescrita na sua forma original,

$$
\mathbf{F}\left(\sigma_{\mathrm{ij}}, \mathbf{k}\right)=\mathbf{f}\left(\sigma_{\mathrm{ij}}\right)-\mathbf{Y}(\mathbf{k})=\mathbf{0},
$$

que por diferenciação, obtém-se:

$$
\mathbf{d} \mathbf{F}=\frac{\partial \mathbf{F}}{\partial \sigma} \mathbf{d} \sigma+\frac{\partial \mathbf{F}}{\partial \mathbf{k}} \mathbf{d} \mathbf{k}=\mathbf{0}
$$

ou, na forma matricial,

$$
\stackrel{a}{ }^{\mathrm{T}} d \sigma-H_{\sim} d \lambda=0
$$

sendo a , o vetor de fluxo, dado por:

$$
\mathfrak{a}^{\mathbf{T}}=\frac{\partial \mathbb{F}}{\partial \sigma}=\left\{\frac{\partial \mathbb{F}}{\partial \sigma_{11}}, \frac{\partial \mathbb{F}}{\partial \sigma_{22}}, \frac{\partial \mathbb{F}}{\partial \sigma_{33}}, \frac{\partial \mathbb{F}}{\partial \sigma_{23}}, \frac{\partial \mathbb{F}}{\partial \sigma_{31}}, \frac{\partial \mathbb{F}}{\partial \sigma_{12}}\right\}
$$

A eq. (5.40), que relaciona os incrementos de tensão e deformação após o escoamento, fica:

$$
\mathbb{\sim} \underset{\sim}{\sigma}=\underset{\sim \mathrm{ep}}{\mathbb{D}} \mathbb{\sim} \varepsilon
$$

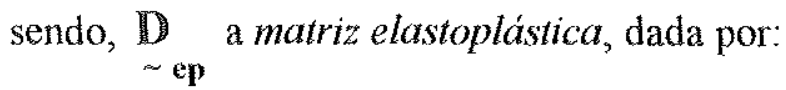

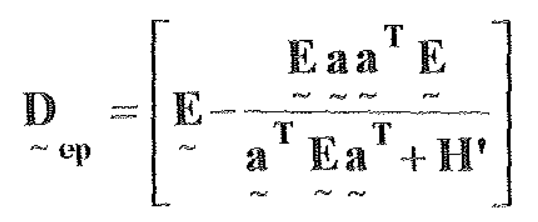

onde $\mathbb{E}$ é a matriz de constantes elásticas do material.

Portanto, para a deteminação da matriz elastoplástica, indicada na eq. (5.62), basta que se conheça o vetor de fluxo para cada critério de escoamento, que como já 
descrito, pode ser denotado em função dos invariantes. De acordo com NAYAK \& ZIENKIEWICZ (1972), o vetor a pode ser expresso de forma geral, como:

$$
\mathbf{a}=\mathbf{C}_{1} \underset{\sim 1}{\mathbf{a}}+\mathbf{C}_{2} \underset{\sim 2}{\mathbf{a}}+\mathbf{C}_{3} \underset{\sim 3}{\mathbf{a}}
$$

sendo,

$$
\begin{aligned}
& \underset{\sim 1}{\mathbf{a}^{\mathrm{T}}}=\frac{\partial \mathbb{I}_{1}}{\partial \sigma} \\
& \underset{\sim}{\mathbf{a}^{\mathrm{T}}}=\frac{\partial\left(\mathbb{J}_{2}\right)^{1 / 2}}{\partial \sigma} \\
& \underset{\sim}{\mathbf{a}^{\mathbf{T}}}=\frac{\partial \mathbb{J}_{3}}{\partial \sigma}
\end{aligned}
$$

Assim, somente as constantes $\mathbf{C}_{1}, \mathbb{C}_{2}$ e $\mathbb{C}_{3}$ são necessárias para definir o critério de escoamento. No caso do critério de Mohr-Coulomb, estas constantes valem:

$$
\begin{aligned}
& C_{1}=\frac{1}{3} \operatorname{sen} \phi \\
& C_{2}=\cos \theta[(1+\operatorname{tg} \theta \operatorname{tg} 3 \theta)+\operatorname{sen} \phi(\operatorname{tg} 3 \theta-\operatorname{tg} \theta) / \sqrt{3}] \\
& C_{3}=\frac{\sqrt{3} \operatorname{sen} \theta+\cos \theta \operatorname{sen} \phi}{2 \cdot l_{2} \cos 3 \theta}
\end{aligned}
$$

Para o critério de Drucker-Prager as constantes são dadas por:

$$
\begin{aligned}
& C_{1}=\alpha \\
& C_{2}=1,0 \\
& C_{3}=0,0
\end{aligned}
$$

A superficie de escoamento de Mohr-Coulomb, definida geometricamente no espaço das tensões como uma pirâmide, apresenta descontinuidade nas derivadas em alguns pontos (arestas). Por exemplo, para $\theta= \pm 30^{\circ}$ a direção das deformações plástica são indeterminadas. Uma aproximação consiste em substituir a superficie de 
Mohr-Coulomb, pela superfície de escoamento de Drucker-Prager, quando $|\theta| \geq \mathbf{2 9}^{\circ}$, ajustada de modo a estar inscrita ou circunscrita à pirâmide de Mohr-Coulomb.

\subsection{UM MODELO SIMPLES PARA A ANÁLISE ELASTOPLÁSTICA DE SOLOS NA INTERFACE COM ESTACAS}

Ao formular o presente modelo matemático para a análise da interação solofundação em estacas, no espaço tridimensional, vários são os fatores que contribuem para um comportamento não-linear, podendo-se citar: a não linearidade inerente ao comportamento do próprio solo, além de efeitos que se revelam na interface estaca-solo, como o escorregamento ou deslizamento, que se caracteriza pela não compatibilidade de deslocamentos entre o solo e a estaca, e a separação que pode ocorrer em razão do tipo de carregamento a que a estaca está submetida.

O escorregamento tem início, simultaneamente como escoamento do solo, tal que, na interface solo-estaca estabelece-se um estado de tensão, que corresponde a um ponto sobre a superficie de escoamento do solo. A partir daí, os deslocamentos dão-se de forma distinta entre o solo e a estaca, sendo que na estaca, os deslocamentos são muito superiores aos que ocorrem no solo.

A aplicação de um carregamento lateral à estaca, pode provocar o aparecimento de tensões de tração no solo, que quando superam a tensão de ruptura à tração, levam a separação entre a estaca e o solo. Este efeito pode se considerado mediante uma formulação do MEC para fratura mecânica (VENTURINI ,1994).

Estes dois efeitos não serão tratados neste trabalho, embora seja possível resolvelos, também via tratamento numérico, dando continuidade à presente linha de pesquisa.

Neste modelo, como apresentado no Capínulo 4, a solução é obtida a partir de uma combinação MEC-MEF. Assim, as equações vistas no item anterior, após serem convenientemente modificadas, podem ser utilizadas na análise do comportamento nãolinear do solo. 
Para um incremento de carga genérico, d F , e admitindo-se o comportamento elástico, o incremento de deslocamentos correspondente, $\underset{\sim}{\mathbf{d}}{ }^{\mathbf{e}}$, é obtido, a partir da eq. (5.26), como:

$$
\mathbf{d} \mathbf{U}^{\mathbf{e}}=\underset{\sim}{\mathbf{K}^{-1} \mathbf{d} \mathbf{F}}
$$

sendo, $\mathbf{K}$ a matriz de rigidez equivalente do sistema solo-estaca.

Os incrementos de deslocamentos determinam na interface solo-estaca o aparecimento de incrementos de componentes de forças nodais, que podem ser calculados a partir da eq. (5.17) e expressa como:

$$
\mathbf{d} \mathbf{Q}^{\mathrm{e}}=\mathbf{S}^{\boldsymbol{P}^{-1}} \mathbf{d} \mathrm{U}^{\mathrm{e}}-\mathbf{d} \mathbf{Q}^{\mathbf{0}}
$$

sendo que, $\mathbf{d} \mathbf{Q}^{\mathrm{e}}$, representa os incrementos de componentes de forças de interação e $\mathbf{d Q}^{\mathbf{0}}$, os incrementos de forças conhecidas atuando sobre o contorno ou sobre a superficie do meio, nas três direções coordenadas.

As componentes de forças nodais determinam no solo em sua interface com a estaca o seguinte estado de tensão. No plano $\mathbb{x}_{1}-\mathrm{X}_{2}$, as componentes de forças são somadas vetorialmente, obtendo-se uma resultante. A tensão normal neste plano é obtida pela divisão desta resultante pelo diâmetro da estaca. A componente de força na direção $\mathbb{x}_{3}$, por sua vez, ao ser dividida pelo perímetro da estaca, detemina uma tensäo de cisalhamento em um plano vertical. Uma outra componente de tensão normal, na direção vertical, pode ser inclúda ao modelo, como conseqüência do peso próprio do solo. Assim, fica definido um estado plano de tensões, dado por:

$$
\sigma^{\mathrm{e}}=\left\{\begin{array}{l}
\sigma_{11}^{\mathrm{e}} \\
\sigma_{22}^{\mathrm{e}} \\
\sigma_{12}^{\mathrm{e}}
\end{array}\right\}
$$

Diferentemente de outros modelos de comportamento não-linear onde se estabelecem relações entre tensões e deformações, neste caso as tensões estão relacionadas diretamente aos deslocamentos, como mostram as eq. (5.67) e (5.68). 
Após o escoamento, as relações incrementais apresentada na eq. (5.40), podem ser escritas na forma:

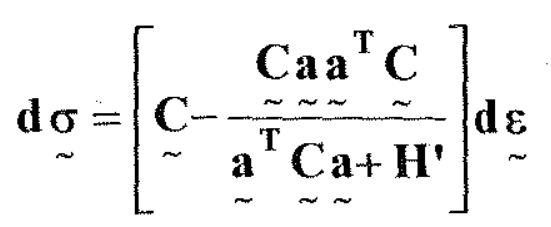

sendo que $\mathbb{C}$ a matriz de constantes elásticas dada por:

$$
\widetilde{C}=\frac{\mathbb{E}}{1-v^{2}}\left[\begin{array}{ccc}
1 & v & 0 \\
v & 1 & 0 \\
0 & 0 & \frac{1-v}{2}
\end{array}\right]
$$

onde, $\mathbb{E}$ e $v$ são o módulo de deformação e o coeficiente de Poisson do solo, respectivamente. O vetor de fluxo, a, por sua vez, para o estado plano de tensão é dado por:

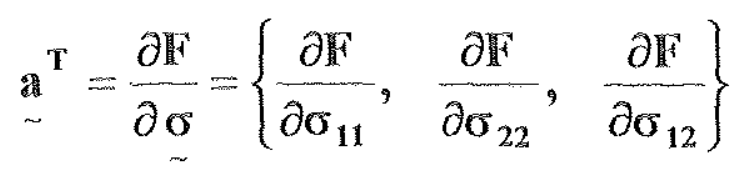

Tendo em vista as eq. (5.38) e (5.39), pode-se escrever a eq. (5.70) como:

$$
d \sigma=d \sigma^{e}-d \lambda d
$$

sendo que:

$$
\mathrm{d} \lambda=\frac{\mathrm{a}_{\sim}^{\mathrm{T}} \mathrm{Ca}+\mathrm{H}^{\mathrm{C}}}{\sim} \mathrm{\sim}
$$

$\mathrm{e}$

$$
\boldsymbol{d}=\mathbb{C}
$$

A partir da eq. (5.75), o vetor $d$, pode ser explicitado na forma: 


$$
\underset{\sim}{\mathbf{d}}=\left\{\begin{array}{l}
\mathbf{d}_{1} \\
\mathbf{d}_{2} \\
\mathbf{d}_{3}
\end{array}\right\}=\left\{\begin{array}{c}
\frac{\mathbf{E}}{1+v} \mathbf{a}_{1}+\mathbf{M}_{1} \\
\frac{\mathbf{E}}{1+v} \mathbf{a}_{2}+\mathbf{M}_{1} \\
\mathbf{G} \mathbf{a}_{3}
\end{array}\right\}
$$

na qual, G é o módulo de deformação transversal do solo, $\mathbf{a}_{1}, \mathbf{a}_{2}$ e $\mathbf{a}_{3}$ são as componentes do vetor a e $\mathbf{M}_{\mathbf{1}}$, vale:

$$
\mathbf{M}_{1}=\frac{\mathbf{E v}\left(\mathbf{a}_{1}+\mathbf{a}_{2}\right)}{1-v^{2}}
$$

Pode-se observar que a parcela $d \lambda d$ da eq. (5.73) representa a componente plástica do incremento de tensões, que deverá ser reaplicada ao sistema como incremento de carga. Assim, as componentes plásticas de tensão são transformadas em incrementos de componente de forças nodais, segundo um procedimento inverso ao efetuado anteriormente ao se converter componentes de forças nodais em tensões. Determinados os incrementos de componentes de forças nodais, estes são transformados em incrementos de forças nodais equivalentes, utilizando-se a matriz $\mathbb{T}$, como indicado na eq. (5.21), ou seja:

$$
\mathrm{d} \mathbf{\mathrm { C }}=\mathbf{T} \mathrm{T} \mathbf{Q}
$$

Assim, a resultante dos incrementos de forças nodais equivalentes, obtida a partir da eq. (5.78), será reaplicada ao sistema como um incremento de carga.

\subsection{PROCLDIMENTO NUMERICO PARA A SOLUCÃO DO PROBLLMA NÃO-LINEAR}

Utilizando-se o equacionamento descrito nos itens anteriores, é apresentado a seguir um procedimento numérico para a solução de problemas elastoplásticos, baseado 
no processo das tensões iniciais proposto por ZIENKIEWICS et al. (1969), para o método dos elementos finitos.

Tendo em vista a natureza incremental da relação tensão-deformação para o comportamento pós-escoamento, dada pela eq. (5.61), o processo numérico para a solução do problema requer que o carregamento aplicado à estaca seja feito de forma incremental. Utiliza-se, então, um algoritmo incremental-iterativo, sem atualização das matrizes envolvidas, conhecido como método da rigidez inicial, análogo ao apresentado por OWENS \& HINTON (1980). Nesta referência, está detalha a abordagem numérica que é empregada no presente trabalho, apresentando-se a seguir apenas os aspectos gerais do algoritmo.

Para um incremento de carga genérico e admitindo-se o comportamento elástico, pode-se determinar o incremento de deslocamentos aplicando-se a eq. (5.67), e as componentes de forças nodais, através da eq. (5.68), que conforme mostrado no item anterior, resultam em incremento de tensões no solo, nos pontos da interface estaca.solo. Este incremento de tensões é acumulado ao estado de tensões atual. Se algum ponto atinge o escoamento, o incremento de tensões reais, que verifica a condição de escoamento, deve ser calculado e o excesso de tensões, que consiste no incremento de tensóes plásticas, $\Delta \sigma_{\sim}^{p}$, deve ser reaplicado ao sistema na forma de incremento de cargas.

Um procedimento que pode ser utilizado é o de se obter a solução elástica para um carregamento total previsto e definir os incrementos de cargas a partir de uma fração $\beta_{\mathbf{i}}$ do carregamento. Assim, para um incremento de carga genérica $\mathbf{i}$, o incremento nas tensões elásticas, vale:

$$
\underset{\sim \mathbf{i}}{\Delta \sigma^{\mathrm{e}}}=\beta_{\mathrm{i}} \stackrel{\sim}{\sim}^{\mathrm{c}}
$$

A medida que o carregamento vai sendo aplicado, os fatores $\beta_{\mathbf{i}}$ vão sendo acumulados.

A seqüência básica utilizada para cada incremento de carga pode ser resumidamente descrita como:

(a) Calcula-se o incremento elástico das tensões, $\Delta \underset{\sim i}{\Delta}$, através da eq. (5.79), para a primeira iteração, a partir do incremento de carga. Para as demais iterações, 
$\underset{\sim i}{\sigma}{ }_{\sim i}^{\mathrm{e}}$ é calculado a partir do excesso de tensões, que corresponde ao incremento plástico, $\underset{\sim i-1}{\Delta \sigma^{p}}$, que transformado em componentes de cargas nodais, é aplicado como incremento do carregamento, de acordo com a eq. (5.78):

$$
\underset{\sim \mathbf{i}}{\Delta \mathbf{F}^{\mathrm{e}}}=\underset{\sim}{\mathbf{T}} \Delta \mathbf{Q}_{\sim \mathbf{i}-\mathbf{1}}^{\mathbf{p}}
$$

(b) Em cada ponto do solo, calcula-se o estado de tensões suponde-se o

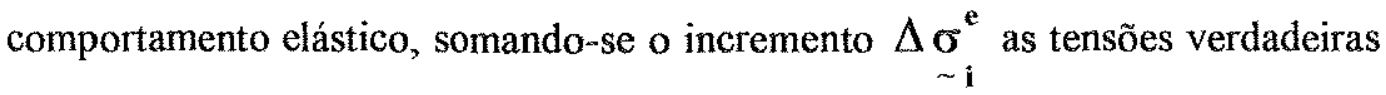
da iteração anterior, ou seja:

$$
\underset{\sim \mathbf{i}}{\sigma^{e}}=\underset{\sim \mathbf{i}-\mathbf{1}}{\sigma}+\underset{\sim \mathbf{i}}{\sigma}
$$

(c) O próximo passo consiste em se verificar a condição de escoamento. Ou seja, se $\mathfrak{f}\left(\begin{array}{c}\sigma_{\sim \mathrm{i}}^{\mathrm{e}} \\ \sim \mathrm{i}\end{array}\right)<\sigma_{\mathrm{y}}(\mathrm{k})$; o ponto ainda está em regime elástico e o valor de $\underset{\sim \mathrm{i}}{\sigma^{\mathrm{e}}}$ está correto. Na desigualdade acima, $\sigma_{y}\left(k_{k}\right)$ é a tensão de escoamento atualizada no final da iteração anterior, sendo dada por:

$\sigma_{\mathrm{y}}(\mathrm{k})=\sigma_{0}+\mathrm{T}^{\mathrm{I}} \bar{\varepsilon}_{\mathrm{i}-1}^{\mathrm{p}}$

sendo que, $\bar{\varepsilon}_{\mathbf{i}-1}^{p}$ é a deformação plástica acumulada até a (i-1)-ésima iteração.

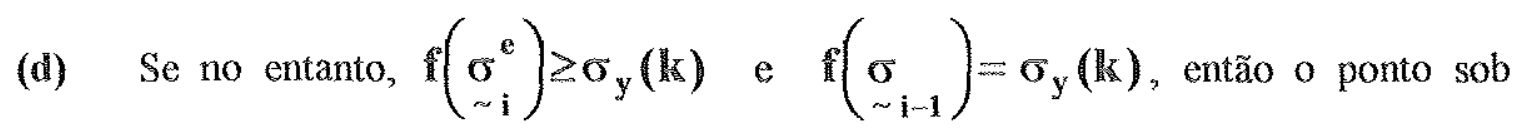
consideração já havia escoado na iteração anterior e as tensões ainda estão sendo aumentadas. Portanto, todo o excesso de tensões deve ser reduzido, de forma que o ponto permaneça na superficie de escoamento. Neste caso, o incremento de tensões deve ser calculado através da eq. (5.73), isto é:

$$
\Delta \sigma=\Delta \sigma_{\sim}^{e}-d \lambda
$$

e o estado de tensões nesta iteração, que satisfaz o critério de escoamento, será:

$$
\underset{\sim \mathbf{i}}{\sigma}=\underset{\sim \mathbf{i}-1}{\sigma}+\Delta \sigma_{\sim}^{\mathrm{e}}-\boldsymbol{u} \underset{\sim}{\mathbf{l}}
$$


$\mathrm{O}$ último termo da eq. $(5,84)$, corresponde à componente plástica do incremento de tensões na iteração em curso, ou seja:

$$
\underset{\sim \mathbf{i}}{\Delta \sigma^{p}}=\mathbf{d} \lambda \mathbf{d}
$$

Este incremento de tensão plástica é acumulado com $\underset{\sim}{\sigma^{p}}$ e é aplicado na próxima iteração como incremento de carga.

O cálculo do incremento da deformação plástica efetiva é feito a partir do conceito do trabalho plástico, dado na eq. (5.20), ou seja:

$$
d w_{p}=\underset{\sim i}{\sigma} d \varepsilon_{\sim i}^{p}=d \lambda \underset{\sim}{\varepsilon^{T}} \underset{\sim i}{\sigma}
$$

ou, reescrevendo-se em termos de tensão efetiva e deformação efetiva, tem-se:

$$
\bar{\sigma} \mathbf{d} \bar{\varepsilon}_{\mathbf{i}}^{\mathrm{p}}=\mathbf{d} \lambda \underset{\sim}{\mathrm{T}} \underset{\sim \mathbf{i}}{\mathrm{T}}
$$

Portanto, a deformação plástica efetiva ou equivalente, será:

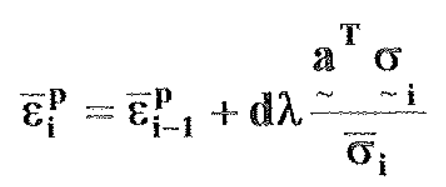

onde, a tensão efetiva ou equivalente, $\bar{\sigma}_{i}$, é igual ao valor de $\left(\begin{array}{l}\sigma_{\sim} \\ \sim_{1}\end{array}\right)$.

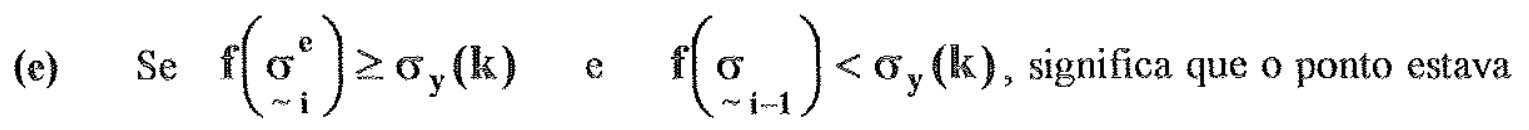
em regime elástico no inicio e houve escoamento durante a iteração corrente. Determinam-se, então os valores de tensão que satisfazem o critério de escoamento. A parcela acima destes valores deve ser reduzida à superficie de escoamento, de modo análogo ao apresentado no item anterior.

(f) Verifica-se a convergência. Caso não tenha ocorrido retorna-se ao passo (a) para uma nova iteração. Quando ocorre a convergência, um novo incremento de carga é aplicado, repetindo-se todos os passos descritos, até que o carregamento total seja aplicado. Considera-se, neste trabalho, que a convergência é verificada quando o excesso de tensões efetivas fica abaixo de uma certa percentagem da tensão de plastificação da iteração atual. 


\subsection{APLICAÇÕES NUMÉRICAS}

Apresentam-se, a seguir, alguns exemplos simples para análise do comportamento não-linear do solo, na interface com a estaca. O modelo desenvolvido é comparado, com ensaios realizados, ou com valores obtidos a partir de outros métodos.

\subsubsection{Exemplo 1 - Ensaio de Whitaker e Cooke}

Este ensaio está apresentado em POULOS \& DAVIS (1980). Uma estaca vertical em concreto, com comprimento de $12,2 \mathrm{~m}$ e diâmetro igual a $0,61 \mathrm{~m}$, é submetida a uma carga vertical. O solo é caracterizado com argiloso, com módulo de deformação de $8.10^{4} \mathrm{kN} / \mathrm{m}^{2}$ e coeficiente de Poisson igual a 0,5 e uma resistência ao atrito lateral igual a $37,9 \mathrm{kN} / \mathrm{m}^{2}$. O concreto da estaca apresenta módulo de deformação igual a $2,067 \cdot 10^{7} \mathrm{kN} / \mathrm{m}^{2}$.

A estaca foi discretizada em sete ponto nodais e o problema foi processado com 22 incrementos de carga. Considerou-se o solo com comportamento elastoplástico perfeito. A carga para a qual ocorre o escoamento do solo nos sete pontos nodais é de $886 \mathrm{kN}$. Para qualquer incremento de carga aplicado à estaca após alcançado este valor, não se obtém mais a convergência, como era de se esperar. Para a carga de $886 \mathrm{kN}$, o valor do deslocamento vertical medido no ensaio foi de $4,45 \mathrm{~mm}$. O presente exemplo foi processado para três valores de módulo de deformação do solo iguais a 80000 $\mathrm{kN} / \mathrm{m}^{2}, 110000 \mathrm{kN} / \mathrm{m}^{2}$ e $200000 \mathrm{kN} / \mathrm{m}^{2}$. Para este valores do módulo de deformação do solo o deslocamento observado para a carga última da estaca é de $5,37 \mathrm{~mm}, 4,39 \mathrm{~mm}$ e $3,29 \mathrm{~mm}$, respectivamente. 


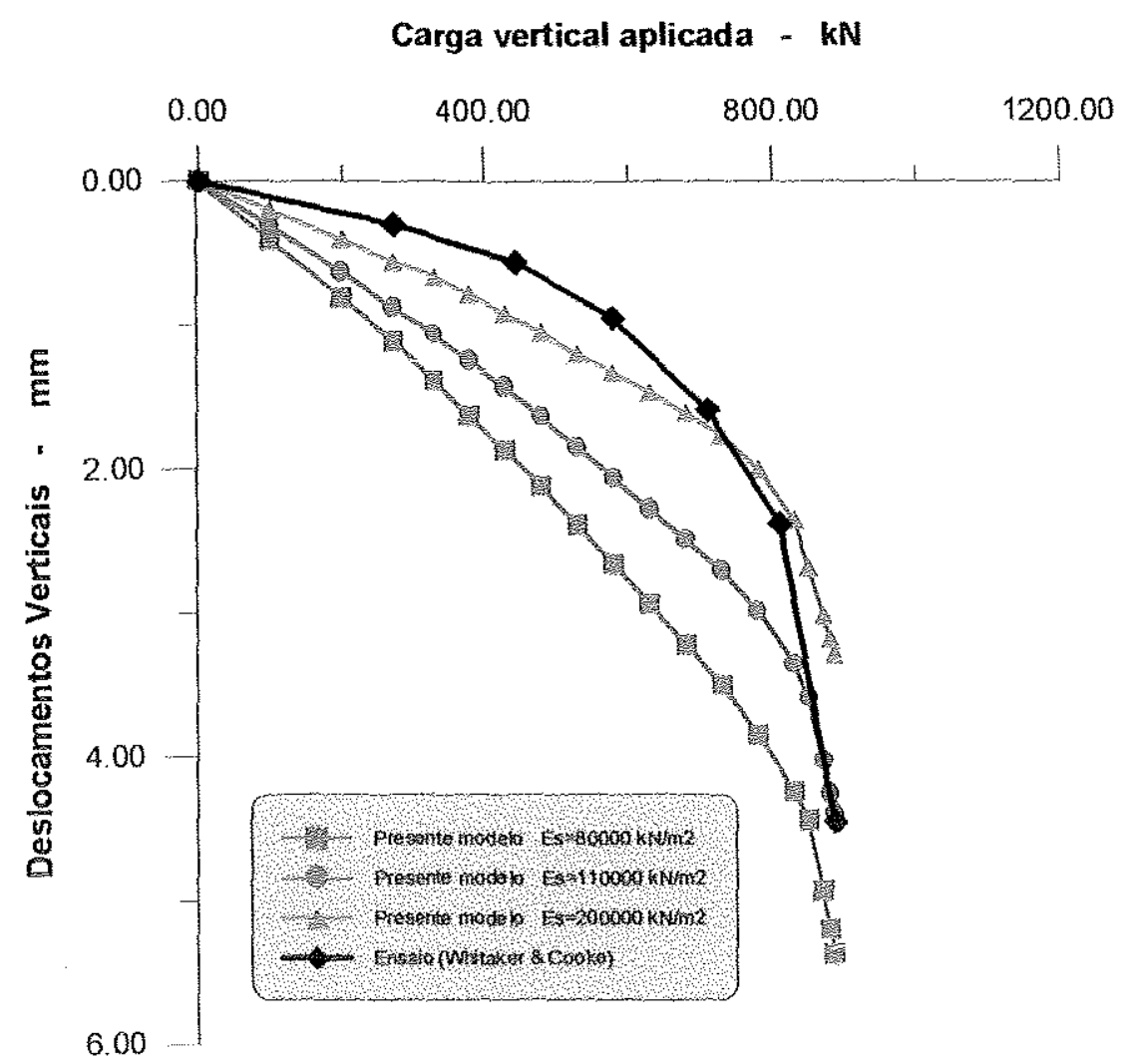

FIGURA 5.8 - Curva carga-deslocamento no topo da estaca.

Os resultados do ensaio e das análises estão apresentados na Fig. 5.8. Este exemplo tem a finalidade básica de aferir o modelo e verificar o comportamento das curvas carga-recalque, considerando além dos fatores intrínseco ao modelo a variação do módulo de deformação do solo, nesse comportamento. Assim, tendo em vista o solo ser um material com características e propriedades que não permitem classifica-lo como homogêneo e isótropo, hipóteses assumidas ao desenvolver o modelo, entende-se que os resultados obtidos são bastante satisfatórios.

\subsubsection{Exemplo 2- Estaca vertical com carga vertical}

Neste exemplo, uma estaca de concreto protendido, pré-moldada, com comprimento de $8,5 \mathrm{~m}$ e seção transversal quadrada de lado $0,4 \mathrm{~m}$ é considerada. Segundo CHEUNG ế al. (1991), para efeito de modelagem, admitiu-se um diâmetro de $0,451 \mathrm{~m}$, dando a mesma área de seção transversal. A estaca foi cravada em um solo siltoso com módulo de deformação igual a $5000 \mathrm{kN} / \mathrm{m}^{2}$, coeficiente de Poisson de 0,35 
e resistência de atrito lateral de $44,84 \mathrm{kN} / \mathrm{m}^{2}$. O solo foi admitido tendo comportamento elastoplástico perfeito. A estaca é vertical, sob carga vertical, aplicada em seu topo, tendo módulo de deformação igual a $2,068 \cdot 10^{7} \mathrm{kN} / \mathrm{m}^{2}$. Neste exemplo também a estaca foi discretizada em 7 nós, e foram aplicados 23 incrementos de carga, sendo que no último não se obteve a convergência. Na Fig. 5.9, indica-se, em curva cargadeslocamento, os resultados da prova de carga, a solução obtida com a utilização do modelo de Duncan-Chang, usado para análise não-linear pelo método dos elementos finitos, o modelo Cambridge modificado, o modelo Yin e os resultados obtidos pelo modelo proposto considerando-se dois valores para o módulo de deformação do solo $\left(5000 \mathrm{kN} / \mathrm{m}^{2}\right.$ e $\left.7000 \mathrm{kN} / \mathrm{m}^{2}\right)$.

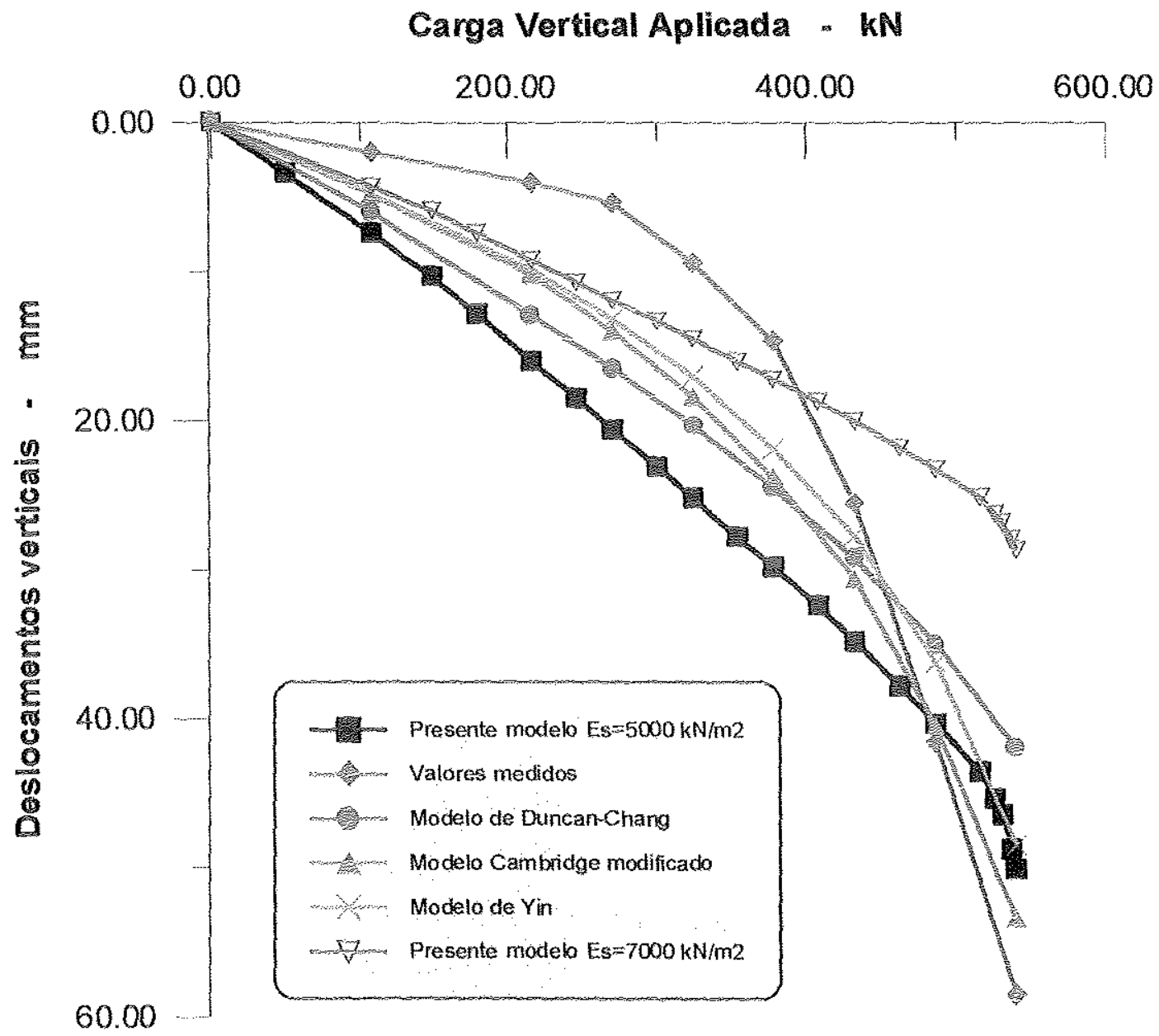

FIGURA 5.9 - Curva carga-deslocamento no topo da estaca.

Para a carga de $540 \mathrm{kN}$, correspondente a carga de ruptura, o deslocamento determinado em ensaio é de $58,4 \mathrm{~mm}$, enquanto os calculados a partir do modelo de 
Duncan-Chang é de $41,9 \mathrm{~mm}$, modelo Cambridge modificado $53,3 \mathrm{~mm}$, modelo de Yin $48,4 \mathrm{~mm}$, enquanto para o modelo proposto é de $50 \mathrm{~mm}$ e $28,6 \mathrm{~mm}$, considerando-se o módulo de deformação do solo igual a $5000 \mathrm{kN} / \mathrm{m}^{2}$ e $7000 \mathrm{kN} / \mathrm{m}^{2}$, respectivamente. Pode-se constatar uma boa concordância de deslocamentos para a carga de colapso da estaca entre o ensaio e o modelo ora proposto (para $\mathrm{E}_{\mathrm{s}}=5000 \mathrm{kN} / \mathrm{m}^{2}$ ). No entanto, como pode-se observar à Fig. 5.9, os deslocamentos calculados, para pequenas cargas aplicadas são maiores do que aqueles medidos no ensaio. Assumindo-se para o módulo de deformação do solo um valor igual a $7000 \mathrm{kN} / \mathrm{m}^{2}$, essas diferenças, tendem a diminuir para pequenas cargas aplicadas, mas, para a carga de ruptura os deslocamentos calculados, na presente análise, tornam-se inferiores aos obtidos no ensaio. Isto demonstra que o solo sofreu um pré-adensamento quando da instalação da estaca. Este efeito de pré-adensamento pode vir a ser considerado no modelo, por exemplo, a partir da adoção de um diagrama tensão-deformação trilinear, tornando o modelo mais realista. 


\section{CONCLUSÕES}

Neste trabalho foi desenvolvido uma combinação entre o método dos elementos de contorno (MEC) e o método dos elementos finitos (MEF). Cada um dos métodos se mostra mais conveniente para tratar determinados tipos de domínios. $\mathrm{O}$ método dos elementos de contorno é mais adequado na análise de problemas que envolvam sólidos tridimensionais de domínio infinito ou semi-infinito. A adoção da solução fundamental de Mindlin para esta formulação é indicada pois elimina a necessidade de discretização da superfície livre, reduzindo o número de incógnitas do problema e, consequentemente, as dimensões das matrizes envolvidas.

As equações das estacas, consideradas aqui como elementos de barras dispostas no meio contínuo, são analisadas pelo método dos elementos finitos. Estas equações, combinadas com as obtidas do método dos elementos de contorno, resultam em um sistema único de equações, a partir do qual são determinados coefícientes que representam a rigidez do conjunto solo-fundação. Este procedimento possibilita analisar a superestrutura considerando-se o comportamento global do conjunto solo-fundaçãosuperestrutura.

Uma extensão a esta formulação foi desenvolvida para considerar a resistência de ponta por meio da inclusão de um nó interno adicional ao elemento de extremidade da estaca. Tal consideração, permite análises mais abrangentes e tornam o modelo mais consistente, apresentando resultados, bastante satisfatórios, quando comparados a outras formulações existentes.

Visando o estudo mais adequado da fundação, foi proposto um modelo para a análise da plastificação do solo nos pontos de interface com as estacas.

Algumas simulações foram feitas com a utilização desse modelo para análise do comportamento não-linear do solo, mostrando a viabilidade da formulação proposta. 
Embora seja um modelo que considera o solo como um material homogêneo é possivel vislumbrar uma análise mais realista com o aprimoramento dessa proposta.

Mesmo com todas as dificuldades de caracterização do solo, os resultados obtidos para exemplos disponiveis na literatura, mostraram-se muito próximos das cargas limites apresentadas nos ensaios.

Verificou-se também que, para solos com módulo de deformação elevado, os deslocamentos são mais próximos dos resultados dos ensaios.

A partir desses resultados iniciais é possível acrescentar à formulação desenvolvida outros fatores que influenciam nesse tipo de análise. Para tanto, podem ser implementados outros critérios de escoamento ou curvas tensão-deformação para o solo, sem grandes dificuldades.

Pode ainda ser considerada a variação das propriedades dos solo, através do emprego de sub-regiões e a inclusão no modelo de uma formulação que considere o escorregamento das estacas. 


\section{REFERÊNCIAS BIBLIOGRÁFICAS}

ABEL, N.H. (1881) Ouvres complètes. Norvegien Christiania, v.1.

ABRAMOWITZ, M.; STEGUN, I.A. (1972) Handbook of mathematical functions

New York, Dover Publications.

ALARCON, E.; SUAREZ, C.G.; REVERTER, A. (1983) Effective and neutral estress in soil using boundary element methods. In: BREBBIA, C.A. et al. eds. Boundary Element V. Berlin: Springer-Verlag. p.655-663.

ANDERSSEN, R.S. et al. (1980) The application and numerical solution of integral equations. Alphen aan den Rijn, The Netherlands: Sijthoff and Noordhoff, 1980.

ARGYRIS, J.H; KELSEY, S. (1960) Energy theorems and structural analysis. London: Butterworths.

BANERJEE, P.K. (1976) Integral equations methods for analysis of piece-wise non-homogeneous three-dimensional elastic solids of arbitrary shape. Int. Joumal Mechanics Science, v. 18, p.293-303.

BANERJEE, P.K; BUTTERFIELD, R. (1976) An integral equation method for analysis of boundary value problems in irregularly stratified media. In: GUDEHUS, G. ed. Num. Methods Soil Rock Mech. p. 141-163.

BANERJEE, P.K.; DRISCOLL, R.M. (1976) Three-dimensional analysis of raked pile groups. Proc. Inst. Civ. Eng., n.61, p.653-671.

BANERJEE, P.K. (1978) Analysis of axially and laterally loaded pile groups. In: SCOTT, C.R., ed. Developments in soil mechanics - 1. Barking: Appl. Sci. Publ., p.317-346.

BANERJEE, P.K.; DAVIES, T.G. (1984) Advanced implementation of boundary element methods for three-dimensional problems of elastoplasticity and viscoplasticity. In: MUKHERJEE, S., BANERJEE, P.K., eds. Developments in boundary element methods -3 . Applied Science Publ. 
BANERJEE, P.K.; WILSON, R.B. (1985) Development of a large B.E.M. system for three-dimensional inelastic analysis. In: CRUSE, T.A. et al., eds. Advanced topics in boundary element analysis, p. 1-20. New York, ASME.

BARBIRATO, J.C.C. (1991) Formulação do método dos elementos de contorno para sólidos elásticos tridimensionais, baseada na solução fundamental de Mindlin. São Carlos. Dissertação (Mestrado) - Escola de Engenharia de São Carlos, Universidade de São Paulo.

BARRETO, S.F.A. (1995) Análise elastodinâmica de placas através do método dos elementos de contorno interação solo-estrutura. São Carlos. Tese (Doutorado) - Escola de Engenharia de São Carlos, Universidade de São Paulo.

BEER, G.; MEEK, J.L. (1981) Coupled finite element-boundary element analysis of finite domain problems in geomechanics. In: HINTON, E., BETTESS, P, LEWIS, R.W., ed. Numerical methods for coupled problems. Swansea: Pineridge Press, p.605-629.

BEER, G. (1983) Finite element boundary element and coupled analysis of unbounded problems in elastostatics. Int. J. Num. Meth. Ing., v.19, p.567-580.

BEER, G. (1986) Implementation of combined boundary element-finite element analysis with applications in geomechanics. In: BENERJEE, D.K., WATSON, J.O., eds. Developments in boundary element methods - 4. Barking: App. Sci. Publ., p.191-225.

BEER, G.; SWOBODA, G. (1988) Application of advanced boundary element and coupled methods in geomechanics. In: CRUSE, T.A., ed. Advanced Boundary Element Methods. Berlin: Springer-Verlag, p. 19-28.

BEER, G. (1989) Application of 3-D boundary element and coupled analysis in geomechanics: case studies. In: SWOBODA, G. ed. Num。 Methods Geomechanics. Rotterdam: Balkema, v.4, p. 2209-2216.

BELYTSCHKO, T.; LU, Y.Y. (1991) Singular integration in variationally coupled FEBE method. Journal Lng. Mech. - ASCF. v.117(4), p.820-835.

BESKOS, D.E. (1988) Boundary element methods in geomechanics. In: BREBBIA, C.A. ed. Boundary Llement $X$. Berlin: Springer-Verlag. v. 4, p.3-28. 
BESKOS, D.E. (1988) Boundary element methods in geomechanics. In: BREBBIA, C.A. ed. Boundary Element X. Berlin: Springer-Verlag. v. 4, p.3-28.

BETTI, E. (1872) Teoria dell elasticita. Il Nuovo Ciemento, v.7-10.

BÉZINE, G. (1978) Boundary integral formulation for plate flexure with arbitrary boundary conditions. Mech. Res. Comm., v.5, n.4, p.197-206.

BÉZINE, G. (1980) A mixed boundary integral: finite element approach to plate vibration problems. Mech. Res. Comm., v.7, n.3, p.141-150.

BÉZINE, G.; BONNEAU, D. (1981) Integral equation method for the study of two dimensional stokes flow. Acta Mechanica, v.41, p.197-209.

BÉZINE, G; CIMETIERE, A.; GELBERT, J.P. (1985) Unilateral buckling of thin plates by the boundary integral equation method. Int. J. Num. Meth. Eng., v.21, p.2189-2199.

BOOKER, J.R. et al. (1989) Some recent applications of numerical methods to geotechnical analysis. Computers \& Structures, v.31, n. 1, p.81-92.

BOUSSINESQ, J. (1885) Applications des potentiels à l'études de l'équilibre et du mouviment des solides élastiques. Paris: Gauthier-Villars.

BRADY, B.H.G.; BRAY, J.W. (1978) The boundary element method for determining stresses and displacements around long opening in a triaxial stress field. Int. Rock Mech. Mining Sci., v. 15, p.21-28.

BRADY, B.H.G. (1979) A direct formulation of the boundary element method of stress analysis for complete plane strain. Int. J. Roch Mech. Min. Sci, v.16, p. $225-244$.

BRADY, B.H.G.; WASSYNG, A. (1981) A coupled finite element-boundary element method of stress analysis. Int. I. Rock Mech. Mining Sci., v.18, p.475-485.

BREBBIA, C.A. (1978a) Weighted residual classification of approximate methods. Applied Mathematical Modelling, v.2, n.3.

BREBBIA, C.A. (1978b) The boundary element method for engineers. London: Pentech Press. 
BREBBIA, C.A. (1978c) ed. Recent advances in boundary element methods: Int. Conf. Boundary Element Methods, 1st., Southampton. London; Pentech Press. Preceedings.

BREBBIA, C.A; GEORGIOU, P. (1979) Combination of boundary and finite elements in elastostatic. Appl. Math. Modelling, v.3, p.212-220.

BREBBIA, C.A. (1980) ed. New developments in boundary element methods: Int. Conf. Boundary Element Methods, 2nd, Southampton. Southampton: CML. Proceedings.

BREBBIA, C.A. (1981) ed. Boundary element methods: Int. Conf. Boundary Element Methods, 3rd, California, 1981. Berlin: Springer-Verlag. Proceedings.

BREBBIA, C.A. (1982) ed. Boundary element methods in engineering: Int. Conf. Boundary Element Methods, Southampton, 4th. Berlin: Springer-Verlag. Proceedings.

BREBBIA, C.A.; TELLES, J.C.F; WROBEL, L.C. (1984) Boundary element techniques. Berlin: Springer-Verlag.

BREBBIA, C.A.; DOMINGUEZ, J. (1984) Boundary elements: an introductory course. Southampton: CML, Publ.

BRUNET, M. (1982) Numerical analysis of viscoplasticity using the boundary element method. In: BREBBIA, C.A., eds. Boundary Element Method Cng. Berlin: Springer-Verlag, p.349-362.

BUDHU, M; DAVIES, T.G. (1988) Analysis of laterally loaded piles in sof clays. J. Geotch. Eng. - ASCE. v.114(1), p.21-39.

BUI, H.D. (1978) Some remarks about the formulation of three-dimensional thermoelastoplastic problems by integral equations. Int. J. Solid Structures, v. 14, p. $935-939$

BUTTERFIELD, D.R; BANERJEE, P.K. (1970) A note on the problem of a pile reinforced half space. Géotechnique, v.20, n.1, p.100-103.

BUTTERFIELD, R; BANERJEE, P.K. (1971a) The elastic analysis of compressible piles and piles group. Géotechnique, v.21, n. 1, p.43-60. 
BUTTERFIELD, R; BANERJEE, P.K.(1971b) The problem of pile group-pile cap interaction. Géotechnique, v.21, n.2, p.135-142.

BUTTERFIELD, R.; PAIVA, J. (1993) Stresses in groups of axially loaded embedded fixing of differing length. Boundary Element Techniques and Singularity Methods in Engineering. Poland: Wroclaw. p.205-

CAI, R.Y.; ZENG, Z.J.; FEN, C. (1991) Partitioning technique for boundary element method. Computers and Structures, v. 36, n. 5-6, p. 537-545.

CERRUTI, V. (1882) Mem. fis. mat., Acc. Lincei, Roma.

CATHIE, D.N.; BANERJEE, P.K. (1982) Boundary element methods for plasticity and creep including visco-plastic approach. Res. Mechanics, v.4, p.3-22.

CHANDRA, A; MUKHERJEE, S. (1984) Boundary element formulations for large strain-large deformation problems of viscoplasticity. Int. J. Solids Structures, v. 20, p. 41.53 .

CHANDRA, A; MUKHERJEE, S. (1985) A boundary element formulation for sheet metal forming. Appl. Math. Modelling, v.9, p.175-182.

CHEN, G.R; QIAN, J. (1986) The coupling of BEM and FEM used in stress analysis of the structures on the half infinit foundations. In: DU, Q.H., eds. Boundary element VIII. Oxford: Pergamon Press, p. 689-696.

CHEN, H.S.; MEI, C.C. (1974) Oscillations and wave forces in a man-made harbour. In: NAVAL HIDRO. SYMP., 10th, Cambridge, USA. Proceedings.

CHEN, W.F. (1982) Plasticity in reinforced concrete. USA, McGraw-Hill, Inc.

CHEUNG, Y.K.; LEE, P.K.K.; ZHAO, W.B. (1991) Elastoplastic analysis of soil-pile interaction. Computers and Geotechnics, v.12, p. 115-132.

CHIN, J.T; CHOW, Y.K. (1990) Numerical analysis of axially loaded vertical pile and pile groups. Comp. Geotech. v.9(4), p.273-290.

CHIN, J.T.; CHOW, Y.K.; POULOS, H.G. (1993) Numerical analysis of axially loaded vertical piles and pile groups. Computer and Geotechnics. v.9, n.4, p.273290. 
CHOW, Y.K. (1986) Analysis of vertically loaded pile groups. Int. J. Anal. Meth. Geomech. v. 10, p.59-72.

CODA, H.B. (1993) Análise tridimensional transiente de estruturas pela combinação entre o método dos elementos de contorno e o método dos elementos finitos. São Carlos. Tese (Doutorado) - Escola de Engenharia de São Carlos, Universidade de São Paulo.

COOK, R.D.; MALKUS, D.S.; PLESHA, M.E. (1989) Concepts and applications of finite element analysis. 3.ed. New York, John Wiley \& Sons.

COSTA JR., J.A.; BREBBIA, C.A. (1985) Elastic buckling of plates using the boundary element method. In: BREBBIA, C.A., MAIER, G., eds. Boundary element VII. Berlin: Springer-Verlag.

CROTTY, J.M. (1982) A block equation solver for large unsymmetric matrices arising in the boundary integral equation method. Int. J. Num. Meth. Eng., v. 18, p. 997 1017.

CRUSE, T.A. (1969) Numerical solutions in three dimensional elastostatics. Int. Journal of Solid and Structures, v.5, p.1259-1274.

CRUSE, T.A.; VANBUREN, W. (1971) Three dimensional elastic stress analysis of a fracture specimen with an edge crack. Int. Jounnal Mech., v.7, p.1-15.

CRUSE, T.A. (1973) Application of the boundary-integral equation method to three dimensional stress analysis. Computer and Structures, v.3, p.509-527.

CRUSE, T.A. (1974) An improved boundary-integral equation method for three dimensional elastic stress analysis. Computer and Structures, v.4, p.741-754.

DIVAKAR, M.P.; FAFITIS, A. (1989) Fortran77 program for boundary element equations. ASME Review, v.11, n.3, p.45-56.

DOMINGUEZ, J.; ALARCON, E. (1981) Elastodynamics. In: BREBBIA, C.A., ed. Progress in boundary element methods. London, Pentech Press, v.1.

DRUCKER, D.C.; PRAGER, W. (1952) Soil mechanics and plastic analysis or limit design. Quart. Appl. Math., v. 10, p.157.165. 
EBERHARDSTEINER, J.; MANG, H.A; TORZICKY, P. (1993) Hybrid BE-FE stress analysis of the excavation of a tunnel bifurcation on the basis of a substructuring technique. In: KANE, J. H. et al. eds. Adv. Boundary Elem. Tech., p. 105-128.

EL REFAEE, M.M; WU, J.C; LEKOUDIS, S.G. (1982) Solution of the compressible navier-stokes equations using the integral methods. AlAA Journal, v.20, p.356-362.

FERREIRA, W.G. (1990) Implementação de elemento de colocặão não nodal para a análise tridimensional pelo método dos elemntos de contomo. Rio de Janeiro. Dissertação (Mestrado) - COPPE, Universidade Federal do Rio de Janeiro.

FERRO, N.C.P.; VENTURINI, W.S. (1991) Formulação de um elemento de fundação através do método dos elementos de contorno. In: JORNADAS SUL-AMERICANAS DE ENGENHARIA ESTRUTURAL, 25., Porto Alegre, 1991. Anais. v. 1, p. $421-430$.

FERRO, N.C.P.; VENTURINI, W.S. (1992) BEM-FEM coupling for building structure analysis. In: INTERNATIONAL CONFERENCE ON BOUNDARY ELEMENT METHODS, 14th, November, Seville, 1992. Proceedings. v.2, p. $451-465$.

FERRO, N.C.P. (1993) Uma combinação MLC/MET para análise de fundaçóes enrijecidas por estacas. São Carlos. Tese (Mestrado) - Escola de Engenharia de São Carlos, Universidade de São Paulo.

FERRO, N.C.P; VENTURINI, W.S. (1995) Estudo do efeito de segunda ordem em estruturas de edifícios considerando-se a ligação elástica com o semi-infinito. In: JORNADAS SUDAMERICANAS DE ENGENIERIA ESTRUTURAL, 27. Tucumán, 1995. Anais. San Miguel de Tucumán, 1995, v.5, p.175-86.

FLOREN, H.; DUDDECK, H. (1993) Efficient coupling of BEM and FEM in geomechanics. In: BREBBIA, C.A, RENCIS, J.J. eds. Poundary element XV. Southampton: Computational Mechanics Publications. p. 527-537. 
FREDHOLM, I. (1903) Sur une classe d'equation fonctionelles. Acta Math. v.27, p. 365-390.

GERE, J.M; WEAVER JR., W. (1981) Análise de estruturas reticuladas. Rio de Janeiro: Guanabara Dois.

GIL RODRIGUEZ, J.C. (1986) Sobre o emprego de método dos elementos de contorno em problemas elásticos bidimensionais. São Carlos. Dissertação (Mestrado) - Escola de Engenharia de São Carlos, Universidade de São Paulo.

GIODA, G.; CARINI, A; CIVIDINI, A. (1984) A visco-elastic stress analysis of tunnels. In: BREBBIA, C.A., ed. Boundary elements VI. Berlin: Springer-Verlag.

HAMMER, P.C; MARLOWE, O.J; ATROUD, A.H. (1956) Numerical integration over simplex and cones. Math. Tables and Other Aids to Computation, v. 10, p. 130-137.

HAUNG, Q; CRUSE, T.A. (1993) Some notes on singular integral techniques in boundary element analysis. Int. J. Num. Meth. Eng., v.36,n.15, p.2643-2659.

HESS, J.L.; SMITH, A.M.O. (1967) Calculation of potential flow about arbitrary bodies. In: KTCHEMANN, D., ed. Progress in aeronautical sciences - 8 . London: Pergamon, p.1-138.

HESS, J.L. (1975) Review of integral-equation technique for solving potential-flow problems with emphasis on the surface-source method. Computer Methods in Applied Mechanics and Engineering, v. 5, p. 145-196.

HILL, R. (1947) The mathematical theory of plasticity. Oxford: Oxford University Press.

HODGE, P.G. (1959) Plastic analysis of structures. McGraw-Hill.

JASWON, M.A. (1963) Integral equation methods in potential theory 1. Proc. Royal Society Ser., v.A275, p.23- 32 .

KAMYIA, N.; SAWAKY, Y. (1982) An integral equation approach to finite deflection of elastic plates. Int. Jo Non-Linear Mechanics, v. 17, n.3, p.187-194. 
KANE, J.H; KUMAR, B.L.K.; SAIGAL, S. (1990) Arbitrary condensing, noncondensing solution strategy of large scale, multi-zone boundary element analysis. Computer Methods in Applied Mechanics and Engineering, v. 79, n. 2, p.219-244.

KANE, J.H.; GURU, P.K. (1993) Sparse blocked equation solving techniques in boundary element analysis. In: KANE, J. K. et al. Adv. Boundary Elem. Tech. Berlin: Springer-Verlag, p.209-238.

KAYNIA, A.M. (1993) Piles. In: MANOLIS, G.D; DAVIES, T.G., eds. Comp. Meth. Contact Mech. Southampton: Computational Mechanics Publications. p.209-242.

KELLY, D.W.; MUSTOE, G.G.W.; ZIENKIEWICZ, O.C. (1979) Coupling boundary element methods with other numerical methods. In: BANERJEE, P. K., BUTTERFIELD, R., ed. Developments in boundary element methods - 1 . Barking: Appl. Sci. Publ.

KOMATSU, J.S. (1995) Estudo de problemas de escavação através da combinação elemento de contorno e elementos finitos. São Carlos. Tese (Doutorado) - Escola de Engenharia de São Carlos, Universidade de São Paulo.

KUPRADZE, V. D. (1965) Potential methods in the theory of elasticity. Jerusalem: Israel Program for Scientific Translations.

LACHAT, J.C. (1975) A further development of the boundary integral technique for elastostatics. Southampton. Ph. D. Thesis - University of Southampton.

LEE, C.Y. (1993) Pile group settlement analysis by hydrid layer approach. Jourmal of Geotechnical Rngineering. v. 119, n.6, p.984-997.

LEUNG, C.F.; CHOW, Y.K. (1985) Analysis of laterally loaded pile groups. In: ADEY, R.A. Lng. Software IV. Berlin: Springer-Verlag. v.3, p. 51-59

LIOUVILLE, V. (1837) Sur le développment des fonctions. Journal de Mathémetiques Pures et Appliqués, v.2, p. 16-35.

LODE, W. (1926) Versuche ueber den einfluss der mitt leren hauptspannung auf das fliessen der metalle eisen kupfer und nickel. Zeitschrift fuer Physik. v.36, p.913939. 
LU, Y.Y.; BELYTSCHKO, T.; LUI, W.K. (1991) A variationally coupled FE-BE method for elasticity and fracture mechanics. Computer Methods in Applied Mechanics and Engineering, v.85(1), p.21-37.

LUTZ, E. (1992) Exact Gaussian quadrature methods for near-singular integrals in the boundary element method. Engineering Analysis with Boundary Elements, v.9, n. 3, p. 233-245.

MADHAV, M.R; BUDKOWSKA, B.B. (1986) Analysis of efficiency of axially loaded pile groups. In: INT. SYMP. NUM. MODELS GEOMECH., 2nd. Proceedings. Ghent: Jackson Publ. p.505-510.

MAIER, G.; NOVATI, G. (1983) Elasto-plastic boundary element analysis as a linear complementary problem. Appl. Math. Modelling, v.7, p.74m82.

MAIER, G.; NOVATI, G.; PARREIRA, P. (1984) On boundary element elastic and inelastic analysis in the presence of cyclic symmetry. In: BREBBIA, C.A., ed. Boundary elements VI. Berlin: Springer-Verlag.

MAIER, G.; NOVATI, G.; (1987) Boundary element elastic analysis of layered soils by a successive stiffness method. International Journal for Numerical and Analytical Methods in Geomechanics, v. 11, n. 5, p. 435-447.

MAIER, G.; NOVATI, G.; (1988) Elastic analysis of layered soils by boundary elements: comparative remakes of various approaches. In: SWOBODA, G. ed. Num. Meth. Geomech. Rotterdam: Balkema. p. 925-933.

MAIER, G; NOVATI, G; OHGA, M; SHIGEMATSU, T; HARA, T. (1993a) Boundary element transfer-matrix method for plated structures discussion. I. Thing. Mech. - ASCE, v. 119(2), p. 412-414.

MANOLIS, G.D.; BESKOS, D.E. (1981) Dynamic stress concentration studies by boundary integrals an Laplace transform. Int. J. Num. Meth. Lng., v.17, p. $573-599$.

MANOLIS, G.D; DAVIES, T.G; BESKOS, D.E. (1993) Overview of boundary element techniques in geomechanics. In: MANOLIS, G.D. \& DAVIES, T.G. eds. Boundary Elem. Tech. Geomech. Southampton: Computational Mechanics Publications. p.1-36. 
MANSUR, W.J. (1983) Time stepping scheme to solve transiente wave propagation problems using the boundary element metod. Southampton. Ph.D. Thesis - University of Southampton.

MASSONET, C.E. (1965) Numerical use for integral procedures. In: Zienkiewicz, O. C.; Hollister G. S., eds. Stress analysis. London: Wiley, cap. 10.

MATTES, N.S.; POULOS, H.G. (1969) Settlement of single compressible pile. Journal of the Soil Mechanics and Foundations Division, ASCE, v.97, n.SM1, p. 189- 207.

McDONALD, B.H.; WEXLER, A. (1972) Finite element solution of unbounded field problems. IEE Trans. Microwave Theory and Technique, MTT-20, p.841-847.

MEEK, J.L.(1971) Matrix structural analysis. New York: McGraw-Hill.

MEEK, J. L. (1988) BECOUP - A program for coupled boundary and finite element analysis in 3-D elastostatics. BREBBIA, C,A, ed. Boundary elements $\mathbb{X}$; stress analysis. Southampton: CML Publ, v.3, p.639-655.

MENDELSON, A. (1968) Plasticity: theory and aplication. Robert E. Krieger Publ. Co.

MESSAFER, T.; COATES, L.E. (1989) An application of FEM/BEM coupling to foundation analysis. In: BREBBIA, C. A; CONNOR, J. J., eds. Advances in boundary elements -3 : stress analysis. Southampton, CML, Publ, p.211-223.

MELOSH, R.S. (1961) A stiffness matrix for the analysis of thin plates in bending. Journal Aeronaut. Science, v.28, n.1, p.34-42.

MIKHLIN, S.G. (1957) Integral equations. London; Pergamon Press.

MIKHLIN, S.G. (1965a) Multi-dimensional singular integrals and integral equations. Oxford: Pergamon Press.

MIKHLIN, S.G. (1965b) Approximate methods for solution of differential and integral equations. Oxford: Pergamon Press.

MINDLIN, R.D. (1936) Force at a point in the interior of a semi-infinite solid. J. Physies, v, 7, p. 195-202. 
MISES, R.Von. (1913) Mechanik der festen koerper in plastisch deformablen zustand.

Math. Phys., Goettinger Nachr., v.k1, n.582-592.

MITA, A,; LUCO, J.E. (1986) Response of embedded foundations: a hybrid approach. In: ASCE EM SPEC. CONF. DYN. RESP. STR., 3rd. Los Angeles, 1986. Proceedings. p. 708-715.

MORJARIA, M; MUKHERJEE, S. (1980) Inelastic analysis of transverse deflection of plates by the boundary element method. J. Appl. Mechanics, ASME, v.47, n.2, p.291-196.

MUSTOE, G.G. (1984) Advanced integration schemes for boundary elements and volume cells for two and three-dimensional nonlinear analysis. In: BANERJEE, P.K., MUKHERJEE, S., eds. Developments in boundary element methods $\approx 3$. Barking: Applied Science Publ.

MUKHERJEE, S. (1982) Boundary element method in creep and fracture. Barking, U.K.: Elsevier Applied Science.

MUKHERJEE, S.; CHANDRA, A. (1984) Boundary element formulations for large-strain large deformation problem of plasticity and viscoplasticity. In: BANERJEE, P.K., MUKHERJEE, S., eds. Developments in boundary element methorls -3 . Barking: Apll. Sci. Publ.

MUKHERJEE, S; RAJIYAH, H. (1987) Analysis of metal forming problems by the boundary element method. In: BREBBIA, C.A., VENTURINI, W.S., eds. Boundary element techniques: applications in stress analysis and heat transfer. Southampton: CML.

MUSKHELISHVILI, N.I. (1953) Some basic problems of mathematical theory of elasticity. Groningen, Holand: Noordhoff.

NAKAGUMA, R.K. (1979) Threedimensional elastostatic using the boundary element method. Southampton. $\mathrm{Ph}$. D. Thesis - University of Southampton.

NAYAK, G.C.; ZIENKIEWICZ, O.C. (1972) Convenient form of stress invariants for plasticity. Joumal of Structure Div. Proc. ASCL, p.949-954. 
NOVATI, G.; BREBBIA, C.A. (1982) Boundary element formulation for geometrically nonlinear elastostatics. Appl. Math. Modelling, v.6, p.136-138.

OHKAMI, T.; KUSAMA, T. (1985) Coupled boundary element/finite element analysis in geomechanics including body forces. Comp. Geotech. v.1, p. 263-278.

OSIAS, J.R.; WILSON, R.B.; SEITELMAN, L.A. (1977) Combined boundary integral equation finite element analysis of solids. In: SYMPOSIUM ON INNOVATIVE NUMERICAL ANALYSIS IN APPLIED ENGINEERING SCIENCE; 1st, Versailles, CETIM, 1977. Proceedings.

OWEN, D.R.J.; HINTON, E. (1986) Finite elements in plasticity: theory and practice. U.K., Pineridge Press limited.

PAIVA,J.B.; BUTTERFIELD, R. (1994) Numerical analysis of plate-soil interaction. In: PAPADRAKAKIS, M; TOPPING, G.H.V., eds. Advances in computational mechanics. pp. $275-281$.

PAK, R.Y.S.; JI, F. (1993) Rational mechanics of axial soil-pile interaction, Joumal of Engineering Mechanics. v. 119 , n. 4, p.813-832.

PAULA, F.A.; TELLES, J.C.F.; MANSUR, W.J. (1987) Combination of boundary elements and finite elements to solve two-dimensional elasticity problems. In: BREBBIA, C.A., VENTURINI, W.S., eds. Poundary element techniques: applications in stress analysis and heat transfer. Southampton: CML Publ, p. $163-176$.

POULOS, H.G; DAVIS, E.H. (1968) The settlement behaviour of single axially loaded incompressible piles and piers. Geotechnique, v. 18, p.351-371.

POULOS, H.G. (1968) Analysis of the settlement of pile groups. Geotechnique, v.18, p. $449-471$.

POULOS, H.G. (1971a) Behavior of laterally loaded piles: I- single piles, Journal of the Soil Mechanies and Foundations Division, ASCE, v.97, n.SM5, p.711-731.

POULOS, H.G. (1971b) Behaviour of laterally loaded piles: II- piles groups, Journal of the Soil Mechanies and Foundations Division, ASCE, v.97, n.SM5, p. $733-751$. 
POULOS, H.G.; DAVIS, E.H. (1980) Pile foundation analysis and design. New York: John Wiley \& Sons.

POULOS, H.G. (1982) Developments in the analysis of static and cyclic lateral response of piles. In: EDMONTON. Num. Meth, Geomech. Balkema, p.11171135.

POULOS, H.G. (1989) Pile behaviour - theory and application. Geotechnique. v.39, n. 3, p. $365-415$.

PROENÇA, S.P.B. (1989) Notas sobre análise não-linear física de estruturas; parte I: teoria da plasticidade e técnicas numéricas. São Carlos, EESC.

RAMALHO, M.A. (1990) Sistema para análise de estruturas considerando interação com o meio elástico. São Carlos, 1990. Tese (Doutorado) - Escola de Engenharia de São Carlos-USP.

RAMALHO, M.A, VENTURINI, W.S. (1990) Formulação de um elemento para a discretização de sapatas rígidas com base no método dos elementos de contorno. In: CONGRESSO IBERO LATINO AMERICANO SOBRE MÉTODOS COMPUTACIONAIS PARA ENGENHARIA, 11., Rio de Janeiro, 1990. Anais. v.2, p.903-912.

REZAYAT, M. (1992) Fast decomposition of matrices generated by the boundary element method. International Journal for Numerical Methods in Lingineering, v. 33, n.6, p.1109-1118.

RICARDELLA, P.C. (1973) An implementation of the boundary integral technique for planar problems in elasticity and elastoplasticity. Pittsburg: Dept. Mech. Engng., Carnegie-Mellon University. (Report n.SM-73-10)

RIZZO, F.J. (1967) An integral equation approach to boundary value problems of classic elastostatic. Quarterly of Applied Mathematics. v.25, n.1, p.83-95.

SÁ, P.A.C.O., TELLES, J.C.F. (1986) Análise de problemas de elasticidade linear tridimensional pelo método dos elementos de contorno utilizando as soluções fundamentais de Kelvin e Mindlin. In: CONGRESSO LATINO-AMERICANO 
SOBRE MÉTODOS COMPUTACIONAIS PARA ENGENHARIA, 7., São Carlos. Anais. v. 1, p. $43-60$.

SHARMA, K.G. et al. (1985) Condensation of boundary element stiffiness matrix in FEBEM analysis. Comm. Appl. Num. Meth., v.1, p.61-65.

SHAW, R.P.; FALBY, W. (1977) A combined finite element-boundary integral equation method. In: SYMPOSIUM ON INNOVATIVE NUMERICAL ANALYSIS IN APPLIED ENGINEERING SCIENCE, 1st, Versailles, CETIM. Proceedings.

SILVA, J.J.R. (1989) MEC3D - Um programa para análise elástica tridimensional com o método dos elementos de contorno. Rio de Janeiro. Dissertação (Mestrado) - COPPE, Universidade Federal do Rio de Janeiro.

SOMIGLIANA, C. (1886) Sopra l'equilibrio di un corpo elastico isotropo. II Nuovo Ciemento, v.17-19.

SOUTHWELL, R.V. (1946) Relaxation methods in theoretical physics. London: Oxford University Press. (The Oxford Engineering Science Series)

SWEDLON, J.L; CRUSE, T.A. (1971) Formulation of boundary integral equations for the three dimensional elastoplastic flow. Int. Journal Solids Structures, v.7, p. $1673-1683$.

SYMM, G.T. (1963) Integral equation methods in potential theory II. Proc. Royal Society Ser., v.A275, p.33- 46.

TANAKA, M. (1984) Large deflection analysis of thin elastic plates. In: BANERJEE, P.K., MUKERJEE, S., eds. Developments in boundary element methods - 3 . London: Elsevier.

TEJERINA CALDERÓN, E. (1996) Sobre o uso do método dos elementos de contorno - MEC para o estudo da interação de placas com o meio contínuo. São Carlos. Tese (Doutorado) - Escola de Engenharia de São Carlos Universidade de São Paulo.

TELLES, J.C.F., BREBBIA, C.A. (1979) The application of the boundary element method to plasticity. Appl. Math. Modelling, v.3, p.466-470. 
TELLES, J.C.F., BREBBIA, C.A. (1980a) The boundary element method in plasticity. In: BREBBIA, C.A., ed. New development in boundary element methods. Southampton: CML, p. 295-317.

TELLES, J.C.F., BREBBIA, C.A. (1980b) Elastoplastic boundary element analysis. In: WUNDERLIUCH, W. et al, eds. Proc. Europe-U.S. workshop on nonlinear finite element analysis in structural mechanics 1980. Berlin: Springer-Verlag, p.403-434.

TELLES, J.C.F. (1986) Implementation of triangular elements into the Beasy System. Internal report, Computational Mechanics. Southampton. UK.

TELLES, J.C.F. (1987) A self-adaptive co-ordinate transformation for efficient numerical evaluation of general boundary element integrals. International Journal for Numerical Methods in Engineering, v.24, p.959-973.

TELLES, J.C.F.; OLIVEIRA, R.F. (1994) Third degree polynomial transformation for boundary element integrals: Further improvements. Engineering Analysis with Boundary Clements, v. 13, p. 135-141.

TIMOSHENKO, S; GOODIER, J.N. (1975) Teoria de la clasticidade. 2. ed. Bilbao, Urmo, S. A de Ediciones.

TOTTENHAN, H. (1979) The boundary element method for plates and shells. In: BANERJEE, P.K., BUTTERFIELD, R., eds. Developments in boundary element methods. Barking: Appl. Sci. Publ.

TURNER, MJ. et al. (1956) Stiffness and deflection analysis of complex structures. Journal Aeronaut. Science, v.23, n.9, p.805-823.

VALLABHAN， C.V.G; SIVAKUMAR， J.; RADHAKRISHNAN， N. (1984) Application of boundary element method for soil-structure interaction problems. In: INTERNATIONAL CONFERENCE ON BOUNDARY ELEMENT METHODS, 6th, 1984. Proceedings. p.27-39.

VALLABHAN, C.V.G., SIVAKUMAR, J. (1986) Coupling of BEM and FEM for 3D problems in geotechnical engineering. In: BREBBIA, C.A., CONNOR, J.J, eds. BETECH 86. SOUTHAMPTON: CML PUBL,, p.675-686. 
VALLABHAN, C.V.G. (1987) Coupling of BEM/FEM technology: an overview. In: BREBBIA, C.A., VENTURINI, W.S., eds. Boundary element techniques: applications in stress analysis and heat transfer. Southampton: CML Publ, p. $15-30$.

VAN BUREN, W. (1968) Res. Ept. 68-1D7-MEKMA-R2, Westinghouse Research Laboratories, Pittsburgh.

VENTURINI, W.S. (1982) Application of the boundary element formulation to solve geomechanical problems. Southampton. Ph.D. Thesis - University of Southampton.

VENTURINI, W.S.; BREBBIA, C.A. (1983) Some applications of the boundary element methods in geomechanics. Int. Journal Num. Anal. Meth. Geomechanics, v.7, p.419 433 .

VENTURINI, W.S.; BREBBIA, C.A. (1984) Boundary element formulation for nonlinear applications in geomechanics. Appl. Math. Modelling, v.8, p. 251-260.

VENTURINI, W.S. (1988) Um estudo sobre o método dos elementos de contorno e suas aplicações em problemas de engenharia. São Carlos. Tese (Livre-docência) - Escola de Engenharia de São Carlos-USP.

VENTURINI, W.S. (1994) A new boundary element formulation for crack analysis. In; BREBBIA, C.A. ed., Boundary Element Method XVI, pp. 405412. Southampton, UK.

VOLTERRA, V. (1956) Opere mathematiche. Acad. Naz Lincei, Rome, v.2, p. $216-275$.

VON ESTORFF, O.; KAUSEL, E. (1989) Coupling of boundary and finite elements for soil-structure interaction problems. Carthquake Engineering \& Structural Dynamics. v. 18, n. 7, p. $1065-1075$.

WATSON, J. O. (1968) Report n $^{0}$ CE/18/1968. Southampton University.

WATSON, J. O. (1972) Varational methods in engineering . In: INTERNATIONAL CONFERENCE, v. 2, Southampton. 
WATSON, J. O. (1973) The analysis of thick shells with holes, by integral representation of displacement. Southampton, 1973. $\mathrm{Ph}$. D. Thesis - University of Southampton.

WILSON, E.L. (1974) The static condensation algorithm. Int. J. Numerical Methods in Engineering, v.8, p.198-203.

WOLF, J.P. (1985) Dynamic soil-structure interaction. Prentice Hall.

WOLF, J.P.; DARBRE, G.R. (1986) Nonlinear soil-structure interaction analysis based on the boundary-element method in time domain with application to embedded foundation. Carthq. Eng. Struct. Dyn.v.14(1), p.83-101.

WU, J.C. (1980) Integral-representation approach for the numerical solution of turbulent flow problems. AIAA Jourmal, v.16, p.948-955.

WU, J.C. (1982) Problems of general viscous flow. In: BANERJEE, P.K., SHAW, R.P., eds. Development in boundary element method - 2. Barking: Appl. Sci. Publ.

WU, J.C. (1985) Boundary element methods and in homogeneous elliptic differential equation. In: BREBBIA, C.A., MAIER, G., eds. Poundary elements VII. Berlin: Springer-Verlag.

WU, J.C. (1987) Boundary element solution of viscous flow problems. In: BREBBIA, C.A., VENTURINI, W.S., eds. Poundary element techniques: application in fluid flow and computational aspects. Southampton: CML.

YANO, H.; KIEDA, A. (1980) An approximate method for solving two-dimensional low-Reynolds-number flow past arbitrary cylindrical bodies. J. Fluid Mech., v.97, p. $157-179$.

ZIENKIEWICZ, O.C; VALLIAPPAN, S.; KING, I.P. (1969) Elasto-plastic solutions of engineering problems 'initial stress', finite element approach. Int. Journal for Numerical Methods in Engineering, v.1, p.75-100.

ZIENKIEWICZ, O.C (1971) The finite element method in engineering science. 2. ed. London, McGraw-Hill. 
ZIENKIEWICZ, O.C.; KELLY, D.W.; BETTESS, P. (1977) The coupling of the finite element method and boundary solution procedures. Int. Journal for Numerical Methods in Engineering, v. 11, p.355-375. 